\title{
The effects of methyl-donor deficiency on mutation induction and transgenerational instability in mice
}

A thesis submitted for the degree of

Doctor of Philosophy

at the University of Leicester

By

Mariel Voutounou

October 2012

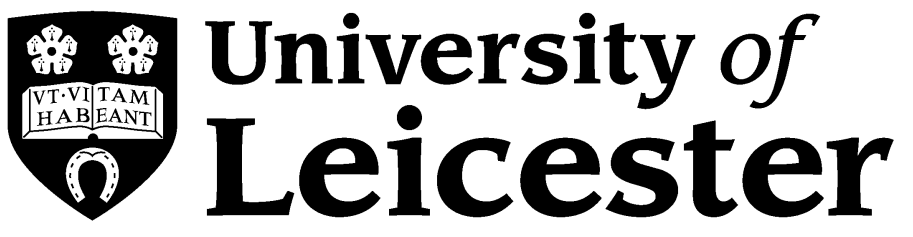


DOCTOR OF PHILOSOPHY (2012)

(GENETICS)
LEICESTER UNIVERSITY

UNITED KINGDOME

TITLE: THE EFFECTS OF METHYL-DONOR DEFICIENCY ON MUTATION INDUCTION AND TRANSGENERATIONAL INSTABILITY IN MICE

AUTHOR: MARIEL VOUTOUNOU

B.Sc. (Leicester University), M.Sc. (Leicester University)

SUPERVISOR: PROFESSOR YURI E. DUBROVA

NUMBER OF PAGES: 267

NUMBER OF WORDS: 43.597 


\section{The effects of methyl-donor deficiency on genome stability.}

Mariel Voutounou

Data from various recent human and animal studies link epigenetic changes caused by dietary deficiency of methyl donors like folate with many common human diseases, including cancer (Esteller, 2007; Feinberg, 2007). However, the mechanisms underlying the observed links between epigenetic alterations and disease remain elusive (Ames \& Wakimoto, 2002; Davis \& Uthus, 2004). Since the development of cancer is a multistep process in which somatic cells acquire mutations in a specific clonal lineage (Loeb et al., 2003), it appears plausible that hypomethylation following dietary restriction may destabilise the genome of treated animals.

To determine whether a methyl-donor deficient diet can cause longterm changes in the mutation rates of treated animals, BALB/c male mice were kept on a synthetic diet lacking in choline and folic acid for a duration of 8 weeks, directly after weaning. Sperm tissue was collected and analysed either at the end of treatment or 6 and 10 weeks after the end of dietary restriction. Single-molecule PCR technique was used to establish the frequency of mutation at the mouse expanded simple tandem repeat (ESTR) locus Ms6-hm in the treated males as well as their age-matched control groups. The results of this study showed that 8-weeks of dietary restriction did not affect ESTR mutation rate in the germline of treated male mice.

Given that the epigenetic landscape of mammalian cell is not fixed and undergoes massive reprogramming during development (Reik, 2007), it can potentially be affected by a variety of environmental factors (Cropley et al., 2006; Waterland et al., 2006). To investigate any transgenerational effects of methyl-donor deficiency on genome stability, sperm and brain tissues from the first-generation offspring of treated animals were also analysed. The frequency of ESTR mutation in the offspring of treated males did not significantly differ from that in controls, therefore implying the lack of measurable transgenerational effects of paternal dietary restriction. Taken together, the results of this study suggest that the effects of methyl-donor deficient diet on mutation induction and transgenerational instability in mice are likely to be negligible (Voutounou et al., 2012). 


\section{Acknowledgements:}

I would like to express the deepest respect and gratitude to my supervisor, Professor Yuri E. Dubrova for all the advice through the research phase and all guidance during the write-up of this thesis.

I would like to thank Dr. Colin D Glen for all his encouragement and support that kept me going and motivated. His knowledge and expertise were invaluable throughout the months of laboratory work.

I would like to thank the Division of Biomedical Services, University of Leicester for their expert animal care and the funding bodies Cancer Research UK (C23912/A12262), Welcome Trust (091106/Z/10/Z) and European Commission (NOTE, Contract Number 036465), whose grants made my work possible.

I would like to thank all G7 lab colleagues Dr. Ruth Barber, Dr. Ade Adeolou, Safeer Mughal, Andre Gomez and Hamdey Abouzeid Ali, for making the lab a fun place to work.

I would like to thank Dr. Roger Snowden and the MRC toxicology Unite in Leicester, for providing the equipment for the Pig-A assay.

Above all, I would like to thank my family for their love and constant support, which enabled me to complete this work. 


\section{Contents}

Page No:

Abbreviations

Chapter 1: Introduction

1.1. Folate 1

1.1.1. Importance of nutrients 1

1.1.2. Classical nutrient acute deficiency diseases and cancer 3

$\begin{array}{ll}\text { 1.1.3. Folate } & 6\end{array}$

1.1.4. Biochemical role of folate 9

1.1.4.1. Nucleotide synthesis 10

$\begin{array}{ll}\text { 1.1.4.2. Methylation cycle } & 12\end{array}$

$\begin{array}{ll}\text { 1.1.5. Folate deficiency } & 15\end{array}$

1.1.6. Uracil misincorporation and DNA stand breaks 17

1.1.7. Folate deficiency in rodents $\quad 20$

1.1.8. Folate deficiency and male fertility 24 


\subsection{Methyl donors and DNA methylation}

1.2.1. Epigenetic regulation 26

1.2.2. DNA methylation 28

1.2.2.1. De novo methylation 31

1.2.2.2. Maintenance methylation 33

1.2.2.3. Mechanisms of DNA demethylation 36

1.2.2.3.1. Active DNA hypomethylation 36

1.2.2.3.2. Replication-coupled DNA hypomethylation 38

1.2.3. Methionine importance to DNA methylation 41

1.2.4. Choline importance to DNA methylation 43

1.2.5. Folate importance to DNA methylation 48

1.2.6. DNA methylation and cancer

1.2.6.1. DNA hypomethylation 56

1.2.6.2. DNA hypomethylation and cancer 60

1.3. Cancer Risk 63

1.3.1. Folate link to carcinogenesis 64

1.3.2. Epidemiological evidence 68

$\begin{array}{ll}\text { 1.3.3. In-vitro studies } & 78\end{array}$

1.3.4. In-vivo animal studies $\quad 81$ 
1.4. Transgenerational instability

1.4.1. Epigenetic reprogramming 89

1.4.1.1. Epigenetic reprogramming during gametogenesis $\quad 89$

1.4.1.2. Epigenetic reprogramming during fertilisation 90

1.4.1.3. Evidence for histone domains retained in sperm 94

1.4.2. Transgenerational instability 95

1.5. Mutation Detection 100

$\begin{array}{ll}\text { 1.5.1. Traditional approaches } & 101\end{array}$

1.5.2. Repetitive DNA markers of mutation rates 102

1.5.3. Expanded simple tandem repeat (ESTR) loci 103

1.5.4. ESTR mutation induction 104

1.5.5. ESTR technique 110

$\begin{array}{ll}\text { 1.5.6. Tissue selection } & 112\end{array}$

1.5.7. Mosaic and singleton mutations 114

1.5.8. In vivo gene mutation assays 116

1.5.9. Pig-A assay

1.6. Aims and Objectives 
Chapter 2: Materials and methods

2.1. Materials

2.1.1. Animals 126

2.1.2. Experimental Design 126

2.1.3. Single molecule PCR technique

2.1.3.1. Chemical and molecular biology reagents

2.1.3.2. Radiochemical reagents

2.1.3.3. Enzymes

2.1.3.4. Oligonucleotides

131

2.1.3.5. Molecular weight markers

2.1.3.6. Standard solutions

2.1.3.7. Equipment

2.1.3.8. Computer software

2.1.3.9. Statistical analysis 


\subsection{Methods}

2.2.1. Single molecule PCR technique

2.2.1.1. Tissue Collection

2.2.1.2. DNA extractions

2.2.1.2.1. Brain DNA extraction

2.2.1.2.2. Sperm DNA extraction

2.2.1.3. DNA digestion and precipitation

2.2.1.4. DNA quantification

2.2.1.5. Dilution of samples for S.M PCR optimization

138

2.2.1.6. Polymerase Chain Reaction

2.2.1.7. Gel for Single- Molecule PCR optimization

2.2.1.8. Southern Blotting

2.2.1.9. HM1 probe construction

2.2.1.10. Radioactive labelling of probe and recovering

2.2.1.11. Hybridization

2.2.1.12. Washing Membranes

2.2.1.13. Autoradiography

2.2.1.14. Developing films

2.2.1.15. Evaluating single molecule concentration

2.2.1.16. Single molecule PCR

2.2.1.17. Identification of positive PCR reactions

2.2.1.19. Southern blotting long gels 
2.2.2.1. Blood Harvest 146

$\begin{array}{ll}\text { 2.2.2.2. Cell staining } & 146\end{array}$

$\begin{array}{ll}\text { 2.2.2.3. Flow cytometric analysis } & 147\end{array}$

$\begin{array}{ll}\text { 2.2.2.4. Data acquisition } & 147\end{array}$

\section{Chapter 3: Results}

$\begin{array}{ll}\text { 3.1. S.M. PCR Technique } & 148\end{array}$

3.1.1. Single molecule-PCR optimisation 148

3.1.2. Poisson analysis 149

3.1.3. Mutation Scoring 150

3.1.4. Mutation mosaicism 152

3.1.5. ESTR mutation frequencies in control groups 153

3.1.6. Effects of dietary methyl deficient diet on ESTR mutation induction

3.1.7. Transgenerational effects of paternal methyl donor deficiency

3.1.8. Effects of a methyl-donor deficiency on ESTR mutation spectrum

3.2.1. Method of optimisation 173

3.2.2. Effects of methyl deficient diet on Pig-A mutation rate 179 
Chapter 4: Discussion

4.1. Summary of results

182

4.2. Effects of diet on genome stability

183

4.3. Effects of diet on methylation status

4.4. Analysis of results

4.4.1. ESTR mutation frequencies in control groups

4.4.2. Effects of methyl-donor deficiency on ESTR mutation induction

4.4.3. Transgenerational effects of paternal methyl-donor deficiency

4.4.4. Effects of a methyl-donor deficient diet on ESTR mutation spectrum

4.5. Phenotypic changes in somatic tissue $v$ germline effects

203

4.6. Pig-A assay 205

4.7. Conclusion 206

4.8. Future work 


\section{Appendix}

5.1. Methyl donor diets 209

5.1.1.Methyl sufficient diet 209

5.1.2. Methyl deficient diet $\quad 211$

5.2. Summary of ESTR data 213

5.2.1. All ESTR mutation data: $F_{0}$ sperm 213

5.2.2. All ESTR mutation data: $F_{1}$ sperm 220

5.2.3. All ESTR mutation data: $F_{1}$ brain 224

5.3. Summary of Pig-A data 228

5.3.1. All ENU, Control and Treated $F_{0}$ data 228

5.3.2. All Control and Treated $F_{1}$ data 230

5.3.3. All $F_{0}$ and $F_{1}$ combined data 231

References 
Abbreviations:

AdoMet Adenosylmethionine

Al Adequate intake

ATP Adenosine triphosphate

ATRX Alpha-thalassemia X-linked mental retardation syndrome

Avy Agouti viable yellow gene

Axin1Fu Axin 1 fused gene

bp $\quad$ Base pair

BSA Bovine serum albumin

BLCLs B-lymphoblastoid cell lines

BRCA1 Breast cancer 1 tumor suppressor gene

BRCA2 Breast cancer 2 tumor suppressor gene

$\mathrm{CH}_{2}$ THF Methylene-THF

$\mathrm{CH}_{3}$ THF 5-methyl-THF

CIR Circular nuclei

CNS Central nervous system

CRA Colorectal adenomas

CRC Colorectal cancer

CVD Cardiovascular disease

DFI DNA fragmentation index

DHF Dihydrofolate

DM1 Myotonic dystrophy type 1

DMBA 7,12-dimethyl-1,2-benz[a]anthracene

DNA Deoxyribonucleic acid

DNMT DNA methyl-transferase 
dNTP Deoxynucleoside tri-phosphate

DRI Dietary reference intake

DSB Double strand break

dsRNAs Double stranded RNAs

dTMP Deoxythymidine monophosphate

dTTP Deoxythymidine tri-phosphate

dUMP Deoxyuridine monophosphate

EAR Estimated average intake

EDTA Ethylene diamine tetraacetic acid

ELS Early life stress

ENU N-ethyl N-nitrosourea

ESTR Expanded simple tandem repeat

EtBr Ethidium bromide

EtOH Ethanol

FA Folic acid

FAB Familial adenomatous polyposis

FAD Flavin adenine dinucleotide

FCM Flow cytometry

FD Folic acid deficiency

FUS $\quad$ Fused nuclei

g

Gram

GPA Glycophorin A

GPI Glycosyl-phophatidyl-inositol

HATs Histone acetyl transferases

HCl Hydrochloric acid 
HD Huntington's disease gene

HDACs Histone deacetylases

HIV Human immunodeficiency virus

HNSCC Head and neck squamous cell carcinoma

HPTR hypoxanthine phosphoribosyltransferase gene

HPV Human papilloma virus

HS Horseshoe nuclei

HWS High stringency wash solution

$\mathrm{H}_{2} \mathrm{O} \quad$ Water

hr(s) Hour(s)

IAP Intracisternal A-particle elements

IR lonising Radiation

$\mathbf{K}^{+} \quad$ Sodium

$\boldsymbol{k} \quad$ Total number of reactions

I Litre

ICF Immunodeficiency centromere instability and facial anomalies syndrome

JmjC Jmonnji domain histone demethylases

LET Linear transfer energy

LINE Long interspersed nucleotide elements

LSD1 Lysine-specific demethylase 1

Lsh Lymphoid-specific helicase

Kb Kilo base pair

m Milli

M Molar 
$\begin{array}{ll}\text { MAT } & \text { Methionine adenosyltransferase } \\ \text { MBD } & \text { Methyl-CpG-binding domain protein }\end{array}$

MeCP2 Methyl-CpG-binding protein 2

MeDIP Methylated DNA immunoprecipitation

$\min (\mathbf{s}) \quad$ Minute(s)

MMA Methylmalonic acid

$\mathrm{Mn}^{2+} \quad$ Divalent manganese ion

MN Micronucleus expression

MTA Methylthioadenosine

MTHFR Methylene tetrahydrofolate reductase

$\boldsymbol{n}_{+} \quad$ Number of positive reactions

n. Number of negative reactions

$\mathrm{Na}^{+} \quad$ Sodium

NaAc Sodium acetate

$\mathrm{NaCl} \quad$ Sodium chloride

NAD Nicotinamide adenine dinucleotide

NADP Nicotinamide adenine dinucleotide phosphate

$\mathrm{NaHPO}_{4} \quad$ Sodium hydrogen phosphate

$\mathrm{Na}_{2} \mathrm{PO}_{4} \quad$ Sodium phosphate

Nat2 N-acetyltransferase 2 gene

ncRNAs Non-coding RNAs

NG Nitrosoguanidine

NHEJ Non-homologous end joining

NTD Neural tube defect

OLB Oligo labeling buffer 
OSS Oligo Stop Solution

p Pico

$\boldsymbol{P}_{+} \quad$ Frequency of positive reactions

$\boldsymbol{P} \quad \quad$ Frequency of negative reactions

PCR Polymerase chain reaction

PEMT Phosphatidylethanolamine N-methyltransferase

PGCs Primodial germ cells

Pig-A Phosphatidylinositol glycan complementation, group A

PLP Pyridoxal phosphate

PNH Paroxysmal nocturnal hemoglobinuria

Prob. Probability

PSEN1 Presenilin 1 gene, involved in CNS development

PSEN2 Presenilin 2 gene, important for healthy brain function

PWS Phosphate wash solution

RBCs Red blood cells

RDA Recommended daily allowance

RETs Reticulocytes

RISC RNA-induced silencing complex

ROS Reactive oxygen species

RNA Ribonucleic acid

RNAi RNA interference

SAH S-adenosylhomocysteine

SAM S-adenosylmethionine

SHMT Serine hydroxymethyltransferase

SCO Sertoli cell only syndrome 
SDS Sodium dodecyl sulphate

SINE Short interspersed nucleotide elements

siRNAs Small interfering RNAs

SM-PCR Single molecule polymerase chain reaction

SSB Single Strand Break

SSC Sodium chloride and sodium citrate buffer

Taq Thermus aquaticus

TBE Tris borate EDTA

TGCT Testicular germ cell tumor

THF Tetrahydrofolate

TS Thymidylate Synthetase

TSA Trichostatin A

U Unit(s)

UL $\quad$ Upper limit

Ung Uracil-DNA glycosylase

UV Ultra-violet

V Volt(s)

${ }^{\circ} \mathrm{C} \quad$ Degree celsius

$\lambda \quad$ Average number of amplifiable molecules

$\boldsymbol{\mu} \quad$ Micro

$\boldsymbol{\mu} \quad$ Mutation rate 


\section{Chapter 1. Introduction}

\subsection{Folate}

\subsubsection{Importance of nutrients}

Vitamins, essential minerals and other compounds (in the region of 40 micronutrients) are essential in the human diet for a healthy metabolism, normal growth and development (Ames, 2001). The majority of vitamins cannot be synthesized by the human body and must be taken by food consumption. The most known vitamins are $B_{1}$ (thiamine), $B_{2}$ (riboflavin), $B_{3}$ (niacin), $\mathrm{B}_{5}$ (pantothenic acid), $\mathrm{B}_{9}$ (folic acid), $\mathrm{B}_{12}$ (cobalamin) and $\mathrm{C}$ (ascorbic acid). There are two types of vitamins; the fat-soluble and the water-soluble (Ellis \& Macleod, 1922; Nestle, 2002). The fat-soluble vitamins are $A, D, E$ and $K$, and the main source of fat-soluble vitamins is meat, animal fat, dairy products, fish and vegetable oils. The way these vitamins are transported around the body is in fat and any excess found is stored in the liver and fatty tissues. Therefore, it is not essential to obtain these vitamins from food resources daily. The water-soluble vitamins are B and C, and the main sources of water-soluble vitamins are fruits, vegetables, wholegrains, fish and meat. These water-soluble vitamins are transported around the body in water. They cannot be stored, as any excess is discarded with urine, therefore they must be attained from food consumption daily. It must be noted that cooking destroys water-soluble vitamins (Brandt \& Rutherford, 2007).

As with vitamins, adequate consumption of minerals is also essential for the proper function of the human body, particularly due to their role as building blocks for muscles, soft tissue and bones. They are also vital for 
many life supporting systems such as hormones, nerve response, oxygen transport, heartbeat, blood clotting and enzyme systems. Minerals are categorized into two groups: (a) major and (b) trace minerals. This classification merely indicates the difference in quantity by which the minerals are needed and not their importance, as all are vital for the body's functions (Sandeman, 2000; Ellis \& Macleod, 1922).

Many vitamins such as vitamins $A, C$, and $E$ and minerals such as zinc, copper, selenium, or manganese act as antioxidants, protecting the organism from free radicals and their harmful effects to the body. This is due to their ability to trace highly reactive radicals and transform them into inactive compounds reducing their damaging ability, thus preventing cancer and other harmful degenerative diseases, like premature aging, heart and autoimmune diseases, Alzheimer's disease, and diabetes mellitus (Farlex, 2010).

There is a daily optimal intake for each vitamin and micronutrient in order to achieve metabolic harmony and maximum life span, and it is defined by the recommended daily allowance (RDA). The RDA is the average daily dietary intake level that is sufficient to meet the nutrient requirements of nearly all (approximately 98\%) healthy individuals. Similar to RDA is the dietary reference intake $(\mathrm{DRI})$, the adequate intake $(\mathrm{Al})$, the estimated average intake (EAR) and the tolerable upper limit (UL) for each micronutrient. As the optimum amounts for each micronutrient, for long-term health are still by and large unknown, the RDA of micronutrients is predominantly based on data on acute effects (Ames, 2001; Nestle, 2002). 


\subsubsection{Classical nutrient acute deficiency diseases and cancer}

Inadequate intake of some vitamins produces easily recognized acute deficiency diseases like scurvy (lack of ascorbic acid), beriberi (lack of thiamine), pernicious anaemia (lack of $B_{12}$ ), rickets (vitamin D deficiency) and pellagra (lack of niacin). In developed countries these acute deficiencies are very rare, but suboptimal nutrient intake; less than the RDA, is a common and pervasive problem (Ames \& Wakimoto, 2002).

Data from population studies obtained from the past twenty years revealed that a substantial percentage of the population lacks in various micronutrients in relation to the current RDA (Wilson et al., 1997). The findings are very concerning considering the fact that micronutrient deficiencies interfere with metabolic balance and have been frequently linked to impairment of DNA integrity. Micronutrient deficiencies have been found to imitate the effects of radiation and chemicals, in their aptitude to damage DNA, causing single and double-strand breaks and lesions, which are a major cause of cancer. The micronutrients whose deficiency has been found to mimic the effects of radiation are folic acid, $B_{12}, B_{6}$, niacin, $C, E$, iron, and zinc (Bonassi et al., 2000). However the corresponding level to which each micronutrient minimises DNA damage is yet to be established. With little expenditure these deficiencies could be easily rectified resulting in dramatically improving health and therefore life span (Ames, 2001).

The daily-recommended consumption of fruits and vegetables is 5 portions, and micronutrient deficiencies could explain the piling epidemiological evidence linking little intake of fruits and vegetables with cancer. Studies in the American population show that $80 \%$ of American 
children (Krebs-Smith et al., 1995) and 68\% of adults consume less than the recommended daily portion of fruits and vegetables (Krebs-Smith et al., 1996). A plethora of epidemiological studies associate low fruit and vegetable consumption with cancer, cardiovascular diseases and brain deficiencies (Block et al., 1992; Steinmetz \& Potter, 1996; Willett \& Trichopoulos, 1996; Chatenoud et al., 1998; Jacobs et al., 1998). It has been shown that nonsmoker groups that are mostly vegetarian have about half the cancer mortality rate and a longer life span than the average person (Mills et al., 1994).

As Ames states, the reason why the quarter of the population that consumes the fewest fruits and vegetables was found to have approximately double cancer rates for the majority of cancer types (lung cancer, larynx cancer, oral cavity cancer, esophagus cancer, stomach cancer, colon and rectum cancer, bladder cancer, pancreatic cancer, cervix cancer, and ovarian cancer) when compared to the quarter with the highest fruit and vegetable consumption, could be explained by micronutrient deficiencies.

Table 1.1.1 shows the recommended daily allowance for a number of micronutrients. Many factors, including aspects such as age, genetic predisposition and health-state, could play an important role in the optimum intake of a micronutrient that each individual needs. The determination of these optimizations (in the form of a public health project) is one of the major issues that need addressing in the impending future (Ames, 2001). 
Table 1.1.1 Micronutrient deficiencies in US population.

\begin{tabular}{|c|c|c|c|c|}
\hline Nutrient & $\begin{array}{l}\text { Population } \\
\text { group }\end{array}$ & $\begin{array}{l}\text { Current } \\
\text { RDA }\end{array}$ & $\begin{array}{l}\text { \% Consuming } \\
\text { less than the } \\
\text { RDA }\end{array}$ & $\begin{array}{l}\text { \% Consuming } \\
\text { less than half } \\
\text { the RDA }\end{array}$ \\
\hline Minerals & & & & \\
\hline Iron & $\begin{array}{l}\text { Women } \\
20-30 \text { years }\end{array}$ & $18 \mathrm{mg}$ & $75 \%$ & $25 \%$ \\
\hline & $\begin{array}{l}\text { Women } 50 \\
\text { years }\end{array}$ & $8 \mathrm{mg}$ & $25 \%$ & $5 \%$ \\
\hline Zinc & $\begin{array}{l}\text { Women/men } \\
\text { 50+years }\end{array}$ & $8-11 \mathrm{mg}$ & $50 \%$ & $10 \%$ \\
\hline Vitamins & & & & \\
\hline Folate & $\begin{array}{l}\text { Women } \\
20+\text { years }\end{array}$ & $400 \mu \mathrm{g}$ & $75 \%$ & $50 \%$ \\
\hline & Men 20+ years & $400 \mu g$ & $75 \%$ & $25 \%$ \\
\hline $\mathrm{B}_{6}$ & $\begin{array}{l}\text { Women/men } \\
20+\text { years }\end{array}$ & $1.5-1.7 \mathrm{mg}$ & $50 \%$ & $10 \%$ \\
\hline $\mathrm{B}_{12}$ & $\begin{array}{l}\text { Women } \\
20+\text { years }\end{array}$ & $2.4 \mu g$ & $25 \%$ & $10 \%$ \\
\hline & Men 20+ years & $2.4 \mu g$ & $10 \%$ & $5 \%$ \\
\hline $\mathrm{C}$ & $\begin{array}{l}\text { Women/men } \\
20+\text { years }\end{array}$ & $75-90 \mathrm{mg}$ & $50 \%$ & $25 \%$ \\
\hline
\end{tabular}

Adapted from Ames \& Wakimoto 2002. 


\subsubsection{Folate}

Vitamin $\mathrm{B}_{9}$, also known as folate, is an essential water-soluble $\mathrm{B}$ vitamin needed by the body to synthesize, repair and methylate DNA. The human body cannot synthesize folate; therefore it is obtained from dietary sources. It occurs naturally in a variety of foods, especially leafy vegetables such as cabbage, cauliflower and broccoli, and in many fruits and nuts (Bailey \& Ayling, 2009).

Dietary folate has a molecular formula of $\mathrm{C}_{12} \mathrm{H}_{19} \mathrm{~N}_{7} \mathrm{O}_{6}$ and a molecular mass of 441.4. The term 'folate' encompasses all one-carbon substituted and glutamylated derivatives of folic acid (Christensen et al., 2011). Folic acid is folate's synthetic oxidized form and is used in fortified foods and vitamin supplements, since it is more stable (Beetstra et al., 2005). Folic acid is made up of three parts: a pteridine ring system (6-methylpterin), a paraaminobenzoic acid and a glutamic acid (Figure 1.1.1).

Folic acid in the interior of cells usually contains a chain of several glutamic acids (usually 3 to 8 ), whose role is to act as a negatively charged handle, either keeping the coenzyme inside the cell or bounding it to appropriate enzymes. The glutamic acid is not involved in the folic acid functions, whereas the pteridine and p-aminobenzoic acid portions of the coenzyme participate directly in the metabolic reactions of folate. 
Figure 1.1.1. Folic acid structure.

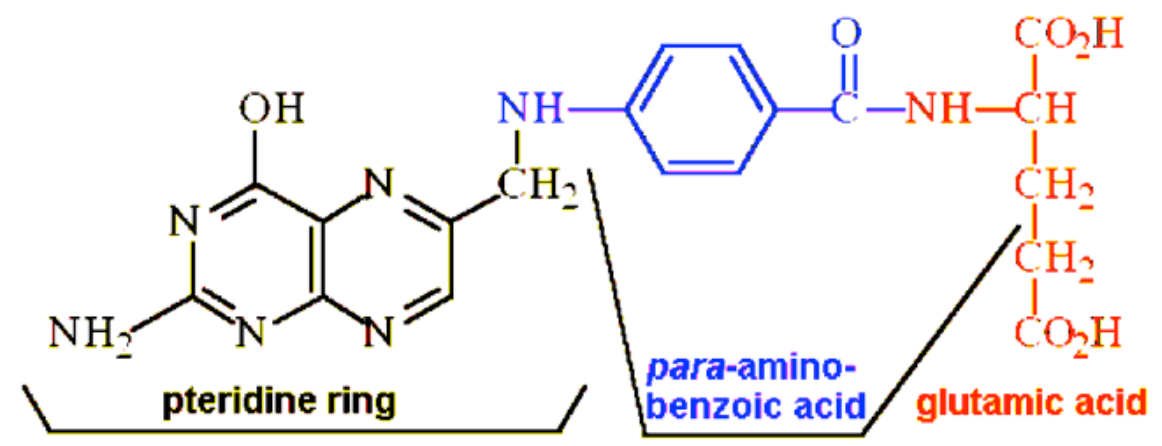

Adapted from http://themedicalbiochemistrypage.org/images/folate.jpg.

Folate is a cofactor in a plethora of biochemical reactions due to its ability to donate or accept one-carbon units (Duthie, 1999). It is especially important during periods of rapid cell division and growth, and both children and adults need folate for the production of healthy red blood cells (Kim 2004; Bailey \& Ayling, 2009).

Sufficient folate intake is essential for normal cell division and homeostasis, as folate coenzymes are necessary for nucleic acid synthesis and methionine regeneration. Folate is therefore essential for the support of DNA synthesis and cell proliferation and it is vital for the transportation, oxidation and reduction of one-carbon units, important for normal cell metabolism and regulation.

The amount of folate coenzymes in an organism mainly depends on (a) how much folate is consumed with food, and (b) the rate folate is lost through catabolic reactions as well as urinary and fecal routes. In periods when the organism is deprived of folate either due to inadequate folate intake or mall absorption, biochemical changes linked with folate insufficiency take place, permitting the beginning of malfunction in the one-carbon metabolism (Bailey \& Gregory, 1999). 
Abnormalities such as hyperhomocysteinemia or DNA hypomethylation (Jacob et al., 1998) are a result of such malfunctions in the one-carbon metabolism and could lead to a number of deleterious effects such as a greater risk for specific chronic diseases (Boushey et al., 1995), developmental disorders such as neural tube defects and an increase in cancer risk (Daly et al., 1995; Daly et al., 1997). Indeed recent experimental evidence support the idea that folate deficiency could lead to both genetic and epigenetic changes that either initiate or promote the development of cancer by mechanisms discussed in Sections 1.1.6 and 1.2.5. On the contrary, excess quantities of folate could enhance the proliferation of neoplastic cells, thereby accelerate tumor growth (Christensen et al., 2011).

The long-standing objective in determining folate requirements entails finding out the necessary intakes that decrease the harmful processes linked with insufficiency or excess in folate consumption and thus successfully optimize all metabolic and cellular reactions that are folate-reliant (Bailey \& Gregory, 1999). The current RDA for folate is shown in Table 1.1.2. 
Table 1.1.2. Recommended dietary allowance (RDA) for folate.

\begin{tabular}{|l|l|c|c|}
\hline Life stage & Age & Males $(\boldsymbol{\mu g} /$ day) & Females ( $\mu$ g/day) \\
\hline Infants & $0-6$ months & 65 & 65 \\
\hline Infants & $7-12$ months & 80 & 80 \\
\hline Children & $1-3$ years & 150 & 150 \\
\hline Children & $4-8$ years & 200 & 200 \\
\hline Children & $9-13$ years & 300 & 400 \\
\hline Adolescents & $14-18$ years & 400 & 400 \\
\hline Adults & $19+$ years & 400 & 600 \\
\hline Pregnancy & All ages & - & 500 \\
\hline Breast-feeding & All ages & - & \\
\hline
\end{tabular}

Adapted from Bailey \& Gregory, 1999.

\subsubsection{Biochemical role of folate}

All folate-requiring reactions are referred to as 'one carbon metabolism' and include the amino acid-metabolism, the purine and pyrimidine synthesis and the formation of the primary methylating agent, Sadenosylmethionine (SAM) described in (Bailey \& Gregory, 1999; Hazra et al., 2007).

Folate has two essential carbon-carbon double bonds and as mentioned above, its main role is to accept or donate single carbon units in key metabolic pathways (Wagner, 1995). It is not by itself biologically active but it is converted into the liver to dihydrofolate (DHF) via saturation of one of its carbon-carbon double bonds, and then to tetrahydrofolate (THF) with an addition of an extra hydrogen molecule across the second carbon-carbon 
double bond. These active derivatives are essential to nucleotide biosynthesis and the re-methylation of homocysteine (Kim, 2004; Hazra et al., 2007).

THF is the central folate acceptor molecule in the one carbon cycle (Hazra et al., 2007). It is an enzyme cofactor whose role is to transport onecarbon units needed in several biosynthetic reactions. One-carbon units are transferred to and from THF, inter-converted between several oxidative states and employed in the synthesis of nucleotides and amino acids. Folatemediated one-carbon metabolism can be simplistically divided into two sections: the nucleotide synthesis and the methylation cycle (Christensen et al., 2011).

\subsubsection{Nucleotide synthesis}

The first section of the folate mediated-one carbon metabolism is the nucleotide synthesis. Three out of four nucleotides in DNA need folate for their de novo synthesis. Synthesis of thymidylate (dTMP) from uridylate (dUMP) is achieved by thymidylate synthase (TS) and requires carbon units from 5,10-methyleneTHF. On the other hand the multi-step synthesis of the purine nucleotides adenylate and guanylate requires two molecules of formylTHF per nucleotide (Christensen et al., 2011).

Conversion of THF to 5,10-methylene-THF is therefore an essential first step in the one carbon cycle that utilizes the 3-carbon of serine as a major carbon source. This one-carbon unit is passed on from serine to THF via pyridoxal phosphate (PLP)-dependent serine hydroxymethyltransferase (SHMT) to form 5,10-methyleneTHF and glycine (Bailey \& Gregory, 1999; 
Hazra et al., 2007).

The donor 5,10-methyleneTHF $\left(\mathrm{CH}_{2} \mathrm{THF}\right)$ is used directly in thymidylate synthesis (along with a molecule of hydrogen at the site of one of the double bonds) by donating its $\mathrm{CH}_{2}$ unit and thus converting dUMP (deoxyuridylate) into dTMP (thymidylate). By donating its $\mathrm{CH}_{2}$ unit, 5,10methyleneTHF becomes the thymidine methyl group and thus synthesizes dTMPs that are strictly used for DNA synthesis (DNA repair and DNA replication) (Bailey \& Gregory, 1999; Hazra et al., 2007).

Finally the donor 10 -formylTHF (CHOTHF) is used directly in purine synthesis to set up ring closure reactions in the de novo purine synthesis. Purines are very important as they are utilized both in RNA and DNA synthesis (Bailey \& Gregory, 1999; Hazra et al., 2007).

A part of the 5,10-methylenetetrahydrofolate undergoes irreversible enzymatic reduction to the methyl oxidation state (as 5-methylTHF) by the enzyme methylene tetrahydrofolate reductase (MTHFR). The resulting $\mathrm{N}-5$ methyl group of 5-methylTHF can only be used metabolically for transfer to homocysteine, resulting in the re-generation of methionine by the MTHFR enzyme (Bailey \& Gregory, 1999).

The role of folate in the synthesis of the amino acid methionine is particularly important, as methionine generates the ubiquitous methyl donor S-adenosylmethionine (SAM), which is used in the transfer of methyl groups in a plethora of reactions, such as DNA and protein methylation, as well as phospholipid and neurotransmitter synthesis (Christensen et al., 2011). 


\subsubsection{Methylation cycle}

The second section of the folate mediated one-carbon metabolism is the methylation cycle. The two sections are bridged via the MTHFR enzyme, which as mentioned above, catalyses the irreversible reaction of 5,10 methyleneTHF to 5-methylTHF, committing one-carbon folates to the methylation cycle (Bailey \& Gregory, 1999; Christensen et al., 2011).

MTHFR plays a vital role in the methylation cycle, as it converts methylene-THF to the single carbon donor 5-methyl-THF $\left(\mathrm{CH}_{3} \mathrm{THF}\right)$. Later on, the methyl groups are transferred from 5-methylTHF to the toxic amino acid homocysteine (Hcy). By that action, MTHFR permanently directs the products of the one carbon cycle to the methylation of homocysteine (Wagner, 1995). Re-methylation of homocysteine depends on the dietary content of methionine and choline, and usually $50-80 \%$ of generated homocysteine is remethylated (Bailey \& Gregory, 1999; Bistulfi et al., 2010; Christensen et al., 2011).

During the methionine synthase (MS) reaction, 5-methylTHF loses a methyl group, which works as a substrate, and is consecutively transported to the vitamin $B_{12}$ coenzyme before homocysteine, thus forming methionine (Bailey \& Gregory, 1999). Additionally to protein synthesis, methionine can be then used to methylate DNA, as it is the main methyl-group donor for processes including DNA methylation reactions. Methionine does so by donating methyl groups through conversion to S-adenosylmethionine (SAM) (Bistulfi et al., 2010).

SAM is an essential biological methylating agent, both for its involvement in the biosynthesis of polyamines (spermidines and spermines) 
and its key role in the methyltransferase reactions, with numerous acceptor molecules that involve DNA, RNA, and proteins including histones (Bailey \& Gregory, 1999; Bistulfi et al., 2010). When SAM participates in methylation reactions like DNA methylation or phospholipid synthesis, it generates Sadenosylhomocysteine (SAH), which is, in turn, metabolized to produce Hcy and complete the cycle (Christensen et al., 2011).

Additionally, the MS reaction regenerates tetrahydrofolate (THF), which is needed for the formation of all three oxidation states; 5-methyl-THF ( $\mathrm{CH}_{3}$ THF- reduced), 5,10 methylene-THF $\left(\mathrm{CH}_{2}\right.$ THF- intermediate $)$ and 10formyl-THF (CHOTHF-oxidized) to all of which folate acts as a single carbon donor. Concluding to the above, the folate pathway is central and thus essential for DNA methylation, dTMP synthesis or purine synthesis (Bailey \& Gregory, 1999; Hazra et al., 2007).

The key role of folate in the one-carbon metabolism, methionine cycle and polyamine biosynthesis is illustrated in Figure 1.1.2. 


\section{Figure 1.1.2. Role of folate in one-carbon metabolism.}

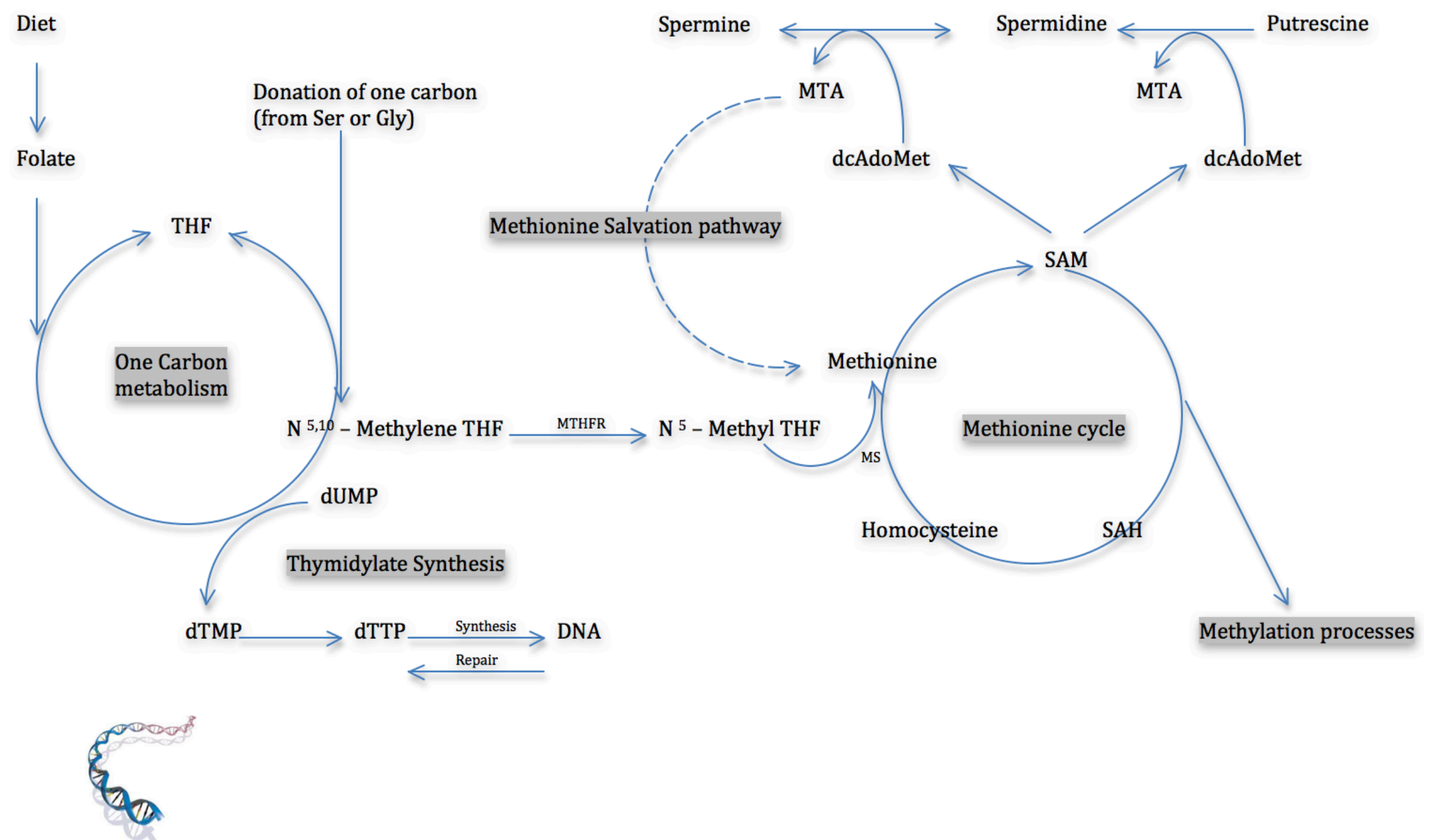

The pathway by which folate is used as a methyl donor for the production of 5' methylcytosine (DNA Methylation) and deoxy thymidine monophosphate (dTMP). Adapted from Fenech, 2001. 


\subsubsection{Folate deficiency}

As mentioned above, the current adult RDA for folate is $400 \mu \mathrm{g}$. However, due to poor eating habits, insufficient folate intake is a persistent problem, in a significant percent of the human population. Lack or low intake of folate results in folic acid deficiency (FD) which if left untreated can cause birth defects such as neural tube defects in developing embryos, like Spina bifida cleft lip (Czeizel \& Dudas, 1992), nonsyndromic clef lip palate and craniofacial defects (Van Rooij et al., 2004).

Folate deficiency is also linked to preterm delivery, low birth weights due to slow in utero growth and a relatively high amount of spontaneous abortions (Scholl \& Johnson, 2000). As a result of these findings, during pregnancy women are advised to receive more than $600 \mu \mathrm{g}$ per day.

Additionally, FD has been linked to anaemia in children and young adults. A study conducted by Thompson and co-authors revealed that folate deficiency could lead to acute lymphoblastic leukaemia, which is the most prevalent childhood cancer (Thompson et al., 2001). According to a population study conducted by Ames in $2001,75 \%$ of the US population consumed less folate than the RDA, from which $25 \%$ of men and $55 \%$ of women consume even less than half the recommended daily allowance (Table 1.1.1). The study further revealed that $10 \%$ of the population tested was folate deficient to a point that caused DNA lesions and chromosomal breaking and that this percentage was even higher in poorer regions (Ames, 2001).

The damage caused by folate and other micronutrient deficiencies as Ames states, appears to be many times more important than the damage caused by radiation and chemicals, and curing them will hugely improve both 
human health conditions and life span. The mechanism that leads to DNA damage by causing single- and double-strand breaks is explained in Section 1.1.6 and employs the misincorporation of uracil instead of thymine into the DNA in a rate of four million per cell (Ames, 2001).

The results of many human studies show that high uracil levels in the DNA and DNA breakage can be reversed by folate administration (Blount et al., 1997). An Australian study showed that folate supplementation above the recommended daily allowance (RDA) minimizes chromosome breaks (Fenech et al., 1997) and a study observing the results of a 15-year use of a multivitamin supplement containing folate showed that adequate daily consumption of folate lowers the risk of colon cancer to approximately $75 \%$ (Giovannucci et al., 1998).

Due to the importance of adequate daily folate consumption, the food and drug administration (FDA) in the United States and Canada mandated fortification with folic acid to all cereal-grain foods, in order to increase the average intake of folate and reduce the incidence of neural tube defects (De Wals et al., 2007). Indeed, since the mandatory fortification of these foods in 1998 , the average intake of folate has increased by over $100 \mu g$ a day in the US population (Quinlivan \& Gregory, 2003).

Despite the increase in folate intake, a significant percentage of the population has undergone a period of folate deficiency and therefore it must be determined if this could have a long-term impact to the health of individuals, or any transgenerational effects in their children (Swayne et al., 2012a). Furthermore the impact of low folate levels should be analysed in other population groups, where no food fortification scheme took place and long-term folic acid deprivation is a persisting problem. 


\subsubsection{Uracil misincorporation and DNA strand breaks}

When uracil incorporates into the DNA, its usually due to the spontaneous hydrolysis of cytosine to uracil (a common event), which if not repaired could result in a $\mathrm{C}$ to $\mathrm{T}$ transition (Christensen et al., 2011). Low folate levels in a cell have been shown to lead to an abnormally high level of uracil misincorporation, caused by the decrease in methyleneTHF availability for the thymidylate synthase reaction (Figure 1.1.3).

As previously mentioned, the methylene-THF pool derives from folate, and is necessary for the methylation of deoxy-uridine monophosphate (dUMP) to deoxy-thymidine monophosphate (dTMP) by thymidylate synthetase (TS) (Ames \& Wakimoto, 2002; Christensen et al., 2011). Nucleotide pools in cells are only large enough to support DNA synthesis for a few minutes (Meuth, 1998), therefore altered dUTP:dTTP ratios caused by folate deficiency can rapidly affect DNA synthesis and repair (Christensen et al., 2011).

Misincorporated uracils in the DNA are detected by a number of base excision repair systems, such as glycosylase repair enzymes and they are usually excised and replaced with the proper nucleotide. In order to excise the miss-incorporated uracils, the glycosylase repair enzymes have to induce transient single-strand breaks in the DNA. Therefore, if large amounts of uracil are incorporated into the DNA, opposing single-strand breaks can occur, causing double strand chromosomal breaks that are highly mutagenic and are difficult for cells to repair (Dianov et al., 1991; Blount et al., 1997; Ames \& Wakimoto, 2002).

DNA double-strand breaks can result in chromosomal translocations, inhibition of gene expression, deletion of tumor suppressor genes, and 
amplification of oncogenes (Ciappio \& Mason, 2010). They have also been linked to elevated expression of tumor suppressor genes (Crott et al., 2007).

Experiments on cultured cells have shown that low folate levels increase uracil incorporation and DNA strand breaks (Duthie \& Hawdon, 1998; Duthie et al., 2000b; Crott et al., 2001). Moreover, folate deficiency was found to result in a reduction of the ability of cells to repair DNA damage (James et al., 1994; Duthie \& Hawdon, 1998; Duthie et al., 2000b). This decrease in DNA damage repair ability could further enhance the accumulation of mutations and other types of instability in the genome.

Numerous animal models confirm the in vitro findings, linking folate deficiency with increased dUTP:dTTP ratios, uracil incorporation, and DNA strand breaks caused by increased base excision repair activity (Pogribny et al., 1997; Choi et al., 1998; Duthie et al., 2000a; Knock et al., 2008). Uracil misincorporation is also linked to tumor development in mice, as data from folate-deficient BALB/c mice have shown an increase in dUTP:dTTP ratios and DNA strand breaks in intestinal tissues, that are known to increase the risk of intestinal tumor development (Knock et al., 2008).

In humans, evidence linking uracil misincorporation and tumorigenesis have been reported. Some studies have linked low folate blood levels with increased dUTP:dTTP ratios, uracil misincorporation and DNA breaks in lymphocytes (Blount et al 1997; Jacobs et al., 1998; Basten et al., 2006), while Hazra and co-authors reported a borderline association between uracil misincorporation and the recurrence of colorectal adenoma (CRA) (Hazra et al., 2010). However, a resent study failed to observe this relation in either lymphocytes or rectal mucosa (Christensen et al., 2011), while others equally failed to find any significant association between folate deficiency, DNA 
stability or chromosomal damage (Fenech et al., 1997; Basten et al., 2006). Therefore the link between folate deficiency, uracil misincorporation and DNA instability remains elusive.

It is worth noting that the relationship between folate and uracil misincorporation could be influenced by several genetic or environmental factors. Age might play a critical role, as uracil misincorporation due to folate deficiency was found to be enhanced in the colon of older rats (Choi et al., 2003). Uracil misincorporation can also be affected by genetic factors such as folate-related single nucleotide polymorphisms (SNPs), like the MTHFR 667 C->T variant (Knock et al., 2006; Knock et al., 2008; De Vos et al., 2008). Additionally, folate fortification (Hazra et al., 2010) and vitamin $B_{12}$ levels (Fenech 1998; Kapiszewska et al., 2005) could play an important role to the level of uracil misincorporation in the cells (Christensen et al., 2011).

Figure 1.1.3 Uracil misincorporation into DNA.

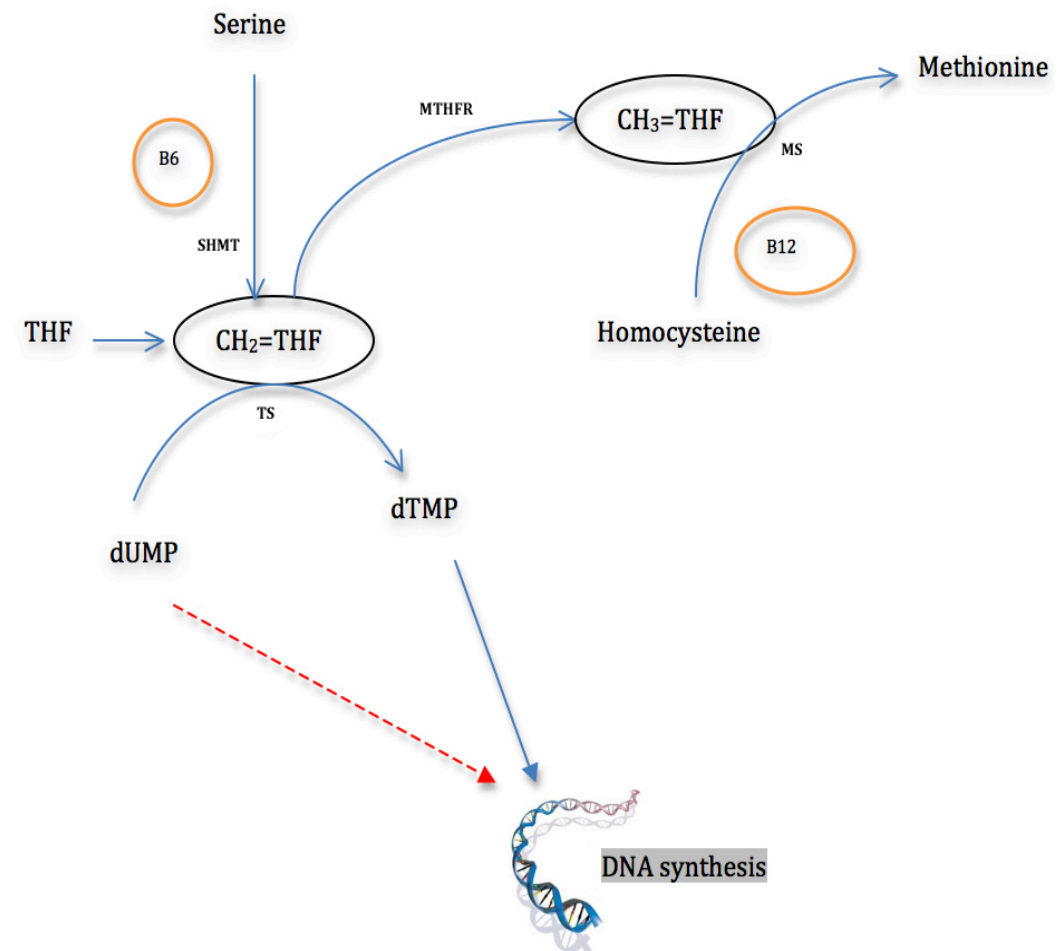

Adapted from Ames \& Wakimoto, 2002. 


\subsubsection{Folate deficiency in rodents}

As mentioned above, folate deficiency can dramatically decrease DNA repair efficiency as it perturbates nucleotide synthesis, thus promoting DNA damage and chromosomal breakage (Sibani et al., 2002; Zhang et al., 2003). Even though folate deficiency has been reported to increase DNA strand breaks (Blount et al., 1997; Kim et al., 2000), direct proof of mutations by sequence analysis is still missing, and therefore the importance of uracil misincorporation into DNA as a mutagenic mechanism still remains unclear (Krokan et al., 2002). Table 1.1.3 summarizes the findings on the effects of isolated folate deficiency on genomic stability in rodents.

As shown in the table below, there is conflicting evidence between studies, revealing that the impact of folate deficiency on genome stability is more complex than previously estimated. 
Table 1.1.3. Impact of folate deficiency on genome stability in rodents.

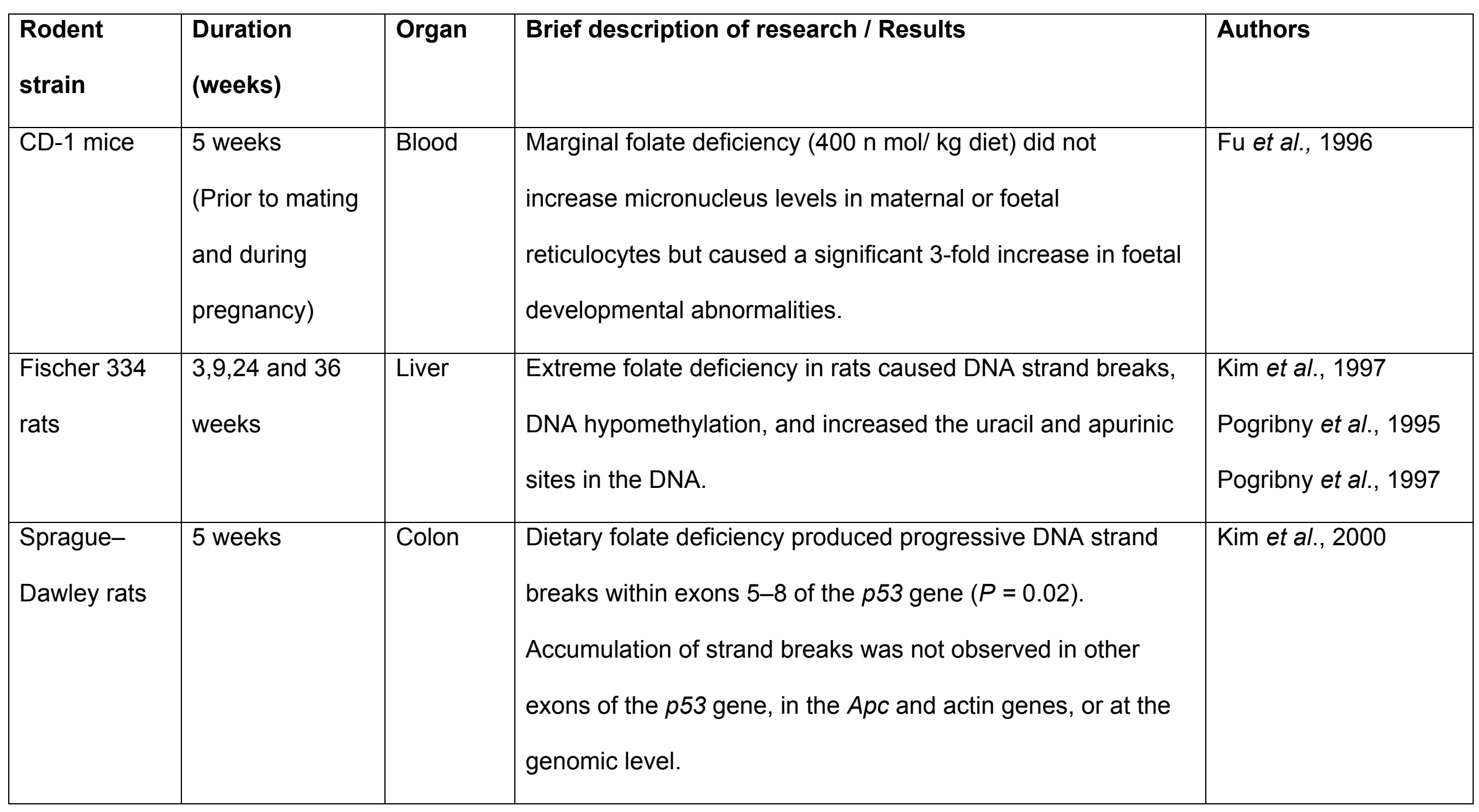




\begin{tabular}{|c|c|c|c|c|}
\hline $\begin{array}{l}\text { Fischer } 334 \\
\text { rats }\end{array}$ & 12 weeks & Brain & $\begin{array}{l}\text { Long-term effects of folate deficient diet caused perturbations } \\
\text { in the levels of one-carbon metabolites along with induction of } \\
\text { oxidative stress, increased expression of DNA repair genes } \\
\text { and oxidative DNA damage in the brain. }\end{array}$ & $\begin{array}{l}\text { Bagnyukova et al., } \\
2008\end{array}$ \\
\hline $\begin{array}{l}\text { Sprague- } \\
\text { Dawley rats }\end{array}$ & 5 weeks & Colon & $\begin{array}{l}\text { Dietary restriction of vitamins involved in folate metabolism, } \\
\text { were found to amplify the loss of genetic integrity of the } p 53 \\
\text { gene that is induced by folate depletion alone. DNA strand } \\
\text { breaks within the hypermutable region of } p 53 \text { were not } \\
\text { significantly induced by mild folate depletion alone. }\end{array}$ & Sohn et al., 2003 \\
\hline
\end{tabular}




\begin{tabular}{|c|c|c|c|c|}
\hline $\begin{array}{l}\text { Fischer } 334 \\
\text { rats }\end{array}$ & $\begin{array}{l}9 \text { weeks or } \\
18 \text { weeks }\end{array}$ & Liver & $\begin{array}{l}\text { Transient DNA damage, global DNA methylation loss, and } \\
\text { hypermethylation of CpG islands in livers of } 9 \text { week treated } \\
\text { rats after turned to sufficient diet. Animals fed the diet for } 18 \\
\text { weeks and returned to sufficient diet were not able to restore } \\
\text { all abnormalities, and only DNA lesions were repaired. }\end{array}$ & Pogribny et al., 2009a \\
\hline CBA mice & 4 weeks & Liver & $\begin{array}{l}\text { A 4-week period of dietary folate depletion caused major } \\
\text { changes in expression of several genes involved in fatty acid } \\
\text { metabolism, DNA synthesis and expression of circadian } \\
\text { genes in the liver. }\end{array}$ & Champier et al., 2012 \\
\hline
\end{tabular}




\subsubsection{Folate deficiency and male fertility}

Even though the impact of folate deficiency has been extensively studied in somatic cells, only a few studies have investigated the effect of folic acid deficiency on germ cells.

Sperm counts are an essential fertility measurement. Decreased sperm counts generally signify impaired spermatogenesis or increased elimination of germ cells during spermatogenesis (Padmanabhan et al., 2008; Swayne et al., 2012b). A study conducted by Wallock and co-authors found a significant association between low seminal plasma folate concentrations, with low sperm density and total sperm count, both in male smokers and nonsmokers, leading to a significant loss of fertility (Wallock et al., 2001). A later study conducted by Boxmeer and co-authors confirmed the previous findings and reported an increase in sperm DNA damage in men (Boxmeer et al., 2009). Folate deficiency was therefore associated with indicators of reduced male fertility. A study conducted by Young and co-authors reported an inverse relationship between total daily folate intake and the frequency of aneuploid sperm in humans (Young et al., 2008).

Animal studies confirm the findings from human population studies.

Padmanabhan and co-authors found a significant decrease in sperm counts and increased sperm DNA damage in male mice treated for 10 weeks with methotrexate, which is an anti-folate chemotherapeutic agent (Padmanabhan et al., 2008; Padmanabhan et al., 2009). A study conducted by Chan and coauthors showed that male mice lacking methylenetetrahydrofolate reductase (MTHFR), a key enzyme in folate metabolism, had a significant reduction in sperm counts (Chan et al., 2010). A study conducted by Swayne and coauthors showed that male mice treated with a folate deficient diet directly 
after weaning for a duration of 15 weeks exhibited a significant decrease in sperm numbers, an increase in germline chromatin damage and DNA mutation (Swayne et al., 2012). Last but not least, a study conducted by Burgoon and co-authors on IRC mice for a duration of 4 weeks on $0 \%$ folic acid diet, revealed a reduction in male reproduction (less offspring per litter), in response to a folate deficient diet and therefore provided a further support to the human data (Burgoon et al., 2002).

These studies demonstrate the importance of folate for sperm health and DNA integrity, suggesting that folate deficiency may be mutagenic to germ cell DNA. Elevated DNA strand breaks caused by extensive uracil misincorporation or hypomethylation are both potential mechanisms, underlying the observed effects. All the above data highlight the importance of adequate folate intake for male fertility and for the prevention of DNA mutations in the germline (Swayne et al., 2012a). 


\subsection{Methyl donors and DNA methylation}

\subsubsection{Epigenetic regulation}

All somatic cells in an organism contain exactly the same genome, even though only a certain subset of genes is activated in each particular type of cell at a given time (Biermann \& Steger, 2007). Gene activity that is transmitted through meiosis and mitosis, but is not accompanied by changes in the coding sequence of DNA, is regulated by the epigenome, which is the total of chromatin modifications that manifest the genetic information (Pogribny \& Beland, 2009). Therefore, the definition for 'epigenetics' is all heritable changes in gene expression involved with DNA and histone modifications, or chromatin proteins that are not the cause of any alterations in the DNA sequence (Su, 2012).

Epigenetic processes include (a) DNA methylation $\left(\mathrm{CH}_{3}\right)$ at deoxycytosine residues within CpG dinucleotide sequences, located within gene promoters ( $\mathrm{CpG}$ islands) in mammals, (b) modification of histones and other DNA-binding proteins via the acetylation, methylation, phosphorylation, and ubiquitinylation of specific amino acid residues within the amino terminus, and (c) gene silencing associated with RNA (Biermann \& Steger, 2007; Pogribny \& Beland, 2009).

All these epigenetic modifications play a key part in the regulation of gene activity without changing the genetic code, and are essential for normal development (regulation of embryonic development, cellular differentiation and organogenesis, as well as imprinting and silencing of large chromosomal domains such as the $\mathrm{X}$ chromosome in females). They are also vital for the accurate maintenance of cellular functions in adult organisms (Su, 2012). 
Even though these epigenetic processes are inherited, they can potentially be reversed by deregulation/modification, causing severe consequences to the phenotype and function of the cell. Epigenetic changes are a usual phenomenon in a plethora of human pathologies (Su, 2012). Numerous human studies have linked many disease states (e.g. Angelman's, Beckwith-Wiedemann and Prader-Willi syndromes) with abnormal epigenetic programming, especially via modifications in the DNA methylation patterns of imprinted genes (Chang et al., 2008).

Furthermore, epigenetic changes have been found to play an important role in the incidence and prevalence of cancer. In the course of malignant transformation, cancer cells can display global changes in the chromatin structure that can affect the whole epigenome, changing the expression of hundreds of genes and perturbing entire metabolic pathways. Consequently, epigenetic deregulation is believed to cause cell damage and severe mutations in DNA coding (Su, 2012).

Diet is one of the environmental factors, which can alter the epigenetic landscape, thus contributing to disease aetiology (Chang et al., 2008). Population studies have revealed that poor choice of diet could account for more than a third of cancer death in the USA (Ames \& Wakimoto, 2002). Since established epigenetic patterns during fetal period can be altered in adulthood by environmental factors including nutrition, diet could severely alter epigenetic patterns in humans and animals (Su, 2012).

It is progressively becoming more obvious that as genetic differences between individuals contribute to individual variation in disease susceptibility, so do the inter-individual differences in their epigenotype. Even though there is a clear understanding on how genetic variation is determined through 
parental inheritance and de novo mutation, the same cannot be said for the factors that contribute to the inter-individual differences in epigenotype as most of them are still elusive. Given that the epigenetic landscape of mammalian cell is not fixed and undergoes massive reprogramming during development, it can potentially be affected by a variety of environmental factors (Waterland, 2006).

\subsubsection{DNA methylation}

As mentioned above, epigenetic processes include DNA methylation. DNA methylation is commonly known as the addition of a methyl group from the universal methyl donor, S-adenosyl-L-methionine (SAM), to the $5^{\prime}$ position of the cytosine pyrimidine ring, resulting in the formation of 5methylcytosine $(5 \mathrm{meC})$. The reaction is catalyzed by DNA methyltransferases (DNMTs) and it leads to the reduction of gene expression. The altered cytosine residues are usually directly next to a guanine nucleotide, resulting in two methylated cytosine residues, sitting diagonally to each other on the opposite DNA strands (Haines et al., 2001; Dodge et al., 2002; Phillips, 2008, Lister et al., 2009; Pogribny et al., 2009a).

Even though DNA methylation at different sequences does exist in eukaryotic embryonic stem cells, in adult somatic tissues DNA methylation is most often found at the cytosine residues at $\mathrm{CpG}$ sequences, and is an essential part of normal development, differentiation, maintenance of cellular homeostasis and function. DNA methylation is associated with numerous key processes including $\mathrm{X}$-chromosome inactivation in females, genomic imprinting, suppression of repetitive DNA elements, regulation of chromatin structure, proper expression of genetic information and carcinogenesis 
(Haines et al., 2001; Dodge et al., 2002; Phillips, 2008, Lister et al., 2009; Pogribny et al., 2009a).

Approximately $70-90 \%$ of the CpG dinucleotides in all mammalian genome are methylated even though the CpG sites are not distributed uniformly across it. Mammalian genomes typically consist of short (usually 4 $\mathrm{kb}$ long) unmethylated domains, entrenched in a matrix of long methylated domains. These CpGs are more often unmethylated than not, and are grouped in clusters called ' $\mathrm{CpG}$ islands' that are found in the 5' regulatory regions of many genes (in promoters and first exons). On the contrary, methylated domains are found mainly in interspersed and tandemly repetitive sequences and exons, except for the first exons. A recent study on 5.549 genes with $\mathrm{CpG}$ island promoters denoted that approximately $4 \%$ of these genes are methylated and silenced under normal conditions (Pogribny et al., 2009a; Pogribny \& Beland, 2009).

A number of key factors such as DNA methyltransferases, DNA demethylase enzymes responsible for histone modification, and the onecarbon metabolism cycle, are vital for the correct preservation of DNA methylation patterns. Changes in any one of these factors could lead to alterations in DNA methylation, including DNA hypermethylation or DNA hypomethylation (Pogribny et al., 2009a). Various studies show that abnormal hypermethylation of gene promoter $\mathrm{CpG}$ islands leads to heritable transcriptional silencing, causing many disease conditions including cancer. On the other hand, hypomethylation has been linked to chromosomal instability and loss of imprinting (Tucker, 2001; Miller \& Sweatt, 2007; DauraOller et al., 2009)

There are two distinct ways by which DNA methylation can impact the 
transcription of genes. The first is that the methylation of DNA can by itself physically obstruct the proteins from binding on to the gene. The second and more important way is that the methylated DNA can be bound by methylCpG-binding domain proteins (MBDs) that have the ability to employ additional proteins to the locus, such as histone deacetylases and other chromatin remodelling proteins that are able to modify histones by forming compact, inactive chromatin, named silent chromatin. Rett syndrome is a neurone-developmental disorder, linked to loss of methyl-CpG-binding protein 2 (MeCP2). This disease state demonstrates the link between DNA methylation and chromatin structure, and reveals its importance in genomic stability. Additionally, a methyl-CpG binding domain protein (MBD2) has been found to arbitrate the transcriptional silencing of hypermethylated genes in cancer (Goll et al., 2006).

A number of human inherited diseases are associated with defective DNA methylation pathways and exhibit abnormal development like the Rett syndrome mentioned above, the ICF (Immunodeficiency, centromere instability and facial anomalies) syndrome and the ATRX (Alpha-thalassemia X-linked mental retardation syndrome) syndrome. Furthermore, aberrant methylation patterns are believed to be involved in tumorigenesis, causing instability, abnormal imprinting and deregulated expression of oncogenes or tumor suppressor genes (Zhu et al., 2006).

Two classes of enzymatic activities carry out DNA methylation, the de novo methylation and maintenance methylation. These two enzymatic activities are distinct and are both essential for the mammalian cell. During the very early stages of mammalian embryogenesis, DNA methylation patterns are largely erased and re-established soon after implantation in a 
wave of de novo methylation. The newly created patterns are then faithfully copied at the end of each replication round, onto the daughter DNA strand. On the other hand, genomic imprints (that mainly depend on DNA methylation) are primarily established in germ cells and are conserved throughout embryogenesis. Consequently, maintenance methylation activity is found in all somatic cells while the maximum de novo methylation activity is found in embryonic cell lines, germ cells, or in post-implantation embryos (Zhu et al., 2006).

\subsubsection{De novo methylation}

In general, DNA methylation is linked with the silencing of genes and the formation of heterochromatin. As both oocyte and sperm are highly specialized and terminally differentiated cells, while the very early embryo is comprised by totipotent blastomeres, it is only logical that a widespread parental-genomic reprogramming must take place. Indeed, for the successful mammalian embryonic development, differential imprinting of both male and female genomes is a necessity. Shortly after the activation of the oocyte by the sperm and the completion of meiosis, the haploid oocyte and sperm chromosomes transform respectively into a maternal and paternal pronucleus. When the protamines from the sperm chromatin are replaced with oocyte cytoplasmic histones, the wide-spread demethylation of the paternal (but not the maternal) DNA takes place. It is interesting to note that the demethylation of paternal DNA occurs in pigs, rats and mice, but not in rabbits and sheep, whose both pronuclei remain highly methylated (Fulka et al., 2004).

DNA methylation is initiated and established by the de novo DNA 
methyltransferase DNMT3 family (DNMT3a and DNMT3b) that are known to set up the DNA methylation patterns early in embryonic development (Goll et al., 2006). Previous studies have shown that expression of the de novo methyltransferases is coordinated by DNMT3L (homologous to the other DNMT3s but with no catalytic activity), lymphoid-specific helicase (Lsh), microRNAs, and piRNAs. It has been also confirmed that DNMT3L aids the de novo methyltransferases by maximizing their ability to bind to DNA and stimulates their activity (Goll et al., 2006). Even though the identification of the enzymes responsible for methylation patterns are now known, the exact molecular mechanisms and important cofactors that aid efficient enzymatic activity to the right sites, remain poorly understood (Zhu et al., 2006).

In an effort to understand the elusive methylation/demethylation mechanism(s) that takes place during human embryogenesis, Fulka and coauthors analysed the pattern of DNA methylation in human zygotes and developing embryos, and discovered that as in other mammalian organisms tested before, the paternal chromatin in the human zygote undergoes active demethylation soon after fertilization. They also found that this process is facilitated by the oocyte cytoplasm as well as by a sperm-derived factor and the male chromatin composition. Additionally, it was revealed that the human zygote represents an intermediate stage between the mouse and the sheep, as the paternal pronucleus in the human zygote does not become completely unmethylated (Fulka et al., 2004).

The authors further state that a decrease of methylation pattern was traceable in the mouse from the two cell embryonic stage to the morula stage, after which de novo methylation was detected in the blastocyst. The inner cell mass (ICM) of the mouse species was intensively methylated, while 
the thymic epithelial (TE) cells were unmethylated. In the contrary IMC in human embryos were found to obtain a weak methylation pattern, whereas the human TE cells were heavy methylated. These distinctive methylation patterns are thought to reflect additional disparities happening a great deal later, at the implantation and placenta formation stages, between the human and the mouse species. The authors concluded that the demethylation processes may be essential for the massive embryonic genome activation that takes place at the two-cell stage in mice and the four-cell stage in humans, respectively (Fulka et al., 2004).

\subsubsection{Maintenance methylation}

Maintenance methylation activity is important for the conservation of DNA methylation, following all cellular DNA replication cycles. DNA methyltransferase1 (DNMT1) is vital for the maintenance of methylation activity, as it aids methylation on the produced daughter strands and prevents passive genome demethylation. In particular, DNMT1 has been known to be responsible for copying the DNA methylation patterns to the daughter strands during DNA replication. The importance of DNMT1 has been thoroughly documented in mouse models in which both copies of DNMT1 were deleted. Those cases leading to embryonic lethality very early in the gestational stage showing that the role of DNMT1 is vital for mammalian cell development (Goll et al., 2006). In addition, a number of studies revealed that disruption of the mouse DNMT1 gene results in genome-wide demethylation and developmental arrest. It is therefore concluded that the role of DNMT1 in propagating parental DNA methylation during replication cannot be substituted by other DNMTs (Li et al., 1992). 
Figure 1.2.1 demonstrates how during DNA replication, DNA methylation is preserved by a complex collaborative interaction of maintenance methyltransferase DNMT1 with the methyl-CpG-binding protein 2 (MeCP2), histone-modifying enzymes, and the UHRF1 protein (Pogribny et al., 2009a). Interestingly, methyltransferase DNMT1 also interacts with the proliferating cell nuclear antigen (PCNA) protein, which creates a topological link to the genome at the replication fork. Therefore a fraction of $\mathrm{CpGs}$ are subjected to maintenance methylation prior to maturation of Okazaki fragments, on the lagging strand. This suggests that DNMT1 may need to be present immediately behind the replication fork in order to endorse efficient methylation of nascent DNA, prior to its packaging into nucleosomes.

However, a large fraction of CpG dinucleotides escapes the first wave of maintenance methylation following the replication fork. Packaging these hemimethylated $\mathrm{CpGs}$ into higher order structures (like nucleosomes and chromatin) could therefore be a way of controlling DNMT1 activity, by preventing their subsequent methylation by DNMT1 (Okuwaki \& Verreault, 2004).

Transcriptional silencing via CpG methylation is a well-documented mechanism, employed on tumor-suppressor genes, in a variety of cancers. Since then, a number of experiments on mouse cells have revealed the role of DNMT1 in the maintenance of methylation and prevention of neoplasia. For example Robert and co-authors reported that selective depletion of DNMT1 in human bladder cancer cells, along with the use of antisense inhibitors, caused demethylation and reactivation of the silenced tumorsuppressor gene CDKN2A (Robert et al., 2002). 


\section{Figure 1.2.1. Methylation processes during DNA replication.}

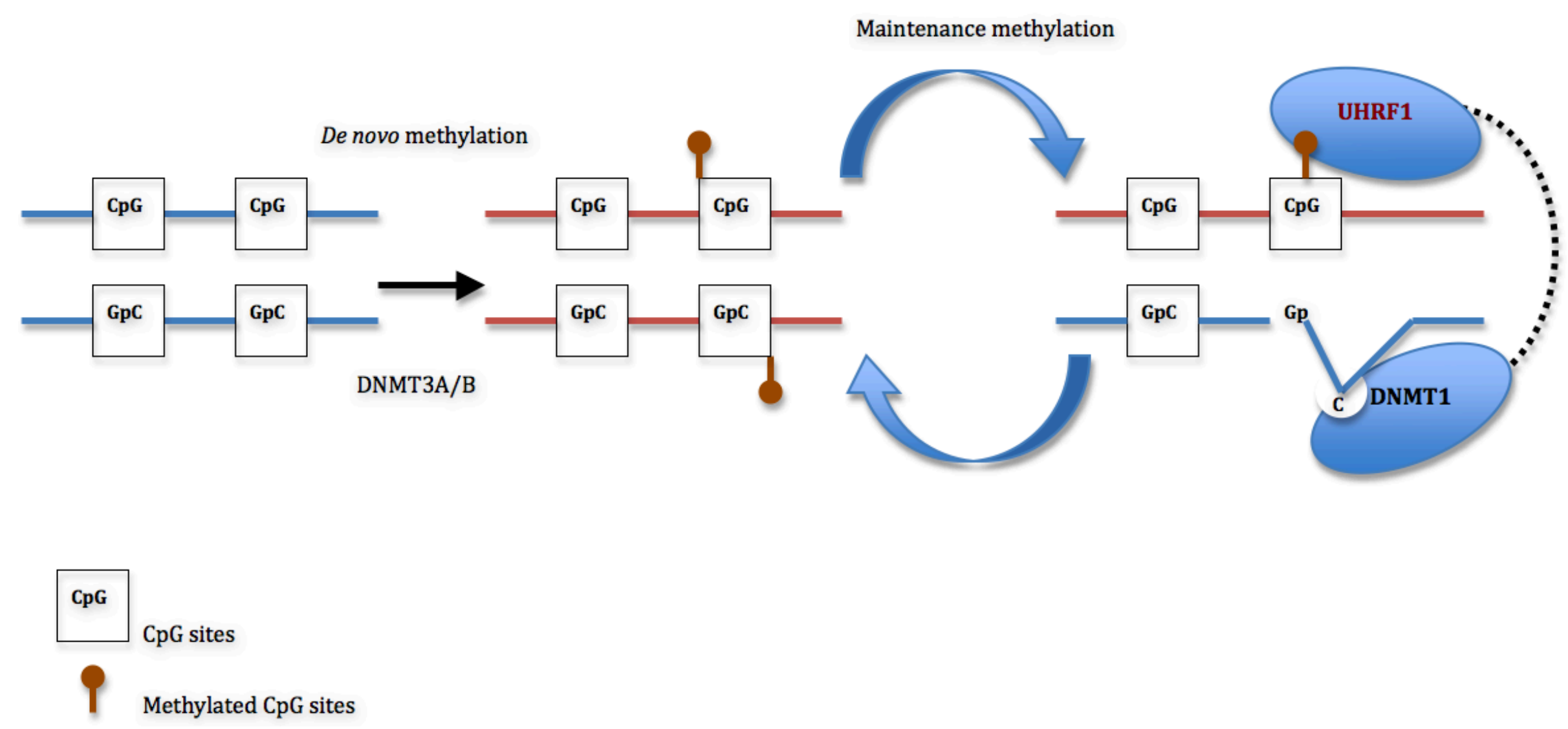

De novo DNMT3A and DNMT3B DNA methyltransferases initiate and establish DNA methylation during embryonic development. After that end for the duration of DNA replication, DNA methylation is maintained by an intricate synchronized work between the two maintenance methyltransferases DNMT1 and UHRF1. The SRA domain of UHRF1 distinguishes the hemimethylated CpG site and recruits DNMNT1, which transports methyl groups to the unmethylated cytosine residue on the newly synthesized DNA strand. Adapted from Pogribny \& Beland, 2009. 
More specifically, Robert and co-authors used both genetic (antisense and siRNA) and pharmacologic (5-aza-2'-deoxycytidine) inhibitors of DNA methyltransferases when investigating the contribution of the DNMT isotopes to cancer-cell methylation, and discovered that selective depletion of DNMT1 resulted in (a) lower cellular maintenance methyltransferase activity, (b) global and gene-specific demethylation and (c) re-expression of tumorsuppressor genes in human cancer cells. More specifically, depletion of DNMT1 was found to distinctly increase the ability of 5-aza-2'-deoxycytidine to reactivate silenced tumor-suppressor genes, demonstrating that inhibition of DNMT1 function is the primary way by which 5-aza-2'-deoxycytidine reactivates genes. The recorded results clearly show that DNMT1 is essential and adequate to preserve global methylation and abnormal $\mathrm{CpG}$ island methylation in human cancer cells (Robert et al., 2002).

\subsubsection{Mechanisms of DNA demethylation}

There are two distinct mechanisms by which demethylation is known to occur. The first is the 'Active DNA demethylation' and the second is the passive or 'Replication Coupled demethylation' process.

\subsection{Active DNA demethylation}

The chemistry necessary to actively demethylate 5-methylcytosine (5$\mathrm{meC}$ ) is challenging, and requiries the disruption of a $\mathrm{C}-\mathrm{C}$ bond. Some possible mechanisms have been proposed for active DNA demethylation and are presented here: (a) base excision repair initiated by 5-meC DNA Glycosylases (b) 5-meC deamination coupled with G/T Mismatch Repair (c) Oxidative Demethylation. 
The first mechanism involves base excision repair, which is initiated by DNA glycosylases (Jost, 1993; Jost et al., 1995). The DNA glycosylases cleave the glycosidic bond between the 5-meC base and the deoxyribose, creating an abasic site or AP site. An AP endonuclease then removes the deoxyribose at the AP site and finally, the gap is filled by DNA polymerase and DNA ligase. This base excision repair pathway therefore results in the removal of methylated cytosine, and its replacement by an unmethylated cytosine (Wolffe et al., 1999; Zhu, 2009).

The second mechanism of active DNA demethylation is initiated through the enzymatic deamination of $5-\mathrm{meC}$ to $\mathrm{T}$, coupled with $\mathrm{G} / \mathrm{T}$ mismatch repair by DNA glycosylases (Morgan et al., 2004). It was recently proposed that the de novo DNA methyltransferases DNMT3a and DNMT3b are able to convert 5-meC to $T$ through deamination. The resulting $T$ is then removed by a G/T mismatch base excision repair pathway (Metivier et al., 2008). Previous studies have shown that DNMT3a (Li et al., 2007) and DNMT3b (Boland et al., 2008) also interact with the G/T mismatch repair DNA glycosylases.

These interactions may indeed reflect the potential coupling between the 5-meC deamination activities of DNMT3s and the G/T mismatch DNA glycosylase for the achieving of DNA demethylation (Metivier et al., 2008). On the other hand, these interactions may simply allow the mismatch DNA glycosylase to be recruited to the methylated DNA for the prevention of $\mathrm{C}$ to T mutations caused by spontaneous deamination of 5-meC (Li et al., 2007; Boland et al., 2008).

Last but not least, it is possible that active DNA demethylation may also be achieved by an oxidative demethylation mechanism. It has been recorded that the Alkb family of DNA repair enzymes are able to remove the methyl 
group from 1-methyladenine and 3-methylcytosine by oxidative demethylation, via the employment of $\mathrm{Fe}(\mathrm{II}) / \alpha-$ ketoglutarate-dependent oxygenases as they drive the catalysis of the release of the methyl moiety as formaldehyde (Falnes et al., 2002; Trewick et al., 2002).

Another possible active DNA demethylation process driven by the jumonji C (JmjC) domain-containing proteins has been recorded to oxidative demethylate histone H3 lysine 9 or histone H3 lysine 36 (Tsukuda et al., 2006; Yamane et al., 2006). The JmjC domain histone demethylases also use the $\mathrm{Fe}(\mathrm{II}) / \alpha$-ketoglutarate cofactors, and the methyl moiety is released as formaldehyde during the reaction. Additionally, demethylation of methylated histone H3 K4 by lysine-specific demethylase 1 (LSD1) involves an oxidative reaction, which uses flavin adenine dinucleotide (FAD) as a cofactor while releasing formaldehyde as a by-product (Shi et al., 2004).

All mechanisms mentioned above, (hydrolysis as well as oxidative demethylation mechanisms) are direct, one-step reactions catalyzed by a single enzyme. The base and nucleotide excision repair pathways could also be considered as direct mechanisms, but they require multi-step reactions and employ numerous enzymes. In contrast, the mechanisms coupling deamination of 5-meC with base excision repair are indirect mechanisms and require multi-step reactions (Zhu, 2009).

\subsection{Replication-Coupled Demethylation}

Rescentt studies have revealed that DNA replication could also contribute to the elimination of 5-methylcytosine from DNA. The role of DNMT1 methyltransferase in the replication fork and the rapid maintenance methylation of $\mathrm{CpG}$ dinucleotides has previously been described (Figure 


\subsection{1).}

It has been previously reported that chromatin structures as well as nucleoprotein complexes linked to transcriptional activity can interfere/ obstract the DNMT1 activity and therefore cause a progressive reduction of DNA methylation levels, with each passing cell division. As illustrated in Figure 1.2.2, during replication, both strands of a symmetrically methylated DNA will be segregated to daughter chromatids. At the end of this event both daughter chromatids will contain hemimethylated DNA, and under normal circumstances, DNMT1 restores symmetric methylation.

The first mechanism of 'passive' or replication-coupled DNA demethylation dictates that it is possible for sequence-specific transacting factors to bind over the sites of DNA methylation and therefore prevent access of the methyltransferase enzyme to these sites, leading to the progressive demethylation of DNA, potentially in a sequence specific manner.

With respect to a more global genome demethylation, a second mechanism of replication-coupled demethylation could be activated when specific histone modifications like acetylation might exercise a so called 'teflon effect' where it will repel/prevent the efficient access of the DNMT1 methyltransferase enzyme to the newly synthesised strand, leading to the loss of methylation.

The two models predict that DNA replication is an integral component of demethylation. (Wolffe et al., 1999). 
Figure 1.2.2. Replication-coupled DNA demethylation.

\section{A. Norma}
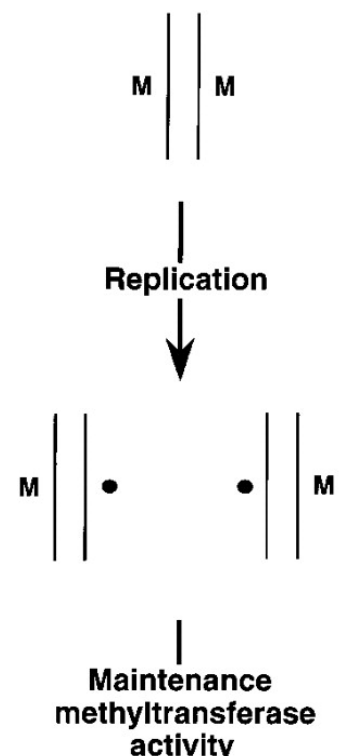

$\downarrow$
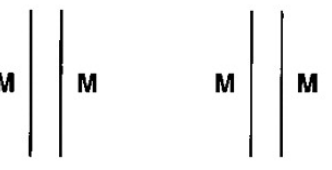

B. Nucleoprotein occlusion
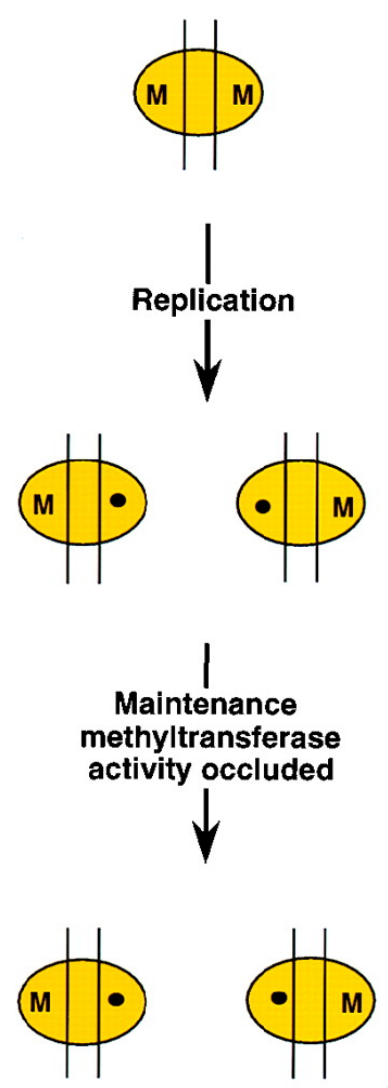

\section{Acetylated histone aversion}
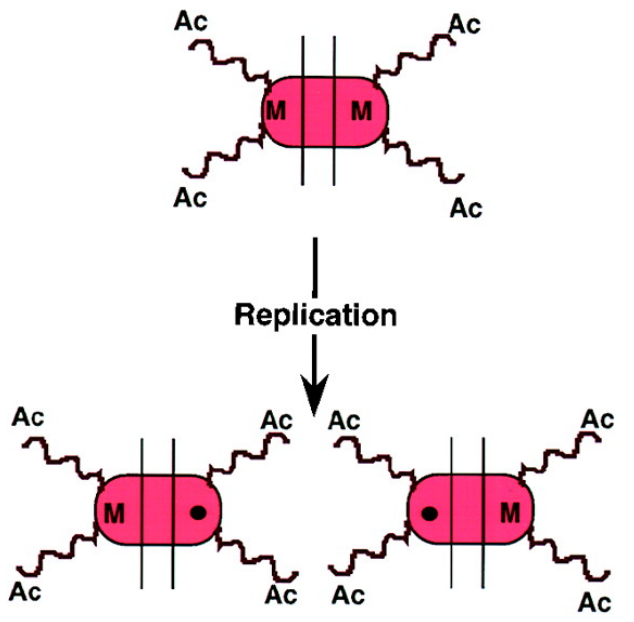

\section{Maintenance \\ methyltransferase}

activity repelled

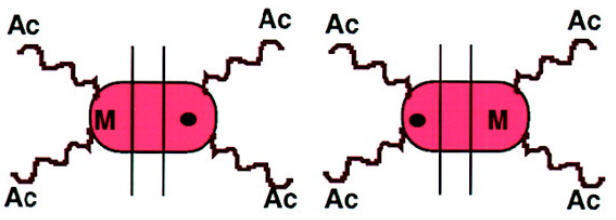

Part (a): Normal way by which each strand (methylated CpG dinucleotide represented with an 'M') will be segregated after replication to the daughter chromatids, leaving one strand of DNA methylated $(\mathrm{M})$ and the other not $(\bullet)$. DNMT1 will then restore the pattern of symmetrical methylation. Part (b): Regulatory nucleoprotein complexes occluding DNMT1 activity can lead to loss of methylation. Part (c): Histone acetylation might repel or inhibit DNMT1, leading to the loss of methylation. Figure taken from Wolffe et al., 1999. 


\subsubsection{Methionine importance to DNA methylation}

Methionine is a sulfur containing amino acid, used in protein formation and it is the preparatory material for multiple biochemical molecules, including the two amino acids cystein and cystine. Methionine promotes production of lecithin in the liver, which helps to the reduction of cholesterol levels in the blood, reduction of liver fat and kidney protection. It also regulates ammonia levels in the urine and aids heavy metal excretion from the body. Additionally, methionine is one of the two amino acids (the other being tryptophan), encoded by the codon (AUG) that is the 'start' signal for ribosomes to initiate protein translation from mRNA (Ellis, 2008).

Another essential role of methionine is its conversion to Sadenosylmethionine (SAM), by the enzyme methionine adenosyltransferase (MAT). SAM is the most important methyl group donor for the majority of biological reactions such as DNA methylation, as well as methylation of lysine and arginine residues in histones (Chiang et al., 1996). SAM is therefore vital for the determination of gene expression and prevention of chromosomal breakage, particularly in regions like the centromeric and fragile sites (Eto et al., 1986; Beetstra et al., 2005). Additionally, methylation by SAM is essential for protein stabilization (e.g myelin) and is implicated in the synthesis of many molecules including melatonin, norepinephrine, and carnitine. SAM is also needed in the formation of polyamines and in the metabolism of serotonin and niacinamide (Caudill et al., 2001; Miller, 2003).

As seen below in Figure 1.2.3, following donation of its methyl group and regardless of the methyl-group acceptor involved, SAM is converted to $\mathrm{SAH}$, which is converted to homocysteine after losing its adenosine by a reversible reaction catalyzed by the enzyme SAH hydrolase. Homocysteine 
can then be recycled to methionine by two pathways; (a) by the enzymatic action of methionine synthetase and the taking up of a methyl group taken from methylcobalamin, or (b) by the enzymatic action of betainehomocysteine methyltransferase, and the taking up of a methyl group taken from betaine, or it can be converted to amino acids cysteine and taurine by the enzymatic action of cystathione betasynthase (Caudill et al., 2001; Miller, 2003).

Figure 1.2.3. SAM cycle pathway.

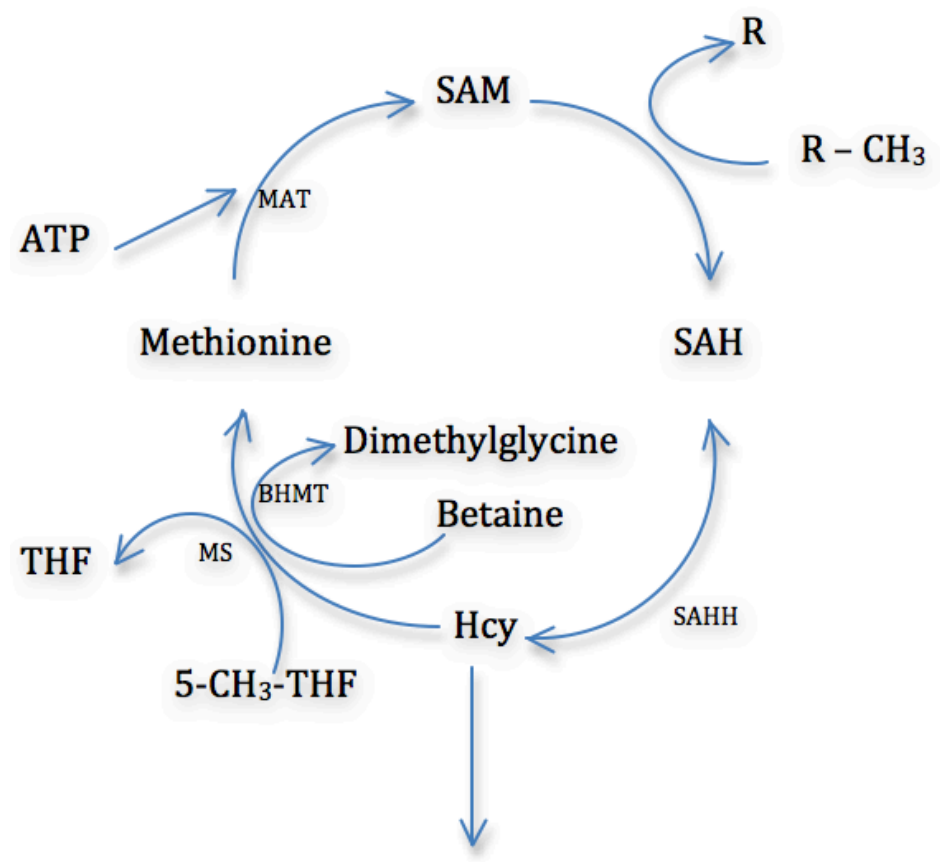

Cystathionine

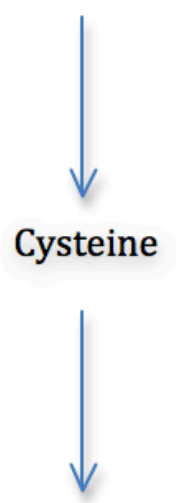

Glutathione

Adapted from Caudill et al., 2001. 
As mentioned above, methionine metabolism is essential for several bodily functions and biochemical processes. As the human body does not synthesize methionine, it is important to be taken up by food consumption, and either dietary deprivation, or dietary deficiencies caused by genetic enzyme polymorphisms, can be harmful to normal metabolism. More specifically, a dietary deficiency of either folic acid (which acts as a methyl donor for the remethylation of homocysteine), vitamin $B_{12}$ or vitamin $B_{6}$ which are all cofactors in the methionine and homocysteine metabolism can lead to hyperhomocysteinemia, a medical condition characterized by a considerable rise in the level of homocysteine in the blood leading to artery defects, blood clots, heart attack and strokes (Caudill et al., 2001; Miller, 2003).

Last but not least, the ratio of SAH to SAM is often used as a marker for the cell's methylation capacity, as decrease in this ratio predicts the reduction of methylation potential (Sibani et al., 2002).

\subsubsection{Choline importance to DNA methylation}

Choline is an essential chemical that the body can produce in small quantities, even though its main source comes from diet. Some amounts of choline can be synthesized from methionine through the methylation of phosphatidylethanolamine by phosphatidylethanolamine $\mathrm{N}$-methyltransferase (PEMT) using S-adenosylmethionine as the methyl donor. Choline is similar to most B vitamins and is part of many major phospholipids such as phosphatidylcholine, which is important to a variety of cell functions raging from the structure of the cell bilayer membrane to the control of cell growth and gene expression (Davison et al., 2008). Choline is also the metabolic precursor of the acetylcholine neurotransmitter (Blusztajn, 1998). It has the 
ability to cause behavioural, neuroanatomical, and electrophysiological alterations, as recorded in studies monitoring postnatal life following prenatal supplementation and deficiency. An increasing number of studies now suggest, that choline's vital importance could lie in its role as a methyl group donor.

Choline is the major betaine precursor used by the kidneys to preserve water balance. It is oxidized to betaine via the enzymatic catalysis of the mitochondrial choline dehydrogenase (located on the matrix of the mitochondrial inner membrane) and by doing so results to the formation of two ATP molecules and becomes a donor of methyl groups for enzymatic methylation (Finkelstein et al., 1983). Betaine is later on used for the conversion of homocysteine to methionine, and subsequently to Sadenosylmethionine (SAM) (Finkelstein et al., 1983).

In recent years, numerous animal (Meck \& Williams, 2003; McCann et al., 2006; Zeisel, 2006) and human studies (Shaw et al., 2004) have established the importance of choline during pregnancy and its vital role to the fetus development, especially for the development and function of the central nervous system. In rat models utilized for the evaluation of choline consumption during pregnancy and its effect to the offspring, pregnant rats were subjected to diets of varying choline content for a period of only one week during the second half of the gestation. The data revealed that choline deficiency caused impairment of specific memory tasks, modification of fetal hippocampal cell proliferation, apoptosis and differentiation (Meck \& Williams, 2003). On the other hand, choline supplementation was found to alter the patterns of hippocampal and cerebral cortical gene expression, both during postnatal development and in adulthood (Mellott et al., 2007). Additionally, 
the results of another study revealed an increased adult hippocampal neurogenesis, leading to improvement of memory and attention in the offspring (Glenn et al., 2008). Also two other studies found a link between adequate choline consumption and prevention of age related memory decline (McCann et al., 2006; Meck et al., 2008).

Studies on adult mice revealed that high choline intake increased the size of cholinergic neurons in the basal forebrain (Williams et al., 1998), enhanced acetylcholine storage and release (Meck et al., 2008), and raised the concentrations of numerous neurotrophins including NGF, BDNF, and NT3 (Sandstrom et al., 2002; Glenn et al., 2007; Glenn et al., 2008). Moreover, high choline intake was found to lower the stimulation threshold for induction of long-term potentiation (Pyapali et al., 1998; Jones et al., 1999), to increase depolarization-induced mitogen-activated protein kinase (MAPK) activation, and CAMP-response element binding protein (CREB) phosphorylation, in the postnatal hippocampus (Mellott et al., 2004).

Even though the entire mechanism of choline's action, especially how it interacts with other nutrients is not completely understood, it is now known that the metabolic pathways of both choline, methionine and folate are very closely linked, since their metabolic pathways meet during the methionine synthesis from homocysteine (Davison et al., 2008). In a number of in vivo studies the role of dietary choline and methionine and their impact on global and candidate gene DNA methylation, have been analyzed. Table 1.2.1 summarizes these findings. 
Table 1.2.1. Impact of choline and methionine deficiency on DNA methylation.

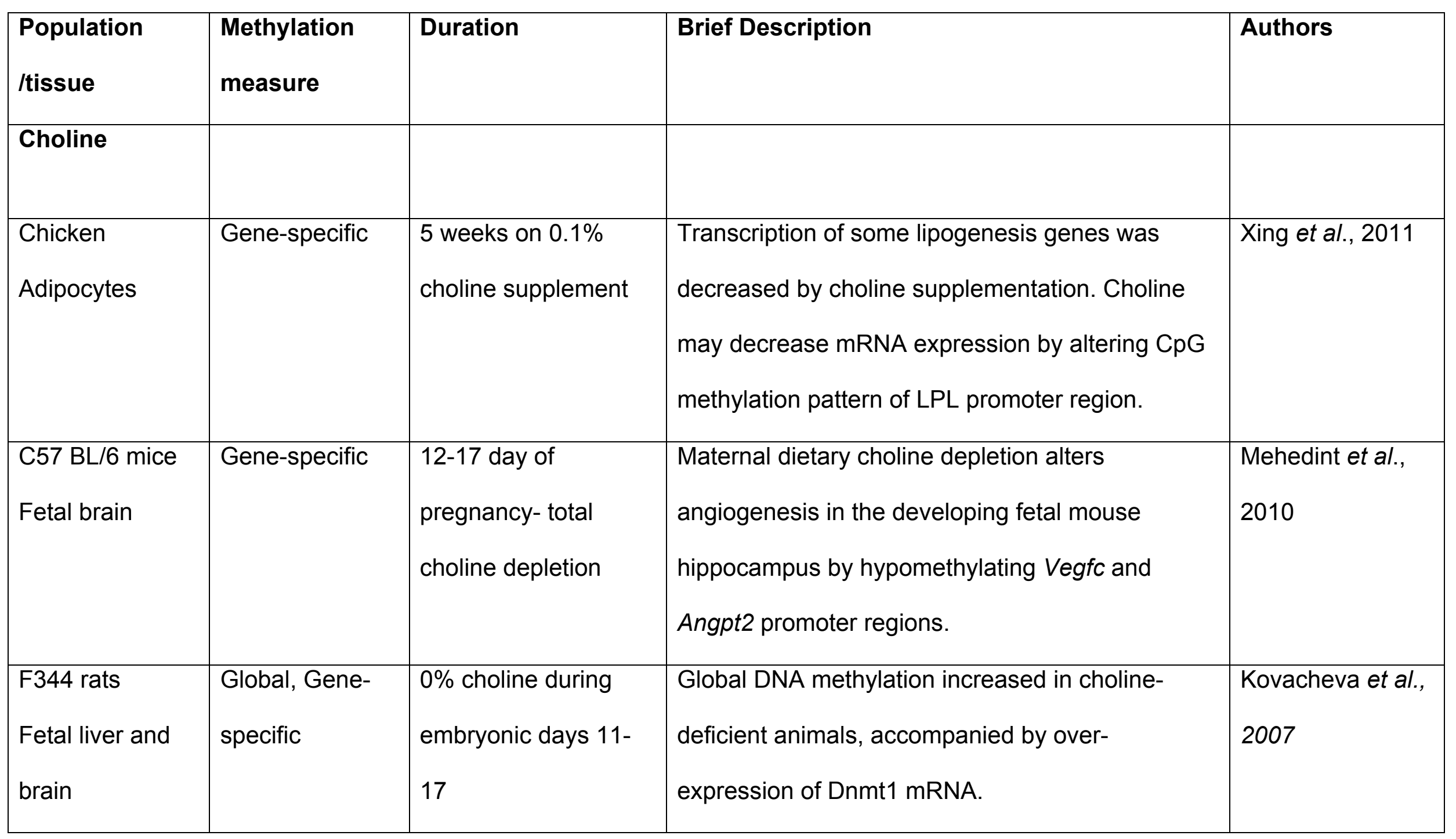




\begin{tabular}{|c|c|c|c|c|}
\hline $\begin{array}{l}\text { C57 BL/6 mice } \\
\text { Fetal brain }\end{array}$ & $\begin{array}{l}\text { Global, Gene- } \\
\text { specific }\end{array}$ & $\begin{array}{l}0 \% \text { choline during } \\
\text { embryonic days } 12- \\
17\end{array}$ & $\begin{array}{l}\text { Choline deficiency induced changes in gene } \\
\text { methylation that can mediate the expression of } \\
\text { cell cycle regulation and therefore alter brain } \\
\text { development. }\end{array}$ & $\begin{array}{l}\text { Niculescu et al., } \\
2006\end{array}$ \\
\hline $\begin{array}{l}\text { Wistar rats } \\
\text { Adult kidneys }\end{array}$ & Gene-specific & $\begin{array}{l}2 \% \text { methionine } \\
\text { supplementation for a } \\
\text { duration of } 6 \text { weeks }\end{array}$ & $\begin{array}{l}\text { Supplementation increased the renal } \\
\text { concentration of SAH without changing the } \\
\text { SAM/SAH ratio. This study was inconclusive on } \\
\text { the effects of genomic stability and DNA } \\
\text { methylation. }\end{array}$ & $\begin{array}{l}\text { Amaral et al., } \\
2011\end{array}$ \\
\hline $\begin{array}{l}\text { Humans } \\
\text { Leukocytes }\end{array}$ & Gene-specific & $\begin{array}{l}\text { Population study } \\
162 \text { male lung cancer } \\
\text { cases and controls }\end{array}$ & $\begin{array}{l}\text { Methionine was associated with decreased } \\
\text { methylation levels of RASSF1A tumor suppressor } \\
\text { gene. }\end{array}$ & Vineis et al., 2011 \\
\hline
\end{tabular}

Adapted from Anderson et al., 2012. 


\subsubsection{Folate importance to DNA methylation}

As mentioned in Section 1.1.4, folate is reduced to DHF and then to THF, serving as a single carbon donor in the form of 5-methylTHF (Figure 1.1.2). The 5-methylTHF is used in the one-carbon metabolism cycle by donating its methyl group to homocysteine, converting it to methionine (Eto et al., 1986; Beetstra et al., 2005) and by that, replenishing cellular SAM (Caudill et al., 2001; Fenech, 2001; Anderson et al., 2012).

Depletion of folate leads to a cascade of events, starting with low production and low concentrations of methionine, reduction of SAM and compromised SAM-dependent methylation reactions, leading to DNA hypomethylation. Reduction or inhibition of SAM decreases the activity of methylenetetrahydrofolate reductase (MTHFR), resulting in the irreversible conversion of 5,10-methylenetetrahydrofolate to 5-methyltetrahydrofolate. This favours the increase in the dUMP pool and promotes an even bigger uracil incorporation into DNA. Furthermore, the reduction of SAM also results in an increase in cellular concentrations of SAH by promoting homocysteine accumulation via reversal of SAH hydrolase (Caudill et al., 2001; Fenech, 2001). Many current studies use a candidate gene-specific approach to examine the effects of folate on DNA methylation. The selected genomic loci are usually linked with a disease outcome of interest (in preventative or therapeutic strategies) such as cancer or diabetes, and these studies usually explore folate's role in methylation of target tissue types (Anderson et al., 2012). Table 1.2.2 summarizes the findings on the link between dietary folate and epidemiological DNA methylation research in different population groups. 
Table 1.2.2. Impact of folate deficiency on DNA methylation in humans.

\begin{tabular}{|c|c|c|c|}
\hline Population/tissue & $\begin{array}{l}\text { Methylation } \\
\text { measure }\end{array}$ & Brief description & Authors \\
\hline $\begin{array}{l}\text { Primary breast tumors } \\
\text { in a small cohort of } \\
\text { women }\end{array}$ & Epigenome-wide & $\begin{array}{l}\text { Folate deficiency caused decrease in global genome } \\
\text { methylation patterns coupled with gene } \\
\text { upregulation. }\end{array}$ & Christensen et al., 2011 \\
\hline $\begin{array}{l}\text { Lung cancer cases and } \\
\text { controls }\end{array}$ & Gene-specific & $\begin{array}{l}\text { Folate associated with increased levels of RASSF1A } \\
\text { and MTHFR gene expression in leukocytes }\end{array}$ & Vineis et al., 2011 \\
\hline
\end{tabular}




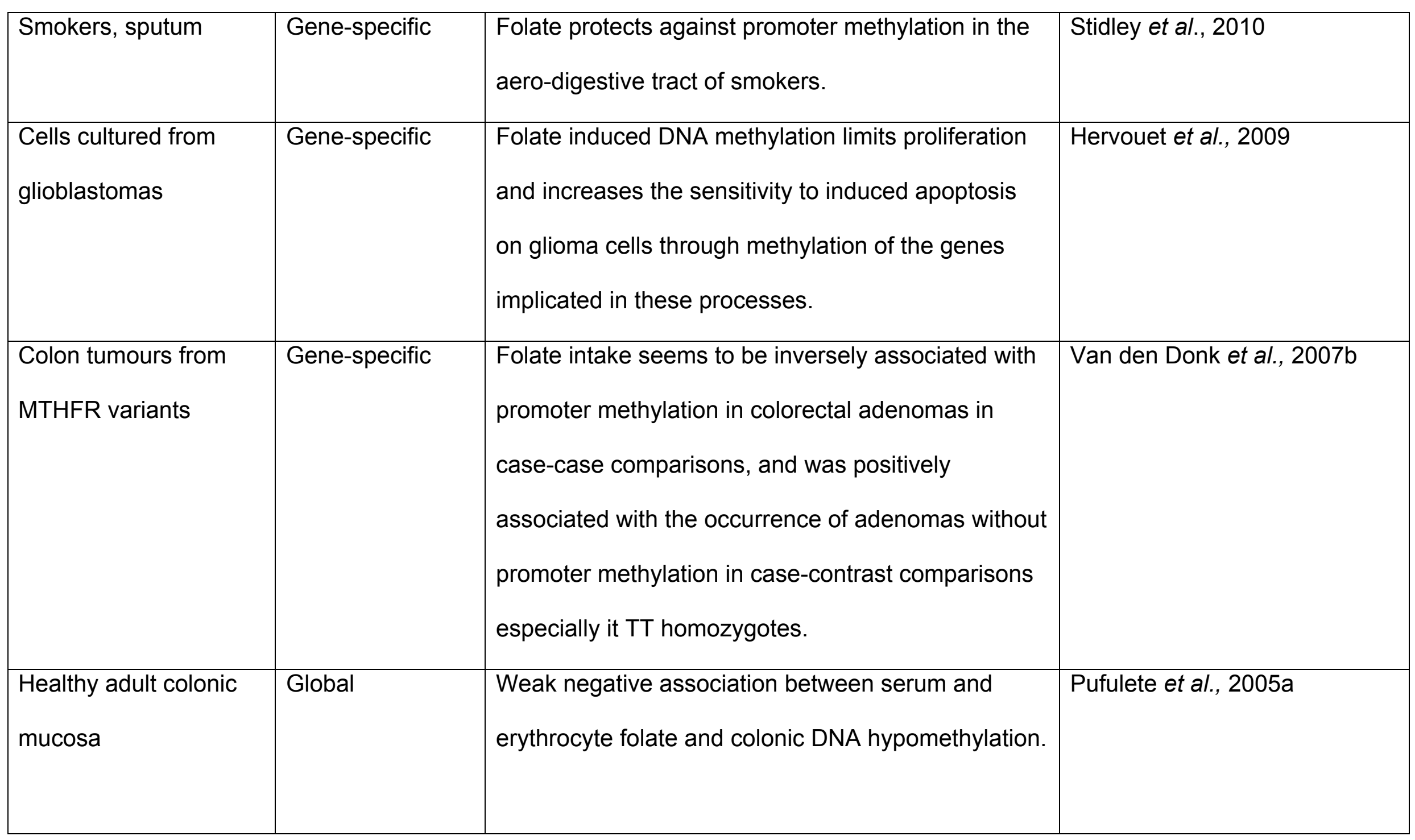




\begin{tabular}{|c|c|c|c|}
\hline $\begin{array}{l}\text { Adults with CRC, } \\
\text { leukocytes and colonic } \\
\text { mucosa }\end{array}$ & Global & $\begin{array}{l}\text { DNA hypomethylation can be reversed by } \\
\text { physiological intake of folic acid. }\end{array}$ & Pufulete et al., 2005b \\
\hline $\begin{array}{l}\text { Adult women, } \\
\text { leukocytes }\end{array}$ & Global & $\begin{array}{l}\text { Moderate folate depletion in young women may } \\
\text { cause a decrease of genes involved in CRC } \\
\text { carcinogenesis. }\end{array}$ & Shelnutt et al., 2004 \\
\hline Colon tumors & Gene-specific & $\begin{array}{l}\text { The prevalence of promoter hypermethylation was } \\
\text { higher in CRCs derived from patients with low } \\
\text { folate/high alcohol intake, which suggests that folate } \\
\text { and alcohol intake may be associated with changes } \\
\text { in promoter hypermethylation in CRC. }\end{array}$ & Van Engeland et al., 2003 \\
\hline $\begin{array}{l}\text { Postmenopausal } \\
\text { women, leukocytes }\end{array}$ & Global & $\begin{array}{l}\text { Blood samples of a small cohort study of } 33 \text { women } \\
\text { aged } 63 \text { and above, showed that low levels of folate } \\
\text { cause global DNA hypomethylation in the } \\
\text { leukocytes. }\end{array}$ & Rampersaud et al., 2000 \\
\hline
\end{tabular}

Adapted from Anderson et al., 2012. 
The data on folate's role in methylation status of selected genes known to alter disease susceptibility in humans is complicated and conflicting. Van de Donk and co-authors reported that folate intake did not have any significant effect on colorectal cancer (CRC) associated genespecific methylation of white blood cells (Van den Donk et al., 2007a). Similarly, a cohort study in Netherlands showed that low-folate intake combined with high alcohol consumption resulted in non-significant changes in gene promoter methylation in blood of CRC patients (Van Engeland et al., 2003). A study conducted by Stidley and co-authors examined the pattern of DNA methylation in 8 genes found to hypermethylate in lung cancer patients. The study reported that 5 out of those 8 genes had a significant decrease in methylation status after treatment with increased serum folate (Stidley et al., 2010).

On the other hand, Vineis and co-authors reported a positive correlation between serum folate concentrations and promoter methylation in the RASSF1A tumor suppressor gene. Increased expression of this gene has been linked to numerous diseases, such as breast, lung and colon cancer. The reported increase in promoter methylation may repress the RASSF1A gene expression, thus consequently decrease cancer risk (Vineis et al., 2011). The data combined suggest that folate plays an important role in DNA methylation and even though the link is still unclear, folate could be associated with protection against some cancers.

Table 1.2.3 summarises the findings on the effects of isolated folate deficiency on DNA methylation in rodents. 
Table 1.2.3 Summary of the effects of isolated folate deficiency on DNA methylation in rodents.

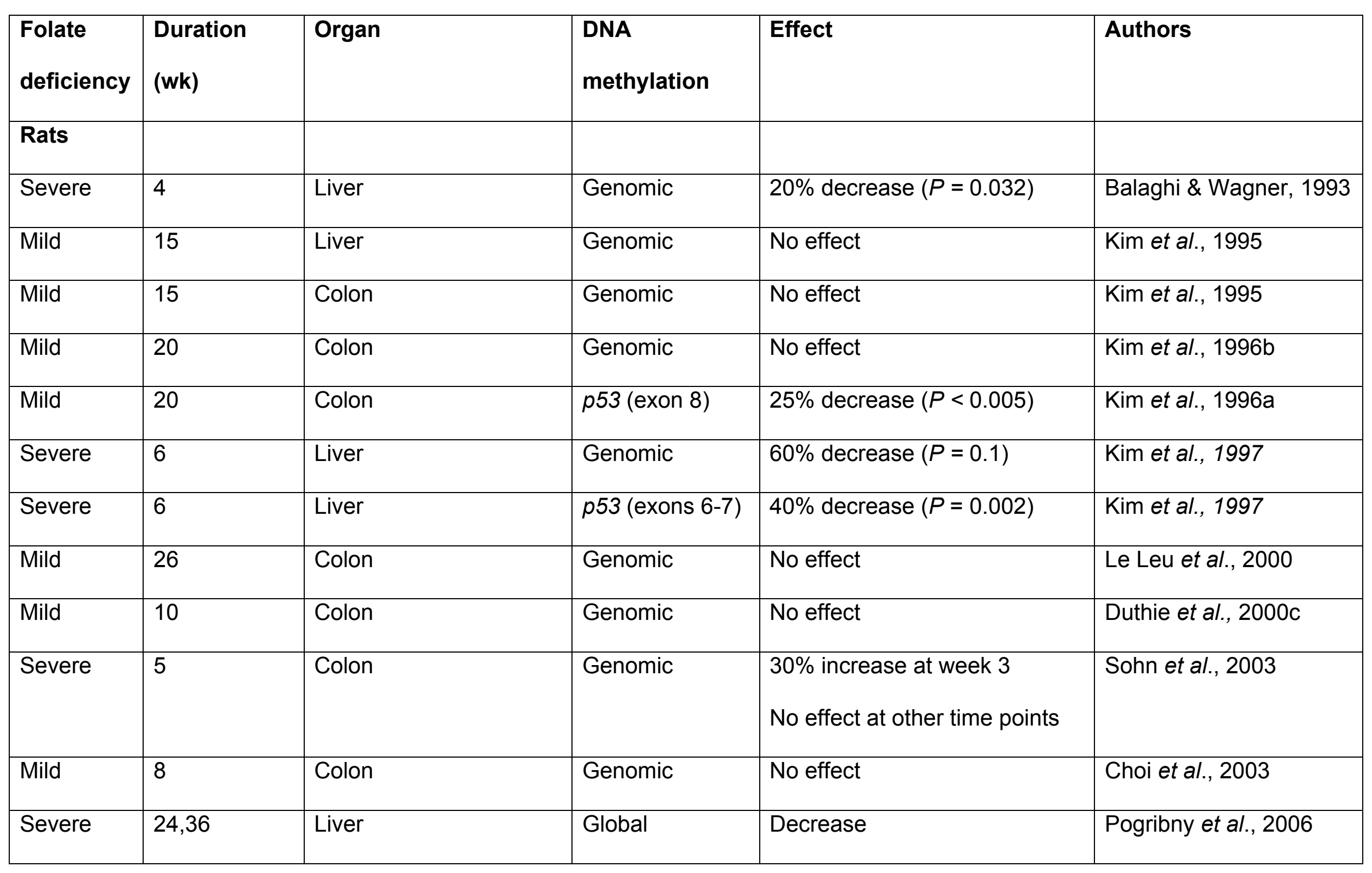




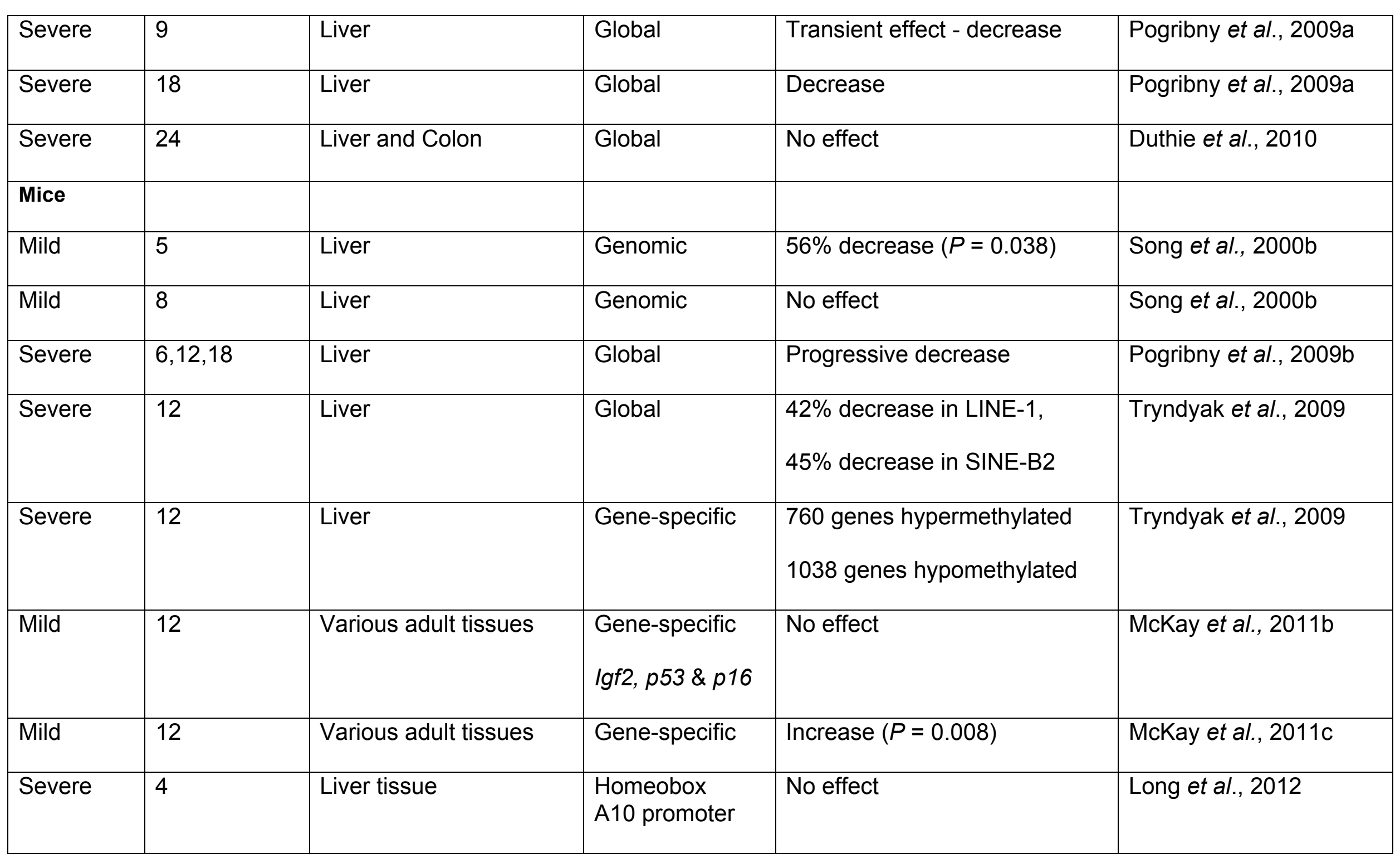

Adapted from Kim, 2004. 
As the table shows, there is conflicting evidence between studies. Some studies show that the impact of folate deficiency ranges between $60 \%$ decreases in the methylation status of specific DNA regions, to no recorded changes. Other studies reported significant methylation effects on genomic DNA in specific tissues where others did not, while one recorded a transient increase in methylation status. The conflicting evidence suggests that the mechanisms associated with micronutrient influence on DNA methylation, is once again, more complex than previously estimated (Kim, 2003; Kim, 2004; Anderson et al., 2012).

\subsubsection{DNA methylation and cancer}

Since DNA methylation has an essential role in gene expression and cell differentiation, it appears obvious that methylation errors can create several damaging and harmful consequences, including a wide range of diseases. In fact, medical scientists are now examining the links between methylation abnormalities and diseases such as cancer, lupus, muscular dystrophy, and a wide variety of birth defects that show close relation with defective imprinting mechanisms (Robertson, 2005).

An increasing amount of scientific papers on DNA methylation and disease focus on cancer and tumor suppressor genes. It has been recorded that tumor suppressor genes are usually silenced in cancer cells due to hypermethylation. On the other hand, the genomes of cancerous cells have been found to be globally hypomethylated, except of some cases of hypermethylated genes involved in the regulation of cell cycle, tumor cell invasion and DNA repair. Interestingly, in some cancers such as colon 
cancer, hypermethylation can be detected at a very early stage and thus can be used as a biomarker for this disease (Phillips, 2008).

\subsubsection{DNA hypomethylation}

DNA hypomethylation refers to the relative decrease from the cell's 'normal' methylation level; a very important DNA methylation state altogether (Dunn, 2003). It is well documented that a disease state can by itself stimulate DNA hypomethylation, but vice-versa reduction in DNA methylation can play a critical role in pathological predisposition and disease development. How DNA hypomethylation occurs is yet unknown but it is believed that more than one mechanisms are responsible for it (Dunn, 2003; Wilson et al., 2007; Pogribny \& Beland, 2009).

An increasing amount of data clearly indicate, the normal function of DNA methyltransferases (DNMTs) is vital for the preservation of accurate DNA methylation (Li et al., 1992) and reduction in the expression of one or more DNMTs has been linked to noticeable reduction in global methylation levels (Jamaluddin et al., 2007). It is important to note that only a $10 \%$ reduction of the Dnmt1 expression is enough to cause substantial hypomethylation of repetitive sequences in the centromere and hypomethylation of endogenous retroviral intracesternal ' $A$ ' particle (IAP) repetitive sequences, in mice (Pogribny \& Beland 2009).

Moreover, data have shown that compromising DNMT function after the inhibition of its activity with demethylating agents, such as 5-aza-20deoxycytidine-homocysteine (Creusot et al., 1982), or its metabolite SAH, has a consequence of a prompt DNA global demethylation. It should be noted that exposure to some environmental factors, including arsenic (Seitz 
\& Stickel, 2007), tobacco (Liu et al., 2007) and alcohol (Seitz \& Stickel, 2007) can inhibit the expression or activity of DNA methyltransferases, resulting in DNA demethylation (Pogribny \& Beland, 2009).

There are two groups of risk factors that can affect the normal functioning of the one-carbon metabolic pathway and therefore alter the pattern of DNA methylation. The first group of risk factors includes all the non-changeable 'genetic' risk factors like genetic variations, that incorporate functional polymorphisms in genes, which encode for enzymes involved in the cellular one-carbon metabolism, that have been linked to abnormal DNA methylation statuses (Niculescu \& Zeisel, 2002; Ulrey et al., 2005).

The second group of risk factors includes all the potential 'adjustable' factors such as all the important micronutrients involved in the metabolism of methyl groups, including methionine, choline, folic acid, and vitamin $B_{12}$ (Oommen et al., 2005). Numerous studies on mice and rats have verified that inadequate intake of these micronutrients for a long period of time results in a significant loss of cytosine methylation in the liver (Wainfan \& Poirier, 1992; Christman, 1995; Zhao et al., 1997).

Furthermore, genome integrity is itself a critical factor for the preservation of the normal DNA methylation process. As all living organisms are daily exposed to numerous genome threats from both endogenous (e.g. free radicals formed during metabolism of foods) and exogenous (e.g. radiation and chemical mutagens) sources (James et al., 2003), endogenous and induced DNA damage can affect the methylation ability of DNA methyltransferases to work properly thus causing DNA hypomethylation. Particularly DNA alterations such as the accumulation of 8-oxoguanine and 5-hydroxymethylcytosine in DNA (caused by oxidative damage) are able to 
prevent binding of MeCP2 protein and thus reduce the capability of DNMTs to methylate DNA (Weitzman et al., 1994; Valinluck et al., 2004).

Similarly, the UV-light/radiation-induced pyrimidine dimers at methylated CpG sites decrease DNA methylation (Becker et al., 1985). Additionally, age is an important factor of DNA hypomethylation. Correlation between age and DNA hypomethylation has been found, as cells/organisms become less able to repair DNA damage when they age (Gorbunova et al., 2007).

It is well documented that loss of methylation in rodents results in severe developmental defects and early embryonic lethality. A number of inherited diseases in humans have also been linked to defective methylation pathways such as Rett, ICF, and ATRX syndromes. Numerous studies aimed to link the role of DNA methylation in cancer risk and progression. Aberrant methylation patterns are implicated in tumorigenesis causing genomic instability, abnormal imprinting, and deregulated expression of oncogenes or tumor suppressor genes (Zhu et al., 2006).

Figure 1.2.4 shows how hypomethylation of repeat-rich heterochromatin leads to DNA instability (a trait mark of tumor cells) and can lead to tumorigenesis, due to an increase of mitotic recombination events. Such alterations of the methylation profile are also complemented with modulation of the DNMTs expression. On the other hand, hypermethylation can arise in previously unmethylated $\mathrm{CpG}$ islands; which are usually found in the promoter regions of genes. $\mathrm{CpG}$ island hypermethylation is associated with gene silencing and arises in accordance with histone deacetylation. CpG island hypermethylation can cause silencing of tumor suppressor genes leading to cancer progression (Eden et al., 2003; Yeh et al., 2003). 


\section{Figure 1.2.4. DNA methylation and cancer.}

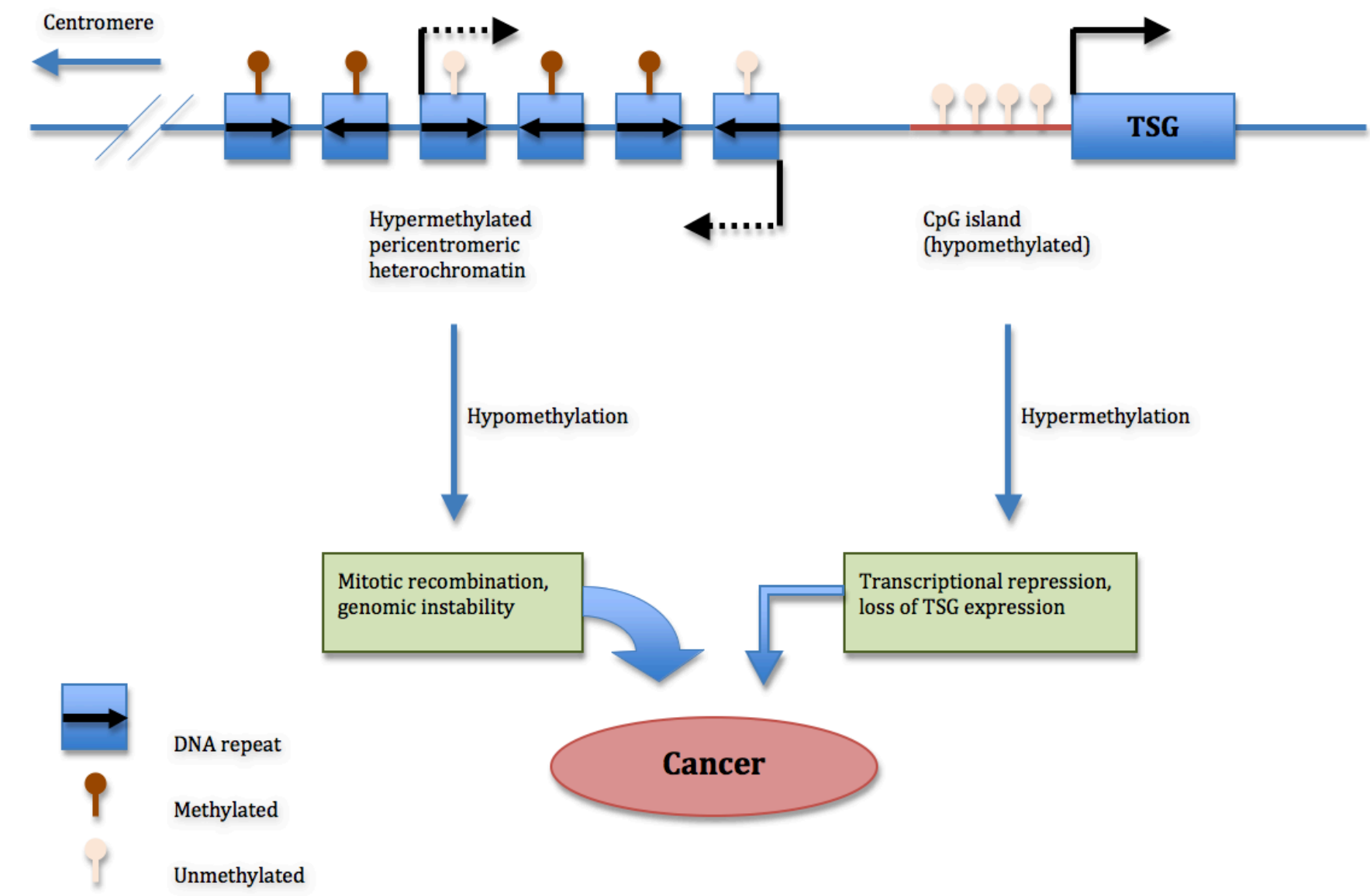

Representation of a region of genomic DNA, containing repeat-rich hypermethylated pericentromeric heterochromatin and an actively transcribed tumor suppressor gene (TSG) associated with a hypomethylated CpG island (indicated in red). Repeatrich heterochromatin becomes hypomethylated in tumor cells, contributing to genomic instability (hallmark of tumor cells) by an increase in mitotic recombination events. Moreover, de novo methylation of CpG islands can also occur in cancer cells, which could result in the transcriptional silencing of growth-regulatory genes. These changes in methylation are early events in tumorigenesis. Adapted from Phillips, 2008. 


\subsubsection{DNA hypomethylation and cancer}

Methylation studies indicate that almost all major human cancers including colon, gastric, lung, liver, breast, bladder, ovarian and endometrial, are closely related with an intense cancer-linked genome hypomethylation (Widschwendter et al., 2004; Nakagawa et al., 2005; Watts et al., 2008). Notably, the link between the extent of DNA hypomethylation and the stage and grade of cancer can be used as a biomarker, in order to diagnose and even prognose disease (Pogribny \& Beland, 2009). Nonetheless, the role of DNA hypomethylation in tumorigenesis is still unclear, and it could just be a result of malignant cell transformation revealing the undifferentiated state of tumors (Christman, 2003).

It is therefore essential to prove the significance of hypomethylation in the development of cancer, by establishing whether (a) the loss of DNA methylation occurs at a significant frequency at the initial stages of carcinogenesis, (b) whether occurring methylation changes at the preneoplastic stages are carried on till the later stages of cancer, (c) whether further changes in the methylation pattern are gained as the tumor develops and last but not least (d) whether there is an actual mechanistic link between DNA hypomethylation and the development of cancer (Pogribny \& Beland, 2009).

A number of studies have now revealed that (a) DNA methylation is usually lost during the initial stages of tumor formation, more specifically in the preneoplastic stages (Rauch et al., 2008), (b) that the greater the tumor development, the higher the degree of DNA hypomethylation (Jackson et al., 2004) and (c) that during cancer progression from the normal stage to the disease stage, multiple modifications in methylation processes are 
accumulating into the DNA (Watts et al., 2008). The results of these studies imply that the loss of DNA methylation cannot be regarded as a consequential event. It has been also found that reduction of DNA methylation by gene targeting of DNA methyl-transferase 1 (DNMT1) (Yamada et al., 2005) and lymphoid-specific helicase (Lsh) (Fan et al., 2008) can cause tumor induction, confirming the contributively role of DNA hypomethylation in the initiation of cancer.

The mechanistic link between the loss of DNA methylation and cancer development was linked to the DNA methylation landscape of the mammalian genome and the function of DNA methylation in normal cells. This was also observed in cases of induction of chromosomal instability, reactivation and transposition of retrotransposable elements, loss of imprinting, and activation of normally silenced genes. Since methylation in the mammalian genome occurs mainly at repetitive elements within the body of genes, (Rollins et al., 2006; Suzuki \& Bird, 2008) it could be hypothesized that hypomethylation of DNA mainly affects those areas.

Indeed, a strong association between global DNA hypomethylation and demethylation of repetitive sequences such as long interspersed nucleotide elements (LINE), short interspersed nucleotide elements (SINE), intracisternal A-particle elements (IAP) and Alu elements (most abundant mobile elements in the human genome) was found in tumors (Rollins et al., 2006; Suzuki \& Bird, 2008; Ogino et al., 2008). Howard and co-authors used a hypomorphic allele of DNMT1 to produce hypomethylated mouse lymphomas and reported that seven out of sixteen lymphomas recorded, had an IAP element inserted into the Notch1 gene. This led to the activation of Notch1 as an oncogene, thus initiating cancer formation. This study 
demonstrates how genomic instability, caused by hypomethylation can drive cancer progression. On the other hand, insertion of transposable elements into tumor suppressor genes can inhibit their function via insertion inactivation and that can also drive cancer cell formation (Howard et al., 2008).

As the fundamental mechanisms linking the histone and 5-methylcytosine codes are currently coming to light, the complete impact of the induction of methylation and the reinforcement of silencing of tumor-linked genes have become the centre of attention in numerous studies on the mechanisms underlying cancer initiation and progression (Jones, 2002). 


\subsection{Cancer Risk}

Classically, cancer is viewed as a group of diseases originated and compelled by critical cancer gene aberrations, (caused both by endogenous and environmental factors) that become permanent into the DNA, and consequently become inherited into the genome of new cells (Feinberg et al., 2006). It is now well documented that cancer is a disease of mutation accumulation caused by both genetic and epigenetic events (Pogribny et al., 2009a).

Nevertheless, the relative contribution of these events on carcinogenesis and cancer progression, especially when they occur in the early stages of preneoplasia, still remains elusive (Pogribny et al., 2009a). The general approach for elucidating key events for cancer development has been centred on the identification of genetic lesions linked with carcinogenesis, however it has been documented that the incidence of genetic abnormalities by itself is not enough for tumor formation (Su, 2012).

Predominantly, it is the failure of cells to preserve and control expression of genetic information accurately, which is responsible for tumorigenesis. In addition, the variety of phenotypic changes reported in preneoplastic and malignant cells cannot be explained merely by genetic alterations. These findings lead to the hypothesis that the conversion from promotion (a stage that may be reversible in carcinogenesis) to progression, (a state that may be irreversible), may be driven predominantly by epigenetic abnormalities (Su, 2012).

Piling scientific evidence points to the significance of diet as one of the most important factors in cancer development. It is known that depletion of any one of the major dietary sources of methyl groups (methionine, choline, 
folic acid and vitamin $\mathrm{B}_{12}$ ) is enough to induce tumor formation in rat and mice models. As stated by Pogribny and co-authors, the methyl-deficient model of endogenous carcinogenesis is unique in the fact that dietary omission rather than the addition of chemical carcinogens leads to the formation of tumors (Pogribny et al., 2006). Predisposition to cancer by dietary omission is caused by biochemical and molecular events leading to chronic metabolic stress thus in vivo models could be ideal in studying progressive alterations that occur at the duration of carcinogenic progress (Pogribny et al., 2006).

\subsubsection{Folate link to carcinogenesis}

Mechanisms of folate-related carcinogenesis are not yet entirely understood. It is well documented that folate is involved in numerous essential single-carbon transfer reactions, driving nucleotide syntheses (Section 1.1.4) and that it has a key role in DNA methylation (Section 1.2.1). It is also well documented that increased cancer risk in humans can be caused from disturbances in nucleotide synthesis and repair, and also from differential methylation such as hypermethylation of tumor suppressors. Moreover, global DNA hypomethylation has been linked to the progression of adenomas to cancer and the genesis of tumors, whereas gene-specific hypermethylation has been observed in critical genes in neoplastic tissues (Caudill et al., 2001; Sibani et al., 2002; Zhang et al., 2003; Hazra et al., 2007).

Folic acid deficiency could therefore lead to (1) increased amount of DNA damage which is a key cancer risk factor (2) distortion in the methylation of DNA which is a key risk factor for tumorigenesis, (3) elevation 
in the level of homocysteine in the cell which is a key risk factor for cardiovascular disease (4) developmental and neurological abnormalities (5) anaemia and (6) hyperhomocysteinemia (Fenech, 2001). Inadequate amounts of dietary intake of folate have been linked to the development of many epithelial cell cancers such as cervix, lung and breast cancer, but the most substantial indications relating folate deprivation with a higher possibility for acquiring disease, relates to colorectal cancer (Duthie et al., 2004).

Lower intake of folate has been linked to higher risk of colon cancer, while long-term use of folic acid supplements was found to decrease colon cancer risk to $75 \%$. Moreover, numerous studies have shown that a decrease in folate intake increases the risk for breast cancer, pancreatic cancer in smokers, gastric and oesophageal cancer, acute lympoblastic leukaemia $(A L L)$ in children and acute lymphocytic leukaemia (ALL) in adults. Folate deprivation has been further linked to an increase risk for developing cervical squamous epithelial lesions leading to cervical cancer as show in a case control study (Ames \& Wakimoto, 2002).

In humans, evidence revealing the link between cancer and folate intake was obtained by studying the effects of a polymorphism (C677T) in the gene C677 encoding methyleneTHF reductase (MTHFR). This polymorphism reduces the activity of MTHFR, which leads to the increase of the methyleneTHF amounts in a cell and the decrease of the methyl-THF, leading to an increased integration of uracil in the genome and a rise in chromosomal breakage (Figure 1.3.1). This polymorphism is common in human populations living in northern regions of the world, with $5-25 \%$ of the individuals being homozygous to the polymorphism while $50 \%$ being heterozygous (Ames \& Wakimoto, 2002). 
Figure 1.3.1 Link of folate deficiency and cancer.
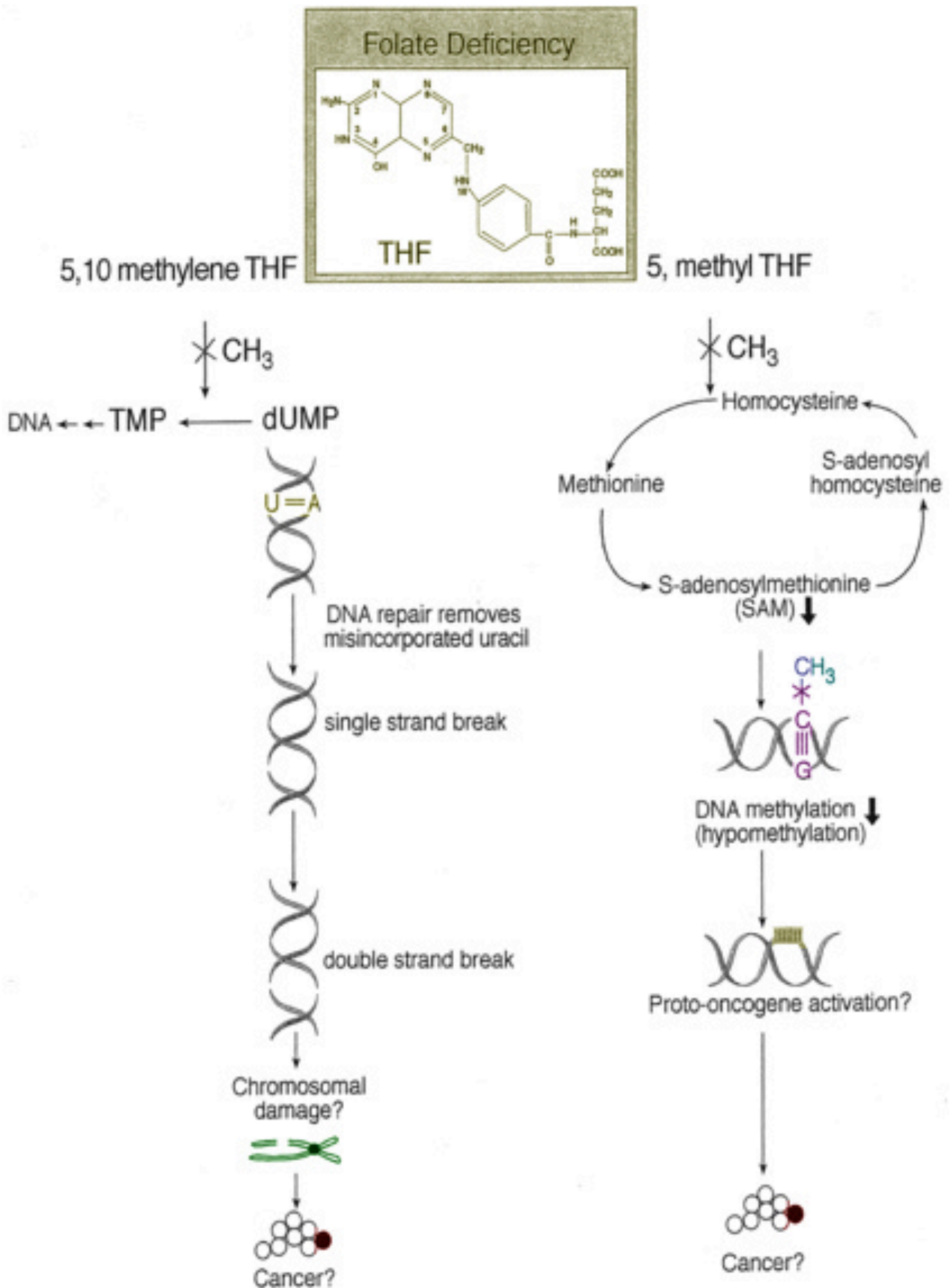

Adapted from Duthie et al., 2002. 
Numerous studies have recorded a two-fold decrease in the risk of colon cancer in people that are homozygous to the polymorphic C677T allele of MTHFR when compared to the homozygous individuals with the normal C677 that have a higher folate intake. However, at low levels of folate the C677T genotype was found to be a risk factor for disease. Studies have recorded that there is an increase in the risk for developing adenomatous polyps, adult acute lymphocytic leukaemia (ALL) and childhood acute lymphoblastic leukaemia (ALL) with the C677T genotype, showing that folate deficiency promotes ALL. Lower levels of MTHFR that are also caused by the C677T genotype, decrease the methylTHF pool, which in its turn increases the levels of homocystein; a risk factor for endothelial-cell damage and cardiovascular disease (Ames \& Wakimoto, 2002).

The present knowledge on folate deficiency and its role to cancer, highlights the importance of multidisciplinary studies, including molecular, epidemiological and clinical, in furthering the understanding of the links between diet and cancer (Ames \& Wakimoto, 2002). As the mechanism underlining cancer risk and folate-deficiency still remains indefinable, two important questions must be raised in order to understand the link between folate and cancer (a) can methyl-deficient diet affect genome stability and (b) are the effects of a methyl-deficient diet permanent? 


\subsubsection{Epidemiological evidence}

The first study on humans for folate and vitamin $B_{12}$ deficiency revealed the expression of Howell-Jolly bodies (chromosomes or chromosome fragments that lag behind at the anaphase stage of the production and maturation of red blood cells, similar to micronuclei) in erythrocytes of patients with megaloblastic anaemia due to the occurrence of chromosomal damage (Discombe, 1948; Koyama, 1960; Lessin \& Bessis, 1972). Usually, in normal individuals, any formed micronuclei are removed by spleen (Smith et al., 1990).

A case study on a patient suffering from Chron's disease found an unusually high level of micronuclei in the erythrocytes, which was attributed to a low amount of folate. After treatment with a daily oral dose of $15-20$ $\mathrm{ng} / \mathrm{mg}$ (higher than the normal doses) for 25 days, the frequency of micronuclei in this patient was stabilized (Everson et al., 1988). Another study on smokers and non-smokers showed a significant relationship between smoking and low levels of folate in the blood, which causes chromosomal fragility and leads to aberrations (Chen et al., 1989).

A study on the effects of blood micronutrients on micronucleus frequency in erythrocytes, revealed that increases in the micronucleus index were strongly associated with low levels of serum folate. On the contrary, vitamins C, E and beta-carotene showed no association with the levels of micronucleus index (MacGregor et al., 1997). Blood from the same individuals was analysed for uracil content (Everson et al., 1988; Chen et al., 1989) and was found that the rate of uracil misincorporation had a 70 -fold increase in people whose serum folate was lower than $4 \mathrm{ng} / \mathrm{ml}$ compared with people whose serum folate was above $4 \mathrm{ng} / \mathrm{ml}$ (Blount et al., 1997). 
It was further discovered that levels of uracil misincorporation in the DNA were reduced within three days of daily folic acid supplementation in a dose of $5 \mathrm{mg}$ in both deficient and non-deficient groups. These changes were found to coincide with reduced erythrocyte micronucleus frequencies (Blount et al., 1997).

A study based on both folate depleted and sufficient diets on a small group of post-menopausal women, recorded a significant increase in micronucleus frequency in lymphocytes after depletion of folate and a decrease of micronucleus frequency after folate supplementation, especially in buccal cells. At the folate depletion phase, increases in DNA hypomethylation and in the dUTP/dTTP ratio, as well as lower NAD levels in lymphocytes were also recorded (Fenech, 2001).

Another study on the effects of folate levels revealed that buccal mucosal folate and vitamin $\mathrm{B}_{12}$ are considerably lower in smokers than in non-smokers (Piyathilke et al., 1995). Data revealed that smokers had a 3fold increase in micro nucleated buccal cells when compared to the nonsmokers group. The study aimed to investigate whether epithelial cancers such as those of the cervix, lung, bladder and/or pharyngeal region could be the result of localised deficiencies in folic acid and vitamin $B_{12}$, and the findings revealed that folate and vitamin $\mathrm{B}_{12}$ are able to correct megaloblastic changes in these tissues (Krumdieck, 1991).

Table 1.3.1 shows a summary of a number of publications investigating the relationship between dietary folate deprivation and a variety of cancers. 
Table 1.3.1. Summary of the results of studies investigating the relationship between dietary folate status and cancer susceptibility.

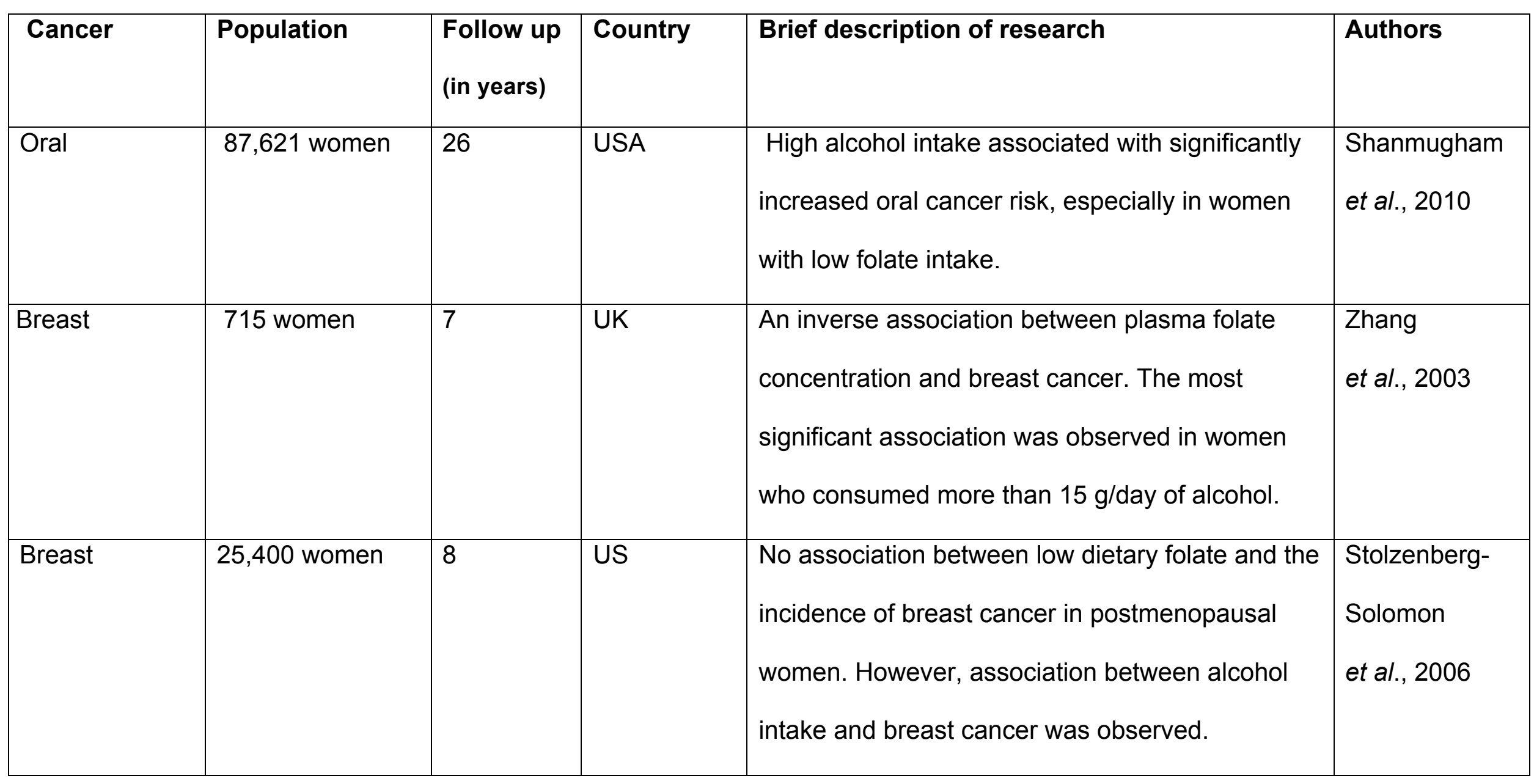




\begin{tabular}{|c|c|c|c|c|c|}
\hline Breast & 11,699 women & 10 & Sweden & $\begin{array}{l}\text { High folate intake linked with lower breast cancer } \\
\text { incidence in postmenopausal women. }\end{array}$ & $\begin{array}{l}\text { Ericson } \\
\text { et al., } 2007\end{array}$ \\
\hline Breast & 3,116 women & 21 & Sweden & $\begin{array}{l}\text { Folate intake before breast cancer diagnosis may } \\
\text { improve breast cancer and overall survival }\end{array}$ & $\begin{array}{l}\text { Harris } \\
\text { et al., } 2012\end{array}$ \\
\hline Cervical & $\begin{array}{l}39 \text { participants } \\
\text { with cervical } \\
\text { cancer, } 2 \text { controls } \\
\text { per case }\end{array}$ & l & $\begin{array}{l}\text { Washington, } \\
\text { USA }\end{array}$ & $\begin{array}{l}\text { No statistically significant association between } \\
\text { folate, B12 and cervical cancer was found. }\end{array}$ & $\begin{array}{l}\text { Alberg } \\
\text { et al., } 2000\end{array}$ \\
\hline Cervical & 329 women & 5 & Hawaii & $\begin{array}{l}\text { Dietary intakes of folate, B6 and B12 were } \\
\text { inversely related to the risk of developing cervical } \\
\text { dysplasia }\end{array}$ & $\begin{array}{l}\text { Goodman } \\
\text { et al., } 2001\end{array}$ \\
\hline Colorectal & 1021 participants & 10 & USA \& & Folic acid supplementation of $1 \mathrm{mg}$ a day not & Cole \\
\hline
\end{tabular}




\begin{tabular}{|c|c|c|c|c|c|}
\hline & $\begin{array}{l}\text { with history of } \\
\text { colorectal } \\
\text { adenomas. }\end{array}$ & & Canada & $\begin{array}{l}\text { found to be protective. The frequency of } \\
\text { reoccurrence was higher in participants taking } \\
\text { folic acid than those on placebo, suggesting } \\
\text { elevated folate as a risk factor for CRC. }\end{array}$ & et al., 2007 \\
\hline Colorectal & 47,000 men & l & US & $\begin{array}{l}\text { Weak association between low dietary folate and } \\
\text { incidence of colorectal cancer. The association } \\
\text { was much stronger when folate intake was in } \\
\text { combination with high alcohol intake. }\end{array}$ & $\begin{array}{l}\text { Giovannucci } \\
\text { et al., } 1995\end{array}$ \\
\hline Colorectal & $\begin{array}{l}\text { 47,931 men } \\
\text { (ages 40s) }\end{array}$ & 1 & US & $\begin{array}{l}\text { Folate intake of } 400 \mu \mathrm{g} \text { a day or higher was } \\
\text { shown to reduce the incidence of colorectal } \\
\text { cancer, in women, when compared to women } \\
\text { with a folate intake of } 200 \mu \mathrm{g} \text { a day or lower. }\end{array}$ & $\begin{array}{l}\text { Giovannucci } \\
\text { et al., } 1998\end{array}$ \\
\hline $\begin{array}{l}\text { Colorectal } \\
\text { cancer, } \\
\text { adenomas }\end{array}$ & $\begin{array}{l}20 \text { participants } \\
\text { with adenomas }\end{array}$ & 1 & $\begin{array}{l}\text { Toronto, } \\
\text { Canada }\end{array}$ & $\begin{array}{l}\text { Lower intake of folate was associated with higher } \\
\text { risk of colon cancer; } \\
\text { Long-term use of folate supplement lowers the } \\
\text { risk of colon cancer by } 75 \% \text {. }\end{array}$ & $\begin{array}{l}\text { Kim et al., } \\
2001 \text {; } \\
\text { Giovannucci } \\
\text { et al., } 1998\end{array}$ \\
\hline
\end{tabular}




\begin{tabular}{|c|c|c|c|c|c|}
\hline Colorectal & $\begin{array}{l}200 \text { women with } \\
\text { colorectal } \\
\text { hyperplastic } \\
\text { polyps and } 654 \\
\text { controls }\end{array}$ & 5 & $\begin{array}{l}\text { Minnesota, } \\
\text { U.S.A }\end{array}$ & $\begin{array}{l}\text { No association was seen between folate, } B_{6} \text { and } \\
B_{12} \text { and risk of colorectal } 146 \text { hyperplastic polyps. }\end{array}$ & $\begin{array}{l}\text { Ulrich } \\
\text { et al., } 2000\end{array}$ \\
\hline Colorectal & 781 participants. & 4 & $\begin{array}{l}\text { North } \\
\text { America }\end{array}$ & $\begin{array}{l}\text { The effect of folic acid on risk of adenomas did } \\
\text { not differ according to the extent of LINE-1 } \\
\text { methylation and no association between LINE-1 } \\
\text { methylation and risk of adenomas was found. }\end{array}$ & $\begin{array}{l}\text { Figueiredo } \\
\text { et al., } 2009\end{array}$ \\
\hline Colorectal & $\begin{array}{l}602 \mathrm{ppl} \text { with the } \\
\text { MTHFR polym. } \\
\text { and } 602 \text { controls. }\end{array}$ & l & $\begin{array}{l}\text { Massachus } \\
\text { etts, } \\
\text { US }\end{array}$ & $\begin{array}{l}\text { Low plasma folate levels are associated with } \\
\text { lower risk of colorectal cancer. }\end{array}$ & $\begin{array}{l}\text { Lee } \\
\text { et al., } 2012\end{array}$ \\
\hline
\end{tabular}




\begin{tabular}{|c|c|c|c|c|c|}
\hline Colorectal & $\begin{array}{l}787 \text { participants } \\
\text { with MTHFR } \\
\text { polymorphisms, } \\
\text { and } 656 \text { control } \\
\text { participants. }\end{array}$ & I & Korea & $\begin{array}{l}\text { High folate intake associated with reduced } \\
\text { colorectal cancer risk. However the effect of } \\
\text { dietary methyl supply on colorectal } \\
\text { carcinogenesis may differ according to MTHFT } \\
\text { C677T genotype and the subside of origin in a } \\
\text { population. }\end{array}$ & $\begin{array}{l}\text { Kim } \\
\text { et al., } 2012\end{array}$ \\
\hline Lung & $\begin{array}{l}230 \text { men and } 102 \\
\text { women with lung } \\
\text { cancer, } 597 \text { men } \\
\text { and } 268 \text { women } \\
\text { controls. }\end{array}$ & 2 & Hawaii & $\begin{array}{l}\text { No association between folic acid intake and the } \\
\text { incidence of lung cancer. }\end{array}$ & $\begin{array}{l}\text { Le Marchand } \\
\text { et al., } 1989\end{array}$ \\
\hline Lung & $\begin{array}{l}27,544 \text { men } \\
(395 \text { cases }) \text { and } \\
20,456 \text { women } \\
\text { (130 cases). }\end{array}$ & 7 & $\begin{array}{l}\text { New York, } \\
\text { US }\end{array}$ & $\begin{array}{l}\text { Inverse relationship between folate intake and } \\
\text { lung cancer, in male participants but not female. }\end{array}$ & $\begin{array}{l}\text { Bandera } \\
\text { et al., } 1997\end{array}$ \\
\hline
\end{tabular}




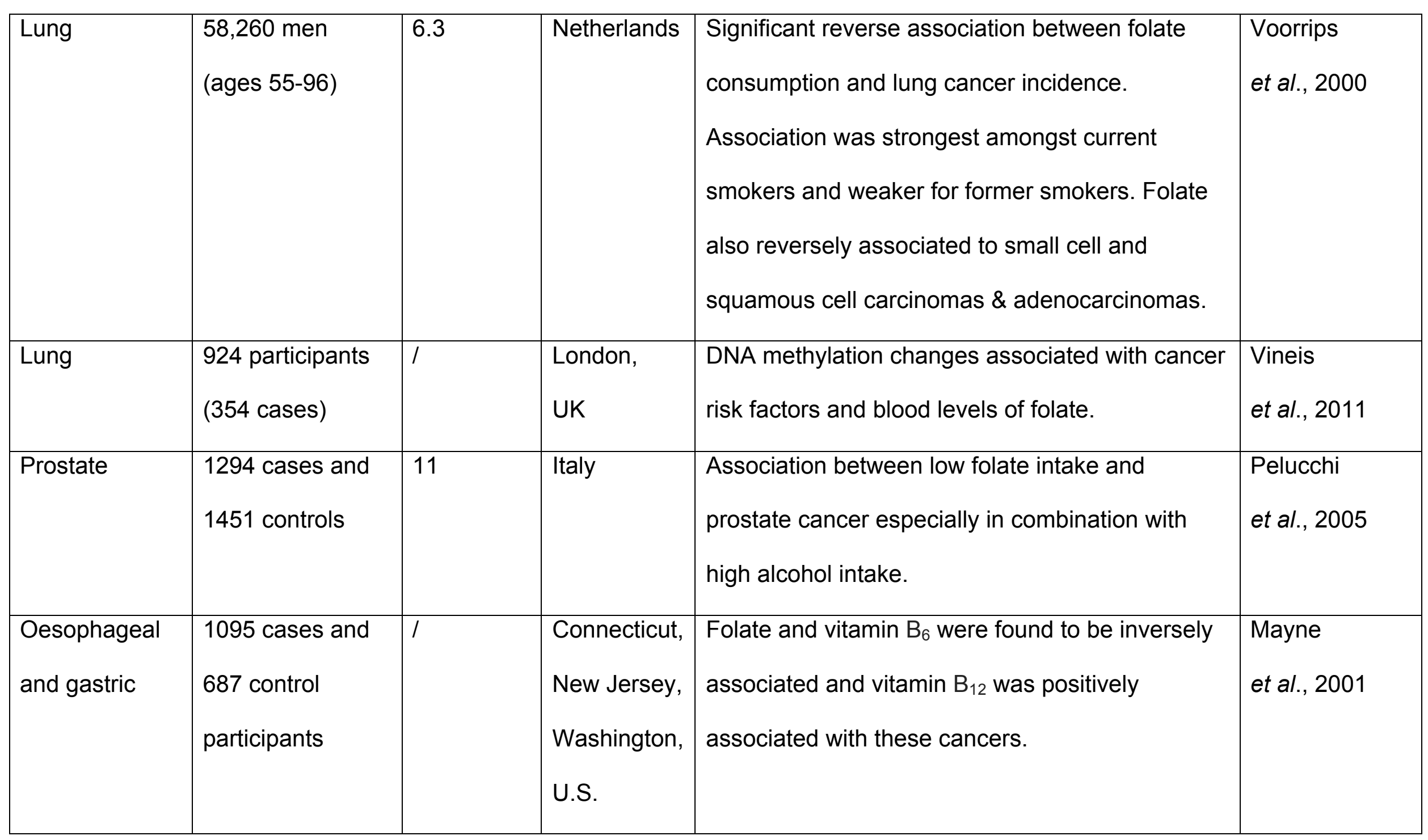




\begin{tabular}{|c|c|c|c|c|c|}
\hline $\begin{array}{l}\text { Pancreatic } \\
\text { cancer }\end{array}$ & $\begin{array}{l}126 \text { cases and } \\
247 \text { control } \\
\text { participants. } \\
\text { All smokers } \\
\text { (ages } 50-69 \text { ) }\end{array}$ & 5 & Finland & $\begin{array}{l}\text { Risk of pancreatic cancer in smokers was } \\
\text { inversely associated with dietary folate. }\end{array}$ & $\begin{array}{l}\text { Stolzenberg- } \\
\text { Solomon et al., } \\
\text { 1999; } 2001\end{array}$ \\
\hline $\begin{array}{l}\text { Pancreatic } \\
\text { cancer }\end{array}$ & 77,640 women & 14 & US & $\begin{array}{l}\text { No statistically strong association between } \\
\text { energy-adjusted folate intake and the risk of } \\
\text { pancreatic cancer. }\end{array}$ & $\begin{array}{l}\text { Skinner } \\
\text { et al., } 2004\end{array}$ \\
\hline $\begin{array}{l}\text { Pancreatic } \\
\text { cancer }\end{array}$ & $\begin{array}{l}120,852 \\
\text { participants } \\
\text { (ages } 55-69 \text { ) }\end{array}$ & 13.3 & Netherlands & $\begin{array}{l}\text { No support of a protective association of total } \\
\text { dietary folate or individual folate vitamers on the } \\
\text { risk of pancreatic cancer. }\end{array}$ & $\begin{array}{l}\text { Keszei } \\
\text { et al., } 2009\end{array}$ \\
\hline $\begin{array}{l}\text { Liver: } \\
\text { (HCC) }\end{array}$ & $\begin{array}{l}120 \text { patients and } \\
120 \text { controls }\end{array}$ & 4 & $\begin{array}{l}\text { Taiwan, } \\
\text { China }\end{array}$ & $\begin{array}{l}\text { The mt genetic instability that results from a high } \\
\text { rate of mtDNA deletions and/or low folate status } \\
\text { increased the risk for HCC. }\end{array}$ & Wu et al., 2009 \\
\hline
\end{tabular}




\begin{tabular}{|c|c|c|c|c|c|}
\hline $\begin{array}{l}\text { Lymphoblastic } \\
\text { leukaemia } \\
\text { (ALL; children) }\end{array}$ & $\begin{array}{l}253 \text { All and } 200 \\
\text { controls }\end{array}$ & 1 & UK & $\begin{array}{l}\text { ALL was associated with the MTHFR genotypes, } \\
\text { indicating a role of folate in the development of } \\
\text { ALL. }\end{array}$ & $\begin{array}{l}\text { Wiemels } \\
\text { et al., } 2001\end{array}$ \\
\hline $\begin{array}{l}\text { Acute } \\
\text { lymphoblastic } \\
\text { leukaemia } \\
\text { (ALL; adults) }\end{array}$ & $\begin{array}{l}308 \text { patients and } \\
491 \text { age and sex } \\
\text { matched controls }\end{array}$ & 1 & UK & $\begin{array}{l}\text { A significant reduction in risk of ALL was found in } \\
\text { those with the MTHFR } 677 T T \text { genotype, } \\
\text { indicating that folate deficiency might be a risk } \\
\text { factor. }\end{array}$ & $\begin{array}{l}\text { Skibola } \\
\text { et al., } 1999\end{array}$ \\
\hline $\begin{array}{l}\text { Head and neck } \\
\text { squamous cell } \\
\text { carcinoma } \\
\text { (HNSCC) }\end{array}$ & 242 patients & 1 & $\begin{array}{l}\text { Boston, } \\
\text { US }\end{array}$ & $\begin{array}{l}\text { Dietary folate associated with p16INK4A } \\
\text { methylation in head and neck squamous cell } \\
\text { carcinoma. }\end{array}$ & $\begin{array}{l}\text { Kraunz et al. } \\
2006\end{array}$ \\
\hline
\end{tabular}

Adapted from Ames \& Wakimoto, 2002. 
As shown in Table 1.3.1, experimental evidence linking folate deficiency to cancer is not consistent. There are numerous papers linking folate to most common cancer types, however, in the majority of cases there are conflicting studies, failing to record significant association between folate intake and incidence of cancer (Kim 1999a; Kim, 1999b; Eichholzer et al, 2001). Due to the mixed findings from previous publications, more research is needed to conclusively determine if folate deficient diets can increase cancer susceptibility. However, as many of the larger studies support the association, there is reason to believe that folate deficiency does affect cancer susceptibility (Fenech, 2001). In vitro and in vivo animal studies might be able to shed some light in to the elusive mechanisms linking folate deficiency and cancer incidence, where population studies have not yet been successful.

\subsubsection{In vitro studies}

Numerous in vitro studies with human lymphocytes have revealed that folic acid deprivation causes expression of chromosomal fragile sites, as well as increased chromosomal breakage and micronucleus expression (MN), which suggests a common mechanism underlying the expression of fragile sites and chromosome breaks. Folic acid deprivation in lymphocyte cells cultured in vitro showed an increase in the level of excision repair during the G2 cell stage due to the two-fold increase in uracil misincorporation recorded into the DNA (Fenech, 2001).

According to the results of an in vitro study, folate supplementation in folic-acid deficient lymphocyte DNA48 cells caused reduction of uracil incorporation into the DNA and reduction of chromosomal breaks. It was also 
reported that all markers of chromosomal damage minimized when folic acid concentrations were higher than the RDA. Even though the specific study did not examine cancer incidence, chromosomal breaks have been linked to cancer in a plethora of other studies (Ames \& Wakimoto, 2002).

When human lymphoid cells where treated in vitro with methotrexate (an anticancer drug that inhibits the metabolism of folic acid) a significant increase in the dUTP/dTTP ratio was recorded and a higher increase of uracil incorporation into the DNA (Goulian et al., 1980), resulting in an increased DNA strand breakage (Duthie \& Hawdon, 1998). All these studies link folate and genome stability (Fenech, 2001).

Bistulfi and co-authors studied the consequences of long-term, mild folate depletion in a model comprised of three syngenic cell lines derived from the transgenic adenoma of the mouse prostate (TRAMP) model, recapitulating different stages of prostate cancer; beginning, transformed and metastatic. The data revealed that mild folate depletion (100 nM) was sufficient to cause imbalance in the nucleotide and AdoMet pools in all prostate cell lines, significant increase in uracil misincorporation and DNA single strand breaks. Five novel chromosomal rearrangements were also recorded in cells grown with mild folate depletion (Bistulfi et al., 2010).

An increase in $\mathrm{CpG}$ island and histone methylation upon folate depletion was also identified, despite unchanged levels of total 5methylcytosine, demonstrating a wide-ranging effect of folate depletion on epigenetic regulation. The genomic changes reported, coincided with phenotypic alterations in the prostate cells that include reduced sensitivity to folate depletion. In conclusion, this study demonstrated the high susceptibility of prostate cells to genetic and epigenetic changes subsequent to mild folate 
depletion as compared with cells grown to folate levels $(2 \mu \mathrm{M})$ routinely used in tissue cultures (Bistulfi et al., 2010).

Carroll and co-authors examined the molecular response to folate metabolism inhibition by exposing human lymphoblast cell lines to the methionine adenosyltransferase inhibitor cycloleucine. The authors identified 4 genes that were considered reliable responders to cycloleucine treatment (CXCR3, PGDS, GPR98 and CBS) and the gene products were all found related either to immune cells, or the immune response. Furthermore, genespecific DNA methylation analysis revealed no evidence that DNA methylation changes were mediating the gene expression changes observed. When the data from this study were compared with other gene expression studies on folate deficiency, it was revealed that all studies found a link between gene products related to immune cells or immune response and cellular response to folate status (Carroll et al., 2012).

One of the latest in vitro studies, aimed to examine the role of folate deficiency on the formation of complex nuclear abnormalities in cytokinesisblocked binucleated cells (Bull et al., 2012). It is well established that chromosomal instability is an essential hallmark of oncogenesis and that it can be diagnosed morphologically by the presence of nuclear anomalies such as micronuclei, nucleoplasmic bridges, and nuclear buds. The authors were able to identify additional nuclear anomalies formed under folatedeficient conditions, defined as fused nuclei (FUS), circular nuclei (CIR), and horseshoe nuclei (HS). A high proportion of fusion regions that contained both centromeric and telomeric DNA was also recorded, suggesting that folate deficiency can possibly disrupt the process of sister chromatid separation and chromosome segregation during mitosis (Bull et al., 2012). 


\subsubsection{In vivo animal studies}

In vivo studies in rodents (either with depletion of folate in diet, or use of antibiotics that eliminated folate producing bacteria in the gut) have confirmed the results found in vitro, of elevation in DNA strand breaks, DNA hypomethylation and increase in uracil misincorporation in the DNA (Table 1.1.3). However, marginal folic acid depletion in the daily diet (400 nmol/kg diet) was not found to increase the micronucleus levels either in maternal or in fetal erythrocytes (Fu et al., 1996). Sibani and his group created a dimethyl-hydrazine rat model of colon carcinogenesis in which folate deficiency was found to increase tumor prevalence. On the other hand folate supplemented rats were found to have protection against tumor formation, suggesting a cause-and-effect relationship between folate and tumor incidence (Sibani et al., 2002). These data have therefore provided a vital evidence for the effects of folate intake levels on the genomic stability in vivo.

One of the most thoroughly studied models of liver cancer is the methyl-deficient liver carcinogenesis in rats. This model is unique as it clearly demonstrates that dietary omission of methyl group-sources is on its own capable of causing tumor formation. The sequence of pathological and molecular events in this model is extremely similar to the initiation of human hepatocellular carcinomas by viral hepatitis B and C infections, alcohol exposure, and metabolic liver diseases (Pogribny et al., 2009a).

It is thought that the effects of a methyl-deficient diet on liver carcinogenesis may be attributed to early oxidative DNA damage, however the mechanisms underlying these alterations in liver carcinogenesis and whether these alterations can be considered as neoplastic still remain unclear. Pogribny and co-authors investigated the contribution of DNA 
damage and epigenetic alterations to liver carcinogenesis induced by a methyl-deficient diet in rats. The data from this study revealed that a 9-week duration on a methyl-deficient diet had a profound effect on liver DNA, via an early up-regulation of base excision repair genes, an accumulation of 8oxodeoxyguanosines and $3^{\prime} \mathrm{OH}$-end strand breaks, leading to global loss of DNA methylation, hypermethylation of $\mathrm{CpG}$ islands and DNA damage. The authors stated that these abnormalities were fully restored in the livers of rats, that were switched to a methyl-sufficient diet after the end of the 9-week treatment (Pogribny et al., 2009a).

It is important to note that the liver tissue of rats that were fed a methyl-deficient diet for a bigger duration of time (18 weeks) before returned to a methyl-sufficient diet was unable to restore all abnormalities, and only DNA lesions were repaired. The methyl-sufficient diet was therefore unable to restore the pre-diet level of DNA methylation status and was not able to prevent the progression of liver carcinogenesis. These findings show that stable alterations in DNA methylation patterns can facilitate progression of liver carcinogenesis and suggest that epigenetic changes may be better markers than DNA lesions of the carcinogenic process and carcinogen exposure (Pogribny et al., 2009a).

Another important mouse model employed for the investigation of intestinal cancer is the $\mathrm{Apc}^{\mathrm{Min} /+}$ mouse. This mouse model has a genetic mutation in the tumor suppressor gene, adenomatous polyposis coli $(A p c)$ and is homologous to the human $A p c$ germ-line mutations that cause the hereditary condition familial adenomatous polyposis (FAP) (Luongo et al., 1994). Individuals with FAP have elevated risk of developing adenomatous colon polyps (Groden et al., 1991). The recorded number of adenoma 
formation can range from hundreds to thousands, and if left untreated are expected to develop into colorectal cancer (Sieber et al., 2003).

FAP and Gardner's syndrome (FAP with osteomas of the skull, epidermoid cysts, dental anomalies, and/or desmoid tumors) result from mutations in the Apc gene (Jasperson et al., 2010). The Apc mutations have also been shown to promote the initiation of the majority of sporadic colorectal cancers (Banerjee et al., 1998; Polakis, 2000; Fodde et al., 2002). Moreover, inactivation of the Apc gene was recorded in $80 \%$ of sporadic colorectal cancer cases (Kinzler et al., 1996).

Even though folate has been implicated in colorectal cancer risk in both epidemiological and animal studies, the results of these studies still remain inconclusive, with some studies showing restriction to have tumorinhibitory effects, whereas others suggest excess of adverse outcomes.

Table 1.3.2 presents a summary of these studies conducted to investigate the role of dietary folate restriction or excess in intestinal tumor development in the $\mathrm{Apc}^{\mathrm{Min} /+}$ mouse model, which is genetically prone to develop colorectal cancers (Teh et al., 2012). 
Table 1.3.2. Summary of studies using the Apc ${ }^{\mathrm{Min} /+}$ mouse model to examine the effect of folate on tumor development and genome stability.

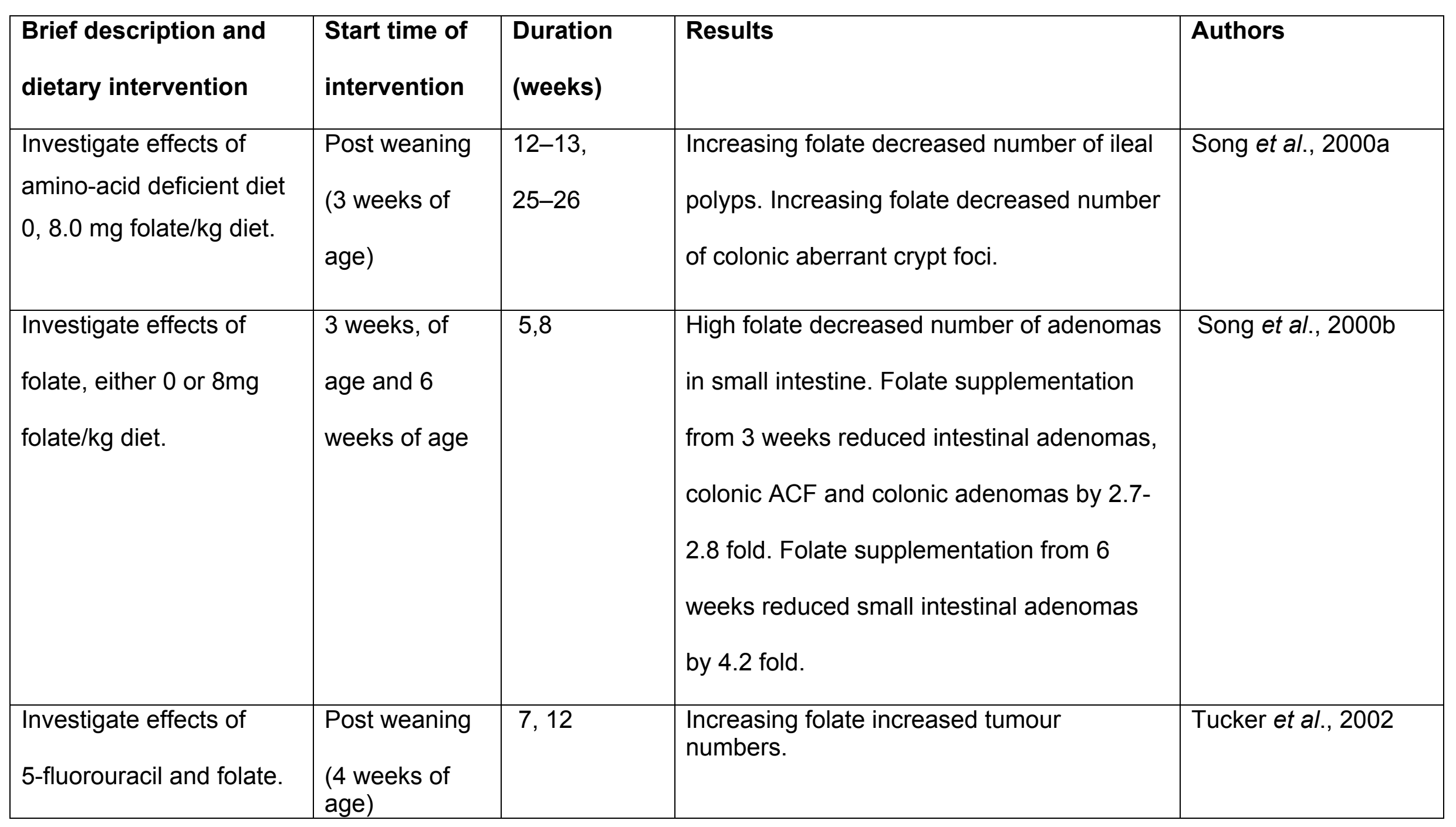




\begin{tabular}{|c|c|c|c|c|}
\hline $\begin{array}{l}\text { Investigate DNMT1 and } \\
\text { folate deficiency effects: } \\
0 \text { and } 2 \mathrm{mg} \text { folate/kg diet } \\
\text {. }\end{array}$ & $\begin{array}{l}\text { Post weaning } \\
\text { ( } 3 \text { weeks of } \\
\text { age) }\end{array}$ & 10 & $\begin{array}{l}\text { Folate/choline did not produce a consistent } \\
\text { effect on tumour number. Folate/choline } \\
\text { deficiency decreased SAM. Folate/choline } \\
\text { deficiency increased global DNA } \\
\text { hypomethylation. } \\
\text { Positive correlation between SAM, SAH or } \\
\text { DNA methylation with tumour multiplicity. }\end{array}$ & Sibani et al., 2002 \\
\hline $\begin{array}{l}\text { Folate deficiency, } \\
0,2.0 \mathrm{mg} \text { folate } / \mathrm{kg} \text { diet. }\end{array}$ & $\begin{array}{l}\text { Post weaning } \\
\text { ( } 3 \text { weeks of } \\
\text { age) }\end{array}$ & 10 & $\begin{array}{l}\text { Folate deficiency decreased tumour number } \\
\text { in } A p c^{M i n(+)} \mathrm{DNMT}^{(+/)} \text {, but not in } \\
\mathrm{Apc}^{\mathrm{Min}(+/)} \mathrm{DNMT}^{(+/+)} \text {mice. }\end{array}$ & Trasler et al., 2003 \\
\hline $\begin{array}{l}\text { Investigate folate } \\
0.3,1.0,2.0 \mathrm{mg} \text { folate. }\end{array}$ & $\begin{array}{l}\text { Post weaning } \\
\text { (3 weeks of } \\
\text { age) }\end{array}$ & 8 & $\begin{array}{l}\text { Reduced or increased folate conditions in } \\
\text { parallel with corresponding multivitamin } \\
\text { changes increased number of polyps and } \\
\text { the tumour burden, crypt fission and weight } \\
\text { of small intestine. }\end{array}$ & Bashir et al., 2004 \\
\hline
\end{tabular}




\begin{tabular}{|c|c|c|c|c|}
\hline $\begin{array}{l}\text { Investigate folate } \\
\text { deficiency effects. } 0.2- \\
0.3,2.0 \mathrm{mg} \text { folic acid/ kg. }\end{array}$ & $\begin{array}{l}\text { Post weaning } \\
\text { (3 weeks of } \\
\text { age) }\end{array}$ & 7 & $\begin{array}{l}\text { Folate deficiency increased adenoma } \\
\text { number, plasma homocysteine, and } \\
\text { apoptosis in all genetic backgrounds. }\end{array}$ & Lawrance et al., 2007 \\
\hline $\begin{array}{l}\text { Investigation of in utero } \\
\text { folate deficiency effects: } \\
0.26,0.4,2.0 \mathrm{mg} \text { folic } \\
\text { acid/ kg diet. }\end{array}$ & $\begin{array}{l}\text { In utero and } \\
\text { after birth }\end{array}$ & 10 & $\begin{array}{l}\text { Folate deficiency decreased global DNA } \\
\text { methylation in all genetic backgrounds. } \\
\text { No significant effect of folate dose on tumor } \\
\text { number or size. }\end{array}$ & McKay et al., 2008 \\
\hline $\begin{array}{l}\text { Investigate MTHFR and } \\
\text { folate effects: } \\
0.3,2.0,20.0 \mathrm{mg} \\
\text { folate/kg diet } \\
0.3,2.0,20.0 \mathrm{mg} \\
\text { folate/kg diet } \\
\text { (amino acid deficient } \\
\text { diet). }\end{array}$ & $\begin{array}{l}\text { Dietary } \\
\text { intervention } \\
\text { started prior to } \\
\text { mating, } \\
\text { throughout } \\
\text { pregnancy and } \\
\text { nursing. }\end{array}$ & $7+$ & $\begin{array}{l}\text { High folate diet from weaning developed } \\
\text { more adenomas. Offspring of mice on low } \\
\text { folate diet and subsequently on the same } \\
\text { diet post-weaning developed fewer } \\
\text { adenomas MTHFR }{ }^{+/} \text {genotype associated } \\
\text { with reduced adenoma numbers in the }\end{array}$ & Lawrance et al., 2009 \\
\hline
\end{tabular}

Adapted from Teh et al., 2012. 
As seen in the table above, although some studies report that folate deficiency tends to increase the risk of tumor formation, there are contradictions regarding whether excess of folate post-weaning or after tumor initiation increases intestinal tumor burden. Overall, the collective data do not seem to show changes in intestinal tumor frequency between post-weaning diets that are folate deficient or adequate (Teh et al., 2012).

With the limited literature available, the mechanisms by which dietary folate affects tumorigenesis in the $\mathrm{Apc}^{\mathrm{Min} /+}$ mouse model remain unclear and speculative. Even though the model has been widely employed for the investigation of dietary effects on intestinal cancer, data reveal that mice mostly develop small intestinal polyps compared to humans, who mainly develop intestinal cancer in the colon (Song et al., 2000b; Myant et al., 2011). Since these inconsistencies exist amongst studies, further research based on mechanisms that include modulation of nucleotide synthesis, DNA methylation, and chromosomal instability (which may affect the rate of cellular division and its control) is warrant (Teh et al., 2012).

Additionally, it remains to be seen whether the effects of folate deprivation may be reflected on intestinal cancer in wild type or genetically normal mice as well. A study conducted by Newmark and co-authors showed an increase in intestinal neoplasms in normal C57/BL6 mice fed a westernstyle diet (reduced calcium, vitamin D, folic acid, methionine, choline and vitamin $B_{12}$ ) to a degree resembling that consumed by western human populations (Newmark et al., 2001). Follow up studies using the same model showed that the western-style diet caused gene expression changes in colonic epithelium comparable to that induced by inheritance of a mutant $A p c$ 
gene (Yang et al., 2008; Newmark et al., 2009).

It is apparent that attention must be given to mechanisms by which folate affects gene expression; e.g. both folate deficiency/excess can modify histone methylation and micro RNA profiles, but their impact in intestinal carcinogenesis is still unclear (Tryndyak et al., 2009; Piyathilake et al., 2010). For that reason, folate dependent tumorigenesis mechanisms such as (a) changes in histone and DNA methylation status, (b) assessment of chromosomal stability and DNA damage, (c) the influence of SAM: SAH ratio, (d) interaction between $A p c$ and polyamines and (e) DNA methyltransferase expression and activity, should be further studied, in order to provide stronger evidence of the role of folate and other methyl donors on tumor promotion or protection (Teh et al., 2012).

The role of folate and other methyl donors, on tumor cell proliferation will eventually depend on the unique genetic changes occurring in cancer cells, which should clear up some of the contradictions reported between and within experiments (Teh et al., 2012).

\subsection{Transgenerational instability}

To date, several scientists have recorded indisputable evidence for mutation induction in the germline of parents exposed to wide range of biological stressors, including, for example, ionizing radiation, chemicals and early life stress (ELS). However, the results of numerous publications suggest that these stressors may also have an indirect effect on genome stability, which is passed on through the germline of the exposed parents to their offspring. This phenomenon is known as transgenerational instability and it is considered as a delayed risk factor in human populations. The way 
transgenerational instability is manifested in the progeny of exposed parents till this day remains elusive, even though it has been suggested that the ability of an external agent to stimulate transgenerational instability requires an epigenetic phenomenon most probably attributed to changes in the pattern of DNA methylation (Barber et al., 2002; Dubrova, 2003; Anway et al., 2005; Barber et al., 2006b).

\subsubsection{Epigenetic reprogramming}

There are two specific epigenetic DNA methylation-reprogramming cycles that have been extensively characterized and could be linked to the transgenerational passing of information to the offspring. The first occurs during germ cell development (gametogenesis-essential for genomic imprinting), where epigenetic reprogramming of DNA methylation rearranges the pattern parental imprinting and re-establishes totipotency to the gametes, while the second cycle of DNA methylation reprogramming occurs on fertilization (pre-implantation stage-essential for embryonic development), resulting in an asymmetric difference between the two parental genomes (Santos \& Dean, 2004; Biermann \& Steger, 2007).

\subsubsection{Epigenetic reprogramming during gametogenesis}

Recent studies have established that the same parents can produce epigenetically different gametes, DNA methylation profiles of which significantly vary across sperm and oocytes derived from the same individuals (Blewitt et al., 2006; Flanagan et al., 2006). Flanagan and coauthors mapped the methylated cytosine bases in the CpG islands of six 
disease-linked genes in human sperm (breast cancer tumor suppressor genes $B R C A 1$ and $B R C A 2$, brain development and function protein coding genes PSEN1 and PSEN2, Myotonic dystrophy type 1 gene DM1, and Huntington's disease gene, $H D$ ) and found that each sperm cell tested had a unique methylation profile. The functional impact that each modified and unmodified cytosine position has, still remains elusive. However the authors concluded that the epigenetic variability of the germ cells bestows diverse epigenetic initiation for offspring originated from the same parents (Flanagan et al., 2006).

\subsubsection{Epigenetic reprogramming during fertilisation}

A newly fertilized egg is totipotent as it differentiates into all embryonic tissues. Epigenetic reprogramming is the process known to return the highly differentiated sperm and egg nuclei to this nascent state in the early embryo (Reik \& Dean, 2002). The exact timing of epigenetic reprogramming is vital as it guarantees that both female and male germ cells attain an epigenetic state before the differentiation of the mature oocytes and sperm cells, in which the new parental imprints are established (Santos \& Dean, 2004; Biermann \& Steger, 2007).

Figure 1.4.2 shows that during fertilisation, the two gametes (female and male) are not at the same stage of meiotic maturation. The female gamete is arrested in MII metaphase stage impeding the signal of meiosis completion. In the haploid male gamete the majority of histones are replaced by protamines. Consequently, for the two gametes to finally merge into a zygote, a wide-range remodelling is required that involves the de- 
condensation of genetic material and the nucleoprotamine exchange for nucleohistone. In mice, paternal-specific demethylation occurs as early as three hours and reaches completion within six hours of zygote formation, which is many hours ahead of the initiation of DNA replication. The results of a number of studies show that aberrant reprogramming before specification of germ line engages both somatic and germ cells and thus could easily lead to the inheritance of epigenetic traits (Santos \& Dean, 2004).

Santos and Dean state that additional epigenetic asymmetry can be seen during the establishment of the first two lineages at the blastocyst stage and that this event of differentiation creates the epigenetic characteristics that are essential for the formation of the somatic inner cell mass and the extraembryonic trophectoderm. The primordial germ cells (PGCs) have a methylation pattern that resembles the somatic, which is erased before their migration into the gonadal ridge. Demethylation of the paternally inherited DNA is usually accomplished within one day, signifying that the whole process is active, while demethylation of the maternally inherited DNA occurs more slowly by a passive process. After the primordial germ cells enter in the gonadal ridge, the embryonic global-wide methylation pattern is reestablished in a sex-specific manner by the de novo methylation (Biermann \& Steger, 2007; Davis et al., 2000; Obata \& Kono, 2002).

Even though repeated sequences, tissue-specific gene promoters and gene coding regions are all generally hypermethylated, the CpG sites in CpG islands are not, even though some of them (like imprinted genes or CpG islands on the inactive $\mathrm{X}$ chromosome) do undergo differential allelic methylation (Yoder et al., 1997). Imprints in the female germ line have been 
found to re-establish in growing oocytes. Imprinting in the male germ line still remains elusive, even though it is believed to establish in the gonocytes (spermatogonia and oogonia). It is interesting to note that new data show differences in both timing and extent of methylation among different imprinted genes (Li et al., 2004). It is well know that subsequent to fertilization, the zygote undergoes epigenetic reprogramming. The question raised here is whether some germ-cell-specific epigenetic differences are able to survive the massive epigenetic reprogramming that occurs. The results of a recent study on the mouse agouti viable yellow $\left(A^{v y}\right)$ and axin fused $\left(A x i n 1^{F u}\right)$ alleles show that the abovementioned epigenetic differences persist during the transient loss of the parental germ line-profile of DNA methylation after fertilization (Blewitt et al., 2006).

Another example of epigenome surviving epigenetic reprogramming comes from cloned animals that encompass substantial epigenetic and phenotypic disparity from their single parent, even though this should not be the case as their DNA sequence is the same. Rideout and co-authors suggested that the observed epigenetic differences can be attributed to the remnants of the dissimilar epigenetic signatures; one being natural (parental) and one being highly artificial (clone). This would not be the case if the epigenetic profile of the parent was entirely erased and in its place a new epigenetic profile was established (Rideout et al., 2001). Last but not least, Kaminsky and co-authors found that the DNA methylation profiles of monozygotic twins are more similar than that of the dizygotic twins, which leads to the conclusion that zygotic epigenetic signals survive, even if partly (Kaminsky et al., 2009). 
Figure 1.4.2. Gamete methylation at meiotic stages.

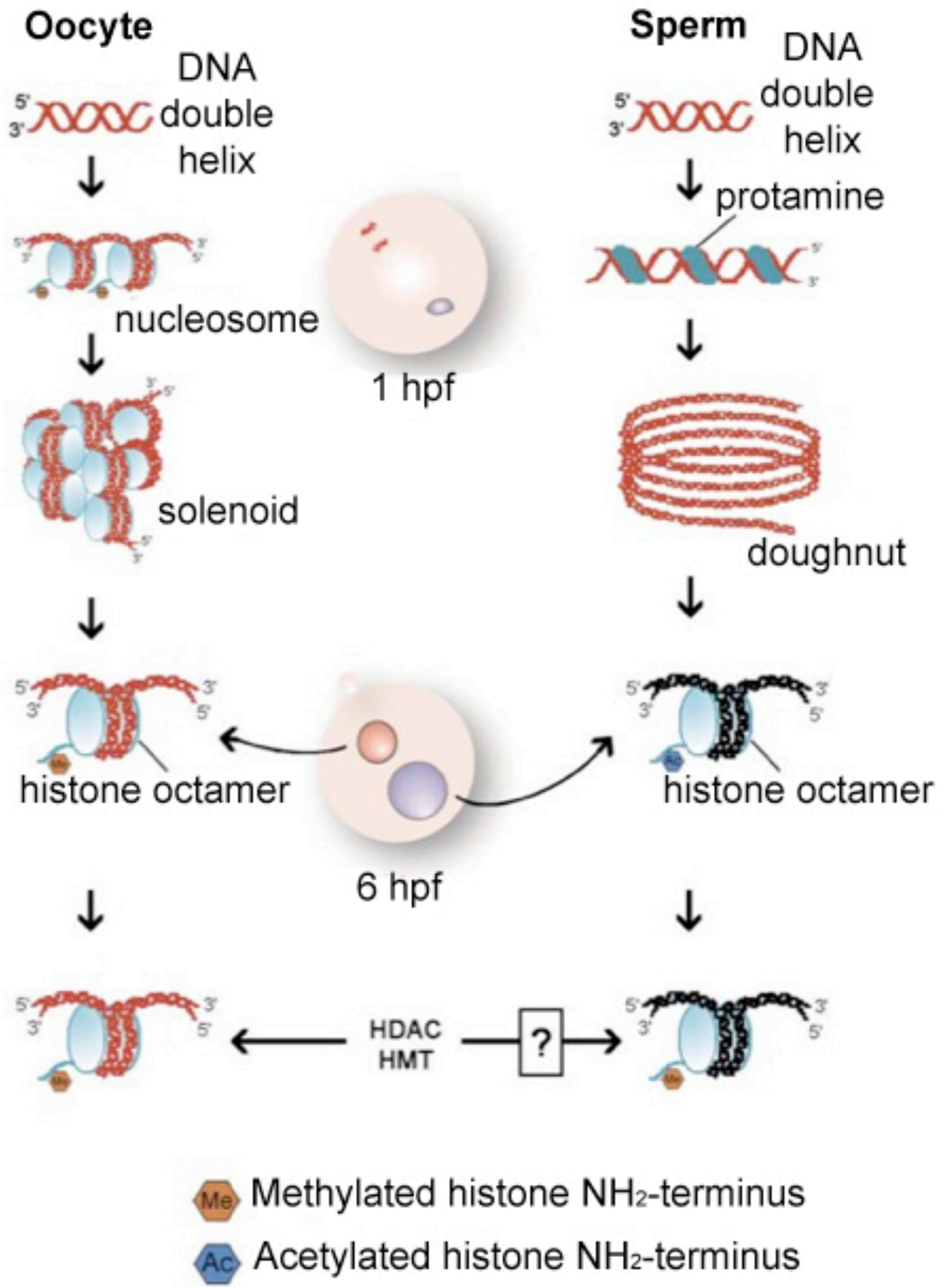

Taken from Santos \& Dean, 2004. 


\subsubsection{Evidence for histone domains retained in sperm}

The majority of histones are replaced by protamines in the mature spermatozoon, but this process is known to be incomplete, at least in some mammalian species. Western blot analysis of total acid extracts has revealed that both core histones (Gatewood et al., 1987; Zalenskaya et al., 2000) and many testis-specific histone variants (TH2B, $\mathrm{H} 2 \mathrm{AX}$ and $\mathrm{H} 3.3$ ) are all detected in human spermatozoa (Li et al., 2006; Van der Heijden et al., 2008) and as some reports now indicate, these histones are localised to the annular region of the human sperm nucleus (Li et al., 2008).

Core histones and histone bound DNA are also reported retained in a peripheral location in the mouse sperm nuclei (Pittoggi et al., 1999; Solov'eva et al., 2004), therefore nucleosomes are certainly not completely removed from mature ejaculate spermatozoa, even though estimations of their contribution to sperm chromatin range from $1 \%$ in the mouse (Balhorn et al. 1977 ), to $15 \%$ in the human (Gatewood et al. 1990) and over $50 \%$ in some marsupial species (Soon et al. 1997).

As state Miller and co-authors, these widely varying contributions imply that histone retention (if it has some important function) is not likely to be linked to a strictly enforced stoichiometry between nucleoprotamine and nucleosomes in sperm nuclei. And yet, the very existence of speciesdependent stoichiometric differences strongly suggests some function. The question on what that function could be and whether it could be linked with the transgenerational passing of information from the sperm to the offspring remains to be discovered (Miller et al.; 2010). 


\subsubsection{Transgenerational instability}

Transgenerational epigenetic effects are regarded as effects on the phenotype (or on patterns of gene expression) that are detected across more than one generation and that cannot be explained by Mendelian genetics or changes to the primary DNA sequence (Daxinger \& Whitelaw, 2012).

As mentioned above, during embryonic development, primordial germ cells migrate down the genital ridge and colonize the indifferent bipotential gonad before sex determination. While this occurs, their genomic DNA begins demethylation so that when germ cells undergo sex determination, their genome is unmethylated. At the beginning of sex determination, germ cell DNA undergoes re-methylation in a sex specific manner. Somatic cells in the developing gonads are essential for normal germ cell development and DNA methylation. Even though most of the genome undergoes demethylation and remethylation after fertilization and at the stage of early embryonic development, a small subset of genes are 'imprinted' and transmit a methylation pattern, which is unique to the following generations through the male or female germ-line (Chang et al., 2008).

Genomic imprinting is an epigenetic process, in which sex-linked marks in specific chromosome regions called imprints are expressed monoallelically; in a parent of origin-reliant manner (imprinted genes are expressed either only from the allele inherited from the mother or from the allele inherited from the father). Methylation and histone modifications are necessary for genomic imprinting to occur without alterations in the genetic sequence, and these epigenetic marks are established in the germline and 
maintained throughout all somatic cells of an organism (Biermann \& Steger, 2007; Wilkinson et al., 2007).

Numerous studies have recorded that when imprinted genes undergo alterations and modifications in their DNA methylation patterns, disease states are induced, thus changes in the epigenetic programming such as DNA methylation during the period of methylation pattern establishment in the germ-line can induce epigenetic transgenerational disease states. The current observations show that this transgenerational signal is transferred through the male germ-line to the subsequent generations suggesting that an epigenetic reprogramming of the germ-line is possible. The ability of an environmental factor to alter the epigenetic background of individual and cause transgenerational instability to all subsequent progeny has significant impacts on disease aetiology (Chang et al., 2008).

Irradiation was the first factor to be recorded causing DNA mutations and genomic instability leading to elevation in the mutation rates of the germline of multiple generations linked with tumor prevalence. Dubrova and co-authors examined the possible mechanisms of transgenerational instability documented in the progeny of irradiated parents and concluded that parental exposure to ionising radiation generates a signal in the parental germline that has the ability to transmit via a single sperm to the first generation, in which instability manifests after many cell generations later and can be detected in the developing germ line (Dubrova et al., 2000b).

Scientific evidence led to the hypothesis that this signal is DNA dependent and that it is inherited to the subsequent generations via an epigenetic mechanism. Furthermore this transgenerational signal is believed 
to be more likely to manifest through changes in DNA methylation, as methylation is transmissible through many cell divisions and could possibly influence the activity of DNA-repair systems. In conclusion, it is believed that DNA damage in the parental germ line triggers an epigenetic signal that survives the reprogramming of DNA methylation and early stages of development causing transgenerational instability, which is conserved for two subsequent generations at least (Dubrova et al., 2000a; Dubrova et al., 2000b).

Barber and co-authors investigated transgenerational instability in the progeny of directly irradiated male mice and found a constant preservation of global genomic transgenerational destabilisation in both subsequent generations $\left(F_{1}\right.$ and $\left.F_{2}\right)$, with mutation rates as high as those found in the directly exposed fathers. The study revealed prolonged, non-Mendelian transgenerational instability all through to the $F_{2}$ generation, ruling out the likelihood of specific genes in the exposed males driving this phenomenon (Barber et al., 2002).

Dubrova reviewed the findings of previous studies including the above and concluded that elevated mutation rates in all tissues tested (brain, bone marrow, spleen and germ line) were manifested in the progeny of directly irradiated parents, with mutation rates similar to the exposed parents and significantly higher than control groups. These findings raise a major issue regarding genetic risk assessments for potential cancer predisposition in humans exposed to ionising radiation and other biological stressors (Dubrova, 2003). 
Barber and co-authors later investigated the transgenerationally induced mutation rates and DNA damage in the somatic and germline tissue of the progeny of two different strains of irradiated mice (CBA/Ca and $B A L B / c)$ in an effort to elucidate the mechanism by which transgenerational instability is manifested. The data revealed statistically significant elevation in mutation rates of both protein coding regions and regions of hyper-variable tandem arrays (ESTRs) in the progeny of both strains, that was credited in endogenous damages caused by single and double-stranded DNA damages (Barber et al., 2006a; Barber et al., 2006b).

A study exposing male and female animals to ionizing radiation in utero revealed that the mutation frequencies in both the germline and somatic tissues of directly exposed males and females were similarly elevated. When tissues from the offspring of the directly irradiated animals were investigated, it was revealed that in contrast to paternal irradiation, maternal exposure did not affect the genomic stability of their offspring. It is possible that even though the epigenetic marks of foetal irradiation can survive developmental reprogramming in both males and females, and lead to genomic instability during adulthood, the failure of irradiated females to pass an instability signal to their offspring to be associated with early postfertilisation events (Barber et al., 2009).

As mentioned above, the paternal genome undergoes active DNA demethylation and other epigenetic changes within four hours of fertilisation, whereas this process is passive over several cell divisions in the maternal genome (Santos et al., 2002; Morgan et al., 2005). These data imply that the passive erasure of epigenetic marks in the maternal genome can diminish 
the transgenerational effects of foetal irradiation, while the active demethylation of the paternal genome may preserve more epigenetic modifications than the passive process occurring in the maternal pronucleus (Barber et al., 2009). This hypothesis is consistent with the results of several other studies showing transgenerational effects of prenatal exposure to the endocrine disruptor vinclozolin, where transmission was observed exclusively through the male germline (Anway et al., 2005; Nilsson et al., 2008).

To date, there is very strong evidence for transgenerational effects following paternal exposure to mutagens such as IR, chemicals and anticancer drugs (Barber et al., 2006a; Barber et al., 2009; Dubrova et al., 2000b; Dubrova et al., 2008; Glen et al., 2008; Glen \& Dubrova 2012; Vilarino-Guell et al., 2003) but the role of several non-mutagens that have been linked to genomic instability has not been thoroughly investigated and remains comparatively indeterminate.

The finding that the environment can influence the establishment of the epigenome, as well as the finding that a few epigenetic marks escape reprogramming between generations, increase the likelihood that environmentally induced epigenetic marks can be passed on to the next generation. If this is the case, it will profoundly change our understanding of inheritance (Daxinger \& Whitelaw, 2012). Investigating whether methyl deficient diet can potentially lead to transgenerational instability was one of the aims of this project. The question raised in this study was whether an 8week of methyl deprivation in directly treated male mice could lead to transgenerational instability in their progeny. 


\subsection{Mutation Detection}

Human population studies are limiting when interpreting data due to a considerable number of implications (Kendler \& Baker, 2007; Petronis, 2010), thus analyzing folate deficiency-related mutation induction in rodents is till this day the major source of information, investigating the role of folate to genome stability and the implicated genetic risk from folic deprivation in humans. There is a lot of conflicting in vivo evidence for mutation induction by folate deficiency in humans, thus model organisms have been extensively employed to shed some light where the population studies have failed.

Given that humans share extensive similarities with mice in numerous biological aspects, mice have been used as a model organism extensively over a long period of time. The use of mice reduces the degree of complication as all variables can be controlled in the laboratory; population studies are genetically homogeneous and all animals are exposed to the same environmental conditions to ensure that all organisms analysed are of the same age, developed in the same environment and given identical experimental treatments. When such homogeneity can be achieved, genetic differences can be ruled out, aiding data interpretation and variability of different environmental factors can be controlled. This is a clear advantage over human epidemiological studies, which will always have a degree of variation in one or more of these factors and thus attach a degree of difficulty and uncertainty when interpreting data (Dubrova 2005a).

Last but not least, the fast generation time of mice and their big litter numbers make it feasible to conduct transgenerational studies in a relatively short time frame. For all the above mentioned reasons, the mouse model has 
been chosen and employed in the making of this experiment.

As the effects of diet and folic acid in particular is one of the rising issues in the field of epigenetic therapy, a need for more efficient and sensitive methods for assessing its effects in genome stability is fundamental. Accuracy and minimum animal usage should be the key of any such new methods (Dubrova 2005a).

\subsubsection{Traditional approaches}

The majority of data on mutation induction in mice have been generated using pedigree-based methods such as (a) the Russell 7-locus test or (b) the dominant lethal test, both of which are based on mutation scoring among the offspring of control and exposed parents (Dubrova, 2005a). The Russell 7-locus test in mice detects mutations at a specific set of genes, usually genes that affect colour. The dominant lethal test is used for detecting mutations that cause embryonic death (Searle, 1974).

These two tests have been extensively used in the past and have provided substantial information on mutation induction during the previous decades. Their major disadvantage though is that they require an enormous number of animals (which increasingly becomes unacceptable in a bioethicpracticing scientific world), and on top of that these methods are highly time consuming. Limitation to the number of animal usage is now a necessity more than a matter of individual scientific judgment. Equally important, the two tests have been found insufficient (not sensitive enough) to detect mutation induction in low and intermediate doses of ionizing radiation and chemical mutagens. Since low and intermediate doses are mostly 
concerning the radiobiology and genetic toxicology researches (as it is usually the case of the amounts that human populations are most often exposed to), a need for a more sensitive and efficient test for the evaluation of mutation induction from different mutagenic agents is required. This technique should also be able to drastically reduce the number of animal usage (Searle, 1974; Dubrova, 2005a).

A new technique for mutation detection in the mouse germline was developed in 1993 by Dubrova and co-authors (Dubrova et al., 1993). It is based on mutation detection at the Expanded Simple Tandem Repeat (ESTR) DNA loci. The ESTR technique is highly sensitive on the analysis of mutation induction in the mouse germline caused by various mutagenic agents including ionizing radiation and chemical mutagens (Dubrova, 2005a).

\subsubsection{Repetitive DNA as markers of mutation rates}

Repetitive DNA loci are plentiful in most eukaryotic genomes and tend to be located in the non-coding genomic regions. They can also spontaneously mutate at relatively higher rates compared to protein-coding loci thus estimation of their mutation rates could be found to give a better representation of the genome in its entirety. Repetitive loci could therefore be good candidates for genomic mutation rate evaluation, even though not all of them are fitting for this purpose.

For example, interspersed repetitive loci SINEs (such as Alu elements) and LINEs (such as L1 elements) are known retrotransposons that incorporate into other genomic loci. However, their insertion rate is extremely low (one germline insertion per hundred individuals) in humans (Kazazian, 
1999), which makes them not ideal for mutation rate estimation. On the other hand, telomeres are also not suitable, since their sequence differs from humans to mice, thus preventing the reliable extrapolation of data from mouse models to humans (Wright \& Shay, 2000).

However, tandemly repeated sequences (such as ESTR loci) are widespread and in abundance in mammalian genomes. Additionally some of them tend to be unstable qualifying them as good candidates for mutation induction markers.

\subsubsection{Expanded simple tandem repeat (ESTR) loci}

Expanded simple tandem repeat (ESTR) DNA loci are long noncoding arrays of short repeat units (4-6bp) and represent the most unstable DNA loci in the mouse genome. ESTRs have a mutation rate that is more than a thousand times higher than normal protein coding genes providing the most sensitive tool ever developed for the investigation of heritable mutation induction in laboratory mice. The ability to robustly estimate mutation ESTR induction has dramatically reduced the number of animal usage as mutation detection can be recorded in fairly small samples (Yauk et al., 2002; Dubrova, 2005a; Somers, 2006).

The currient study focuses in the Ms6-hm ESTR locus, which is located on the chromosome 4, near the brown coat colour locus. The Ms6$h m$ ESTR locus consists of an extremely hyper-variable tandem array of the pentamer $d(C A G G G) n$ with a high somatic and germline mutation rate (of $10 \%$ per gamete). The pentamer $\mathrm{d}(\mathrm{CAGGG}) n$ has the ability to form intrastrand structures that is believed to be the reason of instability, which affects 
DNA replication, repair, and/or recombination (Weitzmann et al., 1998; Yauk et al., 2002).

The Ms6-hm pentameric repeat sequence can form intra-strand tetraplex strand specific structures that appear at the 3' end of the G-rich strand when four G-rich regions on a single strand interact to form a series of tetrads, which in turn form hollow stems that are bounded by loop formations created by the bases found in-between the G-rich regions. Sodium cations $\left(\mathrm{Na}^{+}\right)$were recorded to aid the stabilization of these formations effectively causing blockage of DNA synthesis (Weitzmann et al., 1998).

The size of the Ms6-hm array substantially varies between mouse strains. In the BALB/c inbred strain used in this study, the allele size is approximately $2.5 \mathrm{~kb}$ long ( 400 repeats).

\subsubsection{ESTR mutation induction}

The ESTR mutation mechanism has been thoroughly examined by Hardwick and co-authors and the results of this study provided strong evidence for the mechanisms involved in the spontaneous replication-driven mutations at the ESTR loci. Taking into account all these data along with results from previous publications, it becomes apparent that the spontaneously induced ESTR mutations are largely caused by replication slippage, which results in the generation of mutant alleles of different sizes due to gains or losses of repeat units. ESTR loci could thus be regarded as a class of expanded microsatellites (Hardwick et al., 2009).

As seen in Figure 1.5.1, ESTR mutation mechanism is believed to cause slippage during DNA replication. Slippage leads to the formation of 
new alleles with more or less repeat units depending on the strand containing the polymerase error (Yauk et al., 2002). Barber and co-authors found no correlation between germline mutation at repeat DNA and meiotic crossover in male mice exposed to X-rays or cisplatin. These observations lead to the hypothesis that meiotic recombination is not essential in the process of mutation (Barber et al., 2000).

Yauk and co-authors analysed the novel single molecules of spontaneous and radiation-induced mutation at a mouse tandem repeat locus after pre-meiotic X-ray exposure. The mutation frequencies in brain, sperm and spleen were recorded and it was found that somatic tissue did not show significant elevation. Specifically brain DNA had the lowest elevation in mutation frequency per progenitor molecule, which was attributed to the extremely low mitotic activity of the adult brain. Sperm on the other hand, had a significant increase in its mutation frequency, which was attributed to its huge number of ongoing cell divisions. Furthermore, the majority of mutations recorded were the result of small gains and losses (Yauk et al., 2002).

Fukuda and co-authors recorded that mutation in the ESTR regions is caused by formation of secondary structures in the structurally homogenous ESTR arrays that lead to replication problems such as replication stalling and replication slippage. Formation of quadruplex structures across rich GC regions of the Ms6-hm ESTR locus were verified in vitro and a UP1 DNA binging protein was found to be able to interact with the Ms6-hm locus, unwinding the formed quadruplex structures and restarting replication process. This study concluded that replication stalling is a major ESTR 
characteristic that could be the cause of replication slippage and mutation induction (Fukuda et al., 2002).

Dubrova reviewed the results of previous publications and summarized all found information on ESTR mutation induction (Dubrova, 2005b). The summary stated (a) that ESTR mutation rates in the germline of irradiated parents must be a result of damage induction in other parts of the genome, since the elevation in these loci was not caused by direct targeting, (b) other DNA repeat sequences showed similar elevations in mutation rates when directly exposed to radiation, thus direct exposure causes a global genome destabilization and damage including double strand breaks and chromosomal rearrangements, (c) the structure of ESTR mutants observed in all directly exposed, control mice, and their offspring is similar, thus one might assume that the mutation process in both groups is the same and that it does not involve the direct targeting of these loci after irradiation, (d) mutation induction in the ESTRs is believed to be the result of replication slippage. If this is the case it would be expected that the ESTR mutation rate will correlate with the number of cell division events in the observed tissue system. It should be stressed that to date the mechanisms of ESTR mutation induction remain poorly understood.

Numerous ionizing radiation and chemical mutagen studies have revealed that ESTR mutations occur mainly in premeiotic spermatogonia and stem cells. The average spermatogonial doubling dose (amount of radiation required to double the spontaneous mutation frequency) for low LET radiation is approximately 0.62-0.69 Gy (Dubrova, 2005; Somers, 2006), while average spermatogonia doubling dose for high LET radiation is 
approximately 0.18-0.34 Gy (Dubrova et al., 2000a; Somers, 2006). Chemical agents have also been found to cause significant ESTR mutation induction in premeiotic spermatogonia and stem cells, but have shown much less effect per unit dose than radiation. Previous studies report that ESTR mutation induction is in its maximum at low doses of radiation or chemical mutagens and could decrease at higher dose ranges (higher doses of mutagenic agents lead to higher rates of apoptosis which could explain the recorded phenomenon). Studies conducted on repair deficient mice (SCID and PARP-1 knock-outs) with spontaneous elevated levels of single and double strand breaks surprisingly showed no additional ESTR mutation induction following irradiation. On the other hand, p53 knockout mice showed an ESTR mutation induction identical to wild type mice (Burr et al., 2005; Somers, 2006).

Studies in mice in situ exposed to ambient air pollution reveal elevation in ESRT mutation frequencies in males exposed to high levels of particulate matter, and emphasize that ESTR assays can be used for the evaluation of environmental hazards under real-world conditions. As far as the mechanisms of ESTR mutation induction are concerned, so far they remain poorly understood, although the results of numerous studies have shown that they are clearly non-targeted (Dubrova et al., 1998; Vilarino-Guell et al., 2003; Somers, 2006).

Yauk and co-authors group investigated tandem repeat mutations, global DNA methylations, and the regulation of DNA methyltransferases in cultured mouse embryonic fibroblast cells chronically exposed to six different chemicals. The study confirmed that induced ESTR mutations arise through 
non-targeted mechanism. The authors reported that the changes in DNA methylation or in the pattern of DNA methyltrasnferase expression are linked to the indirect mechanism of mutation. Mutation induction in the mouse embryonic fibroblastic cells was found to vary between chronic exposures to different chemicals, from no increase to two-fold increase in mutation rates. The study recorded no direct link between ESTR mutation, global methylation or DNA methyltransferase transcription, but the majority of ESTR mutagens were recorded to cause changes in global methylation cell pattern. It was thus hypothesised that chemicals that have the ability to change the conformation of chromatin through changes in the methylation pattern may compromise the ability of mismatch repair enzymes to mend secondary structures that may form across ESTR loci resulting in mutation (Yauk et al., 2008).

As in any system of mutation induction, ESTR loci do have some limitations in their usage, such as the fact that since they are non-coding loci, it is not feasible (a) to directly compare their mutation frequencies to proteincoding genes (b) due to the fact that the mechanism of mutation in the ESTR loci is still unclear, extrapolating data to another genomic loci poses a challenge. Future studies should thus focus on the comparison of ESTR mutation induction with protein coding loci, refine dose responses of ESTR mutation induction, and develop ESTR markers for other animal species.

Having said that, a resent comparison of ESTR mutation rates with those from a protein-coding hypoxanthine guanine phosphoribosil transferase (HPRT) locus in the same spleenocytes, found similar increases in mutation rates at these loci (Barber et al., 2006). 
Figure 1.5.1. Replication errors.
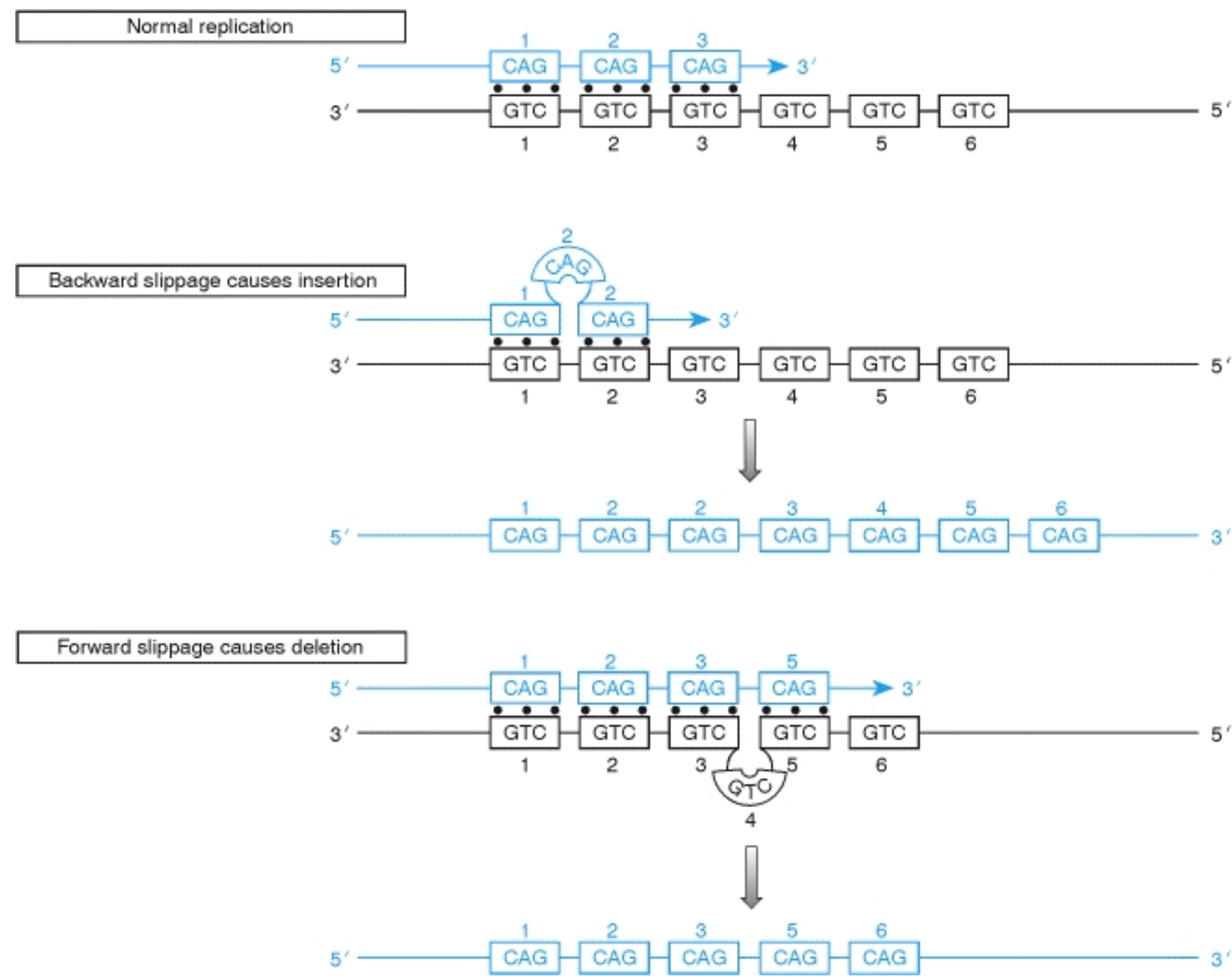

Taken from Strachan \& Read, 1999. 


\subsubsection{ESTR technique}

The ESTR technique utilizes the highly unstable expanded simple tandem repeat loci (ESTR). As previously mentioned, these ESTR loci consist of short repeats of four to six base pairs and have a highly spontaneous rate of mutation in the mouse germline (Bois et al., 1998).

The sensitivity of ESTR loci to radiation was compared with that of the loci of the Russell 7-locus test, and concluded that even though all loci have a very similar mutation induction mechanism, the ESTR system has proven to be substantially more sensitive than the Russell 7-locus test, as the changes in ESTR mutation rate are detectable at much lower doses of exposure (Table 1.5.1).

Table 1.5.1. Comparison of the sensitivity of different tests.

\begin{tabular}{|l|c|c|c|c|}
\hline & Dose range & & $\begin{array}{l}\text { Number of } \\
\text { mice }\end{array}$ & \\
\hline Exposure & 7-locus test & ESTR loci & 7-locus test & ESTR loci \\
\hline X-rays, & & & & \\
acute & $3-6.7$ Gy & $0.5-1 \mathrm{~Gy}$ & $1,051,869$ & 252 \\
\hline Y-rays, & $3-8.6 \mathrm{~Gy}$ & $0.5-1 \mathrm{~Gy}$ & 986,237 & 206 \\
chronic & & & & \\
\hline Fission & & & & \\
neutrons & & & & \\
\hline
\end{tabular}

Adapted from Dubrova, 2005a. 
The ESTR technique was then used for the analysis of ESTR mutation induction in the germline of male mice exposed to chemical mutagens including a very powerful germline mutagen called ethylnitrosourea (ENU). The study confirmed the sensitivity of the ESTR loci and its superiority to the Russell 7-locus test. Mutation induction in the ESTR loci was clearly detectable at doses as low as 12 milligrams per kilogram whereas the Russell 7-locus test was only able to detect significant increase in mutation rate for the doses of paternal exposure beginning at 50 milligrams per kilogram. As the main problem of the Russell 7locus test is the low mutation rates detected at its specific loci, a vast number of animals are required for the detection of significant increases in mutation rates (approximately thousands of mice). On the other hand, the ESTR technique robustly decreases the usage of animals (approximately a few hundred) as seen in Table 1.5.1. The table shows the difference in dose range and number of mice used at different exposures for the Russell 7 locus test and the ESTR loci test (Dubrova, 2005b).

Analysis of mutation induction at the ESTR loci level has been effective in providing valid predictions of the possible genetic risks of low and intermediate doses of radiation and mutagenic chemicals. It is now well established that ESTR loci have a significant advantage in recording induced mutations and have the ability to precisely predict the genetic risk of exposure to different mutagenic agents and any possible effects of transgenerational instability (Dubrova, 2005a).

The chosen method for monitoring DNA instability in this study is the single molecule PCR approach (SM-PCR) that provides unlimited access to the de novo mutants both in the germline and in somatic tissue. The method 
drastically reduces both the numbers of mice needed for the measurement of ESTR mutation rates (less than ten) and the experimental time. The SM PCR system for monitoring mutation was first described by Yauk and co-authors and it involves dilution of bulk genomic DNA samples to approximately single molecule concentrations. The ability to amplify vast numbers of molecules from a single individual allows robust estimations of somatic mutation rates and results of high precision and low standard deviation. The mutation rates of the unstable mouse ESTR locus Ms6-hm in all methyl depleted, methyl supplemented and offspring groups were estimated using the SM-PCR approach (Yauk et al., 2002).

\subsubsection{Tissue selection}

The decision on which tissue is best to analyze in the current study was based upon the findings of Barber and co-authors where the frequency of ESTR mutation was evaluated for the Ms6-hm locus of BALB/c male mice that were irradiated in utero or during adulthood. ESTR mutation frequencies were established in brain, bone marrow (BM) and sperm tissue (Barber et al., 2009).

Barber and co-authors recorded that in utero irradiation resulted in similar elevation in ESTR mutation frequencies across all tissues, and that was justified by the fact that the embryonic stage at which irradiation was performed corresponds to the period of maximum organogenesis where many of the foetal cells undergo active mitotic proliferation (Barber et al., 2009). In the brain tissue of the in utero irradiated males, highly elevated mutation frequencies were observed, whereas adult exposure did not alter the ESTR mutation rate observed in this tissue (Table 1.5.2). 
Table 1.5.2. ESTR mutation induction.

\begin{tabular}{|l|c|c|c|}
\hline & In utero & Adult (1week) & Adult (8 weeks) \\
\hline Brain & + & - & - \\
\hline Bone marrow & + & - & $+/-$ \\
\hline Sperm & + & - & + \\
\hline
\end{tabular}

* Data from Barber et al., 2009.

Given that the brain cells do not undergo mitotic proliferation in adults, these results clearly show that ESTR mutation induction only occurs in replication-proficient cells. In bone marrow (BM), irradiation of male mice as adults resulted in a relatively small change in ESTR mutation frequency, which may be explained by the fact that the percentage of actively dividing stem cells in mouse BM is very low (approximately $9.1 \times 10^{-5}$ cells in BALB/c mice) and that the DNA samples studies were enriched with genomes of nondividing supporting cells (Barber et al., 2009).

For sperm tissue, the data revealed no alterations in ESTR mutation frequency in the germline of male mice irradiated during adulthood and sacrificed 1 week after exposure, and that was justified by the fact that the samples assessed at this stage were derived from non-dividing mature sperm cells. In contrast, the frequency of ESTR mutation measured 8 weeks after adult irradiation is significantly elevated, which reflects the exposure of replication-proficient spermatogonia. These data imply that ESTR mutation induction in the male germline occurs mostly, if not exclusively, in replicationproficient mitotic cells (Barber et al., 2009).

Considering these data it appears that both spontaneous and induced ESTR mutations occur almost exclusively in replication-proficient cells and 
previous studies indicate that these mutations can be attributed to replication slippage (Barber et al., 2009).

Since sperm is the most actively dividing tissue in the mouse body, it was therefore chosen for analysis in both the $F_{0}$ and $F_{1}$ animals. Brain tissue was also analysed in the $F_{1}$ animals as a means of comparison between somatic and sperm tissue since prenatal neurogenesis is a mitotically proficient event. Cell division is mostly redundant in most areas of the adult postnatal brain, and as seen in Table 1.5.2 no ESTR mutation elevation would be detectable in the brain tissue of the directly exposed adult animals. Thus brain tissue was not analysed in the $\mathrm{F}_{0}$ animals.

\subsubsection{Mosaic and singleton mutations}

As in all mice breeding experiments, the offspring will inherit both a maternal and a paternal ESTR allele, and these wild-type non-mutant alleles will be found in all tissues of the offspring. However, if any of these maternal or paternal alleles happen to mutate in a certain tissue, then the new, unique and differentially sized allele would be detectable via electrophoresis on an agarose gel.

Any such mutated allele is named 'singleton' mutation and would have arisen via an autonomous mutation event. In the incidence where two or more ESTR mutants are found to have the same allelic size on an agarose gel, it becomes uncertain if these allelic mutations occur from the same mutation event or from independent mutation events (Hardwick et al., 2009). Clusters of two or more mutant alleles that share the same size are named 'mosaic' mutations (Favor \& Neuhäuser-Klaus, 1994) and as above- 
mentioned, it is not possible to identify if these mutations derive from a single mutation event or different independent mutations.

Minisatellite loci have heterogenous internal sequence structures, and their sequence subunits can be identified via the minisatellite variant repeat (MVR)-PCR technique, which employs specific primers for the different internal sequences, allowing the identification of the origin of mutation at these loci (Jeffreys, et al., 1991). On the contrary ESTRs have a homogenous internal structure that prohibits the use of multiple internal primers, thus mutants are classified either as sigletons or mosaic at the final stage of data analysis.

However, as already mentioned ESTRs are hypermutable with high spontaneous mutation frequencies, making the generation of two or more mutants of the same size highly probable. Therefore, in this mutation analysis, mosaic mutations were strictly considered to be those having five or more identical sized alleles in a given sample. It would be fair to assume that such alleles arose from a single mutation event that could even date back to the stages of early embryogenesis (Kelly et al., 1989; Kelly et al., 1991; Gibbs et al., 1993).

A new term named 'unique' mutations was therefore created, to include all singleton mutations and each cluster of mosaic alleles. With this design, five or more mosaic alleles of the same size would be counted as one unique mutation, as would one singleton mutation (Hardwick et al. 2009). 


\subsubsection{In vivo gene mutation assays}

Identifying genotoxic hazards in humans is vital for clinical trials and population safety during treatments. The current set of tests for identifying hazards, as agreed by U.S, E.U and Japan is a series of in vitro and in vivo genotoxicity assays. It is generally agreed though, that these set of tests are not ideal for the prediction of carcinogenicity, which is one of the principal applications of these assays to begin with. This could be explained by the fact that all the gene mutation assays that are part of the current tests (such as the bacterial reverse mutation and the mouse lymphoma assays) are in vitro tests, which are not able to replicate the metabolism and pharmacodynamics that occur in vivo (Miura et al., 2008).

The currently existing in vivo gene mutation assays have limited ability to serve as screening tests for hazard identification. A perfect screening test would be able to utilize high throughput methodology and would be able to detect mutations in a number of animal species, including humans. Currently the most frequently used mutation assays in vivo are the numerous bacterialtransgene rodent models such as the lacl or lacz, the lymphocyte hypoxanthine-guanine phosphoribosyltransferase (Hprt) assay, and the human Glycophorin A (GPA) assay (Miura et al., 2008; Dobrovolsky 2010b). A bulk of scientific data have been generated in the past using the transgenic models and the Hprt assay, however these assays are relatively expensive and time consuming and entail in vitro culturing for the identification of mutants and confirmation of the gene mutation. More over, even though the Hprt assay can be conducted in humans, the transgenic assays cannot (Dertinger et al., 2010; Dobrovolsky et al., 2010a).

Flow cytometry (FCM) could become a powerful tool when employed for 
rapid quantification of rare mutation events, even though this technology cannot be used in the Hprt and transgenic assays. On the other hand the GPA assays is able to assess mutagenicity using flow cytometry (FCM), but GPA assay has only been developed in humans and thus its usage is limited to the fifty percent of humans that are heterozygous for the target locus. The development of a new rapid and flexible assay for the measurement of in vivo gene mutation was thus required (Miura et al., 2008; Ohtani et al., 2012).

The specifications for a model sentinel gene for measuring spontaneous somatic mutations are (a) the gene must not be essential for growth or viability and (b) the mutant phenotype must be detectable among a vastly larger population of normal cells. As a single mutation with a loss of function could be complemented by the wild type allele on the homologous chromosome, most autosomal genes are classified unsuitable for this purpose. However, due to hemizygosity in males and X-inactivation in females, a single mutation is sufficient to inactivate X-linked genes.

\subsubsection{Pig-A assay}

A new approach first suggested by Araten and his group in 1999, in which mutation is detected by FCM in the endogenous phosphatidylinositol glycan complementation group of the X-linked gene A (Pig-A), shows very promising. Several characteristics of the Pig-A assay make it a favourable candidate for in vivo somatic cell mutation screening (Araten et al., 1999; Araten et al., 2010).

Pig-A encodes a subunit of a protein complex in the endoplasmic reticulum, which catalyzes the transfer of $\mathrm{N}$-acetylglucosamine to phosphatidylinositiol, the first reaction in the biosynthesis of gly- 
cosylphosphatidylinositol (GPI) cytoplasmic membrane anchors. These GPI anchors are structures that attach specific proteins to the cell surface of various types of cells (such as the hematopoitic cells) in a plethora of animal species, including rodents and humans. Mutation in the Pig-A gene could disrupt GPI synthesis, which would lead to the elimination of expression of proteins that entail it to attach to the cell surface such as CD24, CD52, CD55, CD59 and thus lead to a deficiency of GPI-anchored proteins (or markers). The lack of the anchor or GPI-anchored markers is detectable and quantifiable by using FCM (Phonethepswath et al., 2008; Aratena et al., 2010).

Studies have shown that a GPI-deficient phenotype occurs in patients with paroxysmal nocturnal hemoglobinuria $(\mathrm{PNH})$, and that the basis of this condition is a mutation of the PIG-A gene. Due to this condition, it is now known that a broad spectrum of mutations can disrupt the function of Pig-A. Even though numerous other genes apart from Pig-A are necessary for GPI synthesis, only the Pig-A gene is located on the $\mathrm{X}$ chromosome, so that a single mutation results in an altered cell phenotype; males are hemizygous for X-linked genes, and Pig-A is completely silenced on the inactivated $\mathrm{X}$ chromosome in females, therefore only one mutation is required to produce the GPI (-) phenotype, and males and females are equally affected (Aratena et al., 2010; Kimoto et al., 2011).

Bryce and his group in their 2008 study aimed to evaluate whether lack of GPI-anchored proteins could form the basis of an in vivo mutation assay in rats. They used a CD59-negative cell surface phenotype for the indication of Pig-a mutation, and employed fluorescents anti-CD59-PE, thiazole orange (for the identification of differentiate mature erythrocytes, reticulocytes, and 
leukocytes) and anti-CD61 (to resolve platelets). The experiments focused on two cell populations, (a) total erythrocytes and (b) RETs, and female rats were treated in three occasions with the model mutagens ENU (100 $\mathrm{mg} / \mathrm{kg} / \mathrm{day}$ ) or DMBA (40 mg/kg/day). Blood samples were collected at various intervals, as late as six weeks after the end of treatment. All collected data from week 4-6 showed significant and consistent increase in mutation frequencies for both ENU and DMBA treated animals, respectively. The assay also detected higher elevation in frequencies for RETs compared to total erythrocytes. The recorded data support the hypothesis that a competent in vivo mutation assay can be developed around flow cytometric recording of erythrocytes and/or RETs that exhibit aberrant GPI-anchored protein expression (Bryce et al., 2008).

Miura and his group in 2008 verified (a) that genotoxins do increase the frequency of GPI-anchored protein-deficient cells (GPI-deficient cells), and (b) that GPI-deficient cells in animal tissues are Pig-A mutants. With the confirmation of these results, the frequency of GPI-deficient cells in animal tissues showed that they can serve as a reporter of in vivo gene mutation. The group then designed conditions for the FCM identification of GPIdeficient cells among the peripheral red blood cells (RBCs) and spleen Tcells of rats and determined the spontaneous and induced frequencies of GPI-deficient cells in control and N-ethyl-N-nitrosourea (ENU)-treated rats. In this study, CD59 and CD48 (GPI-anchored cell surface markers) were used as reporters of Pig-A mutation in peripheral RBCs and spleen T-cells, respectively, as well as a fluorescent reagent that specifically binds to GPI anchors (FLAER), for the assessment of the frequency of GPI-deficient RBCs (Miura et al., 2008). 
The results from this study showed that the spontaneous and ENUinduced frequencies of GPI-defective cells assessed by flow cytometry (FCM) were similar for rat peripheral RBCs (CD59-negative cells) and spleen T-cells (CD48-negative cells). Since circulating red blood cells (RBCs) are not nucleated cells, while spleen T-cells are, these data suggest that mutations also occurred in the Pig-A gene of nucleated erythroid precursors and/or of hematopoietic stem cells. Furthermore the results showed there is an excellent correlation between the frequencies of CD48- negative rat spleen T-cells assessed by flow cytometry (FCM) and that of Pig-A gene mutants from a previous clonal selection assay. Based on their results, Miura et al. argued that it is possible that CD59- negative red blood cells (RBCs) detected by FCM also derive from Pig-A mutant nucleated cells, and that the frequency of CD59- negative RBCs reflects mutational events in the animals. They then concluded that the RBC FCM assay could be a useful tool for the identification of genotoxic hazard in a rapid and minimally invasive manner (Miura et al., 2008).

Dobrovolsky and his group in 2009 argued that the estimated frequencies of CD59-deficient RBCs that Miura and co-workers recorded in their 2008 study may have been artificially high due to the detection of nonspecific events as mutants. Such nonspecific events could be either debris or non-targeted cell populations (like cells other than RBCs) that have naturally low CD59 expression. Araten and co-authors in their 1999 paper reported that spontaneous Pig-A mutant red blood cells (RBCs) are seen at a frequency of approximately $1 \times 10^{-6}-1 \times 10^{-5}$ to wild-type cells. This frequency was found to be correlating with the results of a small human study that looked at mutant granulocytes and RBCs (Araten et al., 1999). Dobrovolsky 
states that such a low frequency of specific events creates a challenge to accurately identify mutations using FCM for detection, and inclusion of the so-called non-specific 'noise' is expected to have its maximum effects on mutant frequencies created by very weak mutagens and proportionately less impact on mutant frequencies produced by strong mutagens. All 'background noise' integrated in these events (which are recognized in the blank/controls as spontaneous mutants) will thus tend to reduce the mutation frequency ratio between the treated and control groups and hence, the sensitivity of the assay. To increase the sensitivity of the rat Pig-A red blood cell assay, Dobrovolsky and his group developed an alternate method for measuring CD59-negative (CD59neg) RBCs in which events are analyzed only if they are recognized by a rat erythroid-specific antibody (recognized as red blood cells), which dramatically reduces the possibility of scoring non-red blood cells as mutants (Dobrovolsky et al., 2009).

Araten and his group in 2010 employed the Pig-A assay for the direct measurement of the mutation rate $(\mu)$ of B-lymphoblastoid cell lines (BLCLs) in an effort to record whether hyper-mutability is necessary to account for the high frequency of mutations in cancer. They estimated that Pig-A's average value is $10.6 \times 10^{-7}$ mutations per cell division in the B-lymphoblastoid cell lines (BLCLs) from normal donors. Measuring the mutation rate $(\mu)$ in human malignancies they recorded elevation in $\mu$ in cell lines derived from $T$ cell acute lymphoblastic leukemia, mantle cell lymphoma, follicular lymphoma in transformed phase, and 2 plasma cell neoplasms. On the other hand, $\mu$ was found to be significantly lower in marginal zone lymphoma cell line and 5 other plasma cell neoplasms. The highest value measured in this study $\left(3286 \times 10^{-7}\right)$, was approximately two fold above the range we have observed 
in non-malignant human cells. Araten concluded that the type of genomic instability detected in this assay is a very common but not universal feature of hematologic malignancies (Araten et al., 2010).

Even though the Pig-A assay has many favourable characteristics, Pig-a mutations remain rare events. Due to this fact, many cells are needed for interrogation per analysis. Combining FCM with peripheral blood analysis is ideal for this purpose, as hundreds of thousands of cells may be scored in a short period of time. This methodology is very straight forward as it examines red blood cells (RBCs) for aberrant GPI anchor expression and when focusing on the youngest red blood cells (RBCs) like reticulocytes, this method has been shown to detect alterations in GPI anchor-mediated surface protein expression in as little as one week following in vivo exposure to chemical mutagens (Bryce et al., 2008).

As Bryce and co-authors state in theoir 2008 paper, there is significant potential for this assay to be used in a number of different areas such as pharmaceutical development and chemical safety assessment, where simple in vivo mutation assays could provide valuable toxicological data. The ability that this assay has to be incorporated into the standard toxicity studies means more efficient use of resources for required in vivo testing. Furthermore, since numerous studies in different mammalian species of toxicological interest record that the Pig-a gene is conserved them, this assay offers the potential to bridge the gap between preclinical studies and human clinical trials (Bryce et al., 2008).

As prior work with humans, rats and mice, suggests that the GPI anchor deficient phenotype is a reliable indicator of Pig-a mutation, my line of investigation will employ simplified blood handling procedures and the assay 
principle will be applied to the folate depleted and control Balb/c mice. With this method, blood is harvested and erythrocytes are isolated and incubated with PE-conjugated anti-CD24. Flow cytometric analysis quantifies GPI (CD24 negative) anchor-deficient erythrocytes in an effort to record all mutation events.

As you can see from Figure 1.5.2 below taken from Phonethepswath and co-authors 2008, events are recorded in three plots. The left plot represents a blood sample that contains both cells in which staining was blocked with a non-conjugated antibody, mixed with stained cells, so the two populations could be seen together. This plot is used as a calibration standard - sets the position of the vertical line that defines mutant versus non-mutant cells. The middle plot represents control mouse blood. As it can be seen in the plot, no mutant erythrocytes were detected in the control sample. On the right, the plot represents mutagen treated mouse blood, and elevated numbers of events (Pig-a mutant erythrocytes) are present in an approximation of a hundred mutant cells per million (Phonethepswath et al., 2008).

Figure 1.5.2. Pig-A assay
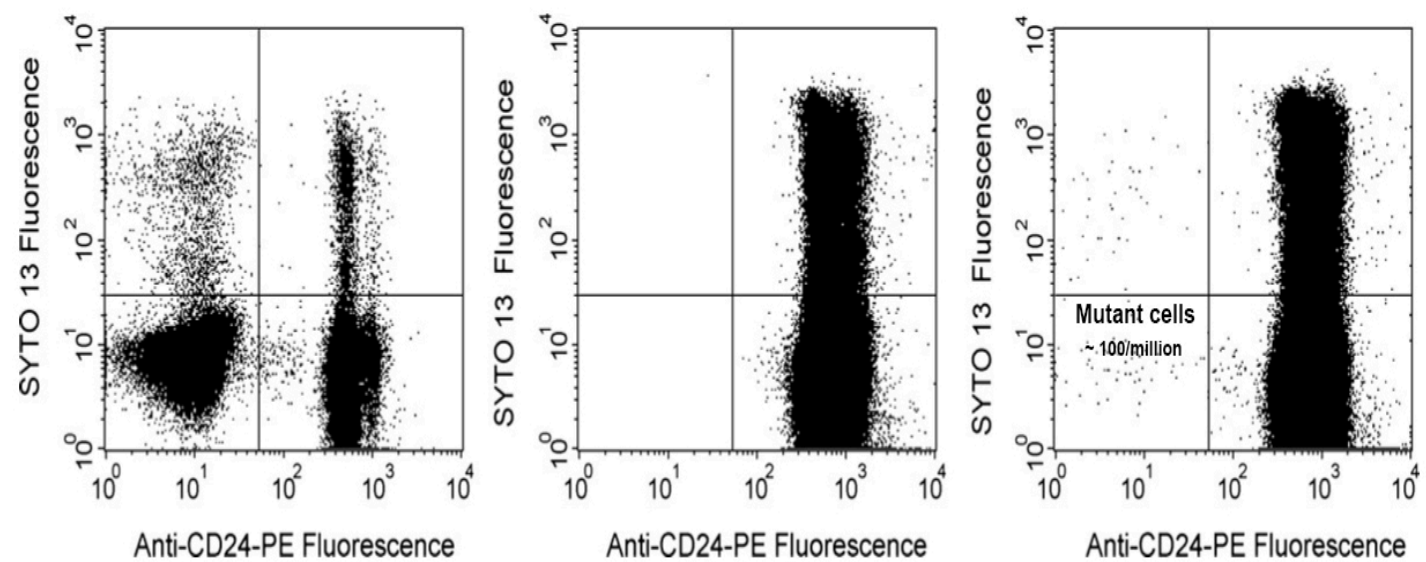

Figure 1.5.2 modified from Phonethepswath et al., 2008. 


\subsection{Aims and Objectives}

The effects of methyl-deficient diet on mutation induction:

To evaluate the effects of a methyl-deficient diet on mutation induction, the frequency of ESTR mutation was determined in male mice sacrificed at the end of treatment. To establish whether the effects of methyl-deficient diet can be reversed by the methyl-adequate diet, male mice were sampled over the period of time up to 10 weeks after the end of dietary restriction.

Given that ESTR mutation is a replication-based process involving polymerase slippage, there should be a relationship between the accumulation of mutation and mitotic index. If methyl-deficient diet results in the long-term genome destabilisation, then ongoing genomic instability should lead to the accumulation of mutations in tissues with a high mitotic index (spermatogonia, bone marrow). By profiling mice over a considerable period of time following treatment, the contribution of ongoing genomic instability to the dynamics of ESTR mutation accumulation can be established. This work aims to provide important information on the long-term changes in mutation rates and will allow the establishment of a time period over which they remain elevated in treated animals (Dubrova, 2005b; Barber et al., 2009; Shanks et al., 2008; Hardwick et al., 2009).

The effects of paternal methyl-deficient diet on transgenerational instability:

To establish whether paternal history of a low dietary folate intake could result in transgenerational changes in ESTR mutation rates, treated males were mated to control females. Tissue samples were taken from a number of eight-week-old males, fathered by males that have been either 
treated with control or methyl-deficient diets. Using the experimental approaches mentioned above, ESTR mutation frequencies were established in DNA samples extracted from sperm and brain of the offspring of treated and control males. Such analysis aimed to provide important information on the epigenetic effects of a low dietary folate intake on transgenerational instability.

The effects of methyl-donor deficient diet on ESTR mutation spectrum:

To establish whether methyl-donor deficiency alters the spectra of ESTR mutation in the mouse germline, the germline length changes were defined for all unique ESTR mutations found in sperm and brain of all control and treated males, as well as in their offspring. Such analysis will give an inside in to the mechanisms involved in mutation induction.

Pig-A assay:

To evaluate the effects of a methyl-deficient diet on mutation induction in the Pig-A protein-coding gene, that directly affects the occurrence of the GPI anchor on the surface of RBCs. This pilot study will aim to validate the results obtained by the ESTR assay, and provide a broader insight on the effect of the methyl-deficient diet on both end-points. 


\section{Chapter 2. Materials and methods}

\subsection{Materials}

\subsubsection{Animals}

Forty 4-week old male BALB/c mice were obtained from Harlan (Bicester, UK) and housed at the Division of Biomedical Services, University of Leicester. Shortly after their arrival, animals were split in two groups. The first group (test group) was fed on a choline and folic acid-deficient diet (see Appendix 5.2-Diets: Dyets D518892), while the second (control group) was fed on a similar diet containing normal levels of choline and folic acid (see Appendix 5.2-Diets: Dyets D518894). The two diets were the same, apart from the fact that one contained choline $(14.48 \mathrm{~g} / \mathrm{kg})$ and folic acid $(2 \mathrm{mg} / \mathrm{kg})$, while both those components were absent from the other. Both diets were low in methionine $(0.18 \%)$.

All $F_{1}$ generation mice were fed exclusively with control diet and were culled at eight weeks of age. Animals were weighed and examined weekly by DBS staff. All animal procedures were carried out under the Home Office project license No. PPL 80/1564.

\subsubsection{Experimental design}

To establish the effects of dietary methyl-donor deficiency on mutation induction in the germline of treated animals, the frequencies of ESTR mutation in DNA samples extracted from sperm were established in three groups of exposed males and compared with those in age-matched control males maintained on the control diet (Table 2.1). 
Table 2.1. Experimental design.

\begin{tabular}{lclcc}
\hline & & & \multicolumn{2}{c}{ No analysed } \\
Group & Age, weeks & Tissue & Control & Test \\
\hline$F_{0}$, Group A & 12 & Sperm & 4 & 4 \\
$F_{0}$, Group B & 18 & Sperm & 4 & 4 \\
$F_{0}$, Group C & 22 & Sperm & 4 & 4 \\
$F_{1}$, Group B & 8 & Sperm, brain & 4 & 4 \\
$F_{1}$, Group C & 8 & Sperm, brain & 4 & 4 \\
\hline
\end{tabular}

Table shows the age of animals included in the study, their numbers, and tissues taken from them.

The first group tested was $F_{0}$ of Group $A\left(\right.$ Test $F_{0} A$ ) in which animals were treated with the methyl-deficient diet for 8 weeks and then sacrificed immediately after treatment.

The frequency of ESTR mutation in this group was compared with its age-matched control group (Control $\mathrm{F}_{0} \mathrm{~A}$ ).

To establish whether the effects of a methyl-deficient diet can be reversed by the methyl-adequate diet, male mice were also sampled 6 (Test $\left.F_{0} B\right)$ and $10\left(\right.$ Test $\left.F_{0} C\right)$ weeks after the end of dietary restriction, and their ESTR mutation frequencies were compared with those in the age-matched control groups (Control $\mathrm{F}_{0} \mathrm{~B}$ and Control $\mathrm{F}_{0} \mathrm{C}$, respectively). 


\section{Figure 2.1. Experimental design.}

The flow diagram represents the design of this experiment and shows all the studied groups and the time of sampling.

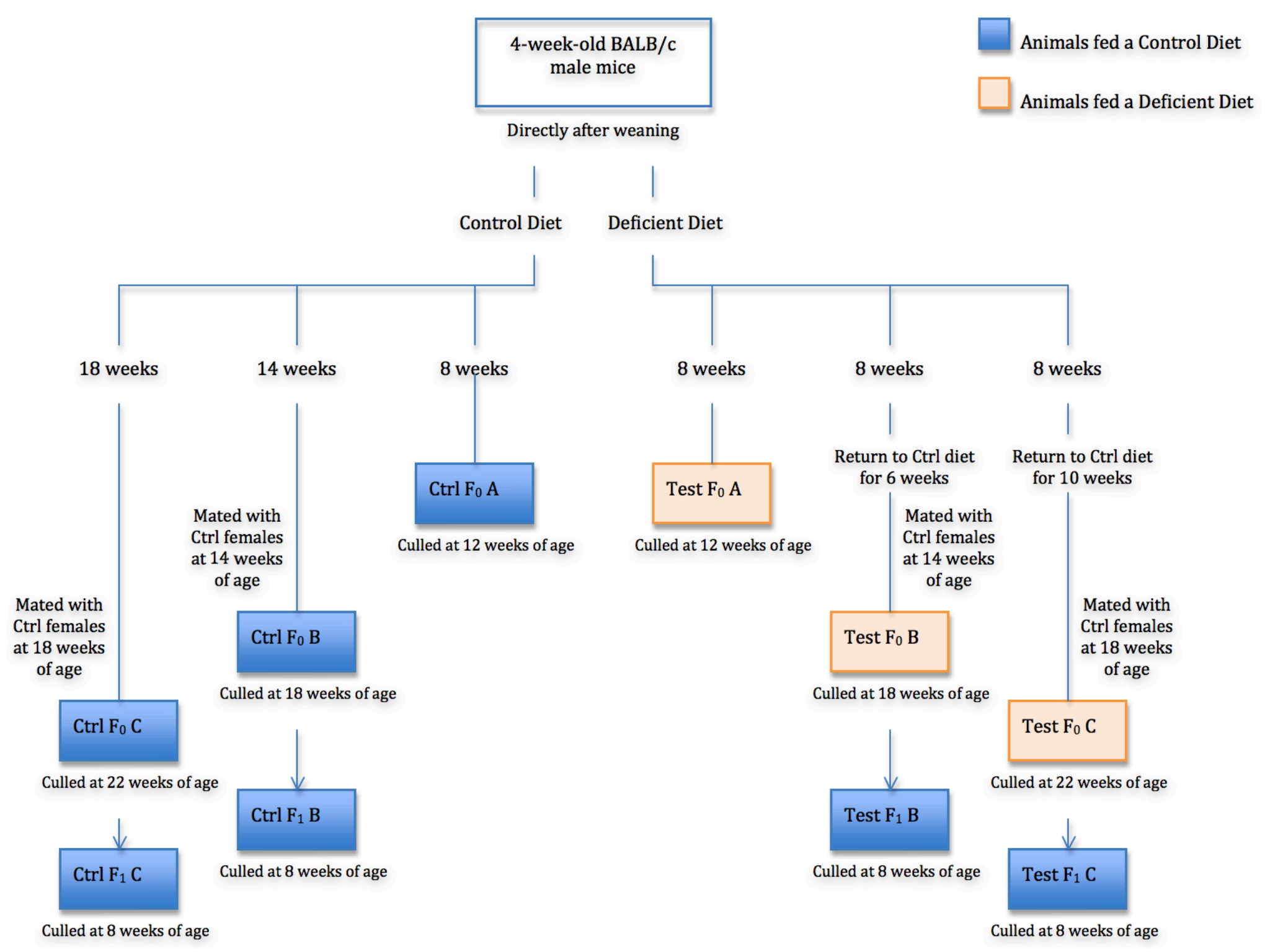


To establish whether paternal history of a low dietary methyl-donor intake could result in transgenerational instability, males from group B were mated to control females ( 2 females per male) two weeks after the end of their dietary restriction (Test $F_{1} B$ ) and males from group $C$ were mated to control females ( 2 females per male) six weeks after the end of their dietary restriction (Test $\left.F_{1} C\right)$. Successfully mated females were identified by the appearance of the vaginal plug.

In both groups, the frequency of ESTR mutation was established in DNA samples extracted from both sperm and brain tissues of eight-week-old male $F_{1}$ offspring (Figure 2.1).

Male germ cells were specifically targeted in order to determine the impact of methyl-donor dietary restriction on mutation induction in mice, as according to the results of previous studies, ESTR mutation induction mostly occurs in replication proficient tissues such as mitotically-active spermatogonia (Dubrova et al., 1998; Barber et al., 2000; Dubrova et al., 2000; Vilarino-Guell et al., 2003).

As male mice were sacrificed at least 8 weeks after the beginning of dietary intervention, the frequency of ESTR mutation was therefore measured in sperm cells derived from exposed mitotic $A_{s}$ spermatogonia (Searle, 1974). 


\subsubsection{Single Molecule PCR technique}

\subsubsection{Chemical and molecular biology reagents}

Chemical reagents were supplied by Cambrex Bio Sciences (Rockland, USA), Fisher Scientific (Loughborough, UK), Flowgen (Ashby de la Zouch, UK), FMC Bioproducts (Rochland, USA) and Sigma-Aldrich Company Ltd. (Poole, UK).

Molecular biology reagents where purchased from ABgene (Epsom, UK), Cambrex Bio Science (Rockland, USA), Healthcare UK Ltd (Chalfont, UK), GE Healthcare UK Ltd. (Chalfont, UK), Invitrogen (Paisley, UK), New England Biolabs (Hitchin, UK), Promega (Southampton, UK), Roche Molecular Biochemicals (USA), Sigma-Aldrich Company Ltd (Poole, UK) and United States Biochemical Corp (USB) (Cleveland, USA).

\subsubsection{Radiochemical reagents}

Radiochemical reagent $\alpha-{ }^{32} \mathrm{P}-\mathrm{dCTP}$ was purchased from PerkinElmer (Austria).

\subsubsection{Enzymes}

Protease $\mathrm{K}$ used for DNA extraction was obtained from Sigma-Aldrich (Poole, UK), restriction endonuclease Mse1 was supplied from New England Biolabs (NEB) (Hitchin, UK), Klenow enzyme (synthetic derivative of DNA polymerase 1 of E.coli) was purchased from United States Biochemical Corp (USB) (Cleveland, USA) and high fidelity thermo-stable PCR Taq and Tgo DNA polymerases where purchased from Roche Ltd (Hertfordshire, UK). 


\subsubsection{Oligonucleotides}

Primers and probes used for the amplification of sample DNA where purchased from Sigma-Aldrich (Poole, UK).

\subsubsection{Molecular weight markers}

The $1 \mathrm{~kb}$ DNA ladder was purchased from Invitrogen UK (Paisley, UK) and the 200 bp DNA ladder was purchased from Promega (Southampton, UK).

\subsubsection{Standard Solutions}

All standard solutions used in this experiment $10 \times$ tris-borate/EDTA (TBE) electrophoresis buffer, $20 \times$ sodium chloride sodium-citrate (SSC) buffer, depurinating solution, denaturing solution and neutralizing solution where produced by the media kitchen of the department of genetics, university of Leicester, as described by Sambrook and Russell (Sambrook \& Russell, 2001).

\subsubsection{Equipment}

Equipment used for the purposes of this experiment where supplied from Bio-Rad (Hemel Hempstead, UK), Eppendorf (Hamburg, Germany), Fisher Scientific (Loughborough, UK), Genetic Research Instrumentation (Braintree, UK), Heraeus Instruments (Hanau, Germany), NanoDrop Technologies(Ringmer UK), Purite Ltd (Oxon, UK), Qiagen(Crawley, UK), ThermoScientific (Ashford, UK), and Ultra Violet Products Life Sciences (Cambridge, UK). 


\subsubsection{Computer software}

Microsoft Office Word 2008 (version 12.2.0) and Adobe Photoshop CS4 were used for the purposes of this write up. Microsoft Office Excel 2008 (version 12.2.0) was used to record the data obtained from the SM PCR optimizations and Poisson analysis. The computer software used for assessing optimization results was created by Professor Y.E Dubrova using BASIC. Programs STATISTICA (version 6) and SYSTAT (version10) were used for mutation frequency analysis.

\subsubsection{Statistical analysis}

ESTR mutation frequencies, 95\% confidence intervals and standard errors were assets using a modified approach proposed by Chakraborty and co-workers (Zheng et al., 2000). The number of amplifiable molecules for each Poisson reaction was estimated as $N_{i j}=\lambda_{i j} \times 96$, where $\lambda_{i j}$ is the number of amplifiable molecules per PCR reaction $j$ and animal $i$. The frequency of ESTR mutation per group was estimated as $f=m / \Sigma \Sigma N_{i j}$, where $m$ is the total number of mutation found in this group. The standard errors of mutation frequency were estimated as $\left.\mathrm{se}_{f}=f^{*} \sqrt{ }\left(\mathrm{se}_{N} / N\right)^{2}+\left(s \mathrm{e}_{m} / m\right)^{2}\right)$

Power calculations for this project are based on the Poisson approximation (see IAEA, 1991 for details. IAEA. The International Chernobyl Project. Technical Report. IAEA, Vienna, 1991). The expected number of mutations in the exposed and control groups can be estimated as $M_{1}=m_{1}$ * $N_{1}$ and $M_{0}=m_{0} * N_{0}$. Given that standard deviation for the difference between the two groups, $D=M_{1}-M_{0}$ is $s d=\sqrt{ }\left(M_{1}+M_{0}\right)$, the statistical power can be estimated solving the equation $D / s d>2$. 


\subsubsection{Pig-A assay}

\subsubsection{Chemicals}

Lympholyte mammalian cell separation reagent was purchased from Sigma-Aldrich Company Ltd. (Poole, UK). Foetal bovine serum was purchased from Invitrogen (California, USA) AND PE conjugated anti- antiCD24-PE was purchase from BD Biosciences (California, USA).

\subsubsection{Materials}

$25 \mathrm{G}(0,5 \times 16 \mathrm{~mm})$ gauge needles and syringes were purchased from BD Biosciences (California, USA). 15mL heparinised tubes were purchased from Sarstedt Ltd (Leicester, UK). 15mL conical polypropylene tubes were purchased from Sarstedt Ltd (Leicester, UK).

\subsubsection{Equipment}

Eppendorf centrifuge 5804 used for the purposes of this experiment was purchased from Eppendorf (Hamburg, Germany) and BD FACSCanto ${ }^{\text {TM }}$ II Image Flow Cytometer was purchased from Becton Dickinson and Company (Oxfordshire, UK).

\subsubsection{Data analysis}

All experimental data from the Pig-A assay were analysed using a BD FACSDiva (version 6.1.3) software package. Statistical analysis of the Pig-A data was performed using STATISTICA (version 6) and SYSTAT (version10) software. 


\subsection{Methods}

\subsubsection{Single molecule PCR technique}

\subsubsection{Tissue collection}

All mice were marked with ear punches to permit the identification of an individual mouse, and were culled via exsanguinations following a lethal dose of pentobarbital. Bone marrow, brain, caudal epididymis, heart, kidney, liver, spleen, and tail samples were taken using sterilized scissors and tweezers. All tissues were collected in eppendorf tubes and kept on ice. Blood was stored in eppendorf tubes containing clotting inhibitor beads (EDTA) and was always used on the day of extraction. Bone marrow specimens were collected using a syringe to pass $500 \mu$ l of phosphate-buffer solution (PBS) through each tibia and into an eppendorf tube. Brain, liver, spleen and kidney tissues were snap frozen in liquid nitrogen. At the end of tissue collection all tissues were kept in $-80^{\circ} \mathrm{C}$ freezers until required. All mice procedures were carried out under guidance issued by the Medical Research Council in 'Responsibility in the use of animals for medical research' (July 1993).

\subsubsection{DNA extractions}

\subsection{Brain DNA extraction}

All DNA extractions were performed in a Category II flow hood (Walker Safety Cabinets Ltd, Glossop, UK) to minimize risk of contamination by foreign DNA molecules. Entire brain tissue was placed in sterile Petri dishes and was finely chopped with a scalpel in all directions until mashed. $1 \mathrm{ml}$ of PBS was added to the Petri dish, the suspension was mixed using a pipette 
and was passed thru a cone shaped mesh into a screw top Eppendorf tube. The Eppendorf containing the PBS/tissue solution was spun for two minutes at $13000 \mathrm{rmp}$ in a $5415-\mathrm{D}$ centrifuge. The resulting pellets were pinkish/yellow containing both tissue and blood. The supernatant was removed, the tube was spun briefly and any excess PBS was removed. $1 \mathrm{ml}$ of 1 xSSC was added to the pellet and the mixture was vortexed thoroughly in order to completely re-suspend the pellet. $10 \mu \mathrm{l}$ of a $10 \%$ SDS solution was added in order to lyse any remaining somatic cells. The tube was inverted several times to mix and then it was spun at $13000 \mathrm{rpm}$ for two minutes and a white pellet became visible.

The supernatant was then discarded, and $960 \mu$ of $0.2 \times$ SSC was added. The sample was vortexed thoroughly and $70 \mu \mathrm{l}$ of $\beta$-mercaptoethanol, $100 \mu \mathrm{l}$ of $10 \%$ and $20 \mu \mathrm{l}$ of $25 \mathrm{mg} / \mathrm{ml}$ Proteinase $\mathrm{K}$ were added. Tubes were then incubated at $37{ }^{\circ} \mathrm{C}$ for 2-3 hours and inverted occasionally. To clean up the DNA, two Phenol: Chloroform extractions and 1 chloroform extraction were performed in $15 \mathrm{ml}$ phase lock gel tubes 'Maxtract High Density' from Qiagen after the incubation. Ethanol precipitation was performed using $6 \mathrm{ml}$ of $100 \%$ ethanol and $300 \mu \mathrm{l}$ of $3 \mathrm{M}$ sodium acetate. The precipitated DNA was then carried in a new tube with $1 \mathrm{ml}$ of $80 \%$ ethanol. Following a quick spin, most of the ethanol was removed, and the tube was left in the fridge till the sample was needed.

\subsection{Sperm DNA extraction}

All DNA extractions were performed in a Category II flow hood (Walker Safety Cabinets Ltd, Glossop, UK) to minimize risk of contamination by 
foreign DNA molecules. Both caudal epidydimi from each animal were used for the DNA extraction.

Caudal epidydimi were placed in sterile Petri dishes and excess fat was cut off. Tissues were finely chopped in all directions. $1 \mathrm{ml}$ of PBS was added to the Petri dish, the suspension was mixed using a pipette and was passed threw a cone shaped mesh into a screw top Eppendorf tube. The tissue in the PBS was transferred from the Petri dish into the top of the cone. $5 \mu \mathrm{l}$ of the PBS/issue solution was placed onto a haemocytometer in order to visualize the ratio of sperm to somatic tissue. The Eppendorfs containing the PBS/tissue solution were then spun for two minutes at $13000 \mathrm{rmp}$ in a 5415D centrifuge. The resulting pellets were pinkish/yellow containing both tissue and blood. Supernatant was removed and spun briefly and any excess PBS was removed. $1 \mathrm{ml}$ of $1 \times \mathrm{SSC}$ was added to the pellet and the mixture was vortexed thoroughly in order to completely re-suspend the pellet. $10 \mu \mathrm{l}$ of a $10 \%$ SDS solution was added in order to lysis any remaining somatic cells. The tube was inverted several times to mix and then it was spun at 13000 rmp for two minutes and a white pellet became visible.

The supernatant was then discarded, and $960 \mu$ l of $0.2 \times$ SSC was added. The sample was vortexed thoroughly and $5 \mu \mathrm{l}$ of the solution was visualized in the microscope once again. No somatic cells should be visualized this time around. $70 \mu \mathrm{l}$ of $\beta$-mercaptoethanol, $100 \mu$ of $10 \%$ and $20 \mu \mathrm{l}$ of $25 \mathrm{mg} / \mathrm{ml}$ Protease $\mathrm{K}$ were added to the samples. Tubes were then incubated at $37^{\circ} \mathrm{C}$ for $2-3$ hours and inverted occasionally. To clean up the DNA, 2 Phenol: Chloroform extractions and 1 chloroform extraction were performed in phase lock gel tubes 'Maxtract High Density' (100x15 ml) from 
Qiagen after the incubation. Ethanol precipitation was performed using $6 \mathrm{ml}$ of $100 \%$ ethanol and $300 \mu \mathrm{l}$ of $3 \mathrm{M}$ sodium acetate. The precipitated DNA was then carried in a new tube with $1 \mathrm{ml}$ of $80 \%$ ethanol. Following a quick spin, most of the ethanol was removed, and the tube was left in the fridge till the sample was needed.

\subsubsection{DNA digestion and precipitation}

The $80 \%$ ethanol was removed and the pellet was left to air-dry for 30 minutes. The pellet was then re-suspended in $30-40 \mu \mathrm{l}$ of PCR-clean water. $15.5 \mu \mathrm{l}$ of the un-quantified sperm or brain genomic DNA was digested in a $20 \mu$ reaction containing $1 \times$ NEB buffer (New England Biolabs), $20 \mathrm{ng}$ of bovine serum albumin (BSA) and $25 \mathrm{U}$ of Msel enzyme, and incubated at $37^{\circ} \mathrm{C}$ in a water bath for 2 hours.

Then $60 \mu \mathrm{l}$ of $100 \%$ ethanol and $10 \% 3 \mathrm{M} \mathrm{NaAc}(\mathrm{pH} 5.2)$ was added to the DNA sample and the sample was left for a minimum of two hours at $80^{\circ} \mathrm{C}$. At the end of the two hours the sample was centrifuged in a bench-top Eppendorf 5415-D centrifuge at the maximum speed of $13,000 \mathrm{rpm}$ for 20 in each orientation. $100 \%$ ethanol was then substituted with $50 \mu \mathrm{l}$ of $80 \%$ ethanol. The sample was centrifuged again for five minutes and $80 \%$ ethanol was re-suspended in $5 \mathrm{mM}$ Tris.

\subsubsection{DNA quantification}

DNA concentrations were measured with a ND1000 spectrophotometer (NanoDrop Technologies). DNA concentration was 
calculated and samples were diluted to a concentration of $10 \mathrm{ng} / \mu \mathrm{l}$ using 5 $\mathrm{mM}$ Tris.

\subsubsection{Dilution of samples for Single-Molecule PCR optimization}

Before SM-PCR could be performed, the single-molecule concentration was established. The $10 \mathrm{ng} / \mu \mathrm{l}$ stock DNA was diluted using dilution buffer ( $5 \mathrm{mM}$ Tris $\mathrm{HCl} \mathrm{pH}$ of 7.5 and $5 \mu \mathrm{g} / \mathrm{ml}$ salmon sperm DNA) to the following concentrations $100 \mathrm{pg}, 50 \mathrm{pg}, 20 \mathrm{pg}, 10 \mathrm{pg}, 5 \mathrm{pg}$ and $2 \mathrm{pg}$, ready for PCR amplification of the ESTR locus Ms6-hm. Preliminary PCR reactions were performed on each specimen using the expand high fidelity PCR system from Roche (Mannheim, Germany) to optimize the DNA concentrations that yield the single-molecule level (concentration yielding $50 \%$ positive reactions). Eight replicates of each PCR assay at each concentration were set in 96-well PCR tube strips.

\subsubsection{Polymerase Chain Reaction}

A master-mix was prepared for the high fidelity PCR system with the components listed in the table below and the volume of the Master-mix as given was enough for the optimization of two different DNA samples. The PCR procedure used was based on that of Saiki and co-authors (Saiki et al., 1988). Master mix components and volumes are presented in Table 2.1. PCR buffer and enzyme mix used where obtained from the high fidelity PCR system kit. The kit contained a mix of Taq DNA polymerase and Tgo DNA polymerase. The 10x PCR buffer in the kit contained $15 \mathrm{mM} \mathrm{MgCl}_{2}$. 
Table 2.1. Master mix components.

Table shows the master mix components and volumes used for the S.M PCR technique.

\begin{tabular}{|l|c|c|}
\hline Components & 1 x reaction & Final concentration \\
\hline $10 \times$ PCR buffer & 1 & $1 \times$ PCR buffer \\
\hline dNTPs $(2 \mathrm{mM})$ & 1 & $200 \mu \mathrm{M}$ \\
\hline Forward primer $(10 \mathrm{mM})$ & 0.4 & $0.4 \mathrm{mM}$ \\
\hline Reverse primer $(10 \mathrm{mM})$ & 0.4 & $0.4 \mathrm{mM}$ \\
\hline Betaine $(4 \mathrm{M})$ & 2.5 & $1 \mathrm{M}$ \\
\hline Water & 3.4 & --- \\
\hline Enzyme mix $(3.5 \mathrm{U} / \mu \mathrm{l})$ & 0.3 & Variable \\
\hline DNA (required conc.) & 1 & \\
\hline
\end{tabular}

The primers used to amplify Ms6-hm where:

HM1.1F (5'GGGCAGGGCAGGGCAGGGCAGG3')

HM1.1R (5'CCCTGCCCTGCCCTGCCCTGCCC3').

Betaine was purchased from Sigma-Aldrich (Poole UK). All water used was ultra purified (Purite Select) and then UV irradiated (UVP Canada). $10 \mu \mathrm{l}$ PCR reactions were performed in $0.2 \mathrm{ml}$ strips on a PCT-225 DNA Engine Tetrad $^{\mathrm{TM}} 2$ Thermal Cycler (MJ Research, USA) and the PCR conditions used are presented in Table 2.2. 
Table 2.2. PCR conditions used for BALB/c inbred mice.

\begin{tabular}{|c|c|c|}
\hline Temperature & Time & Cycles \\
\hline $96^{\circ} \mathrm{C}$ & $3 \mathrm{mins}$ & $\times 1$ \\
\hline $96^{\circ} \mathrm{C}$ & $20 \mathrm{sec}$ & $\times 29$ \\
\hline $58^{\circ} \mathrm{C}$ & $30 \mathrm{sec}$ & \\
\hline $68^{\circ} \mathrm{C}$ & $3 \mathrm{mins}$ & $\times 1$ \\
\hline $68^{\circ} \mathrm{C}$ & $10 \mathrm{mins}$ & \\
\hline $15^{\circ} \mathrm{C}$ & For ever & \\
\hline
\end{tabular}

\subsubsection{Gel for single- molecule PCR optimization}

The gel used for single molecule PCR optimization was the $1 \%$ LEagarose gel containing $0.5 \times$ TBE (FMC Byproducts) with four sets of twentysix teeth combs. When the PCR process was completed, each tube had an addition of $5 \mu \mathrm{l}$ of $5 \times$ loading dye $(5 \times \mathrm{TAE}, 12.5 \%$ Ficoll $400,0.1 \%$ Bromophenol Blue) and $5 \mu \mathrm{l}$ of each of the PCR products was loaded in the $1 \%$ LE-agarose gel with $200 \mathrm{ng}$ of $1 \mathrm{~kb}$ DNA ladder. The samples where then set for electrophoresis at a voltage of $150-200 \mathrm{~V}$ for one or two hours or to 20 V overnight.

\subsubsection{Southern blotting}

The blotting procedure was based on that of Southern (Southern, 1975). At the end of the electrophoresis, the agarose gel was inverted on a tray and was rinsed while shaking on a platform twice for five minutes in a depurinating solution $(0.25 \mathrm{MHCl})$ as depurinated DNA is more readily cleaved by $\mathrm{NaOH}$. Then the gel was rinsed twice for ten minutes in 
denaturing solution $(0.5 \mathrm{M} \mathrm{NaOH}, 1 \mathrm{M} \mathrm{NaCl})$ and at the end, twice for five minutes in neutralizing solution $(0.5 \mathrm{M}$ Tris, $3 \mathrm{M} \mathrm{NaCl})$. Each time fresh blotting solution was used. The gel was then placed on a blotting apparatus containing $20 \times$ SSC and space around the gel was covered with Saran wrap. Nylon transfer membrane (MAGNA nylon-MSI, Osmonics) was soaked in $2 \mathrm{x}$ SSC and was placed directly on top of the gel followed by $43 \mathrm{MM}$ Whatman papers (pre-soaked in $2 \times \mathrm{SSC}$ ) all cut to the same size as the agarose gel. Precaution was taken that no air bubbles were trapped between the gel, nylon and papers. Finally, a stack of green paper towels, a glass sheet and weights were placed on top of the Whatman papers. The blotting apparatus was left overnight or for a minimum of two hours and DNA was transferred to the MAGNA nylon membrane via capillary transfer. The membrane was then placed in a $3 \mathrm{MM}$ folded paper and dried for 15 minutes in an $80^{\circ} \mathrm{C}$ oven. DNA was then covalently linked to the membrane by exposure to $7 \times 10^{*} 4$ $\mathrm{J} / \mathrm{cm}^{2} \mathrm{UV}$ in an Amersham UV cross-linker.

\subsubsection{HM1 probe construction}

Stocks of double-stranded probe DNA $5 \mathrm{ng} / \mu \mathrm{l}$, were generated by PCR amplification of the Ms6-hm ESTR locus by R. C. Barber. These probes were created by PCR amplification of synthetic primers containing the appropriated repeat unit in a $20 \mu \mathrm{l}$ reaction. The PCR reactions were performed using $2 \mu \mathrm{l} \mathrm{PCR}$ buffer, $1 \mu \mathrm{M}$ of forward primer (HM1.1F), $1 \mu \mathrm{M}$ reverse primer $(\mathrm{HM} 1.1 \mathrm{R})$ and $2.5 \mathrm{U} / \mu \mathrm{l}$ Taq polymerase (ABgene). No DNA input was necessary as the repeat specific primers acted as templates. Amplification was performed in 96 well plates (ABgene), with $20 \times\left[\left(96^{\circ} \mathrm{C}\right.\right.$ for 
$20 \mathrm{sec})\left(70^{\circ} \mathrm{C}\right.$ for $\left.\left.20 \mathrm{sec}\right)\right]$, then $40 \times\left[\left(96^{\circ} \mathrm{C}\right.\right.$ for $\left.30 \mathrm{sec}\right)\left(70^{\circ} \mathrm{C}\right.$ for $1 \mathrm{~min} 30$ sec)]. These probes were then purified using a QIAquick PCR Purification Kit (Qiagen).

\subsubsection{Radioactive labelling of probe and recovering}

Probes were radioactively labelled based on Random Prime incorporation (Feinberg \& Vogelstein, 1983; Feinberg \& Vogelstein, 1984), 4 $\mu \mathrm{l}$ of HM1 double stranded DNA probe $(5 \mathrm{ng} / \mu \mathrm{l})$ and $2 \mu \mathrm{l}$ of $1 \mathrm{~kb}$ ladder $5 \mathrm{ng}$ / $\mu \mathrm{l}$ (or $2 \mu \mathrm{l}$ of $200 \mathrm{bp}$ ladder $(10 \mathrm{ng} / \mu \mathrm{l}$ ) in the case of long gels) were added to $26 \mu \mathrm{l}$ of water and boiled for 6 minutes. The annealed probe was directly set on ice and $12 \mu \mathrm{g}$ of BSA (DNAse free at approximately $10 \mathrm{mg} / \mathrm{ml}$ ), $6 \mu \mathrm{l}$ of oligo labeling buffer (OLB) and $8.16 \mathrm{U}$ of Klenow enzyme (8,160 U / ml, USB) were added to the mixture. To this $1.5 \mu \mathrm{l}$ of ${ }^{32} \mathrm{P} \alpha-\mathrm{dCTP}$ was added in the radioactive area and the labeled probe was incubated for $2-5$ hours at $37^{\circ} \mathrm{C}$ or overnight at room temperature. The probe was recovered from unincorporated deoxyribonucleotides by ethanol precipitation using $100 \mu \mathrm{g}$ high molecular weight salmon sperm DNA (Sigma-Aldrich, Poole, UK) as a carrier. Probes were dissolved in $600 \mu \mathrm{l}$ of distilled water, and were boiled for 3 minutes prior to use.

\subsubsection{Hybridization}

A maximum of two membranes were placed into a hybridization bottle with church buffer ( $0.5 \mathrm{M} \mathrm{Na}$ Phosphate $7 \%$ SDS, $1 \mathrm{mM}$ EDTA) in a $65^{\circ} \mathrm{C}$ prewarmed hybridization oven at least 15 minutes prior to hybridization. The prehybridization solution was poured off the bottle and $10 \mathrm{ml}$ of church 
containing the probe were added. The bottle was left for a minimum of five hours or overnight at $65^{\circ} \mathrm{C}$ in the mini-10 hybridization oven (ThermoScientific, Ashford, UK).

\subsubsection{Washing Membranes}

Hybridization solution was poured off and $30 \mathrm{ml}$ of phosphate wash solution PWS (40 mM NaHPO 4 and $0.5 \%$ SDS) were added for 10 minutes. The wash solution was poured off and the number of counts was checked with a geiger before adding $50-100 \mathrm{ml}$ of high stringency wash solution HSW (0.1 $\times$ SSC and $0.01 \%$ SDS). The bottle was returned to the oven for 10 minutes and this procedure was repeated using fresh HSW several times, until the wash solution recovered after washing was less than 5 counts/second.

\subsubsection{Autoradiography}

Membranes were removed from the hybridization bottle and placed individually into an appropriately sized autoradiograph cassette (Genetic Research Instrumentation, Braintree, UK). An appropriately sized piece of Fuji Rx100 X-ray film (FijiFilm, USA) was placed directly onto the membrane. The cassette was sealed and put into the $-80^{\circ} \mathrm{C}$ freezer where it was left overnight or up to one week, depending on the strength of the signal.

\subsubsection{Developing films}

The films where developed manually using the appropriate developing solutions. 


\subsubsection{Evaluating single molecule concentration}

Following the step of single-molecule optimization (Section 2.2.5), southern blotting (Section 2.2.8), and DNA hybridization (Section 2.2.11), the concentration yielding $50 \%$ of positive PCRs was identified and recorded as the single-molecule concentration. If for example there was a case where one concentration had six positives and the next concentration had two-three positives then an average of the two concentrations was taken as the right single molecule concentration.

\subsubsection{Single molecule PCR}

The single molecule concentration found in Section 2.2.15 was then used to set up 96-well plate PCR reactions using the same PCR buffer composition and PCR conditions as described in Section 2.2.6 and the amplified products were run on agarose gel (Section 2.2.7), southern blotted (Section 2.2.8) and hybridized with radioactive probe as previously described. All wells containing a positive reaction were labelled at the corresponding position on the 96-well plate.

\subsubsection{Identification of positive PCR reactions}

The number of positive and negative reactions was counted and the probability of all negative reactions was calculated in order to approximately find the number of genome equivalent in each PCR reaction. All plate positions containing positive $\mathrm{PCR}$ reactions were marked and an average of 48 positives and 48 negatives was expected for the single molecule level. All positive reactions that contained a single allele for mutation scoring where used to run long gels. 


\subsubsection{Long gels}

Long gels were made by $6 \mathrm{~g}$ of LE Agarose (FMC Bioproducts) and $600 \mathrm{ml}$ of $1 \times$ TBE buffer containing Ethidium Bromide $5 \mathrm{mg} / \mathrm{ml}$. Casting trays $40 \mathrm{~cm}$ long and 27-tooth combs were used. The tanks was balanced and filled with $1 \times$ TBE. The 100 bp DNA stepladder (Promega marker, 200 $\mathrm{ng}$ ) and the $1 \mathrm{~kb}$ ladder (Invitrogen, $1 \mathrm{mg} / \mu \mathrm{l}$ stock) were loaded next to the DNA samples. The $1 \mathrm{~kb}$ ladder (Invitrogen) was used as a visible marker for tracking the distance migrated by the ladder DNA fragments. The wells where filled with $5 \mu$ l of each positive PCR reaction and the gel was left to run for up to 24 hours at $100-200$ Volts until the visible $2 \mathrm{~kb}$ ladder marker had reached the end of the gel. A UV wand (Chromato-vue UVM-57) from Ultra Violet Products Life Sciences (Cambridge, UK) was used to visualize the 1 $\mathrm{kb}$ ladder migration. The top half of the gel was cut and discarded, and the bottom half containing the ESTR PCR products retained for Southern blotting.

\subsubsection{Southern blotting long gels}

Same procedure was used as for short gels in Section 2.2.7.

\subsubsection{Hybridising of long gels}

Hybridization of long gels was performed as described in section 2.2.11, except here the 200 bp ladder was probed (Section 2.2.10). The nylon transfer membranes were hybridized with Ms6-hm radioactive probes and visualized by X-ray autoradiography as described in Section 2.2.13. 


\subsubsection{Pig-A assay}

\subsubsection{Blood Harvest}

Blood was harvested via heart puncture, using a 25 gauge needle $(0,5$ $x 16 \mathrm{~mm}$ ) and syringe. To prevent coagulation, the Eppendorf tubes in which the blood was kept contained heparinized beads. Approximately $200 \mu \mathrm{L}$ of blood was obtained per mouse. Blood was stored on ice or in a refrigerator until staining (same day). While $200 \mu \mathrm{L}$ were collected per mouse; only 30 $50 \mu \mathrm{L}$ are required to provide $2 \times 10^{8}$ cells for staining.

\subsubsection{Cell staining}

Each heparinized whole blood specimen was diluted 1:1 with PBS. One part diluted blood (typically $200 \mu \mathrm{L}$ ) was layered on top of eight parts room temperature Lympholyte Mammalian Cell Separation Reagent in a 15 $\mathrm{mL}$ conical polypropylene tube. To enrich for erythrocytes, specimens were centrifuged at $800 \times g$ for $20 \mathrm{~min}$. The resulting supernatants were carefully aspirated.1mL of PBS was gently added to the side of each tube (trying not to disturb the erythrocyte pellets). The PBS rinse solution was removed and a second addition of PBS (5 ml/tube) was added as before. Again, the supernatant was removed.

Each erythrocyte pellet was transferred to a clean $15 \mathrm{~mL}$ polypropylene tube containing $10 \mathrm{~mL}$ PBS, using a Pipetman. The samples were washed twice with $10 \mathrm{ml}$ PBS, the first time for $10 \mathrm{~min}$ and the second time for $5 \mathrm{~min}$ at $300 \mathrm{~g}$. Finally the samples were re-suspended in $5 \mathrm{~mL}$ of PBS and placed on ice. $2 \times 10^{8}$ cells were transferred to a tube containing $100 \mu \mathrm{L}$ antibody solution $(10 \mu \mathrm{L}$ stock anti-CD24-PE, $90 \mu \mathrm{L}$ PBS, $2 \% \mathrm{v} / \mathrm{v}$ 
foetal bovine serum). Cells were incubated for $30 \mathrm{~min}$ on ice and then centrifuged for 10 minutes. Supernatant was removed and pellet washed with10 mL PBS per tube.

Samples were finally centrifuged at $800 \times g$ for $10 \mathrm{~min}$ and the resulting supernatants were carefully aspirated. Pellets were re-suspended with $1 \mathrm{~mL}$ PBS. Samples were incubated for $30 \mathrm{~min}$ at room temperature, placed on ice and analysed within approximately 2 hours.

\subsubsection{Flow cytometric analysis}

Flow cytometric analysis was conducted using a FACSCanto ${ }^{\text {TM }}$ II flow cytometer and BD FacsDiva (version 6.1.3) software package.

\subsubsection{Data acquisition}

For analyses that required measuring the frequency of CD24-negative RBCs, data acquisition was triggered on the forward scatter parameter, and the sample rate was set to low. This facilitated efficient collection of $1 \times 10^{6}$ RBCs per specimen (typically $\leq 10 \mathrm{~min}$ ). The occurrence of the Pig-a mutation is recorded as the number of CD24-negative RBCs per one million RBCs. 


\section{Chapter 3. Results}

\subsection{Single Molecule PCR technique}

\subsubsection{Single Molecule -PCR optimisation}

Using a Single Molecule PCR (SM-PCR) technique, ESTR mutation frequencies were established in DNA samples extracted from sperm and brain. This technique involves the dilution of stock $10 \mathrm{ng} / \mu \mathrm{l}$ genomic DNA and amplification of multiple DNA samples, each containing approximately one amplifiable ESTR molecule. Single molecule concentration is determined as the concentration that yields approximately half positive and half negative reactions (Figure 3.1.1).

Figure 3.1.1. A representative image of an autoradiograph used for SMPCR optimisation.

An X-ray autoradiograph of a Southern blot containing eight replicate PCR products of Ms6-hm locus, amplified from a serial dilution of DNA samples. DNA was diluted to the concentrations ranging from 100 to $2 \mathrm{pg}$. In this experiment, the sample with the concentration of $5 \mathrm{pg}$ input DNA showed 4 positive reactions (50\%) and this concentration was later used for the SMPCR experiments.

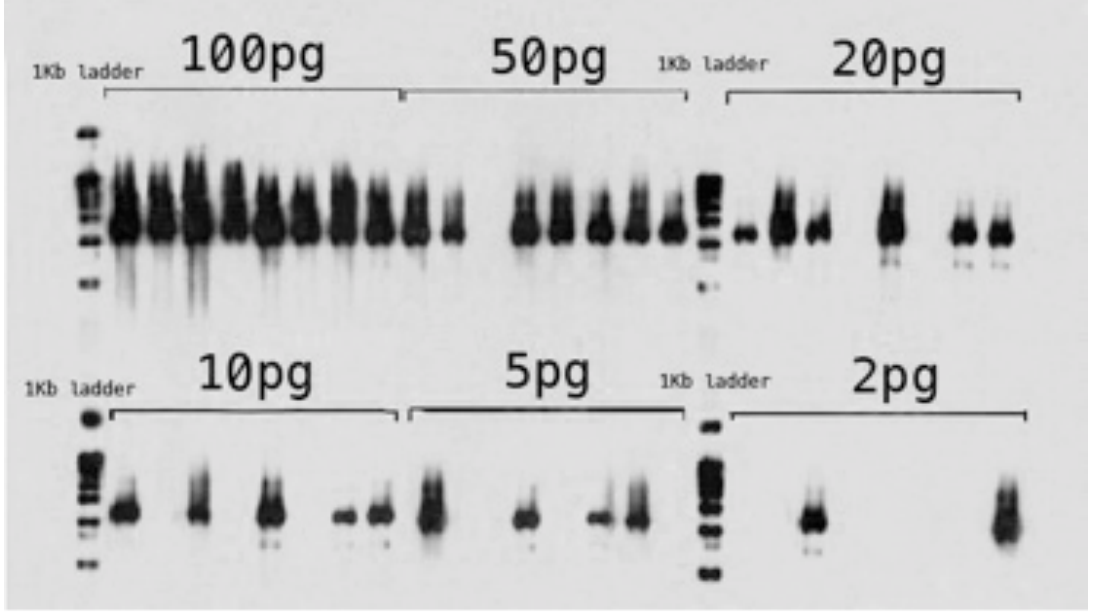




\subsubsection{Poisson analysis}

After the PCR optimisations, the Poisson analysis was performed. $1 \mu$ of DNA samples were diluted down to SM concentration in ninety-six reactions that were then amplified and checked for the presence of PCR products on an agarose gel.

For each DNA samples, a number of PCR reactions were run to produce approximately $90-120$ positive reactions. The number of negative PCR reactions was used to estimate the number of amplifiable Ms6-hm molecules (progenitors) in each sample (Figure 3.1.2).

Figure 3.1.2. A representative image of an autoradiograph used for poisson analysis.

An autoradiograph of a Southern blot containing 96 SM-PCR products hybridised with the Ms6-hm probe is shown. The SM-PCR products were detected in 52 reactions (positives), and 44 reactions yielded no products (negatives). Black arrows indicate positive reactions. White arrows indicate the lanes containing $1 \mathrm{~Kb}$ DNA ladder. 


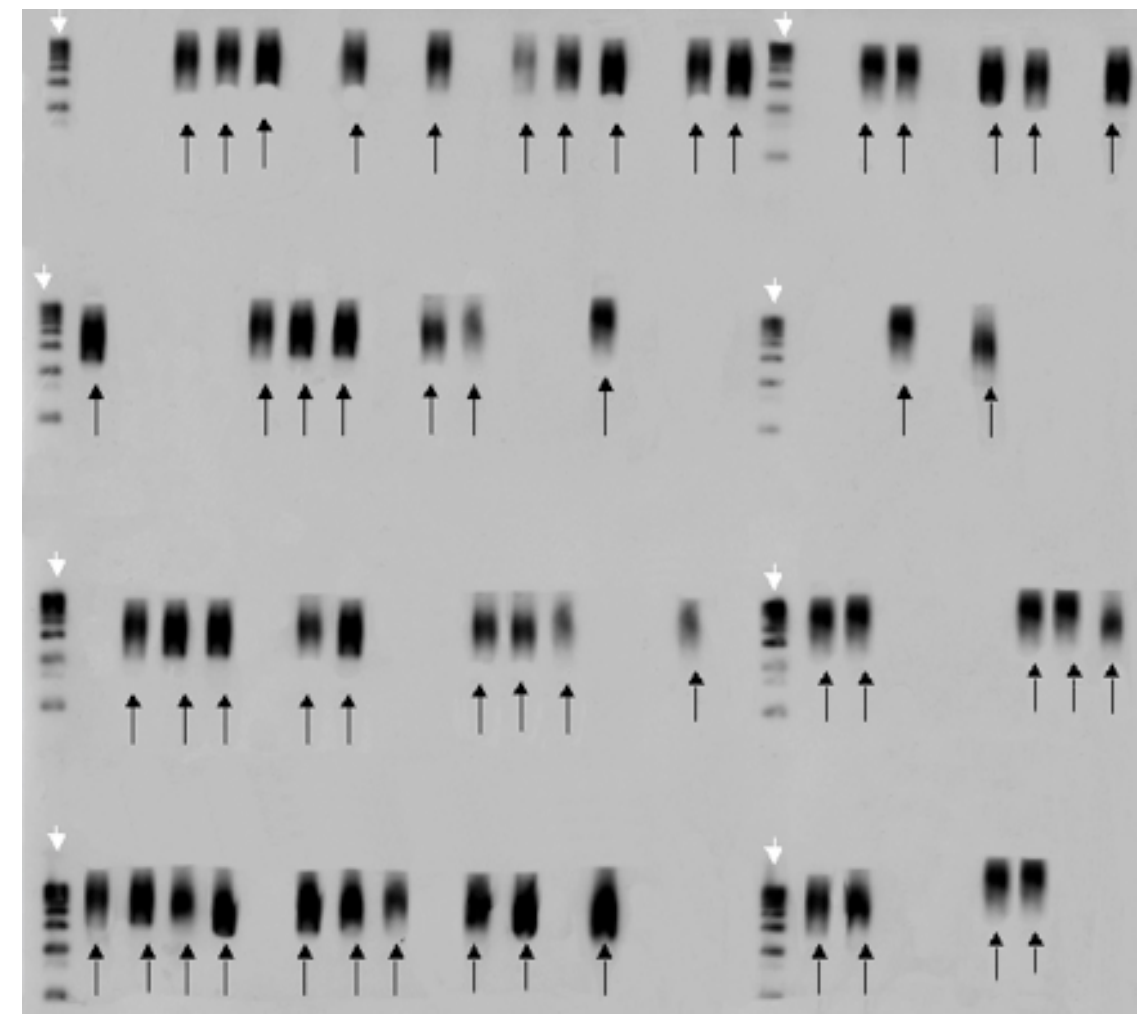

The Poisson distribution was used to determine the number of amplifiable molecules per reaction: $P^{-}(k=0)=\mathrm{e}^{\lambda}$ or $\lambda=-\ln \left(P^{-}\right)$

Were:

$K=$ total number of reactions

$\mathrm{n}^{-} \quad=$ number of negative reactions

$P^{-} \quad=$ the frequency of negative reactions ----> $\mathrm{P}^{-}=\mathrm{n}^{-} / \mathrm{K}$

$\lambda \quad=$ the number of amplifiable $M s 6-h m$ molecules per reaction.

\subsubsection{Mutation Scoring}

All positive samples were further analysed for the presence of ESTR mutations, which were resolved on $40-\mathrm{cm}$ long agarose gels. The size of Ms6-hm allele is approximately $2.5 \mathrm{~kb}$ with 400 repeats, each repeat being $5 \mathrm{bp}$ long. As in a number of previous studies on mutation detection at ESTR 
loci (Yauk et al., 2002; Dubrova 2005a; Barber et al., 2006; Barber et al., 2009; Glen et al., 2008), PCR products showing a shift of at least $1 \mathrm{~mm}$ relative to the progenitor allele were scored as mutant Ms6-hm molecules (Figure 3.1.3).

Figure 3.1.3. ESTR mutation detection at the Ms6-hm locus.

An autoradiograph of SM-PCR products resolved on a 40-cm log agarose gel and hybridised with the Ms6-hm probe is shown. DNA bands showing at least $1 \mathrm{~mm}$ difference from the progenitor alleles were recorded as mutants and are indicated by arrows. This distance of $1 \mathrm{~mm}$ is equivalent to approximately 2 Ms6-hm repeat units for the BALB/c alleles. Samples were run alongside 200bp DNA ladders used for their size.

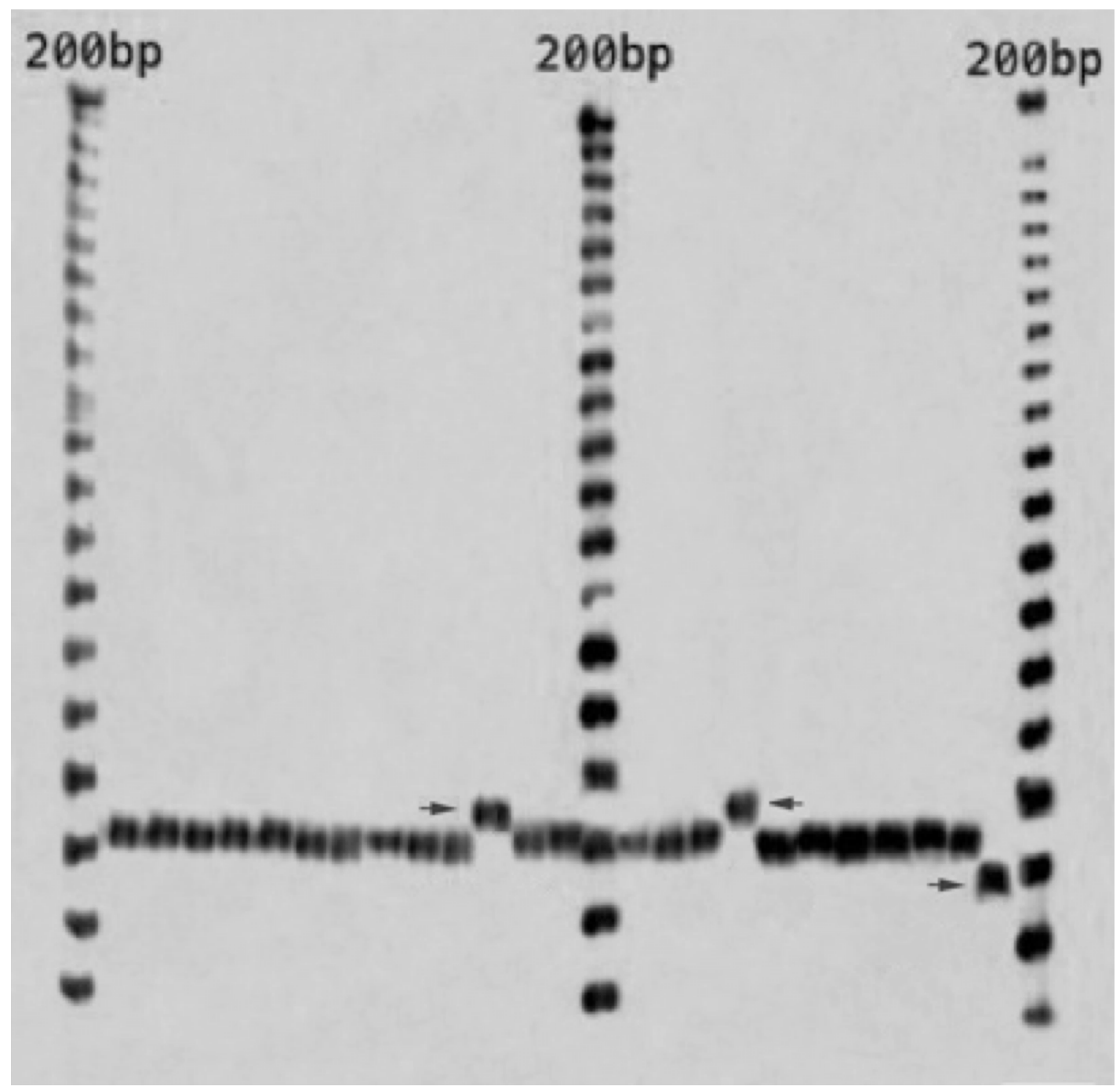




\subsubsection{Mutation mosaicism}

As previously reported (Barber et al., 2009), in some control and treated $\mathrm{BALB} / \mathrm{c}$ animals, as well as in their offspring, the presence of germline and somatic mosaicism was observed, where a similar mutation was detected in multiple DNA samples (Figure 3.1.4). Mosaicism at the Ms6-hm locus most likely results from the propagation of a single mutational event occurring during the first few cell divisions following fertilization (Kelly et al., 1989). As in previous ESTR studies (Dubrova et al., 1998; Barber et al., 2009), all cases of mosaicism were considered as 'a single independent mutation event' and the total number of unique mutations was used to estimate ESTR mutation frequency. In this mutation analysis, mosaic mutations were strictly considered to be those having five or more identical sized alleles in a given sample.

\section{Figure 3.1.4. Examples of singleton and mosaic ESTR mutations.}

Two autoradiographs of SM-PCR blots probed by the Ms6-hm probe are shown. The image on the left contains a singleton ESTR mutation; the image on the right represents two identical mosaic mutations. Mutants are indicated with arrows.

Singleton mutations

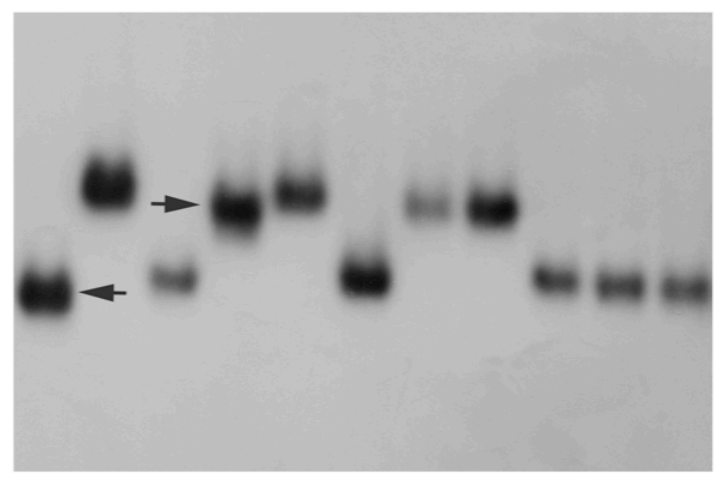

Mosaic Mutations

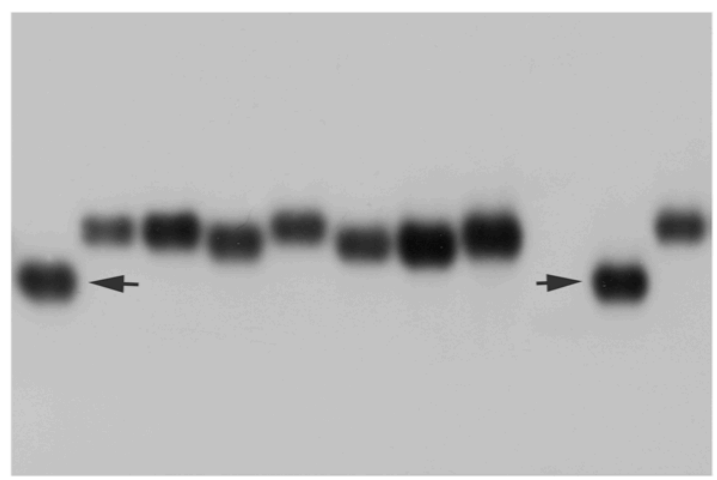




\subsubsection{ESTR mutation frequencies in control groups}

The frequency of ESTR mutation was firstly established in control male mice maintained on the diet containing normal levels of choline and folic acid and their first-generation offspring. Table 3.1.1 presents a summary of ESTR mutation data for all control males.

Table 3.1.1. ESTR mutation frequencies in controls (sperm).

\begin{tabular}{|l|c|c|c|}
\hline Group & No mutations & No progenitors & Frequency \pm s.e. \\
\hline$F_{0}$ Control A & 20 & 618 & $0.0324 \pm 0.0074$ \\
\hline$F_{0}$ Control B & 16 & 533 & $0.0300 \pm 0.0077$ \\
\hline$F_{0}$ Control C & 27 & 639 & $0.0423 \pm 0.0084$ \\
\hline$F_{1}$ Control B & 19 & 533 & $0.0356 \pm 0.0084$ \\
\hline$F_{1}$ Control C & 25 & 567 & $0.0441 \pm 0.0091$ \\
\hline All controls & 107 & 2890 & $0.0370 \pm 0.0037$ \\
\hline
\end{tabular}

Table shows the number of unique mutations detected at the locus Ms6-hm in DNA samples extracted from sperm tissue taken from $F_{0}$ and $F_{1}$ control BALB/c male mice, 8, 12, 18 and 22-weeks-old. The control data from all 8, 12,18 and 22 weeks were combined.

The frequency of ESTR mutation, $\mu$ in each tissue was estimated by dividing the number of mutants, $m$ by the total number of amplifiable DNA molecules, $\lambda$. The standard error of mutation frequency, se $\mu$ was estimated as:

$$
s e \mu=\mu \sqrt{\left(\frac{s e \lambda}{\lambda}\right)^{2}+\frac{1}{m}}
$$

where $\operatorname{se} \lambda$ is the standard error of the number of amplifiable DNA molecules. 
Table 3.1.2 presents a comparison of ESTR mutation frequencies in control males. As the frequencies did not significantly differ, the control data were combined.

Table 3.1.2. Statists for the difference between all control groups.

\begin{tabular}{|c|c|c|c|c|c|}
\hline & $\mathrm{F}_{0}$ Control $\mathrm{A}$ & $\mathrm{F}_{0}$ Control B & $\mathrm{F}_{0}$ Control $\mathrm{C}$ & $\mathrm{F}_{1}$ Control B & $\mathrm{F}_{1}$ Control $\mathrm{C}$ \\
\hline $\mathrm{F}_{0}$ Control $\mathrm{A}$ & - & $t=0.22$ & $t=0.88$ & $t=0.29$ & $t=1.00$ \\
\hline $\mathrm{F}_{0}$ Control B & $P=0.8222$ & - & $t=1.08$ & $t=0.49$ & $t=1.18$ \\
\hline $\mathrm{F}_{0}$ Control C & $P=0.3766$ & $P=0.2806$ & - & $t=0.56$ & $t=0.14$ \\
\hline $\mathrm{F}_{1}$ Control B & $P=0.7750$ & $P=0.6232$ & $P=0.5729$ & - & $t=0.69$ \\
\hline $\mathrm{F}_{1}$ Control C & $P=0.3187$ & $P=0.2371$ & $P=0.8845$ & $P=0.4926$ & - \\
\hline
\end{tabular}

Table shows the Student's test $(t)$ and probability for difference $(P)$ between the frequencies of ESTR mutation at the Ms6-hm locus in DNA samples extracted from sperm tissue taken from $F_{0}$ and $F_{1}$ control BALB/c male mice, 8, 12, 18 and 22-weeks-old. 
The frequencies of ESTR mutation in DNA samples extracted from the brain tissue of first-generation offspring of male mice maintained on a diet containing normal levels of choline and folic acid are given in Table 3.1.3. As ESTR mutation frequencies in two groups did not significantly differ, the control data were aggregated.

Table 3.1.3. ESTR mutation frequencies in controls (brain).

\begin{tabular}{|l|l|l|l|l|l|}
\hline & $\begin{array}{l}\text { No } \\
\text { mutations }\end{array}$ & $\begin{array}{l}\text { No } \\
\text { progenitors }\end{array}$ & Frequency \pm s.e. & $t^{*}$ & Prob \\
\hline F1 Control B & 17 & 499 & $0.0341 \pm 0.0085$ & - & - \\
\hline F1 Control C & 19 & 473 & $0.0402 \pm 0.0095$ & 0.48 & 0.6305 \\
\hline $\begin{array}{l}\text { Total } \\
\text { Control }\end{array}$ & 36 & 972 & $0.0370 \pm 0.0063$ & - & - \\
\hline
\end{tabular}

* Student's $t$ - test and probability for difference between the two control groups.

Table shows the frequency of unique ESTR mutations detected at the Ms6-hm locus in DNA samples extracted from the brain tissue of 8-week-old BALB/c male mice. The values of Student's $t$-test and probabilities ( $P$-values) are also shown. 
Figure 3.1.5 summarises all findings for ESTR mutation frequencies in DNA samples extracted from sperm and brain of all control males. Given that for each tissue the frequencies of ESTR mutation did not significantly differ between all control groups, sperm control data were aggregated, as were those for brain.

Figure 3.1.5. ESTR mutation frequencies in control mice.

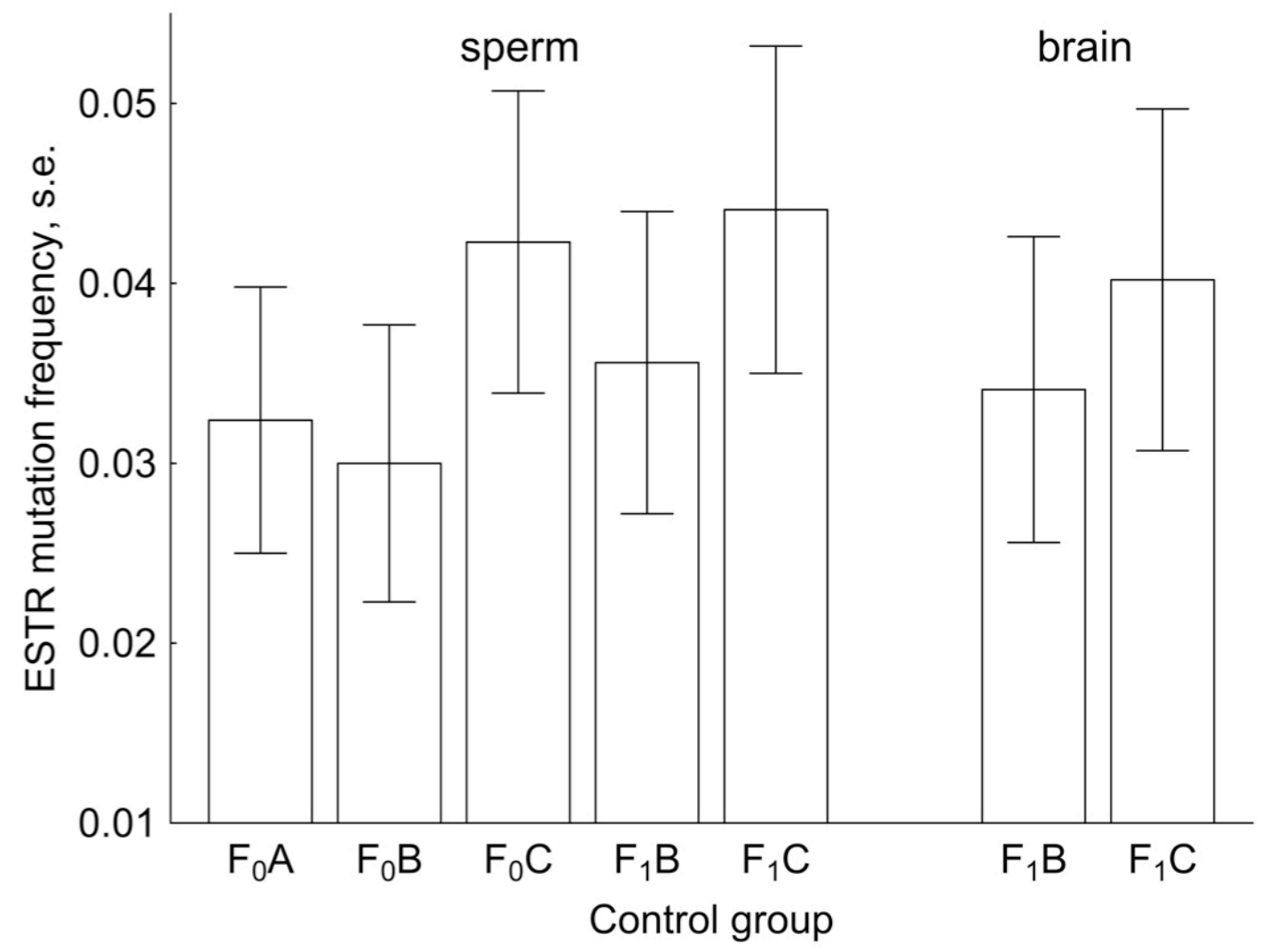

Graph represents the frequency of unique ESTR mutations detected at the Ms6-hm locus in DNA samples extracted from sperm and brain of $F_{0}$ and $F_{1}$ control male mice of $8,12,18$ and 22 weeks of age. Standard errors are shown. 
3.1.6. Effects of dietary methyl deficient diet on ESTR mutation induction

The frequency of ESTR mutation was evaluated in three groups of treated males sacrificed immediately after treatment $\left(F_{0}\right.$ Test $\left.A\right)$, or $6\left(F_{0}\right.$ Test B) and 10 weeks $\left(F_{0}\right.$ Test $\left.C\right)$ after the end of dietary restriction. Table 3.1.4 presents a summary of these data.

The table shows the frequency of unique mutations at the ESTR locus Ms6-hm in DNA samples extracted from sperm tissue taken from control and treated BALB/c male mice 12, 18 and 22 weeks of age. The values of Student's $t$-test and probabilities ( $P$-values) are also shown. 
Table 3.1.4. ESTR mutation frequencies in treated and control males (sperm).

\begin{tabular}{|c|c|c|c|c|c|c|}
\hline Group & $\begin{array}{l}\text { No } \\
\text { mutations }\end{array}$ & $\begin{array}{l}\text { No } \\
\text { progenitors }\end{array}$ & $\begin{array}{l}\text { Frequency } \\
\pm \text { s.e. }\end{array}$ & $\begin{array}{l}\text { Ratio } \\
\text { to } \\
\text { control }\end{array}$ & $t$ & Prob \\
\hline \multicolumn{7}{|l|}{ Control } \\
\hline $\begin{array}{l}\text { Group } \\
\text { A }\end{array}$ & 20 & 618 & $\begin{array}{l}0.0324 \pm \\
0.0074\end{array}$ & - & - & - \\
\hline $\begin{array}{l}\text { Group } \\
\text { B }\end{array}$ & 16 & 533 & $\begin{array}{l}0.0300 \pm \\
0.0077\end{array}$ & - & - & - \\
\hline $\begin{array}{l}\text { Group } \\
\text { C }\end{array}$ & 27 & 639 & $\begin{array}{l}0.0423 \pm \\
0.0084\end{array}$ & - & - & - \\
\hline $\begin{array}{l}\text { All } \\
\text { controls }\end{array}$ & 107 & 2890 & $\begin{array}{l}0.0370 \pm \\
0.0037\end{array}$ & - & - & - \\
\hline \multicolumn{7}{|l|}{ Test } \\
\hline \multirow[t]{2}{*}{$\begin{array}{l}\text { Group } \\
\text { A }\end{array}$} & 21 & 446 & $\begin{array}{l}0.0471 \pm \\
0.0106\end{array}$ & 1.27 & $0.90^{\circ}$ & $0.3702^{*}$ \\
\hline & & & & 1.45 & $1.14^{\dagger}$ & $0.2556^{\dagger}$ \\
\hline \multirow[t]{2}{*}{$\begin{array}{l}\text { Group } \\
\text { B }\end{array}$} & 20 & 622 & $\begin{array}{l}0.0302 \pm \\
0.0069\end{array}$ & 0.82 & $0.83^{n}$ & $0.4085^{\circ}$ \\
\hline & & & & 1.01 & $0.01^{\dagger}$ & $0.9851^{\dagger}$ \\
\hline \multirow[t]{2}{*}{$\begin{array}{l}\text { Group } \\
\text { C }\end{array}$} & 26 & 498 & $\begin{array}{l}0.0522 \pm \\
0.0106\end{array}$ & 1.41 & $1.36^{*}$ & $0.1749^{*}$ \\
\hline & & & & 1.24 & $0.73^{\dagger}$ & $0.4610^{\dagger}$ \\
\hline All Test & 67 & 1606 & $\begin{array}{l}0.0417 \pm \\
0.0052\end{array}$ & 1.13 & $0.73^{*}$ & $0.4638^{*}$ \\
\hline
\end{tabular}

"Student's test and probability for difference from mutation frequency in the total control group.

${ }^{\dagger}$ Student's test and probability for difference from mutation frequency in the age-matched control group. 
For each group of exposed males the frequency of ESTR mutation was compared with those in the combined control group and in the age-matched animals. An elevated frequency of ESTR mutation was detected in the group of males sacrificed immediately after treatment (Group A). However when compared with the corresponding frequencies in controls, this difference was not statistically significant. The frequency of ESTR mutation in DNA samples extracted from sperm of mice sampled 6 (Group B) and 10 (Group C) weeks after the end of dietary restriction was practically indistinguishable from those in the combined control group and in the age-matched animals. Figure 3.1.6 summarizes the data obtained by profiling the exposed males and agematched control groups.

\section{Figure 3.1.6. ESTR mutation frequencies in sperm.}

Graph represents a comparison of ESTR mutation frequencies in sperm of age-matched control (white bars) and (grey bars) treated male mice. The three groups of treated and control $\mathrm{F}_{0}$ male mice are plotted; standard errors are shown.

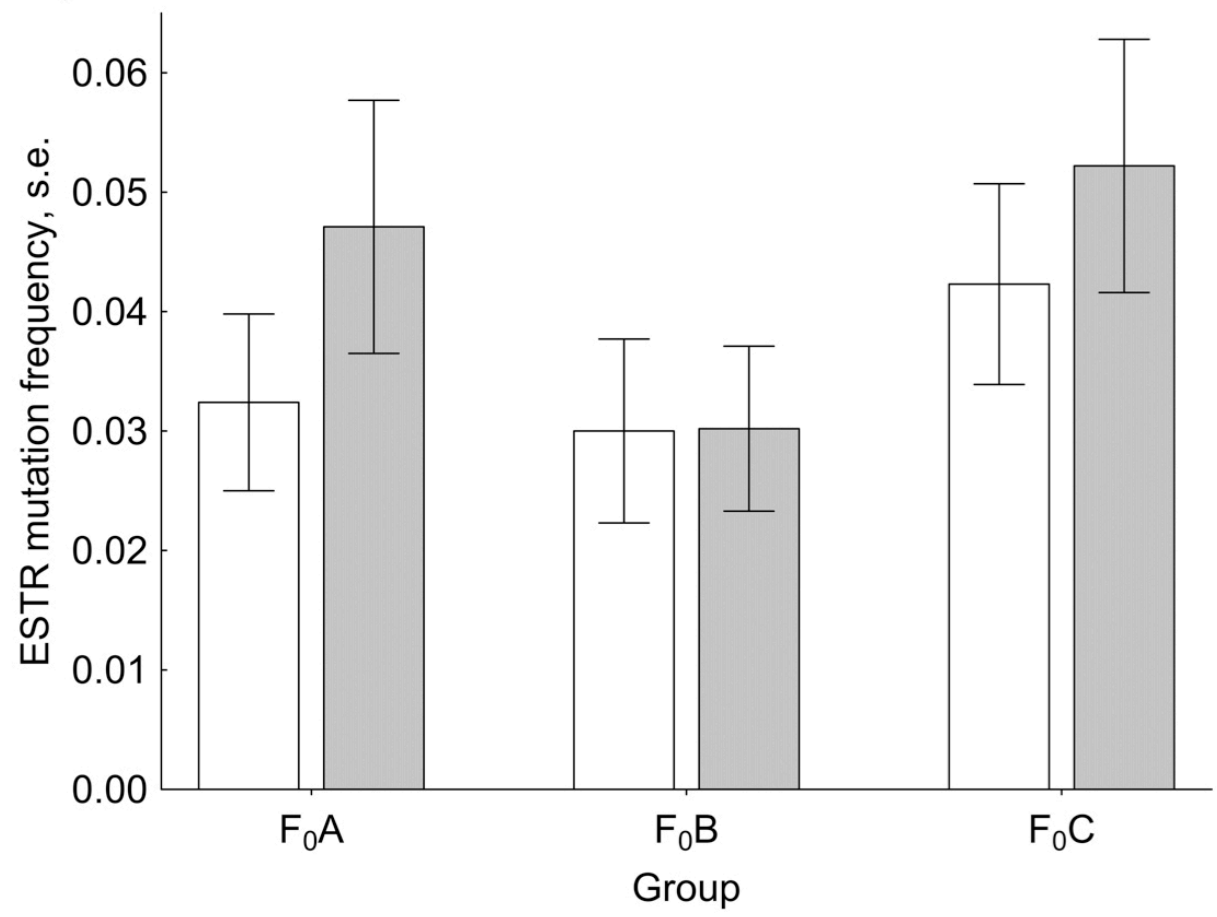


In conclusion, the results presented in this chapter suggest that 8weeks of low dietary methyl-donor intake does not affect ESTR mutation rate in the germline of treated male mice sampled either immediately after treatment, or 6 and 10 weeks after the end of dietary restriction.

\subsubsection{Transgenerational effects of paternal methyl-donor deficiency}

To establish whether paternal dietary restriction can result in transgenerational changes, ESTR mutation frequencies were evaluated in DNA samples extracted from sperm and brain of the male offspring of treated males conceived approximately 2 and 6 weeks after the end of dietary restriction

Given that this study was designed to analyze the transgenerational effects of paternal diet, all the offspring of control and treated males were kept on a normal methyl-donor sufficient diet. As already mentioned, the frequency of ESTR mutation was established in the two groups of the $F_{1}$ offspring of treated males (Test $F_{1} B$ and Test $F_{1} C$ ), as well as in the offspring fathered by control males (Control $F_{1} B$ and Control $F_{1} C$, see Figure 3.1). Tables 3.1.5 and 3.1.6 summarize the data on ESTR mutation frequencies in sperm and brain of the offspring of control and treated males. 
Table 3.1.5. ESTR mutation frequencies in the offspring of treated and control males (sperm).

\begin{tabular}{|l|l|l|l|l|l|l|}
\hline Group & $\begin{array}{l}\text { No } \\
\text { mutations }\end{array}$ & $\begin{array}{l}\text { No } \\
\text { progenitors }\end{array}$ & $\begin{array}{l}\text { Frequency } \\
\pm \text { s.e. }\end{array}$ & $\begin{array}{l}\text { Ratio } \\
\text { to } \\
\text { control }\end{array}$ & $t$ & Prob \\
\hline $\begin{array}{l}\text { F }_{1} \\
\text { control }\end{array}$ & & & & & & \\
\hline Group B & 19 & 533 & $\begin{array}{l}0.0356 \pm \\
0.0084\end{array}$ & - & - & - \\
\hline Group C & 25 & 567 & $\begin{array}{l}0.0441 \pm \\
0.0091\end{array}$ & - & - & - \\
\hline $\begin{array}{l}\text { All } \\
\text { controls }\end{array}$ & 41 & 1100 & $0.0370 \pm$ & - & - & - \\
\hline F1 Test & & 0.0037 & & & \\
\hline Group B & 22 & 500 & $0.0440 \pm$ & 1.19 & $0.67^{*}$ & $0.5003^{*}$ \\
\hline Group C & 29 & 696 & $0.0417 \pm$ & 1.13 & $0.53^{*}$ & $0.5984^{*}$ \\
\hline All Diet & 51 & 1196 & $0.0426 \pm$ & 1.15 & $0.78^{*}$ & $0.4343^{*}$ \\
& & & 0.0062 & & & \\
\hline
\end{tabular}

"Student's test and probability for difference from mutation frequency in the total control group.

${ }^{\dagger}$ Student's test and probability for difference from mutation frequency in the age-matched control group.

Table presents the frequency of unique mutations at the ESTR locus Ms6-hm in DNA samples extracted from sperm tissue taken from the first-generation offspring of treated and control males 8-weeks of age. The values of Student's $t$-test and probabilities ( $P$-values) are also shown. 
Table 3.1.6. ESTR mutation frequencies in the offspring of treated and control males (brain).

\begin{tabular}{|c|c|c|c|c|c|c|}
\hline Group & $\begin{array}{l}\text { No } \\
\text { mutations }\end{array}$ & $\begin{array}{l}\text { No } \\
\text { progenitors }\end{array}$ & $\begin{array}{l}\text { Frequency } \\
\pm \text { s.e. }\end{array}$ & $\begin{array}{l}\text { Ratio to } \\
\text { control }\end{array}$ & $t$ & Prob \\
\hline \multicolumn{7}{|l|}{$\begin{array}{l}F_{1} \\
\text { control }\end{array}$} \\
\hline Group B & 17 & 499 & $\begin{array}{l}0.0341 \pm \\
0.0085\end{array}$ & - & - & - \\
\hline Group C & 19 & 473 & $\begin{array}{l}0.0402 \pm \\
0.0095\end{array}$ & - & - & - \\
\hline $\begin{array}{l}\text { All } \\
\text { controls }\end{array}$ & 36 & 972 & $\begin{array}{l}0.0370 \pm \\
0.0063\end{array}$ & - & - & - \\
\hline \multicolumn{7}{|l|}{$F_{1}$ Test } \\
\hline \multirow[t]{2}{*}{ Group B } & 17 & 487 & $\begin{array}{l}0.0349 \pm \\
0.0087\end{array}$ & 0.94 & $0.20^{*}$ & $0.8428^{*}$ \\
\hline & & & & 1.02 & $0.07^{\dagger}$ & $0.9448^{\dagger}$ \\
\hline \multirow[t]{2}{*}{ Group C } & 16 & 481 & $\begin{array}{l}0.0333 \pm \\
0.0085\end{array}$ & 0.90 & $0.36^{*}$ & $0.7217^{*}$ \\
\hline & & & & 0.83 & $0.54^{\dagger}$ & $0.5871^{\dagger}$ \\
\hline All Test & 33 & 968 & $\begin{array}{l}0.0341 \pm \\
0.0061\end{array}$ & 0.92 & $0.34^{*}$ & $0.7368^{\circ}$ \\
\hline
\end{tabular}

"Student's test and probability for difference from mutation frequency in the total control group.

${ }^{\dagger}$ Student's test and probability for difference from mutation frequency in the age-matched control group.

Table presents the frequency of unique ESTR mutations detected at the Ms6-hm locus in DNA samples extracted from brain tissue of the 8-week-old $F_{1}$ offspring of control and treated male mice. The values of Student's $t$-test and probabilities ( $P$-values) are also shown. 
Similarly to the approach used in the previous section, the frequency of ESTR mutation in the both $F_{1}$ groups fathered by exposed males were compared with those in the combined control group, as well as with the $F_{1}$ offspring of age-matched control males. As far as ESTR mutation frequency in the $F_{1}$ male germline is concerned, it was very close to that in controls. The same was also true for the brain data. Figure 3.1.7 summarizes the transgenerational data. In conclusion, the results presented in this chapter imply that paternal methyl-donor dietary restriction does not affect the ESTR mutation rates in their offspring.

Figure 3.1.7. ESTR mutation frequencies in the $F_{1}$ offspring of treated and control male mice.

Graph represents the frequencies of ESTR mutation in sperm and brain of 8week-old male $F_{1}$ offspring of control (white bars) and test (grey bars) male mice. Standard errors of ESTR mutation frequency are shown.

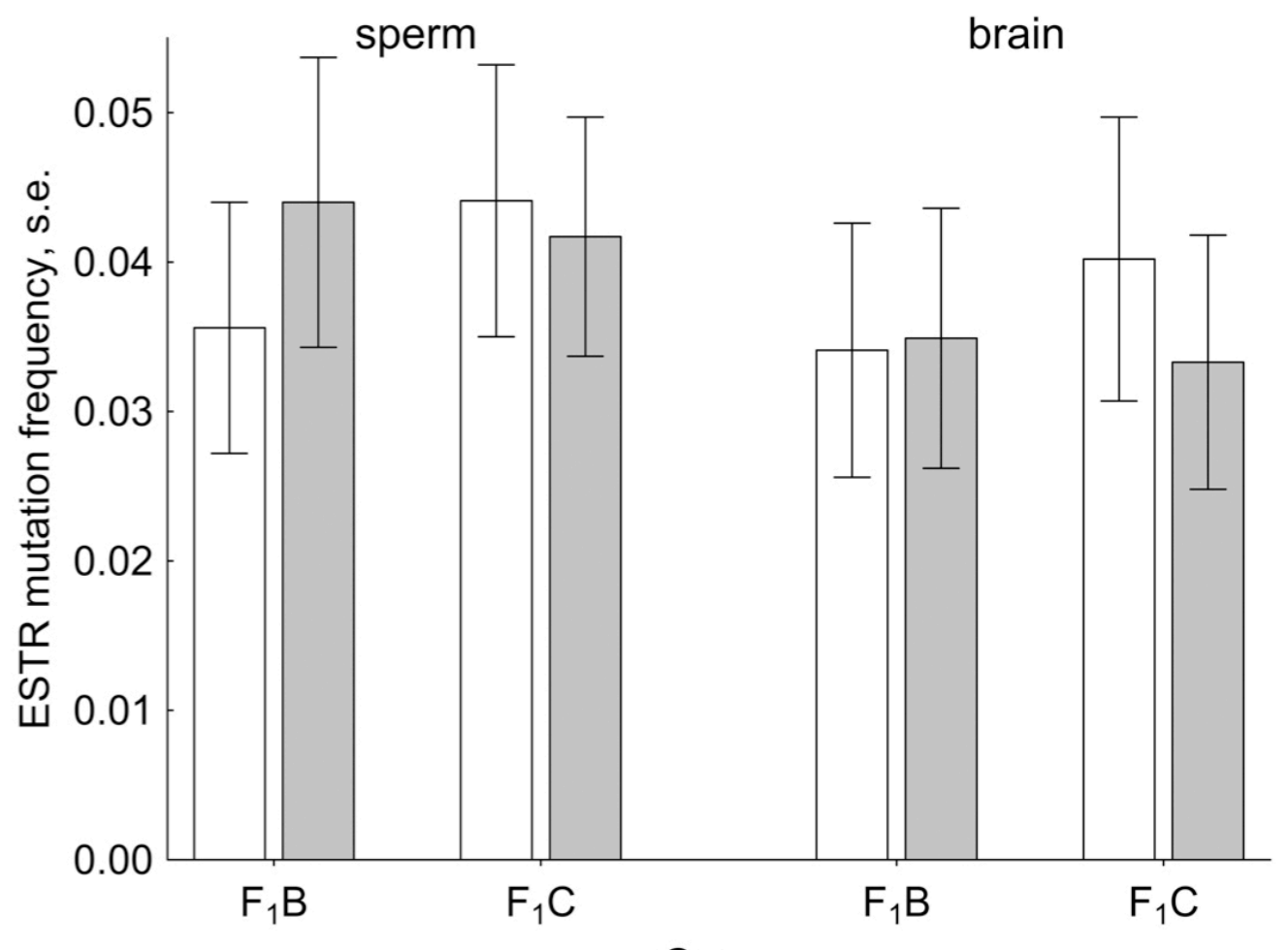

Group 


\subsubsection{Effects of a methyl-donor deficient diet on ESTR mutation} spectrum

The spectra of sperm mutations at the Ms6-hm locus were evaluated in all control and test groups of the $F_{0}$ and $F_{1}$ generation. The ESTR length changes were defined for the 294 unique mutations found in sperm of all control and treated males, as well as in sperm and brain tissues of their offspring. Following the approach adopted in the previous studies (Dubrova, 2005; Barber et al., 2009), the progenitor allele was assumed to be the nonmutant allele closest in size to the mutant allele (Figure 3.1.5).

The spectra of ESTR mutation were first established in the $F_{0}$ control groups. As the distribution of mutants did not significantly differ in the sperm tissue of control and test males, the data were combined. The incidence of gains and losses for DNA samples extracted from sperm of all $F_{0}$ males did not show significant bias toward gains or losses (Table 3.1.7). 
Table 3.1.7. ESTR mutation spectra in control and treated $F_{0}$ males (sperm).

\begin{tabular}{|l|l|l|l|}
\hline Group & Gains (\%) & Loses $(\%)$ & Total \\
\hline Control A & 11 & 9 & \\
\hline Control B & 6 & 10 & 20 \\
\hline Control C & 10 & 17 & 16 \\
\hline Control A+B+C & $27(42.86 \%)$ & $36(57.14 \%)$ & 63 \\
\hline & & & 27 \\
\hline Test A & 9 & 12 & 21 \\
\hline Test B & 14 & 6 & 20 \\
\hline Test C & 9 & 17 & 26 \\
\hline Test A+B+C & $32(47.76 \%)$ & $35(52.24 \%)$ & 67 \\
\hline$X^{2}, \mathrm{df}=1^{*}$ & 0.32 & $P=0.5746$ & \\
\hline Total & $59(45.38 \%)$ & $71(54.62 \%)$ & 130 \\
\hline$X^{2}, \mathrm{df}=1^{\dagger}$ & 1.11 & $P=0.2926$ & \\
\hline
\end{tabular}

* Chi-square test for difference between groups

${ }^{\dagger}$ Chi-square test for homogeneity of total number of gains of losses

Table shows the incidence of unique mutations involving gain or loss of repeat units at the Ms6-hm locus detected in DNA samples extracted from sperm of control and treated males of 12, 18 and 22 weeks of age. The values of chi-square test for the differences between groups and homogeneity within group and corresponding probabilities are also shown. 
The spectrum of ESTR mutations in DNA samples extracted from sperm of $F_{0}$ males is shown on Figure 3.1.8.

Figure 3.1.8. Spectra of ESTR mutations detected in DNA samples extracted from sperm of $F_{0}$ males.

Graph shows the combined data for ages 12, 18 and 22 weeks; all mosaic mutants were treated as a single mutational event. The number of repeats lost or gained are binned as $\pm 2-3$ repeats $( \pm 1), \pm 4-5$ repeats $( \pm 1), \pm 6-7$ repeats $( \pm 1), \pm 8-9$ repeats $( \pm 1), \pm 10-19$ repeats $( \pm 9)$, and $\pm 20+$ repeats $( \pm 20)$. White bars represent control groups, while grey bars represent their age matched test groups. The frequency distributions of the two groups do not significantly differ (Kolomogorov-Smirnov two-sample test, $P=0.9465$ ).

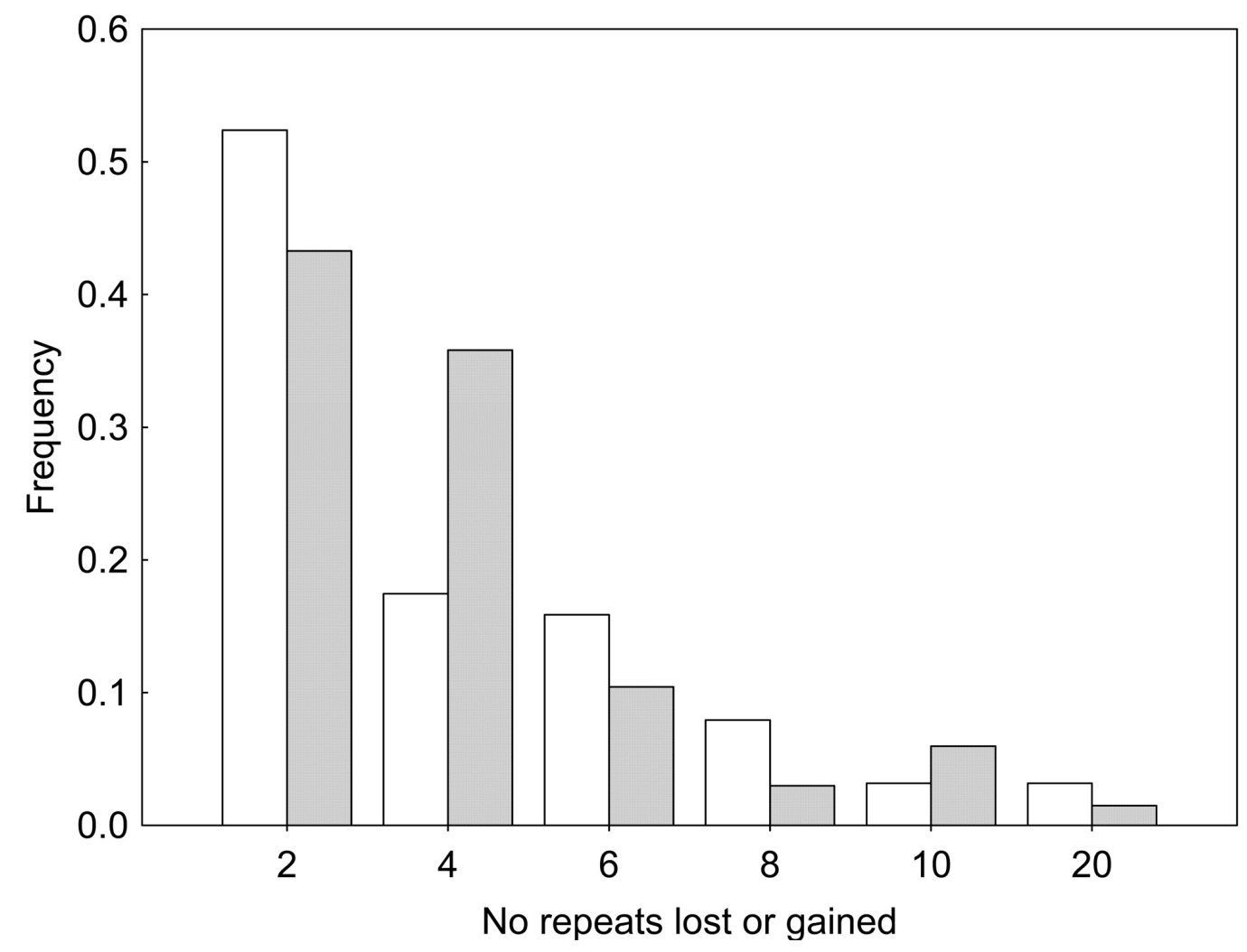


Similarly for the sperm tissue of control and test $F_{1}$ groups, the distribution of mutants did not significantly differ and thus data were combined (Table 3.1.8).

Table 3.1.8. ESTR mutation spectra in the $F_{1}$ offspring of control and treated males (sperm).

\begin{tabular}{|l|l|l|l|}
\hline Group & Gains (\%) & Loses (\%) & Total \\
\hline Control B & 14 & 5 & 19 \\
\hline Control C & 12 & 13 & 25 \\
\hline Control & $26(59.09 \%)$ & $18(40.91 \%)$ & 44 \\
\hline B+C & & 17 & \\
\hline Test B & 5 & 12 & 22 \\
\hline Test C & 17 & $29(56.86 \%)$ & 51 \\
\hline Test B+C & $22(46.14 \%)$ & $P=0.1209$ & \\
\hline$X^{2}, \mathrm{df}^{*}{ }^{*}$ & 2.40 & $47(49.47 \%)$ & 95 \\
\hline Total $^{*}$ & $48(50.53 \%)$ & $P=0.9183$ & \\
\hline$X^{2}, \mathrm{df}=1^{\dagger}$ & 0.01 & & \\
\hline
\end{tabular}

* Chi-square test for difference between groups

${ }^{\dagger}$ Chi-square test for homogeneity of total number of gains of losses

Table shows the incidence of unique mutations involving gain or loss of repeat units at the Ms6-hm locus detected in DNA samples extracted from sperm of the eight-week old first-generation offspring of control and treated males. The values of chi-square test for the differences between groups and homogeneity within group and corresponding probabilities are also shown. 
Even though the incidence of mutations involving gain or loss of repeat units was statistically indistinguishable between the control and test groups, the spectra of ESTR mutation significantly differed (Figure 3.1.9). This was attributed to the prevalence of large mutational changes detected in the $F_{1}$ test group.

Figure 3.1.9. Spectra of ESTR mutations in DNA samples extracted from sperm of $F_{1}$ males.

Graph shows the combined data for 8-week-old mice; and all mosaic mutants were treated as a single mutational event. The number of repeats lost or gained are binned as $\pm 2-3$ repeats $( \pm 1), \pm 4-5$ repeats $( \pm 1), \pm 6-7$ repeats $( \pm 1), \pm 8-9$ repeats $( \pm 1), \pm 10-19$ repeats $( \pm 9)$, and $\pm 20+$ repeats $( \pm 20)$. White bars represent control groups, while grey bars represent their age matched test groups. The frequency distributions of the two groups significantly differ (Kolomogorov-Smirnov two-sample test, $P=0.0351$ ) which would not be the case if a correction was applied for multiple testing.

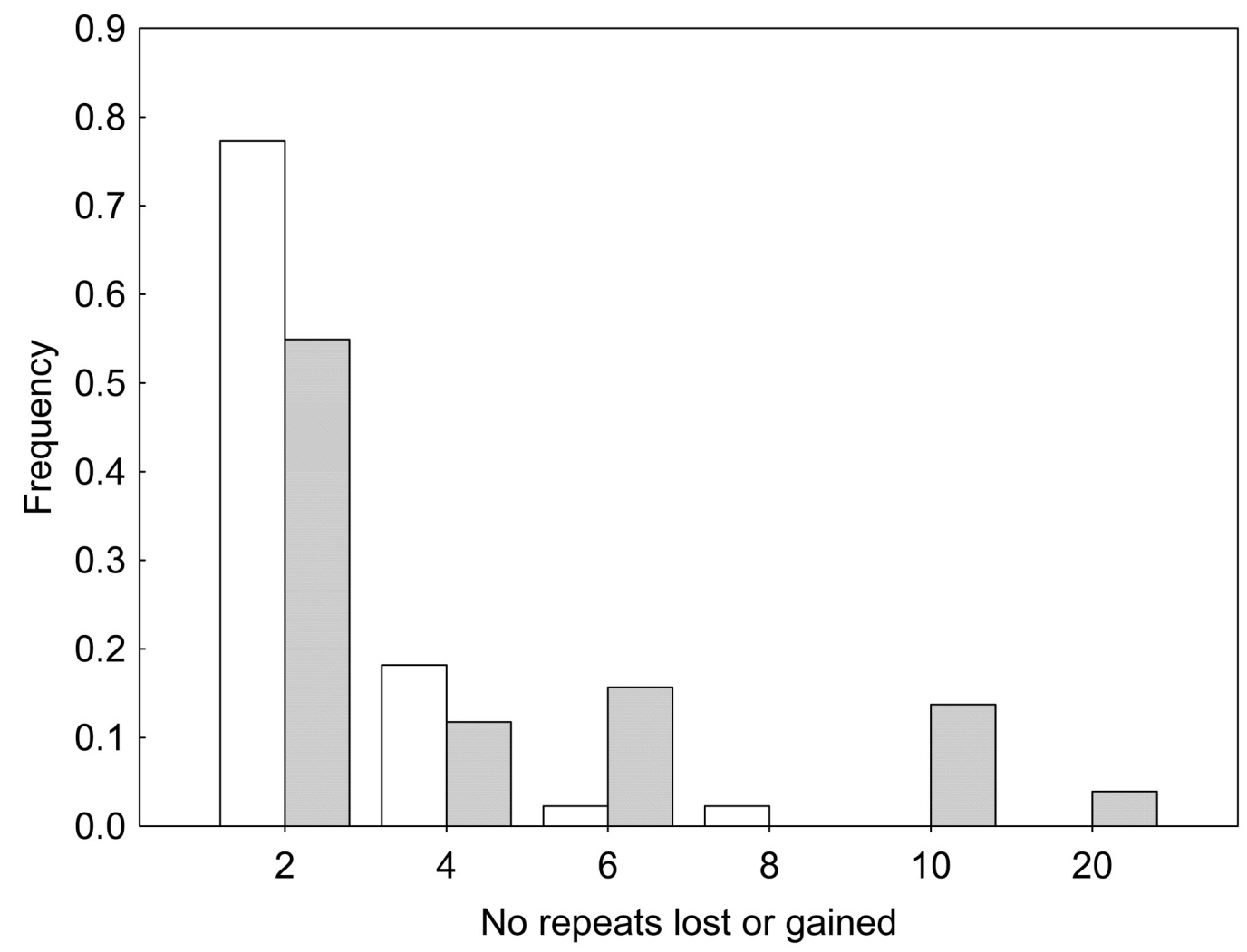


The spectra of ESTR mutation were also established in DNA samples extracted from the brain tissue of $F_{1}$ offspring of control males. As the distribution of mutants did not significantly differ in the brain tissue of control and test males, the data were combined. The incidence of gains and losses for DNA samples extracted from brain of all $F_{1}$ males did not show significant bias toward gains or losses (Table 3.1.9).

Table 3.1.9. ESTR mutation spectra in the $F_{1}$ offspring of control and treated males (brain).

\begin{tabular}{|l|l|l|l|}
\hline Group & Gains (\%) & Loses (\%) & Total \\
\hline & & & \\
\hline Control B & 7 & 11 & 17 \\
\hline Control C & 8 & 11 & 19 \\
\hline Control B+C & $15(41.67 \%)$ & $58.33(40.91 \%)$ & 36 \\
\hline Test B & 6 & 11 & 17 \\
\hline Test C & 6 & 10 & 16 \\
\hline Test B+C & $12(36.36 \%)$ & $21(63.64 \%)$ & 33 \\
\hline$X^{2}, \mathrm{df}=1^{*}$ & 0.20 & $P=0.6547$ & \\
\hline Total & $27(39.13 \%)$ & $42(60.87 \%)$ & 69 \\
\hline$X^{2}, \mathrm{df}=1^{\dagger}$ & 3.26 & $P=0.0701$ & \\
\hline
\end{tabular}

* Chi-square test for difference between groups

${ }^{\dagger}$ Chi-square test for homogeneity of total number of gains of losses

Table shows the incidence of unique mutations involving gain or loss of repeat units at the Ms6-hm locus detected in DNA samples extracted from brain of the eight-week old first-generation offspring of control and treated 
males. The values of chi-square test for the differences between groups and homogeneity within group and corresponding probabilities are also shown.

The spectrum of ESTR mutations in DNA samples extracted from brain of $F_{1}$ males is shown in Figure 3.1.10.

Figure 3.1.10. Spectra of ESTR mutations in DNA samples extracted from brain of $F_{1}$ males.

Graph shows the combined data for 8-week-old mice; and all mosaic mutants were treated as a single mutational event. The number of repeats lost or gained are binned as $\pm 2-3$ repeats $( \pm 1), \pm 4-5$ repeats $( \pm 1), \pm 6-7$ repeats $( \pm 1), \pm 8-9$ repeats $( \pm 1), \pm 10-19$ repeats $( \pm 9)$, and \pm 20 + repeats $( \pm 20)$. White bars represent control groups, while grey bars represent their age matched test groups. White bars represent the $F_{1}$ of control groups, while grey bars represent their age matched test groups. The frequency distributions of the two groups do not significantly differ (KolomogorovSmirnov two-sample test, $P=0.9997$ ).

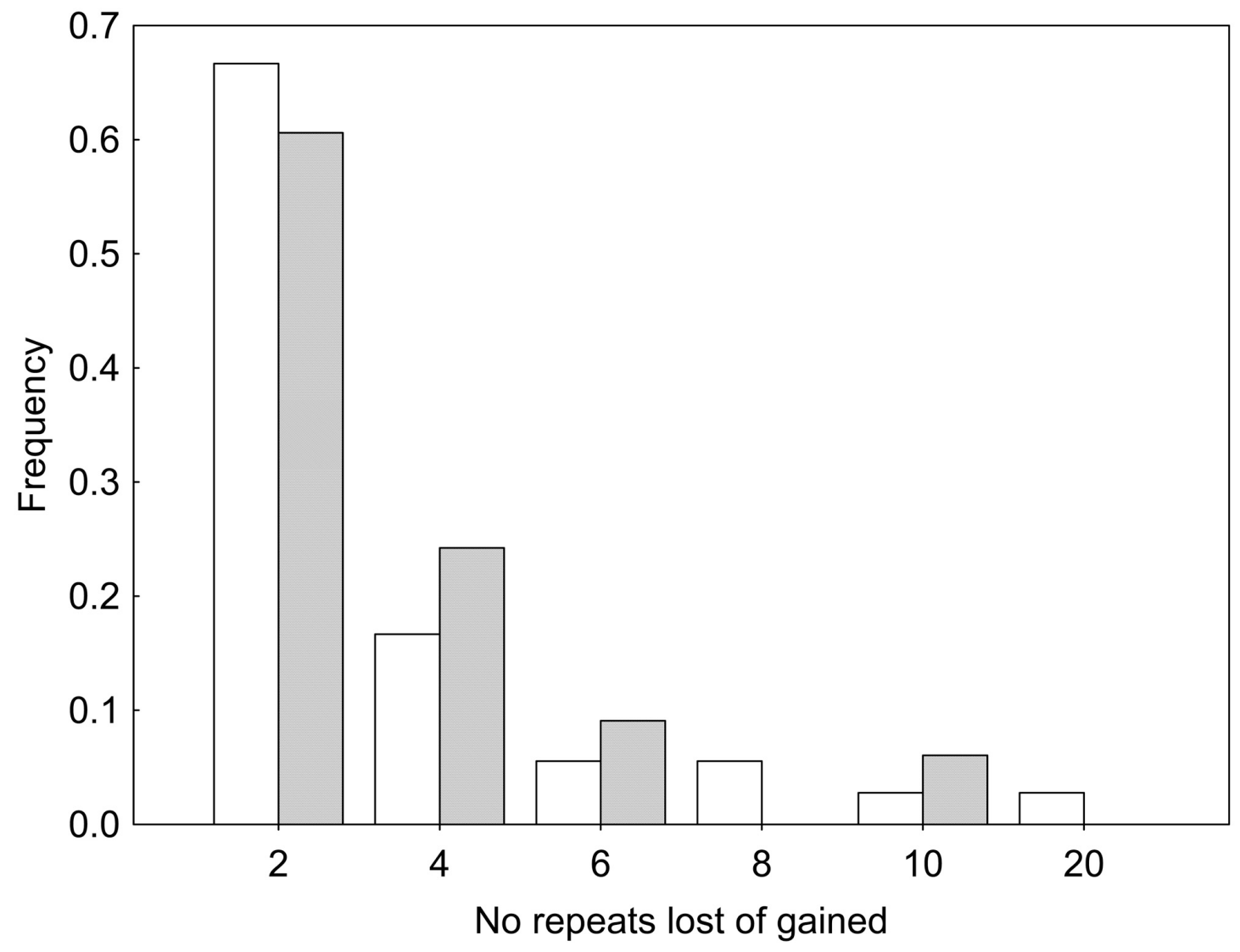


Since the incidence of mutations involving gain or loss of repeat units was statistically indistinguishable between brain and sperm tissue of all $F_{1}$ groups, the data were aggregated and the combined distributions of length changes were generated for all ESTR mutations, which did not significantly differ between groups (Table 3.1.10).

Table 3.1.10. ESTR mutation spectra in all studied groups.

\begin{tabular}{|l|l|l|l|}
\hline Group & Gains (\%) & Loses (\%) & Total \\
\hline Control B & 21 & 15 & 36 \\
\hline Control C & 20 & 24 & 44 \\
\hline Control B+C & $41(51.25 \%)$ & $39(48.75 \%)$ & 80 \\
\hline Test B & 11 & & 39 \\
\hline Test C & 23 & 28 & 45 \\
\hline Test B+C & $34(40.48 \%)$ & $50(59.52 \%)$ & 84 \\
\hline$X^{2}, \mathrm{df}=1^{*}$ & 1.92 & $P=0.1662$ & \\
\hline Total & $75(45.73 \%)$ & $89(54.27 \%)$ & 164 \\
\hline$X^{2}, \mathrm{df}^{*} 1^{\dagger}$ & 1.20 & $P=0.2743$ & \\
\hline
\end{tabular}

* Chi-square test for difference between groups

${ }^{\dagger}$ Chi-square test for homogeneity of total number of gains of losses

Table presents aggregated data for the types of ESTR mutations detected in all tissues. The values of chi-square test for the differences between groups and homogeneity within group and corresponding probabilities are shown. 
The overall spectrum of ESTR mutations in DNA samples extracted from both tissues of $F_{1}$ males is shown in Figure 3.1.11.

Figure 3.1.11. Spectra of ESTR mutations in DNA samples extracted from sperm and brain of $F_{1}$ males.

Graph shows the combined data for 8-week-old mice; and all mosaic mutants were treated as a single mutational event. The number of repeats lost or gained are binned as $\pm 2-3$ repeats $( \pm 1), \pm 4-5$ repeats $( \pm 1), \pm 6-7$ repeats ( \pm 1$), \pm 8-9$ repeats $( \pm 1), \pm 10-19$ repeats $( \pm 9)$, and $\pm 20+$ repeats $( \pm 20)$. White bars represent the $F_{1}$ of control groups, while grey bars represent their age matched test groups. The frequency distributions of the two groups do not significantly differ (Kolomogorov-Smirnov two-sample test, $P=0.2452$ ).

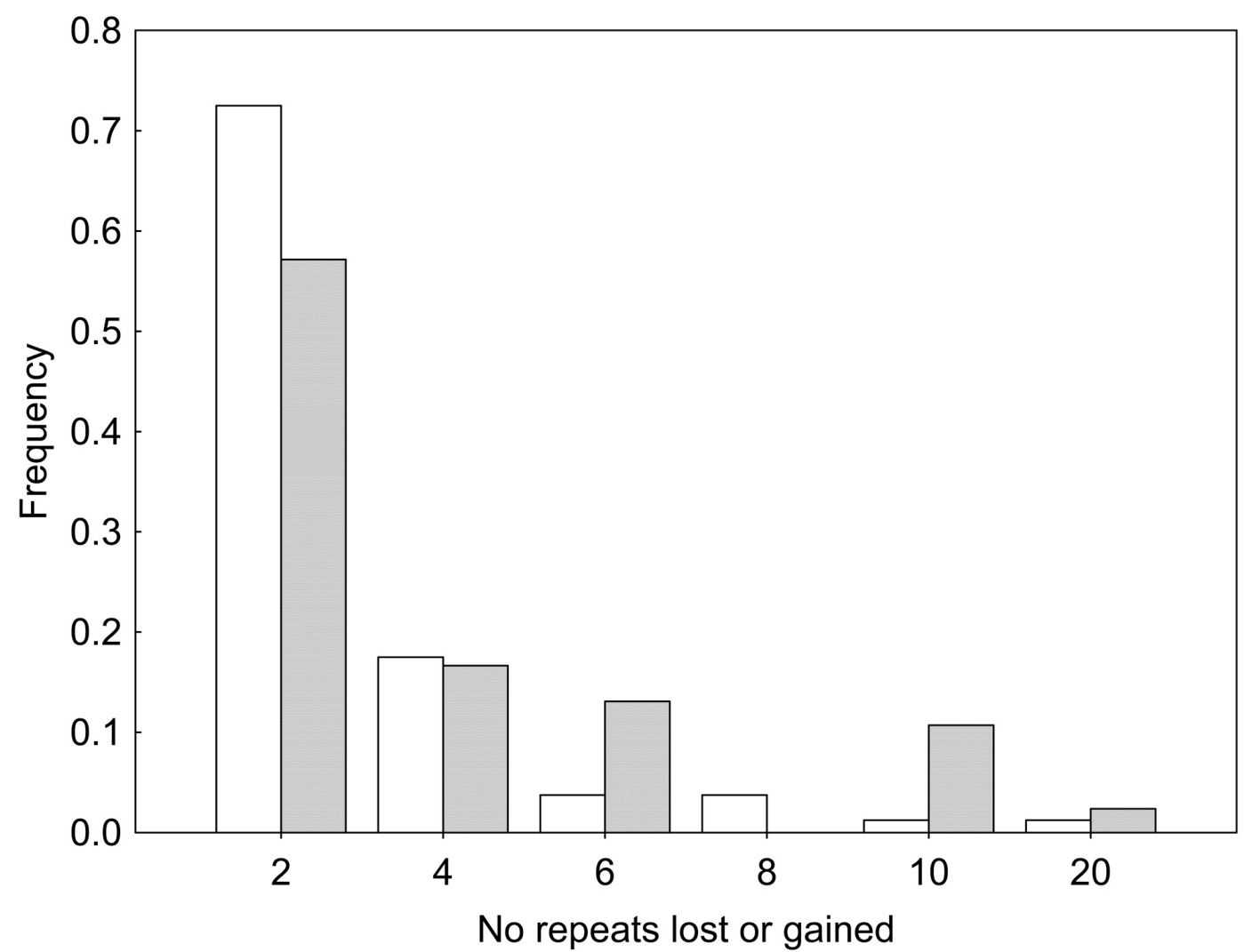

Similarly to the $F_{0}$ data, there the corresponding distributions did not significantly differ. Considering all the above data, it was therefore concluded that methyl-donor deficiency does not affect the spectra of ESTR mutation in the germline of directly treated male mice and their first-generation offspring. 


\subsection{Pig-A assay}

\subsubsection{Optimisation of method}

The analytical performance of the staining and data acquisition procedures were evaluated in an independent experiment where the technique was used by plotting unstained Balb/c mouse RBCs and then mixing it with a similar volume of stained naive population of RBCs. Three control males were used on each occasion and the technique was repeated with similar results. A preview of the recorded results is given below. As with the biological standards for instrument set up purposes, the unstained cells exhibited the GPI-anchor deficient phenotype, and their frequencies were determined via flow cytometric analysis as described in Section 2.2.2.4.

At the beginning of the experiment, unstained Balb/c mouse blood was subjected to fluorescent cytometric analyses in order to visualize the negalive cells.

Figure 3.2.1.1. Unstained RBC population. (a)

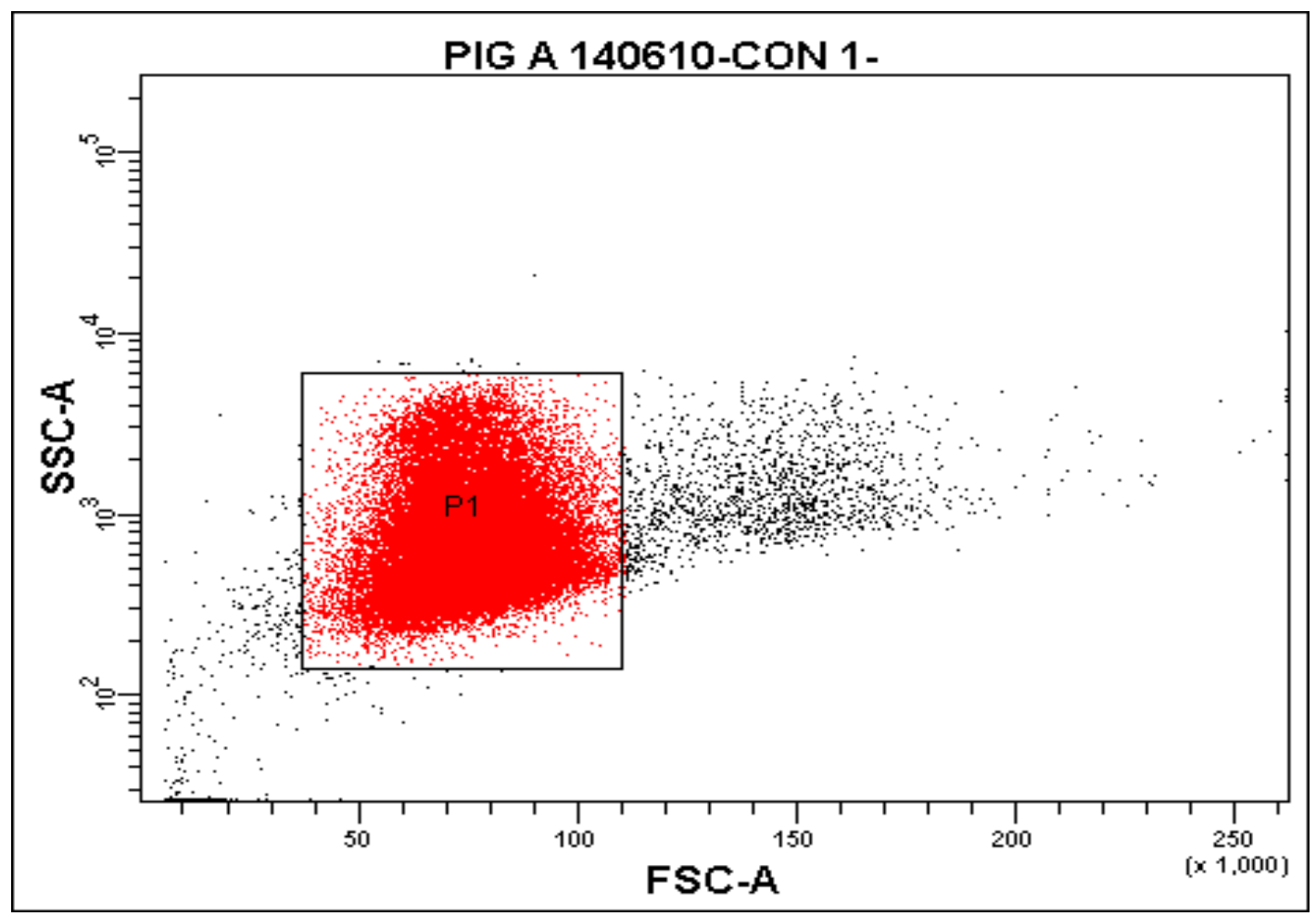


(b)

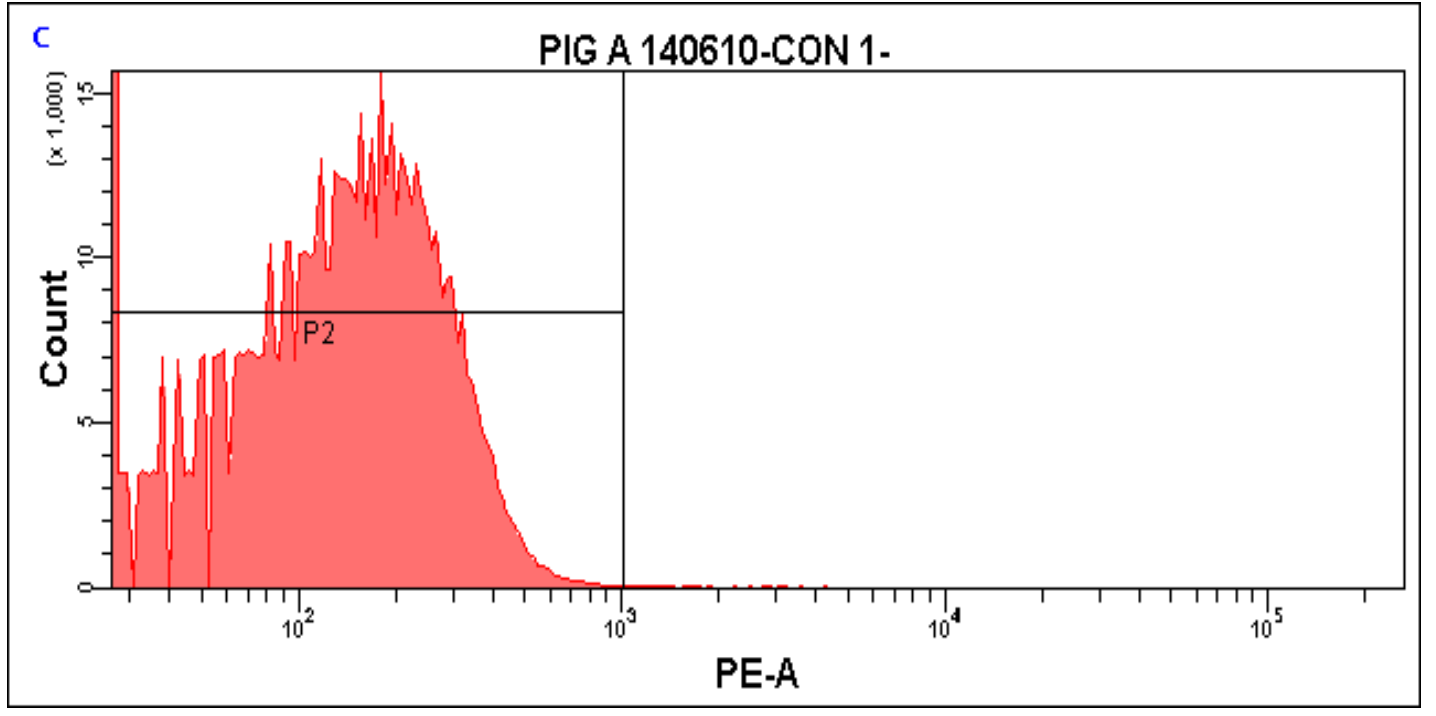

Figure 3.2.1.1 (a) Bivariate graph illustrates the gating approach for the mutant scoring application described here. Gated events (RBCs) are plotted on a side scatter versus a forward scatter. Side scatter gates cells according to their density while forward scatter gates cells according to their size.

Only RBCs are selected in this graph. Other events are excluded based on their failure to exhibit light scatter characteristics of cells (on the far left side- cell debris) or else their high fluorescence intensity (at the far right sidetwo or more red blood cells stuck together).

Thus while the lympholyte reagent physically removed the bulk of platelets and leukocytes, this gating strategy further ensures that mutant RBC frequencies generated by the right plot are not affected by these suspicious events.

Figure 3.2.1.1 (b) Gated events (RBCs) are plotted on a number of events (number of cells detected) versus their fluorescent emission.

A homogenous population of one million unstained events was recorded with a fluorescent height spanning form zero to $10^{3}$. The results of this analysis were recorded in Table 3.2.1.1 below. 
Table 3.2.1.1. Unstained RBC population.

\begin{tabular}{|llrr|}
\hline Experiment Nam... PIG A PE AQU & & \\
Specimen Name: & PIG A 140610 & & \\
Tube Name: & CON 1- & & \\
Record Date: & 14-Jun-2010 14:55:09 & & PE-A \\
& & & Mean \\
Population & \#Events & \%Parent & 78 \\
\hline P1 & $1,000,000$ & 96.6 & 78 \\
$\square$ P2 & 999,861 & 100.0 & \\
\hline
\end{tabular}

Stained blood was then added to the unstained population in an approximate rate of $1: 1$ in order to visualize the two distinct populations simultaneously and aid a rational and consistent set of the position of the vertical line that defines mutant versus non-mutant cells, as seen below in Figure 3.2.1.2.

Figure 3.2.1.2. Stained and unstained RBC population.

(a)

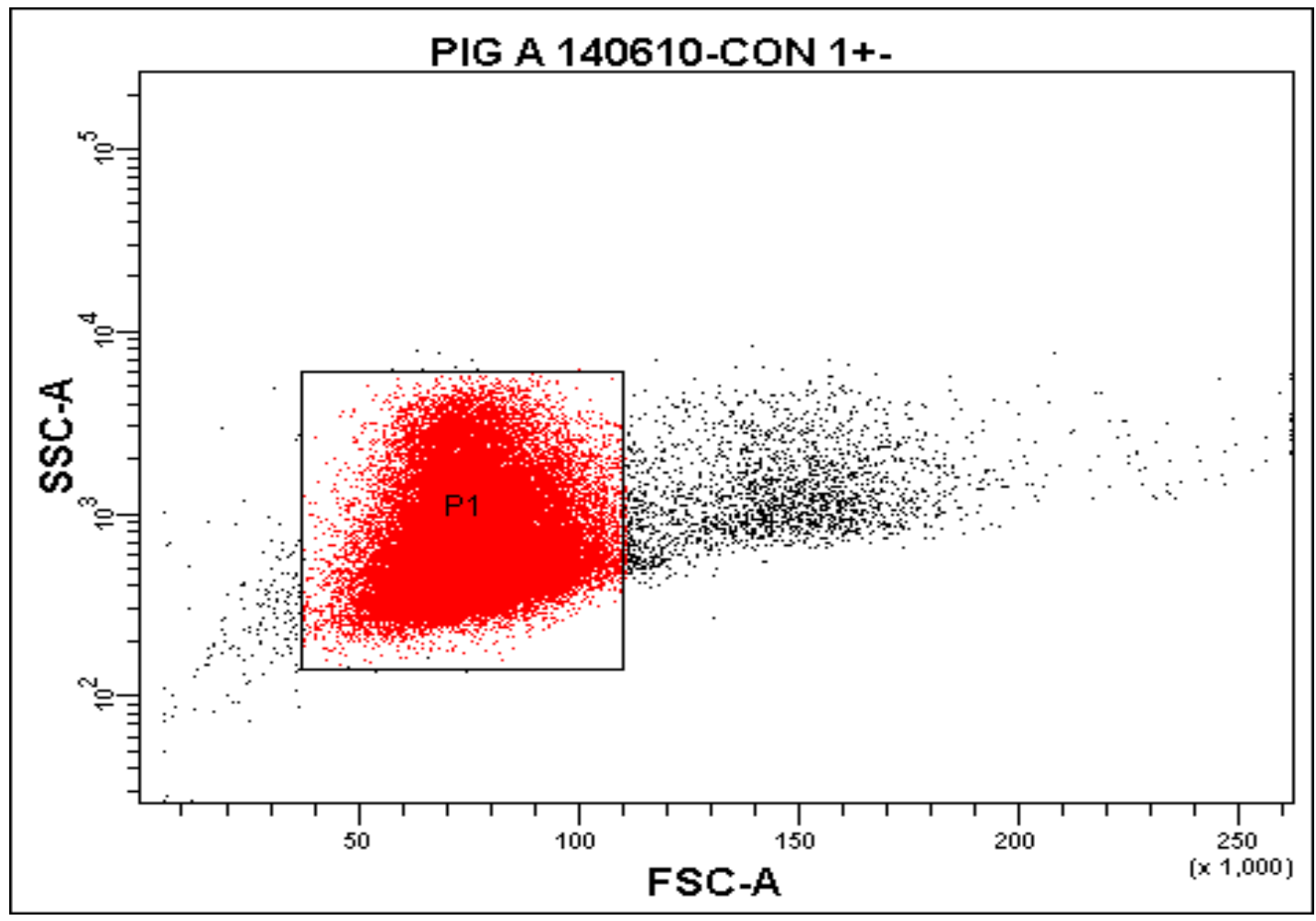


(b)

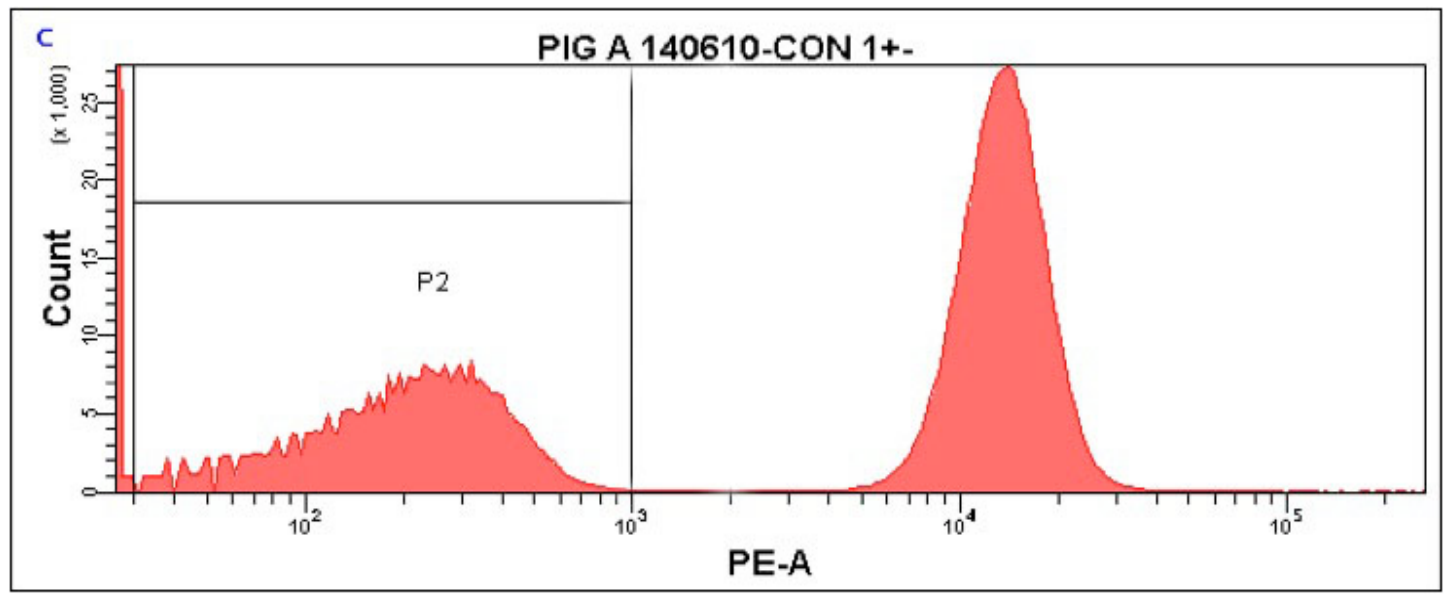

Figure 3.2.1.2 (a) Bivariate graph illustrates the mixed unstained and stained cell population. Gated events (RBCs) are plotted on a side scatter versus a forward scatter. Other events are excluded based on their failure to exhibit light scatter characteristics of RBCs cells. Figure 3.2.1.2 (b) Gated events (RBCs) are plotted on a number of events (number of cells detected) versus their fluorescent emission.

As seen in Figure 3.2.1.2 (b), after evaluation of the stained and unstained population, the position of the vertical line that defines mutant versus non-mutant cells was position is such way as all events recorded with a fluorescent height lower that $10^{3}$ are considered mutants whereas all events recorded higher than $10^{3}$ are considered normal RBCs.

A population of one million events was recorded and the results recorded in Table 3.2.1.2 below. 
Table 3.2.1.2. Mix of stained and unstained RBC population.

\begin{tabular}{|llrr|}
\hline $\begin{array}{l}\text { Experiment Nam... PIG A PE AQU } \\
\text { Specimen Name: }\end{array}$ PIG A 140610 & & \\
$\begin{array}{l}\text { Tube Name: } \\
\text { Record Date: }\end{array}$ & CON 1+- & & \\
& 14-Jun-2010 15:08:18 & & \\
Population & & & PE-A \\
\hline$\square$ P1 & \#Events & \%Parent & Mean \\
$\square$ P2 & $1,000,000$ & 95.7 & 7,840 \\
& 352,068 & 35.2 & 243 \\
\hline
\end{tabular}

The stained control mouse blood was then subjected to fluorescent cytometric analyses on its own, in order to record the number of mutation events expected in a normal red blood cell population (Figure 3.3.1.3).

Figure 3.3.1.3. Stained RBC population.

(a)

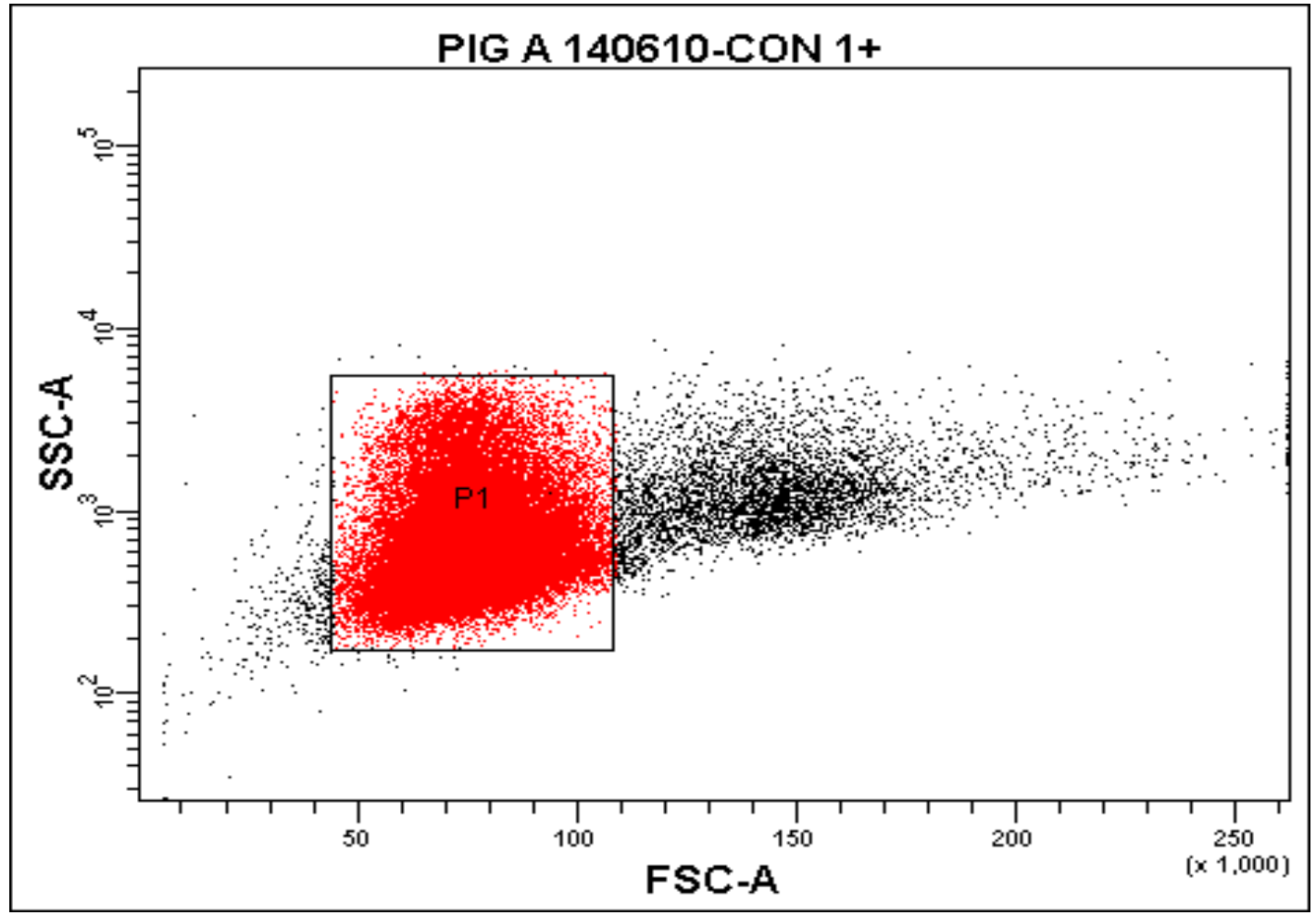


(b)

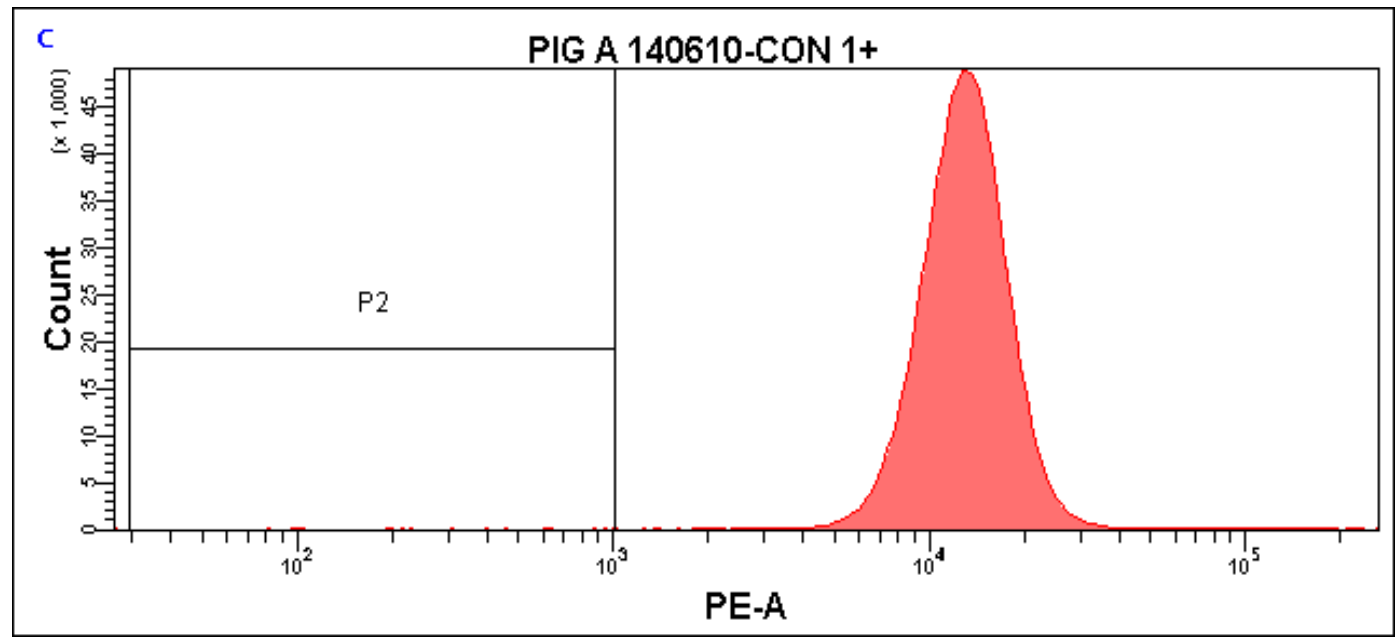

Figure 3.3.1.3 (a) Bivariate graph illustrates the gating approach for the mutant scoring application as described previously. Gated events (RBCs) are plotted on a side scatter versus a forward scatter. Other events are excluded based on their failure to exhibit light scatter characteristics of RBCs cells.

Figure 3.3.1.3 (b) Gated events (RBCs) are plotted on a number of events (number of cells detected) versus their fluorescent emission. A population of one million events was recorded. The vast majority of cells were stained (thus normal pig-a gene containing cells) which fluorescently emitted in approximately $10^{3}$.

A population of one million events was recorded, and seventeen events were recorded as mutants. Results were recorded in Table 3.2.1.3 below.

Table 3.2.1.3. Stained RBC population from control sample.

\begin{tabular}{|llrr|}
\hline Experiment Nam... PIG A PE AQU & & \\
Specimen Name: & PIG A 140610 & & \\
Tube Name: & CON"2+ & & \\
Record Date: & 14-Jun-2010 15:19:54 & & PE-A \\
& & & Mean \\
Population & \#Events & \%Parent & 26,846 \\
\hline P1 & 988,438 & 88.9 & 314 \\
$\square$ P2 & 32 & 0.0 & \\
\hline
\end{tabular}




\subsubsection{Effects of methyl deficient diet on Pig-A mutation rate}

To validate the technique for mutation at the Pig-A locus, blood samples were collected from 2 Balb/c male mice, which at the age of 4 weeks were injected intraperitonealy with $40 \mathrm{mg} / \mathrm{kg}$ of ethylnitrosourea (ENU) and culled 4 weeks later. Blood samples from those mice were compared with blood samples from the $F_{0}$ Control and Test groups as well as the blood samples form their $F_{1}$ progeny.

Table 3.2.2.1 presents a summary of the incidence of mutations involving the Pig-A gene, in RBCs of all ENU, Control and Treated groups.

Table 3.2.2.1. Summary of Pig-A mutational data.

\begin{tabular}{|c|c|c|c|c|}
\hline & \multicolumn{2}{|l|}{ Control } & \multicolumn{2}{|l|}{ Treated } \\
\hline Group, age & Animal & $\begin{array}{l}\text { No } \\
\text { mutations } \\
\quad \times 10^{-6}\end{array}$ & Animal & $\begin{array}{c}\text { No mutations } \\
\times 10^{-6}\end{array}$ \\
\hline ENU, 8 weeks & CON1 & 17 & ENU1 & 229 \\
\hline & CON2 & 32 & ENU2 & 213 \\
\hline & CON3 & 77 & & \\
\hline Mean \pm s.e.m. & & $42.00 \pm 18.03$ & & $221.00 \pm 8.00$ \\
\hline $\begin{array}{l}F_{0} \text { Diet } A \text {, } \\
12 \text { weeks }\end{array}$ & SUF6 & 1 & DEF6 & 5 \\
\hline & SUF7 & 1 & DEF7 & 9 \\
\hline & SUF8 & 4 & DEF8 & 11 \\
\hline Mean \pm s.e.m. & & $2.00 \pm 1.00$ & & $8.33 \pm 1.76$ \\
\hline $\begin{array}{l}F_{0} \text { Diet } B \text {, } \\
14 \text { weeks }\end{array}$ & SUF11 & 3 & DEF11 & 25 \\
\hline & SUF12 & 16 & DEF12 & 32 \\
\hline & SUF13 & 17 & DEF13 & 15 \\
\hline & SUF15 & 15 & DEF14 & 5 \\
\hline & & & DEF15 & 13 \\
\hline Mean \pm s.e.m. & & $12.75 \pm 3.28$ & & $18.00 \pm 4.73$ \\
\hline $\begin{array}{l}F_{0} \text { Diet } C, \\
20 \text { weeks }\end{array}$ & SUF16 & 9 & DEF16 & 15 \\
\hline
\end{tabular}




\begin{tabular}{|c|c|c|c|c|}
\hline & SUF17 & 15 & DEF18 & 7 \\
\hline & SUF18 & 5 & DEF19 & 29 \\
\hline & SUF19 & 19 & DEF20 & 96 \\
\hline & SUF20 & 6 & & \\
\hline Mean \pm s.e.m. & & $10.80 \pm 2.69$ & & $36.75 \pm 20.27$ \\
\hline $\begin{array}{l}F_{1} \text { Diet } B \\
8 \text { weeks }\end{array}$ & SUF11.a.1 & 35 & DEF12.a.1 & 42 \\
\hline & SUF12.b.1 & 18 & DEF13.b.1 & 22 \\
\hline & SUF13.b.1 & 27 & DEF14.a.1 & 54 \\
\hline & SUF14.b.1 & 55 & DEF14.b.1 & 30 \\
\hline & SUF15.a.3 & 43 & DEF14.b.2 & 12 \\
\hline Mean \pm s.e.m. & & $36.00 \pm 6.38$ & & $32.00 \pm 7.38$ \\
\hline $\begin{array}{l}F_{1} \text { Diet } C, \\
8 \text { weeks }\end{array}$ & SUF16.b.1 & 42 & DEF17.b.1 & 4 \\
\hline & SUF17.a.1 & 12 & DEF18.a.1 & 22 \\
\hline & SUF17.b.1 & 22 & DEF18.a.2 & 3 \\
\hline & SUF18.a.1 & 21 & DEF20.a.3 & 5 \\
\hline & SUF19.a.1 & 14 & & \\
\hline & SUF19.b.1 & 18 & & \\
\hline Mean \pm s.e.m. & & $21.50 \pm 4.40$ & & $8.50 \pm 4.52$ \\
\hline $\begin{array}{l}\text { Mean for all } \\
\text { controls, } n=26\end{array}$ & \multicolumn{4}{|l|}{$20.92 \pm 3.48$} \\
\hline $\begin{array}{l}\text { Mean for all } F_{0} \\
\text { Diet, } n=12\end{array}$ & \multicolumn{4}{|c|}{$21.83 \pm 7.23 ; x^{2}=0.1937, \mathrm{df}=1, P=0.6599^{*}$} \\
\hline $\begin{array}{l}\text { Mean for all } F_{1} \\
\text { Diet, } n=9\end{array}$ & \multicolumn{4}{|c|}{$21.56 \pm 5.96 ; \chi^{2}=2.8699 ; \mathrm{df}=1, P=0.2381^{*}$} \\
\hline $\begin{array}{l}\text { Mean for ENU- } \\
\text { treated, } n=2\end{array}$ & \multicolumn{4}{|c|}{$221.00 \pm 8.00 ; X^{2}=5.3852 ; \mathrm{df}=1, P=0.0203^{*}$} \\
\hline
\end{tabular}

"Probabilities for difference for controls, Mann-Whitney Test.

It is important to note that blood samples were collected from the same animals whose sperm (and brain tissue for all $F_{1}$ ) DNA were later analysed with the ESTR assay. 


\section{Figure 3.2.2.1. Pig-A means.}

Graph shows the pooled data for all $F_{0}$ and $F_{1}$ Control (pooled together), all $F_{0}$ Test and $F_{1}$ Test males, along with the positive ENU control data.

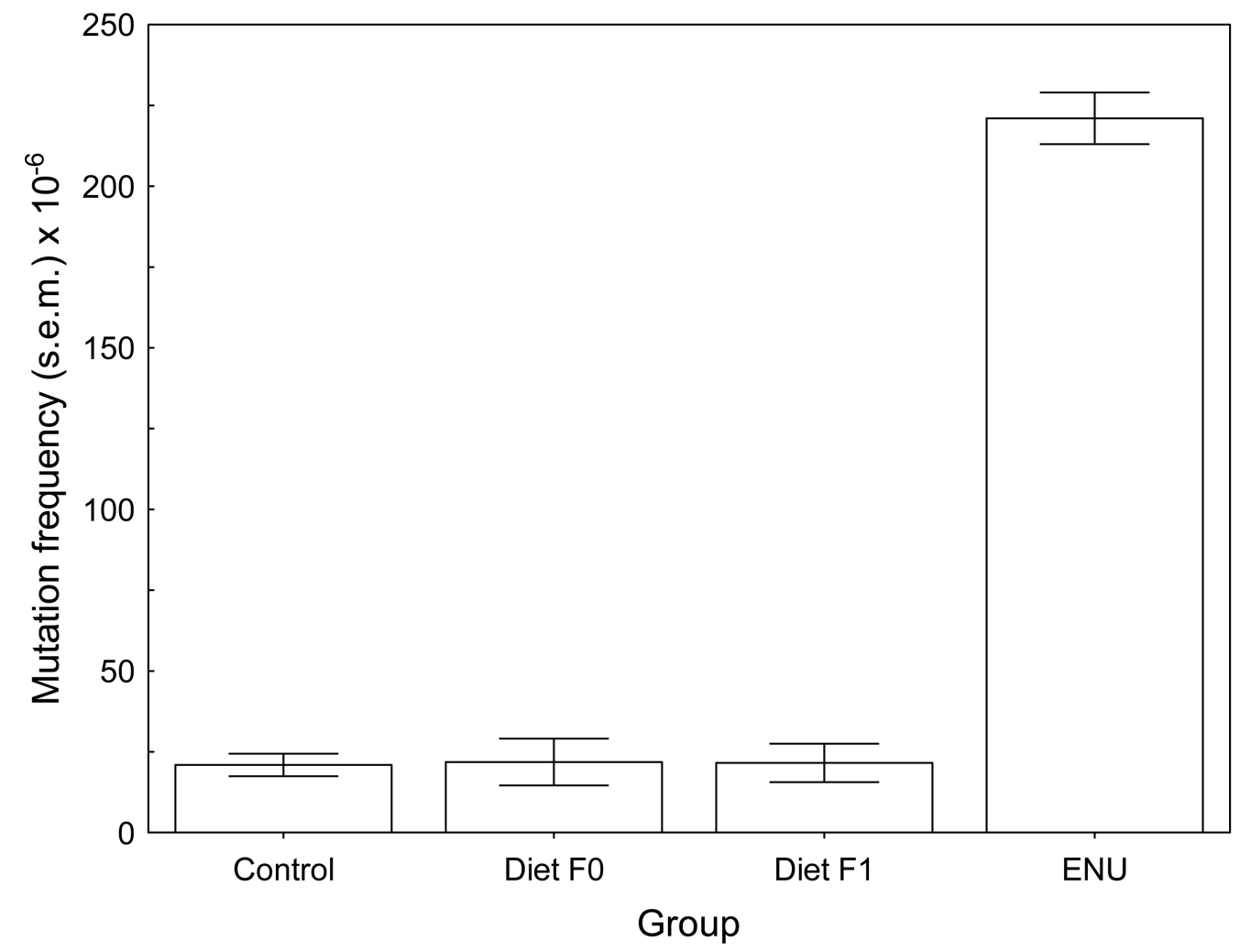

The results presented in this chapter show that exposure to wellrecognised mutagen ENU resulted in highly significant increase in the frequency of Pig-A mutations. In contrast, the analysis of treated mice, as well as their first-generation offspring failed to detect any significant dietattributer shifts in the frequency of Pig-A mutation.

\subsection{Phenotypic liver changes}

After 8 weeks on the methyl deficient diet, livers from the dissected Test $F_{0} A$ group were markedly pale in comparison with the livers of the dissected Control $F_{0} A$ group, which could be attributed to lipid accumulation, the hallmark of 'lipotropic' methyl-deficient diets as well as a broader stress to the specific organ caused by methyl-deprivation. 


\section{Chapter 4. Discussion}

\subsection{Summary of Results}

To date, fewer than 50 mutagens have been studied for their ability to cause heritable mutations, the majority of which are classical mutagens like radiation and anti-cancer drugs. However, little is yet known about the effects of diet on mutation induction in the mammalian germline (Swayne et al., 2012b). This thesis describes the study aimed to establish the long-term effects of a methyl donor deficiency on the genome stability of treated animals and their first-generation offspring.

To investigate the effects of methyl-deficient diet, BALB/c male mice were maintained for 8 weeks either on a methyl-deficient synthetic mouse diet or on control diet supplemented with choline and folic acid. To establish the impact of low dietary methyl-donor intake on the genome stability, the frequency of ESTR mutation was established in DNA samples extracted from sperm of treated animals, sacrificed either immediately after treatment, or 6 and 10 weeks after the end of dietary restriction. To establish whether paternal history of a low methyl-donor intake could result in transgenerational instability, the frequency of ESTR mutation was evaluated in DNA samples extracted from sperm and brain of eight-week-old $F_{1}$ offspring of treated males.

Using SM-PCR, the frequency of mutation at the Ms6-hm ESTR locus was analysed in the germline of directly treated mice. The same technique was used to establish whether the paternal exposure to a methyl-deficient diet could result in the transgenerational effects observed in the firstgeneration offspring. 
The results of this study show that an 8-week low dietary methyl-donor intake does not significantly affect ESTR mutation rate in the germline of treated males. The power calculations used in this study, based on the Poisson approximation, show that depending on the sample size, a 1.3-1.5 fold increase in ESTR mutation frequencies in the germline of all treated males could have been detected with an $\alpha=0.05$ level of significance. It was therefore concluded that methyl-donor deficiency causes negligible mutagenic effects on the mutation induction in the germline of directly treated animals (Voutounou et al., 2012). Likewise, 1.5-fold change in mutation frequencies could have also been detected in DNA samples extracted from brain and sperm of the $F_{1}$ of treated males, thus indicating the lack of measurable transgenerational effects of paternal dieting (Voutounou et al., 2012).

\subsection{Effects of diet on genome stability}

Epidemiological and experimental studies have shown that folate deficiency can result in elevated cancer risk (Ames \& Wakimoto, 2002; Kim, 2004; Pogribny et al., 2008). Since the accumulation of mutations represents one of the key steps of tumor progression (Loeb et al., 2003), a detailed analysis of the mutagenic effects of dietary restriction could provide significant evidence in an effort to elucidate the still poorly understood mechanisms underlying dietary-related carcinogenesis (Voutounou et al., 2012).

The mechanisms linking folate and the initiation of cancer formation remain unclear. Folate is involved in numerous essential single-carbon 
transfer reactions, necessary for the conversion of dUMP to dTMP (via Thymidilate synthase), functions as a coenzyme in purine synthesis, DNA synthesis and repair, and synthesis of methionine and SAM; necessary for DNA methylation.

There are two distinct pathways by which folate deficiency is believed to impact genome stability and lead to tumorigenesis (Figure 1.3.1). The first potential tumorigenic pathway of folate deficiency can be attributed to uracil misincorporation during DNA replication (Blount et al., 1997). Folic acid and its metabolites are involved in nucleotide synthesis (Section 1.1.4), and it has been shown that prolonged folate depletion causes disparity in deoxynucleotide pools by decreasing the intracellular levels of tetrahydropholate, which creates an imbalance in nucleotide precursors (increase in dUTP/dTTP ratios), leading to error-prone DNA synthesis such as misincorporation of uracil (Section 1.1.5) into DNA (Blount et al., 1997). The resultant U:A base pairs are potentially mutagenic because of secondary repair activity that can generate abasic sites (Auerbach et al., 2005), creating point mutations or chromosomal breaks at T:A base pairs (Linhart et al., 2009). Subsequently, folate deficiency is believed to dramatically decrease DNA repair efficiency as it disturbs nucleotide syntheses, promoting DNA damage, via chromosomal breakage (Sibany et al., 2002; Zhang et al., 2003).

Fenech and co-authors linked chromosomal damage and DNA methylation changes with folate deficiency (Fenech et al., 2001). Ames compared this type of DNA damage with the DNA damage induced by ionizing radiation and linked it to the promotion of carcinogenesis (Ames, 
2001). A follow up study by Ames and Wakimoto highlighted the role of diet in cancer prevention (Ames \& Wakimoto, 2002) and subsequent studies conducted by Kim and co-authors and Pogribny and co-authors revealed that the mechanism(s) resulting in elevated cancer risk following a low dietary folate intake are epigenetic, even though they continue to remain elusive (Kim et al., 2004; Pogribny et al., 2008). Pogribny and co-authors further reported that DNA isolated from animals fed with methyl-deficient diets (containing low levels of folic acid or vitamin B12), is characterized by a greater level of uracil misincorporation (Pogribny et al., 2012).

Epidemiological studies have associated deficiency in the B-vitamin folate and an increase in cancer risk, particularly for colorectal cancer (Giovannucci et al., 1998; Jacobs et al., 2003), breast cancer (Ericson et al., 2007), and pancreatic cancer (Larsson et al., 2006). Fenech recently reviewed the findings of numerous in vitro and in vivo studies in rodents and humans associated with folate and its function in the maintenance of nuclear and mitochondrial genome integrity, and concluded that folate deficiency can destabilise genomes of somatic cells, leading to chromosome damage (Fenech, 2012).

Nevertheless, the results of abovementioned studies on the elevated frequency of chromosome aberrations do not necessarily imply that the mutagenicity caused by folate-deficient diets is adequate to enhance tumor development. The reason behind this could be the fact that tumor progression is mainly attributed to the accumulation of point mutations, which in the case of this study we failed to detect (Voutounou et al., 2012). The results in this study are in line with those obtained by Linhart and co-authors 
on wild-type mice that were placed on a folate deficient diet for a duration of 8 months. The authors found that folate deficiency induced genomic uracil misincorporation and hypomethylation but did not increase DNA point mutations, indicating that the effects of a low-folate diet on DNA methylation and point mutations are insufficient to promote tumor development (Linhart et al., 2009). Moreover, Van Guelpen and co-authors reported that folate deficiency can decrease the risk of intestinal tumor (Van Guelpen et al., 2006), and according to some recent epidemiological studies, folate supplementation either had no effect (Logan et al., 2008) or increased the risk of intestinal tumors (Cole et al., 2007).

Even though folate deficiency has been reported to increase DNA strand breaks (Blount et al., 1997; Kim et al., 2000), direct proof of mutations by sequence analysis is missing and therefore the importance of uracil misincorporation into DNA as a mutagenic mechanism still remains unclear (Krokan et al., 2002). There is a possibility that uracil-related DNA strand breaks inhibit transcription of tumor-relevant genes without causing DNA mutations, which remains to be addressed (Kim et al., 2000). The in vivo study conducted by Linhart and co-authors, mentioned above, aimed to establish whether chronic folate deficiency alone or with additional mutations in the DNA repair pathway may affect the frequency and spectra of mutations in non-tumor tissues. The genetic effects of folate deficiency were examined after a subjection of 3-month-old wild type and uracil DNA glycosylase (Ung) knockout mice $\left(\mathrm{Ung}^{-1-}\right)$ to an 8-month folate-deficient diet (Linhart et al., 2009). Ung is an excision repair enzyme that removes misincorporated uracil 
during DNA replication (Nilsen et al., 2000) and repairs U:G mispairs that occur as a result of cytosine deamination (An et al., 2005).

This study showed that folate deficiency resulted in a $24 \%$ increase of the uracil content in colon epithelial cells in both wild-type and $\mathrm{Ung}^{-1-}$ mice, which was attributed to uracil misincorporation. On the other hand, the data on the effects of folate and Ung deficiency on point mutations showed that folate deficiency had no significant effect on the frequency or spectrum of point mutations on wild-type or Ung ${ }^{-/}$mice. In particular, the study failed to detect an increase in point mutations at T:A base pairs, resulting from repair of uracil misincorporation (Linhart et al., 2009). The authors concluded that although folate deficiency causes an increase in genomic uracil content through uracil misincorporation, it does not lead to an increase in DNA point mutations; nor does it increase transition mutations at $\mathrm{CpG}$ sites. The authors also stated that the effects of isolated folate deficiency on DNA point mutations are not sufficient to promote tumor formation, even in the presence of genetic susceptibility such as Ung deficiency (Linhart et al., 2009).

A study conducted by Swayne and co-authors in 2012 aimed to investigate the effect of folic acid on chromosomal damage in erythrocyte progenitor BALB/c mice cells (Swayne et al, 2012a). Since folate deficiency results in DNA hypomethylation, it was hypothesized that hypomethylation of pericentromeric DNA can cause centromere dysfunction and thus produce micronuclei due to chromosome loss (Fenech et al., 2011). In this study, male mice were weaned and fed (for 12 weeks) on a folic acid-deficient or control or folate-supplemented diet. The study reports a $24 \%$ increase in erythrocyte micronucleus frequency in the folate-deficient group compared 
with the mice kept on the control and supplemented diets. In contrast, the study failed to find diet-dependent significant differences in bone marrow uracil content, nor differences in global DNA methylation status between the three groups. The findings suggest that uracil misincorporation into DNA or DNA methylation perturbations caused by a folate deficient diet are either not involved in the process of micronuclues formation or that these DNA modifications are transient (Swayne et al, 2012a).

Another study by Swayne and co-authors was designed to establish the effects of folic acid-deficient diet in early development (in utero and during lactation) or post-weaning for 15 weeks on adult male Balb/c mice (Swayne et al., 2012b). Its results showed that sperm ESTR mutation frequency was increased 2-fold in male offspring weaned to the folic acid deficient diet, with a recorded $55 \%$ decrease in red blood cell (RBC) folate, decreased cauda sperm numbers and a higher percentage of sperm with abnormal DNA fragmentation index (\% DFI) compared to control mice. None of these effects were recorded in the male offspring weaned to the supplemented diet or the folic acid deficient or supplemented diets in early development (Swayne et al., 2012b). The authors concluded that early developmental deficient or supplemented diets had no effect on sperm numbers, or genome stability, while a 15 week folic acid deficiency in adult male mice caused a reduction in sperm numbers, an increase in germline chromatin damage and DNA mutation (Swayne et al., 2012b). The discrepancies between the Swayne experiment and the findings from this study could be explained by the use of different diets, the different 
developmental time periods examined and the fact that the Swayne study was delivered over a significantly longer period of time (15 weeks).

\subsection{Effects of diet on methylation status}

Although epigenetic effects of folate are not a focus on this project, it is important to note the role of methyl donors on gene expression, particularly given that folate deficiency can substantially affect the pattern of DNA methylation, including the sites involved in the regulation of transcription.

DNA methylation is essential for the regulation of numerous biological processes such as genomic imprinting, $\mathrm{X}$ chromosome inactivation, silencing of tumor suppressor genes, and repression of retroviral elements (Section 1.2.1.). It is believed that aberrant methylation patterns can be implicated in tumorigenesis by causing genomic instability, abnormal imprinting, and deregulated expression of oncogenes or tumor suppressor genes (Zhu et al., 2006). It is well established that epigenetic changes play a major role in predisposing organisms to cancer/minor effects of destabilization.

Several recent human and animal studies have shown that the increase in cancer risk is caused by the accumulation of DNA methylation errors, due to diets containing low levels of methyl donors such as methionine (Section 1.2.3), choline (Section 1.2.4) and folate (Section 1.2.5), which contribute to DNA methylation errors and are associated with increased cancer risk (Ames \& Wakimoto, 2002; Streffer et al., 2003; Kim, 2004; Pogribny et al., 2008).

Robertson and Wolffe established that methylation patterns are maintained through DNA replication by the activity of DNMT1 and one could 
hypothesise that folate deficiency can have a long-term impact on genome stability (Robertson \& Wolffe, 2000). As argued by Loeb and co-authors, the development of cancer is a multistep process in which somatic cells accumulate mutations in a specific clonal lineage, and thus it seems possible that hypomethylation following dietary restriction could destabilise the genome of treated individuals (Loeb et al., 2003).

Blount and co-authors investigated the potential effects of folate deficiency and found that observed changes in methylation patterns were only transient (Blount et al., 1997). Contradicting data from the Pogribny group showed prolonged DNA hypomethylation after a subjection of folate deficient diet in rodents (Pogribny et al., 2006). A study by McKay and coauthors examined the effects of low-folate diet on murine offspring exposed during gestation and lactation, and showed a decrease in global methylation status in the small intestinal tissue of the adult mice (McKay et al., 2011a). In contrast, a study of Ly and co-authors revealed a link between in utero folate supplementation and CpG hypomethylation of the mammary tissue among rat offspring of 28 weeks of age (Ly et al., 2011). Another study, by Sie and co-authors analyzed the effects of post-weaning folate supplementation equivalent to the recommended intake levels for women, revealed a decrease in global methylation levels in murine colorectal tissue (Sie et al, 2011). The results of these in vivo animal studies therefore demonstrate that both folate restriction and folate supplementation can lead to global DNA hypomethylation.

A number of studies employed a candidate-gene approach for the investigation of the effects of folate on DNA methylation. The chosen 
genomic loci are usually linked with a disease of interest, such as cancer or diabetes, for example the $\mathrm{N}$-acetyltransferase 2 (Nat2) gene, whose human homologue, NAT1, is associated with cancer development including breast carcinogenesis (Anderson et al., 2012). Wakefield and co-authors demonstrated that the Nat2 gene in adult rats exposed to a high-folate diet was under-expressed, demonstrating increase in methylation (Wakefield et al., 2010). Kim and co-authors evaluated the impact of folate deficiency on the methylation status of the $p 53$ tumor suppressor gene in rats subjected to a low-folate diet (Kim et al., 1997). The authors showed that the two-exon regions of the $p 53$ gene were hypomethylated in liver of treated animals. The results of this study suggest that methylation status could play a key role in the molecular underpinnings of carcinogenesis (Kim et al., 1997).

Burger and co-authors examined the impact of folic acid supplementation on the promoter regions of insulin receptor, PPAR- $\alpha$ and glucocorticoid receptor (genes involved in metabolic homeostasis) and found an increase in methylation in the promoter regions of these genes, compared to offspring exposed to the same in utero environment with the exception of a folate-adequate diet during puberty (Burdge et al., 2009).

McKay and co-authors examined the link between folate depletion during gestation and lactation with the methylation status at several loci involved in colorectal cancer. The data revealed distinct alterations in DNA methylation at these loci, in both wild-type and $\mathrm{Apc}^{+/ \mathrm{Min}}$ rats, at the stages of weaning and during adulthood (McKay et al., 2011b). The results of this experiment showed that the same folate exposure alters DNA methylation in a gene- and tissue-specific manner at different life stages. It was also 
demonstrated that differences in methylation were dependent on sex and genotype (Anderson et al., 2012).

Recent trials on humans revealed that folate supplementation either had no effect (Logan et al., 2008) or increased the risk of intestinal tumors (Cole et al., 2007). Another study showed that folate deficiency protects against intestinal tumor formation (Van Guelpen et al., 2006). Studies on isolated folate deficiency have shown conflicting results (Kim, 2005) ranging from a $20 \%$ decrease in DNA methylation (Balaghi, 1993), through no change (Le Leu et al., 2000), to a 56\% increase in global DNA methylation (Song et al., 2000).

The difficulty in interpreting these data comes from the fact that in some cases the tissues analyzed were such of low cellular turnover such as liver (Song et al., 2000a) or the periods of dietary intervention were considered short (Le Leu et al., 2000), even though a decrease in global DNA methylation resulting from nutrient deficiency is considered a passive process, that requires multiple cell divisions (Linhart et al., 2009).

The evidence from human, animal and in vitro studies points to the direction that the epigenetic effects of folate deficiency on DNA methylation are highly complex. The observed effects appear to be gene- and tissuespecific and also depend on the stage of exposure. There are also a number of other factors, including the severity and duration of diet intervention, as well interaction between the availability of methyl donors and other components of diet. Finally, the epigenetic effects of folate deficiency can be substantially modified by the presence of genetic variants affecting the folate metabolic pathways (Ly et al., 2012). 


\subsection{Analysis of results}

The mechanism underlying the effects of methyl-donor deficiency on the risk of cancer still remains poorly understood, which is mainly attributed to the existing controversy regarding the mutagenic potential of such intervention (Kim, 1999; Eichholzer et al., 2001). The purpose of this study was therefore to establish the long-term effects of methyl-donor deficient diet on the genome stability of treated animals and their first-generation offspring.

It is well documented that DNA damage in sperm can lead to impaired fertility or adverse effects on early embryonic development (Singer \& Yauk, 2010). Any amount of DNA damage that becomes fixed as mutations in sperm DNA may well become hereditary and therefore result in a range of genetic disorders (Swayne et al., 2012b). Since germ cell mutations are very rare (approximately $1.1 \times 10^{-8}$ per nucleotide per haploid genome), recent efforts have focused on quantifying mutation rates in highly variable noncoding regions of the genome (Roach et al., 2010).

Germ cell mutation frequencies can be measured at the mouse expanded simple tandem repeat (ESTR) loci, which are short (4-6bp) repeat units, tandemly repeated to form long homogeneous arrays up to $20 \mathrm{~kb}$ in length (Yauk, 2004; Yauk et al., 2008). ESTRs have a mutation rate a thousand times higher than normal protein coding genes, both in somatic and germ cells, providing a highly sensitive tool for the investigation of heritable mutation induction in laboratory mice. ESTRs further allow the determination of mutation frequencies at environmentally relevant doses using small sample sizes (Dubrova, 2005). Even though ESTR sequences are part of the non-coding regions of DNA, the doubling dose for ESTR mutations is similar 
to that of coding DNA (Somers et al., 2002), and these loci show a doseresponse relationship with both ionizing radiation (Barber et al., 2000; Dubrova et al., 1998; Dubrova et al., 2000a), chemical mutagens (Somers et al., 2002; Vilarino-Guell et al., 2003) and anticancer drugs (Glen et al., 2008). Therefore ESTRs provide an efficient way to survey the ability of agents to cause mutations and genetic instability in the mouse germline (Swayne et al., 2012b).

The reasoning for measuring the impact of methyl-donor deficiency on the mutation frequency exclusively in the male germline comes from Barber and co-authors, demonstrating that the $F_{1}$ progeny of female mice exposed to ionising radiation do not show elevation in their ESTR mutation frequencies (Barber et al., 2009). This is believed to occur due to the differences in the demethylation processes that take place during the embryonic stages between the male and female pronuclei as demonstrated by Hales and co-authors (Hales et al., 2011). Since in theory, the mechanism involved in DNA methylation should be similarly affected by ionising radiation and methyl-donor deficiency, the findings from the studies mentioned above suggest that observing an effect in the $F_{1}$ of methyl-donor deficient females is highly improbable. Therefore the experiment was restricted to the male germline. 


\subsubsection{ESTR mutation frequencies in control groups}

The first objective of this project was to determine the ESTR mutation frequencies in the germline of male mice kept on a control diet (Table 5.1.1) supplemented with choline and folic acid for a duration of 8,14 and 18 weeks and their 8 week old $F_{1}$ generation, all matched by age to the treated groups (Table 3.1). Figure 3.6 presents the mean ESTR frequencies in sperm and brain of all control males. As the frequencies did not significantly differ for all pair wise comparisons, control data was combined for each tissue (Tables 3.2 - 3.4).

The results from the control groups correlate with the findings of Hardwick and co-authors showing that within a group of young 12-week-old males, the frequencies of ESTR mutation across all tissues does not significantly differ. On the contrary for animals older than 48 weeks of age, the authors detected a highly significant elevation in sperm mutation frequency, whereas in brain, it remained unchanged (Hardwick et al., 2009).

This could be explained by the fact that the adult mouse brain consists of approximately $10^{8}$ cells (Williams, 2000) with a very low rate of cell proliferation (Hardwick et al., 2009). On the contrary, the total population of stem cells in the mouse testis consists of $70 \times 10^{3}$ cells (Tegelenbosch \& De Rooij, 1993). Male mice become sexually mature at the age of approximately 35 days and from that point, the undifferentiated $A_{s}$ spermatogonia undergo one mitotic division per approximately 2.8 days (De Rooij \& Russell, 2000). During spermatogenesis, each spermatogonium undergoes 8 rounds of DNA replication before differentiating into spermatocytes (De Rooij \& Russell, 2000). These data indicate that the total number of cell divisions that occur 
from early embryonic development until the age of $8,12,18$ and 22 weeks are approximately 32, 43, 56 and 66 respectively (Hardwick et al., 2009).

\subsubsection{Effects of methyl-donor deficiency on ESTR mutation induction}

The second objective of this study was to determine if a period of 8 weeks on a methyl-donor deficient diet could result in long-term effects on genome stability in the germline of male mice and if those effects could be later reversed by the methyl-adequate diet.

To address this, male mice were sampled directly after the end of treatment and again 6 and 10 weeks after the end of dietary restriction. Given that ESTR mutation is a replication-based process involving polymerase slippage, there should be a relationship between the accumulation of mutation and mitotic index (Yauk, 2004; Yauk et al., 2008). If methyl-deficient diet resulted in long-term genome destabilisation, then ongoing genomic instability would lead to the accumulation of mutations in tissues with a high mitotic index such as spermatogonia. By profiling mice over a considerable period of time following treatment, the contribution of ongoing genomic instability to the dynamics of ESTR mutation accumulation could thus be established.

Since ESTR mutations occur via replication slippage during DNA repair or replication processes at the pre-meiotic stages of spermatogenesis (Yauk, 2004; Yauk et al., 2008) it was anticipated that the final 4 weeks prior to sample collection for ESTR analysis would not affect the mutation outcome (Swayne et al., 2012b). The experimental design of this project is believed to effectively capture sperm derived from spermatogonial stem cells exposed to 
8 weeks of methyl-donor deficiency (Test $F_{0} A$ ), and a subsequent of 2 (Test $F_{0} B$ ) or 6 (Test $F_{0} C$ ) weeks of supplementation for germ cells committed to spermatogenesis, including 4 weeks as pre-meiotic spermatocytes (Voutounou et al., 2012).

Table 3.5 presents a summary of the ESTR mutation data. The student's t- test was used to determine if a statistically significant difference in mutation frequencies had been observed between the control and treatment mice, but the frequency of ESTR mutation in the germline of treated males did not significantly differ from that in the total control group. A comparison of ESTR mutation frequencies between the exposed and agematched groups did not also reveal any significant differences (Figure 3.7). Therefore, the data suggest that the effects of a methyl-donor deficiency on ESTR mutation induction in the germline of treated male mice are likely to negligible and that methyl-donor deficiency does not cause long-term genome instability in the germline of male mice.

When Pogribny and co-authors investigated the effects of folate deficiency on long-term changes to DNA methylation patterns in 2006, they used longer dietary periods $(9,18,24$ and 36 weeks) rather than the 8-week period used in this study. The Pogribny experiment was conducted using rats instead of mice and they were returned to a folate sufficient diet for a total of 54 weeks after the end of treatment. Their data revealed a global DNA hypomethylation in mice from all time frames, even though rats treated with the folate deficient diet for the shortest amount of time (9 weeks) were found to revert back to normal DNA methylation patterns after their return to the sufficient diet. For all other groups, the folate deficient diet led to long term 
changes to their DNA methylation patterns (Pogribny et al., 2006). It can be thus argued that since the 8-week period of methyl-deficiency analyzed in this study was considerably shorter than that in the Pogribny study, which could potentially explain the negative findings.

However, as Pogribny and co-authors observed a transient effect after a similar time period, short-term changes to mutation frequency should still have been detected in animals culled directly after treatment (Test $\left.F_{0} A\right)$ and those turned to a control diet for a short period of time after the end of treatment $\left(T e s t F_{0} B\right)$. The results therefore suggest that the 8-week period of dietary intervention was sufficient for the detection of the immediate effects on mutation frequency.

As mentioned above, the very high sensitivity of ESTR loci for monitoring germline mutation induction has previously been demonstrated in numerous publications (Dubrova et al., 1998; Barber et al., 2000; Dubrova et al., 2000; Vilarino-Guell et al., 2003; Glen et al., 2008). It therefore appears that if a methyl-donor deficient diet did cause long-term effects on mutation frequency in the male germline, we would have been able to detect it. Given this, the results presented here suggest that the 8-week period of methyldonor deficiency does not significantly alter ESTR mutation rate in the germline of treated animals. 


\subsubsection{Transgenerational effects of paternal methyl-donor deficiency}

So far, there is an accumulation of very strong evidence for transgenerational effects following paternal exposure to mutagens such as ionizing radiation, chemicals and anticancer drugs (Vilarino-Guell et al., 2003; Dubrova et al., 2000; Barber et al., 2006; Dubrova et al., 2008; Glen et al., 2008; Barber et al., 2009; Glen \& Dubrova, 2012) but the role of several nonmutagens that have been linked to genomic instability has not been thoroughly examined and thus remains poorly understood. Therefore, the third objective of this project was to analyze the effects of paternal dietary restriction on ESTR mutation rates in the germline and somatic tissue of their first-generation offspring.

Barber and co-authors demonstrated that transgenerational instability observed in the first-generation offspring of irradiated male mice is attributed to an elevated level of DNA damage, including single- and double-strand breaks (Barber et al., 2006). Given that the increased amount of DNA damage can facilitate tumor initiation and progression, the results of this study therefore provide a plausible explanation for the transgenerational effects of paternal irradiation on the risk of cancer among the offspring of irradiated parents. It should however be mentioned that to date there has been little experimental evidence for the transgenerational effects of paternal irradiation in humans. For example, Tawn and co-authors showed that the frequency of chromosome aberrations in the offspring of childhood cancer survivors of radiotherapy did not significantly differ from that in controls (Tawn et al., 2005). 
To establish whether paternal history of a low dietary methyl-donor intake could have resulted in transgenerational changes in ESTR mutation rates, treated males were mated to control females and ESTR mutation frequencies in the offspring of treated males conceived within 2 and 6 weeks after the end of dietary restriction were evaluated. Using the experimental approaches mentioned above, ESTR mutation frequencies were established in DNA samples extracted from brain and sperm of the offspring of treated and control males.

Brain and sperm tissues were analysed to establish whether transgenerational instability manifests in the $F_{1}$ germline and somatic tissue. This approach was taken from previous animal studies, which showed that transgenerational instability equally affected ESTR mutation rate in the germline and somatic tissues of $F_{1}$ offspring of male mice exposed to ionizing radiation and anticancer drugs (Barber et al., 2006; Barber et al., 2009; Hatch et al., 2007; Glen \& Dubrova, 2012; Abouzeid Ali et al., 2012).

Similarly to the data obtained on the directly treated males, the frequency of ESTR mutation in the $F_{1}$ offspring did not significantly differ from that in controls (Tables $\mathbf{3 . 6}$ and 3.7, Figure 3.8) in either somatic tissue or germline. These results therefore show that paternal methyl-donor deficiency does not destabilize the genomes of their first-generation offspring, thus implying the lack of transgenerational effects. 


\subsubsection{Effects of a methyl-donor deficient diet on ESTR mutation spectrum}

The forth and final objective of this project was to determine the impact of the methyl-donor deficient diet on the ESTR mutation spectrum. Several investigations have proposed that spontaneous ESTR mutation is attributed to replication slippage events (Yauk et al., 2002; Hardwick et al., 2009). A study conducted by Y.E. Dubrova in 2005 comparing the ESTR mutation spectrum of five strains of laboratory mice (including BALB/c) after an acute exposure to low-LET $\mathrm{X}$ or gamma rays at the pre-meiotic stages of spermatogenesis revealed that most mutation events involved the gain or loss of a relatively small number of repeat units, and the distributions of length changes were indistinguishable between the exposed and control males, with an overall significant bias towards gains of repeats (Dubrova, $2005 b)$. In this project, the spectra of ESTR mutation in DNA samples extracted from sperm and brain of control and treated animals, as well as their first generation offspring were evaluated and compared.

The percentage of gains and losses of repeats amongst the total of 130 unique ESTR mutations, recorded in the sperm tissue of control and test $F_{0}$ BALB/c males was first established. Similarly to previous studies (Dubrova, 2005b; Barber et al., 2009), the progenitor allele was assumed to be the non-mutant allele closest in size to the mutant allele (Figure 3.5). The data from 12, 18 and 22 week-old-mice were pooled together and the resulted frequency of gains and losses for the treated males did not significantly differ from that in controls (Table 3.8). Similarly, the frequency 
distributions of the germline length changes for the control and test groups in the two groups did not significantly differ (Figure 3.9).

The spectrum of ESTR mutation was also established for the 95 unique mutations found in sperm of the offspring of treated and control males. Similarly to the directly exposed animals, the incidence of gains and losses was indistinguishable between all $F_{1}$ control and test groups (Table 3.9). However, a comparison of the frequency distributions in the two groups revealed a significant difference between them, attributed to a shift toward large mutation events among the offspring of treated males (Figure 3.10). This phenomenon is more likely to be the cause of a statistical error/fluke but it could also be the attribution and propagation of a paternally induced transgenerational effect in the offspring of treated males, causing not the increase of mutations per se, but rather hinder a part of the replication procedure causing the accumulation of larger gains or losses for repeat units. Further work towards the elucidation of the yet unknown mechanism(s) of mutation induction in the ESTR loci will be able to illuminate the importance of this finding.

The spectra of ESTR mutation were also analysed for the 69 unique mutations found in the brain tissue of all offspring of control and test males. Again, the incidence of gains and losses was indistinguishable between all $F_{1}$ control and test groups (Table 3.10). Similarly, the frequency distributions of the $F_{1}$ of control and test groups did not significantly differ (Figure 3.11). Finally, $F_{1}$ data from both sperm and brain tissues were combined and the spectra of ESTR mutation for the offspring of control and test males were compared. The incidence of gains and losses in the two groups did not 
significantly differ (Table 3.11); the same was also true for the frequency distributions of ESTR mutations (Figure 3.12).

The results clearly indicate that the ESTR mutation spectrum between control and test groups in each tissue does not significantly differ. The data also revealed a high ESTR frequency in mutations regarding gains or losses of 2 repeat units. These findings support the theory of a replication slippage mechanism. Furthermore, the mutation spectrum of somatic ESTR mutations in control and test groups did not significantly differ (Figure 3.10) which indicates that a common mechanism may underline mutational process in the two tissues.

The results of this study therefore show that: (a) the spectra of ESTR mutations detected in DNA samples extracted from sperm of control and test males do not significantly differ; (b) the spectra of ESTR found in DNA samples extracted from sperm and brain of the first-generation offspring of control and test males do not substantially differ; (c) the majority of ESTR mutations belong to a class of small events involving the gain or loss of $\sim 2$ repeat units.

Considering the results of this study, it would appear that methyl-donor deficiency does not alter the spectra of ESTR mutation in the germline of directly treated male mice and their first-generation offspring.

\subsection{Phenotypic changes in somatic tissue $v$ germline effects}

In previous studies (Blount et al, 1997; Pogribny et al., 2006) the longterm effects of a folate deficiency were evaluated in somatic tissues of treated animals. Therefore their results, showing the transient effects of folate 
deficiency in mice, cannot be directly compared with the data obtained in this study, whose aim was to establish the long-term effects of folate deficiency in the germline of treated male mice. However, it is tempting to speculate that folate deficiency could potentially result in the long-term destabilization of the genome of somatic cells without affecting the germline of treated animals. It may appear that the efficiency of some mechanisms involved in the maintaining of genome stability in the male germline substantially exceeds that in somatic cells, which could provide better protection against a number of environmental insults, including prolonged low dietary folate deprivation. A genome-wide comparison of the epigenetic landscape of DNA samples taken from the germline and somatic tissues of treated animals may provide important insights into this issue. Dr Colin Glen is currently performing this work on DNA samples collected from animals analyzed in this study.

It should be noted that the results of a recent study by StarlardDavenport and co-authors provide support to the abovementioned notion. The authors compared the genome-wide pattern of DNA methylation of $\mathrm{CpG}$ islands and gene expression in liver samples taken from patients suffering from the non-alcoholic steatohepatitis (fatty liver) disease to those caused by a methyl deficient diet in mice (Starlard-Davenport et al., 2011). According to the results of this study, the expression of 164 genes was significantly altered in the liver of treated animals, and an inverse relationship was found between the level of gene expression and the pattern of $\mathrm{CpG}$ island methylation. Most importantly, differential methylation was observed for the genes involved with DNA damage repair processes, the lipid and glucose metabolism and liver remodelling (Starlard-Davenport et al., 2011). These findings therefore imply 
that the negative effects of a low dietary folate deprivation may be mostly pronounced in the liver, possibly affecting the genome stability of this tissue.

It is worth mentioning that the expression of the Ahcy gene in the liver of treated mice was found to be severely down-regulated (Starlard-Davenport et al., 2011). This gene encodes for the SAHH enzyme involved in the hydrolysis of SAH to homocysteine and adenosine (Bailey \& Gregory, 1999). A compromised expression of the Ahcy gene causes increase of SAH levels in the cell, which in its turn inhibits DNMT and therefore further increases the inability to methylate DNA caused by folate deprivation (Bailey \& Gregory 1999). This could be a plausible explanation on why the liver shows to be the tissue most affected by folate deficiency even though the determination of expression of the Ahcy gene in other tissues should be thoroughly investigated before it could be considered accountable for the damage to the liver.

\subsection{Pig-A assay}

Given that the analysis of ESTR mutation frequencies failed to detect any significant diet-related changes among the treated mice and their firstgeneration offspring, it therefore remains to be established whether the same is also true for other end-points. To this end, we initiated a pilot study aimed to analyse the mutagenic and transgenerational effects of methyl-deficient diet on the frequency of Pig-A mutations. Similarly to the ESTR study, the target tissue for the F0 mutagenic effects is actively proliferated bone marrow. The preliminary data fully confirm the results of the ESTR analysis showing no changes in the frequency of somatic mutation in the frequency of 
directly treated animals and their $F_{1}$ offspring, even though the sensitivity of the assay was compromised due to the very high variation between the control animals (Table 3.2.2.1).

\subsection{Conclusion}

To the best of my knowledge, this study represents the first systematic attempt to evaluate the effect of folate deficiency on mutation induction and transgenerational instability in mice. The results of my study suggest that 8weeks of low dietary methyl-donor intake does not affect ESTR mutation rate in the germline of treated male mice and does not destabilise the genomes of their first-generation offspring. This conclusion is based on the three sets of experimental data showing that: (i) ESTR mutation rate in the germline of treated animals sacrificed immediately after treatment or sampled 6 and 10 weeks after the end of dietary restriction does not significantly differ from that in age-matched control groups, (ii) the frequency of ESTR mutation in the germline and somatic tissueof first-generation offspring of treated males is similar to that in the offspring of control males, (iii) the spectra of ESTR mutation found in DNA samples taken from the treated males and their firstgeneration offspring do not significantly differ from those in controls.

The results of this study imply that mutagenicity of folate-deficient diet may not be sufficient to promote tumor development and therefore do not support the abovementioned hypothesis linking the alleged damaging effects of such a deficiency on DNA with the risk of cancer. The negative results of this work are in line with those of a study which failed to detect any measurable increases in the frequency of point mutations in colon epithelial 
cells collected from mice kept for 8 months on a folate-deficient diet (Linhart et al., 2009). It would therefore appear that some epigenetic alterations leading to global hypomethylation and causing major changes in the gene expression might be regarded as the key mechanism underlying dietaryrelated carcinogenesis (Voutounou et al., 2012)

\subsection{Future work}

The data from this thesis suggest that a dietary deficiency of methyl donors such as folate does not destabilise the genome of directly exposed male mice and their first-generation offspring. However, these results require further analysis. Firstly, the frequency of ESTR mutation in somatic tissues of treated animals should be established, thus providing further evidence for the effects of a methyl-donor deficient diet on mutation induction in mice. Secondly, the genome-wide level of DNA methylation in the germline and somatic tissues of treated animals should be investigated in detail. The methylated DNA immunoprecipitation (MeDIP) assay (Weber et al., 2005; Weber et al., 2007) coupled with methylation-specific PCR and bisulfate sequencing (Noonan et al., 1990; Herman et al., 1996) represents the most promising experimental approach for future work. In parallel, the global level of DNA methylation could also be established by reversed-phase highperformance liquid chromatography (Kuo et al., 1980) or the cytosine extension assay previously used by the Pogribny group (Pogribny et al., 2006).

Preliminary data obtained by $\mathrm{Dr}$ Colin Glen on the pattern of gene expression in the liver tissue of animals kept for 8 week on methyl-sufficient 
and methyl-deficient diet (Test $F_{0} A$ ) show that profound transcriptomic changes occurred over this period of dietary restriction (Figure 4.8.1). Using the NimbleGen $12 \times 135 \mathrm{~K}$ multiplex expression array, the patter of gene expressions of 42,575 transcripts was compared in the two groups, 2,086 of which showed at least two-fold changes in the exposed group at the False Discovery Rate at 0.05 . The majority of transcripts (80\%) were up-regulated in the treated group.

Figure 4.8.1. Pattern of gene expression in the Test $F_{0} A$ mice

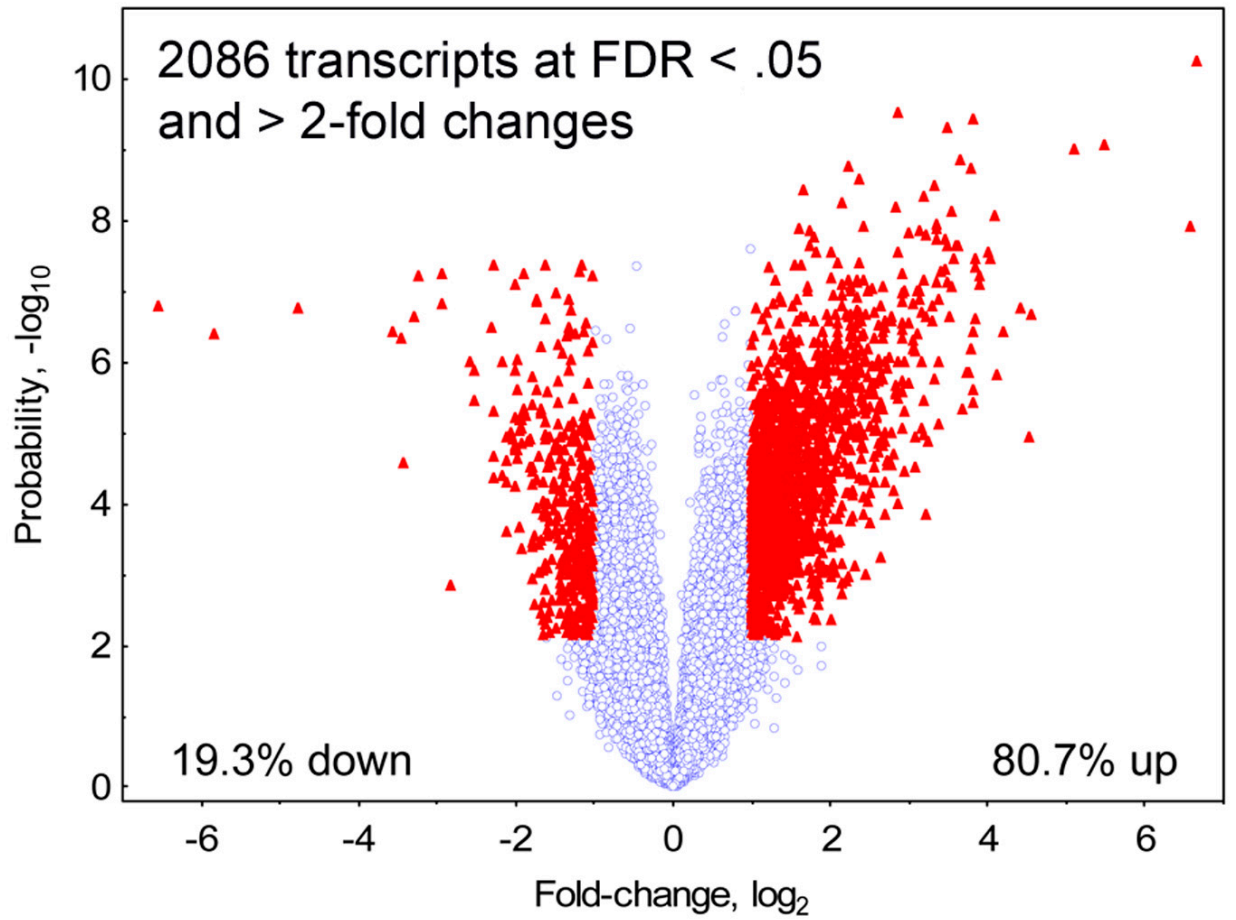

Finally, the pattern of gene expression following dietary restrictions should be analysed in detail using the microarray technique, coupled with the analysis of $\mathrm{CpG}$ island methylation, as previously performed in a pilot study by Starlard-Davenport and co-authors (Starlard-Davenport et al., 2011). 


\section{Appendix}

\subsection{Methyl donor diets}

\subsubsection{Methyl sufficient diet}

Dyets inc. Diet \#D518894. Choline sufficient and iron supplemented L-AA defied diet with $0.18 \%$ methionine and $1 \%$ succinyl sulfathiazole.

Ingredients

L-Alanine

L-Aspartic Acid

L-Cystine

L-Glutamic Acid

Glycine

L-Histidine

L-Isoleucine

L-Leucine

L-Lysine $\mathrm{HCl}$

L-Methionine

L-Phenylalanine

L-Proline

L-Serine

L-Threonine

L-Tryptophan

L-Tyrosine

L-Valine

Total L-Amino Acid g/kg

5.10

15.80

3.70

28.90

6.20

3.40

6.10

10.50

9.10

1.80

7.30

7.60

7.20

4.60

1.80

5.70

6.30

143.80 
Cornstarch

100.00

Sucrose

396.57

Cellulose (microcrystalline)

50.00

Corn Oil

50.00

Primex

100.00

Salt Mix \#215001 (no Fe added)

35.00

Sodium Bicarbonate

4.30

Vitamin Mix \#300050

10.00

Choline Bitartrate

14.4

Ferric Citrate, U.S.P.

0.33

Succinyl Sulfathiazole

10 


\subsubsection{Methyl deficient diet}

Dyets inc. Diet \#D518892. Choline deficient and iron supplemented L-AA defied diet with $0.18 \%$ methionine, $0 \%$ folic acid and $1 \%$ succinyl sulfathiazole.

Ingredients

L-Alanine

L-Aspartic Acid

L-Cystine

L-Glutamic Acid

Glycine

L-Histidine

L-Isoleucine

L-Leucine

L-Lysine $\mathrm{HCl}$

L-Methionine

L-Phenylalanine

L-Proline

L-Serine

L-Threonine

L-Tryptophan

L-Tyrosine

L-Valine

Total L-Amino Acid g/kg

5.10

15.80

3.70

28.90

6.20

3.40

6.10

10.50

9.10

1.80

7.30

7.60

7.20

4.60

1.80

5.70

6.30

143.80 
Cornstarch

100.00

Sucrose

396.57

Cellulose (microcrystalline)

50.00

Corn Oil

50.00

Primex

100.00

Salt Mix \#215001 (no Fe added)

35.00

Sodium Bicarbonate

4.30

Vitamin Mix \#317759 (Folate Free)

10.00

Choline Bitartrate

0.00

Ferric Citrate, U.S.P.

0.33

Succinyl Sulfathiazole

10 


\subsection{Summary of ESTR data}

\subsubsection{All ESTR mutation data: $F_{0}$ sperm}

\begin{tabular}{|c|c|c|c|c|c|}
\hline Tissue & Group & Animal & Mutant & $\begin{array}{c}\text { Difference } \\
(\mathrm{mm})\end{array}$ & Comment \\
\hline \multirow[t]{5}{*}{ Sperm } & Control A & S.3 & A9 & -4 & \\
\hline & & & A4 & $\overline{9}$ & \\
\hline & & & C4 & -2 & Mosaic \\
\hline & & & B5 & 3 & \\
\hline & & & $\mathrm{C} 2$ & 2 & \\
\hline \multirow[t]{7}{*}{ Sperm } & Control A & 5.4 & D12 & 7 & \\
\hline & & & E9 & 8 & \\
\hline & & & D4 & 2 & \\
\hline & & & C3 & -3 & \\
\hline & & & B12 & -3 & \\
\hline & & & C8 & -2 & \\
\hline & & & G3 & -2 & \\
\hline \multirow[t]{4}{*}{ Sperm } & Control A & S.5 & G12 & 7 & \\
\hline & & & F9 & -7 & Mosaic \\
\hline & & & E8 & -2 & \\
\hline & & & E1 & -2 & \\
\hline \multirow[t]{4}{*}{ Sperm } & Control A & S.6 & E7 & 3 & \\
\hline & & & A11 & 2 & \\
\hline & & & E5 & 3 & \\
\hline & & & D3 & -4 & \\
\hline
\end{tabular}




\begin{tabular}{|c|c|c|c|c|c|}
\hline Tissue & Group & Animal & Mutant & $\begin{array}{c}\text { Difference } \\
(\mathrm{mm})\end{array}$ & Comment \\
\hline \multirow[t]{5}{*}{ Sperm } & Test A & D.3 & A12 & 4 & \\
\hline & & & B9 & 4 & \\
\hline & & & B11 & 4 & \\
\hline & & & C6 & 3 & \\
\hline & & & E8 & 2 & \\
\hline \multirow[t]{5}{*}{ Sperm } & Test A & D.4 & $\mathrm{C} 2$ & -4 & \\
\hline & & & F10 & 2 & \\
\hline & & & $\mathrm{H} 7$ & 4 & \\
\hline & & & A7 & 5 & \\
\hline & & & D5 & -2 & \\
\hline \multirow[t]{6}{*}{ Sperm } & Test A & D.5 & B9 & -2 & \\
\hline & & & $\mathrm{C7}$ & -3 & \\
\hline & & & G7 & -4 & \\
\hline & & & $\mathrm{H} 2$ & -4 & \\
\hline & & & C11 & -3 & \\
\hline & & & E3 & 4 & \\
\hline \multirow[t]{5}{*}{ Sperm } & Test A & D.3 & A12 & 4 & \\
\hline & & & B9 & 4 & \\
\hline & & & B11 & 4 & \\
\hline & & & C6 & 3 & \\
\hline & & & E8 & 2 & \\
\hline \multirow[t]{5}{*}{ Sperm } & Test A & D.4 & $\mathrm{C} 2$ & -4 & \\
\hline & & & F10 & 2 & \\
\hline & & & $\mathrm{H} 7$ & 4 & \\
\hline & & & A7 & 5 & \\
\hline & & & D5 & -2 & \\
\hline \multirow[t]{2}{*}{ Sperm } & Test A & D.5 & B9 & -2 & \\
\hline & & & $\mathrm{C7}$ & -3 & \\
\hline
\end{tabular}




\begin{tabular}{|c|c|c|c|c|}
\hline & & & G7 & -4 \\
\hline & & & $\mathrm{H} 2$ & -4 \\
\hline & & & C11 & -3 \\
\hline & & & E3 & 4 \\
\hline Sperm & Test A & D.3 & A12 & 4 \\
\hline & & & B9 & 4 \\
\hline & & & B11 & 4 \\
\hline & & & C6 & 3 \\
\hline & & & E8 & 2 \\
\hline Sperm & Test A & D.4 & $\mathrm{C} 2$ & -4 \\
\hline & & & F10 & 2 \\
\hline & & & $\mathrm{H} 7$ & 4 \\
\hline & & & A7 & 5 \\
\hline & & & D5 & -2 \\
\hline Sperm & Test $\mathrm{A}$ & D.5 & B9 & -2 \\
\hline & & & C7 & -3 \\
\hline & & & G7 & -4 \\
\hline & & & $\mathrm{H} 2$ & -4 \\
\hline & & & C11 & -3 \\
\hline & & & E3 & 4 \\
\hline
\end{tabular}




\begin{tabular}{|c|c|c|c|c|c|}
\hline Tissue & Group & Animal & Mutant & $\begin{array}{l}\text { Difference } \\
(\mathrm{mm})\end{array}$ & Comment \\
\hline \multirow[t]{5}{*}{ Sperm } & Control B & S.11 & A1 & 3 & \\
\hline & & & B11 & -2 & \\
\hline & & & $\mathrm{H} 8$ & -3 & \\
\hline & & & E6 & 4 & \\
\hline & & & A12 & -2 & \\
\hline \multirow[t]{2}{*}{ Sperm } & Control B & S.12 & C7 & -2 & \\
\hline & & & A12 & -3 & \\
\hline \multirow[t]{7}{*}{ Sperm } & Control B & 5.13 & E7 & 4 & \\
\hline & & & C9 & $\overline{9}$ & \\
\hline & & & G7 & 3 & \\
\hline & & & $\mathrm{H} 6$ & -7 & \\
\hline & & & D11 & 5 & \\
\hline & & & C7 & 3 & \\
\hline & & & B5 & 3 & \\
\hline \multirow[t]{2}{*}{ Sperm } & Control B & S.14 & F5 & 3 & \\
\hline & & & D1 & 3 & \\
\hline & & & & & \\
\hline
\end{tabular}




\begin{tabular}{|c|c|c|c|c|c|}
\hline Tissue & Group & Animal & Mutant & $\begin{array}{c}\text { Difference } \\
\text { (mm) }\end{array}$ & Comment \\
\hline \multirow[t]{7}{*}{ Sperm } & Test B & D.11 & G10 & -3 & \\
\hline & & & E10 & -2 & \\
\hline & & & A2 & -9 & \\
\hline & & & A7 & -2 & \\
\hline & & & A3 & 3 & \\
\hline & & & F2 & -2 & \\
\hline & & & F1 & -2 & \\
\hline \multirow[t]{5}{*}{ Sperm } & Test B & D.12 & B10 & -3 & \\
\hline & & & A3 & -2 & \\
\hline & & & A7 & -2 & \\
\hline & & & F6 & 2 & \\
\hline & & & A11 & -4 & \\
\hline \multirow[t]{6}{*}{ Sperm } & Test B & D.13 & F12 & -2 & \\
\hline & & & G9 & -3 & \\
\hline & & & G5 & 3 & \\
\hline & & & A9 & -4 & \\
\hline & & & F6 & -7 & \\
\hline & & & E4 & -8 & \\
\hline \multirow[t]{2}{*}{ Sperm } & Test B & D.14 & $\mathrm{H} 12$ & -2 & \\
\hline & & & G1 & -2 & \\
\hline
\end{tabular}




\begin{tabular}{|c|c|c|c|c|c|}
\hline Tissue & Group & Animal & Mutant & $\begin{array}{l}\text { Difference } \\
\text { (mm) }\end{array}$ & Comment \\
\hline \multirow{6}{*}{ Sperm } & Control C & S.16 & B11 & 2 & \\
\hline & & & A8 & -2 & \\
\hline & & & F2 & 5 & \\
\hline & & & B9 & 3 & \\
\hline & & & F7 & -3 & \\
\hline & & & $\mathrm{H} 2$ & 5 & \\
\hline \multirow[t]{7}{*}{ Sperm } & Control C & S.17 & G11 & 3 & \\
\hline & & & B10 & 4 & \\
\hline & & & $\mathrm{D} 6$ & 2 & \\
\hline & & & $\mathrm{C} 1$ & 40 & \\
\hline & & & B8 & 4 & \\
\hline & & & $\mathrm{E} 4$ & 2 & \\
\hline & & & A2 & 4 & \\
\hline \multirow[t]{7}{*}{ Sperm } & Control C & S.18 & A11 & 7 & \\
\hline & & & B10 & 5 & \\
\hline & & & B3 & -2 & \\
\hline & & & $\mathrm{E} 1$ & 4 & Mosaic \\
\hline & & & F8 & 5 & \\
\hline & & & $\mathrm{H} 6$ & 2 & \\
\hline & & & D4 & 4 & \\
\hline \multirow[t]{8}{*}{ Sperm } & Control C & S.19 & G10 & -5 & \\
\hline & & & D9 & -5 & \\
\hline & & & F5 & -8 & \\
\hline & & & $\overline{\mathrm{D} 2}$ & -2 & \\
\hline & & & A6 & 8 & Mosaic \\
\hline & & & $\mathrm{H} 11$ & 21 & \\
\hline & & & A9 & -2 & \\
\hline & & & & & \\
\hline
\end{tabular}




\begin{tabular}{|c|c|c|c|c|c|}
\hline Tissue & Group & Animal & Mutant & $\begin{array}{c}\text { Difference } \\
(\mathrm{mm})\end{array}$ & Comment \\
\hline \multirow[t]{7}{*}{ Sperm } & Test C & D.16 & $\mathrm{C} 2$ & 3 & \\
\hline & & & $\mathrm{H} 1$ & 3 & \\
\hline & & & A9 & 3 & \\
\hline & & & A6 & -3 & \\
\hline & & & E9 & -2 & \\
\hline & & & $\mathrm{C7}$ & -7 & \\
\hline & & & F4 & 2 & \\
\hline \multirow[t]{5}{*}{ Sperm } & Test C & D.17 & $\mathrm{H} 7$ & 6 & \\
\hline & & & C4 & 4 & \\
\hline & & & A3 & 5 & \\
\hline & & & E3 & 8 & \\
\hline & & & F1 & -4 & \\
\hline \multirow{8}{*}{ Sperm } & Test C & D.18 & D4 & 9 & \\
\hline & & & D11 & -2 & \\
\hline & & & G9 & -3 & \\
\hline & & & D3 & 2 & \\
\hline & & & F1 & 2 & \\
\hline & & & G7 & -3 & \\
\hline & & & C5 & -3 & \\
\hline & & & B2 & 3 & \\
\hline \multirow[t]{6}{*}{ Sperm } & Test C & D.19 & H3 & 3 & \\
\hline & & & G2 & 3 & \\
\hline & & & F8 & -3 & \\
\hline & & & A6 & -2 & \\
\hline & & & A2 & -4 & \\
\hline & & & F10 & 2 & \\
\hline
\end{tabular}


5.2.2. All ESTR mutation data: $F_{1}$ sperm

\begin{tabular}{|c|c|c|c|c|c|}
\hline Tissue & Group & Animal & Mutant & $\begin{array}{c}\text { Difference } \\
(\mathrm{mm})\end{array}$ & Comment \\
\hline \multirow[t]{4}{*}{ Sperm } & Control F1B & S.13.b1 & A4 & -2 & \\
\hline & & & A1 & 3 & \\
\hline & & & D4 & 3 & \\
\hline & & & E4 & -3 & \\
\hline \multirow[t]{2}{*}{ Sperm } & Control F1B & S.14.b1 & F6 & -3 & \\
\hline & & & $\mathrm{D} 2$ & -2 & \\
\hline \multirow[t]{6}{*}{ Sperm } & Control F1B & S.15.a2 & A10 & -4 & \\
\hline & & & A5 & -4 & \\
\hline & & & C11 & -2 & \\
\hline & & & B9 & -3 & \\
\hline & & & A7 & 2 & \\
\hline & & & A6 & -3 & \\
\hline \multirow[t]{8}{*}{ Sperm } & Control F1B & S.15.a3 & D9 & 4 & \\
\hline & & & B1 & -3 & \\
\hline & & & G1 & 8 & \\
\hline & & & B11 & 4 & \\
\hline & & & D12 & -2 & \\
\hline & & & C3 & -3 & \\
\hline & & & E2 & -4 & \\
\hline & & & & & \\
\hline
\end{tabular}




\begin{tabular}{|c|c|c|c|c|c|}
\hline Tissue & Group & Animal & Mutant & $\begin{array}{l}\text { Difference } \\
(\mathrm{mm})\end{array}$ & Comment \\
\hline \multirow[t]{5}{*}{ Sperm } & Test F1B & D.14.b1 & A11 & (i.m) & \\
\hline & & & D11 & 3 & \\
\hline & & & G3 & 2 & \\
\hline & & & E11 & 4 & \\
\hline & & & G4 & -4 & \\
\hline \multirow[t]{10}{*}{ Sperm } & Test F1B & D.14.b2 & C12 & 14 & \\
\hline & & & E10 & 6 & \\
\hline & & & C8 & 9 & \\
\hline & & & H8 & 5 & \\
\hline & & & B7 & 6 & \\
\hline & & & E4 & 5 & \\
\hline & & & $\mathrm{H} 4$ & 8 & \\
\hline & & & F7 & 6 & \\
\hline & & & F4 & 3 & \\
\hline & & & $\mathrm{H} 5$ & 2 & \\
\hline \multirow[t]{4}{*}{ Sperm } & Test F1B & D.15.b1 & D9 & 6 & \\
\hline & & & C4 & 4 & \\
\hline & & & E9 & 4 & \\
\hline & & & E4 & -3 & \\
\hline \multirow[t]{3}{*}{ Sperm } & Test F1B & D.15.b2 & D9 & -3 & \\
\hline & & & G3 & -4 & \\
\hline & & & A7 & 7 & \\
\hline
\end{tabular}




\begin{tabular}{|c|c|c|c|c|c|}
\hline Tissue & Group & Animal & Mutant & $\begin{array}{c}\text { Difference } \\
(\mathrm{mm})\end{array}$ & Comment \\
\hline \multirow[t]{8}{*}{ Sperm } & Control F1C & S.16.b1 & B11 & -2 & \\
\hline & & & G7 & 2 & \\
\hline & & & B10 & 2 & \\
\hline & & & B8 & -2 & \\
\hline & & & B3 & 2 & \\
\hline & & & B1 & -2 & \\
\hline & & & E4 & 2 & \\
\hline & & & $\mathrm{H} 2$ & 2 & \\
\hline \multirow[t]{5}{*}{ Sperm } & Control F1C & S.17.a1 & A11 & -2 & \\
\hline & & & A10 & -3 & \\
\hline & & & A7 & -2 & \\
\hline & & & $\mathrm{F} 4$ & -2 & \\
\hline & & & G5 & -2 & \\
\hline \multirow[t]{7}{*}{ Sperm } & Control F1C & S.18.a1 & A9 & 2 & \\
\hline & & & A3 & -2 & \\
\hline & & & B12 & 6 & \\
\hline & & & A8 & 2 & \\
\hline & & & G5 & 3 & \\
\hline & & & F5 & 2 & \\
\hline & & & F5 & -2 & \\
\hline
\end{tabular}




\begin{tabular}{|c|c|c|c|c|c|}
\hline Tissue & Group & Animal & Mutant & $\begin{array}{c}\text { Difference } \\
(\mathrm{mm})\end{array}$ & Comment \\
\hline \multirow[t]{10}{*}{ Sperm } & Test F1C & D.17.b1 & D6 & 2 & \\
\hline & & & D4 & 4 & \\
\hline & & & $\mathrm{H} 4$ & -2 & \\
\hline & & & D9 & -4 & Mosaic \\
\hline & & & C3 & 2 & \\
\hline & & & A11 & -2 & \\
\hline & & & D11 & 2 & \\
\hline & & & $\mathrm{H} 8$ & 2 & \\
\hline & & & B5 & 2 & \\
\hline & & & $\mathrm{H} 2$ & -4 & \\
\hline \multirow[t]{7}{*}{ Sperm } & Test F1C & D.17.b2 & C10 & 3 & \\
\hline & & & D9 & -2 & \\
\hline & & & C8 & 3 & \\
\hline & & & $\mathrm{H} 7$ & 2 & \\
\hline & & & A11 & -2 & \\
\hline & & & F7 & 3 & \\
\hline & & & A6 & 2 & \\
\hline \multirow[t]{6}{*}{ Sperm } & Test F1C & D.18.a1 & C10 & -2 & \\
\hline & & & E10 & -3 & \\
\hline & & & G10 & 2 & \\
\hline & & & $\mathrm{C} 4$ & -2 & \\
\hline & & & F11 & 2 & \\
\hline & & & D5 & -3 & \\
\hline \multirow[t]{6}{*}{ Sperm } & Test F1C & D.18.a2 & E11 & 50 & \\
\hline & & & $\mathrm{H} 9$ & -2 & \\
\hline & & & C6 & -2 & \\
\hline & & & E12 & -2 & \\
\hline & & & A10 & -2 & \\
\hline & & & A2 & -2 & \\
\hline
\end{tabular}


5.2.3. All ESTR mutation data: $F_{1}$ brain

\begin{tabular}{|c|c|c|c|c|c|}
\hline Tissue & Group & Animal & Mutant & $\begin{array}{c}\text { Difference } \\
(\mathrm{mm})\end{array}$ & Comment \\
\hline \multirow[t]{5}{*}{ Brain } & Control F1B & S.13.b1 & $\mathrm{H} 2$ & 2 & \\
\hline & & & C8 & 5 & \\
\hline & & & $\mathrm{H} 2$ & 4 & \\
\hline & & & G6 & 5 & \\
\hline & & & $\mathrm{D} 1$ & -2 & \\
\hline \multirow[t]{6}{*}{ Brain } & Control F1B & S.14.b1 & G7 & 3 & \\
\hline & & & C6 & 2 & \\
\hline & & & A7 & 2 & \\
\hline & & & B11 & 2 & \\
\hline & & & $\mathrm{H} 7$ & -2 & \\
\hline & & & E4 & -5 & \\
\hline \multirow[t]{2}{*}{ Brain } & Control F1B & S.15.a2 & A11 & -4 & Mosaic \\
\hline & & & D2 & -2 & \\
\hline \multirow[t]{4}{*}{ Brain } & Control F1B & S.15.a3 & B11 & -4 & \\
\hline & & & C10 & 7 & \\
\hline & & & C6 & 2 & \\
\hline & & & F7 & -2 & \\
\hline
\end{tabular}




\begin{tabular}{|c|c|c|c|c|c|}
\hline Tissue & Group & Animal & Mutant & $\begin{array}{c}\text { Difference } \\
(\mathrm{mm})\end{array}$ & Comment \\
\hline \multirow[t]{5}{*}{ Brain } & Test F1B & D.14.b1 & G12 & 4 & \\
\hline & & & $\mathrm{F} 5$ & -2 & \\
\hline & & & $\mathrm{C} 6$ & 2 & \\
\hline & & & B3 & -4 & \\
\hline & & & B8 & -2 & \\
\hline \multirow[t]{3}{*}{ Brain } & Test F1B & D.14.b2 & A9 & -2 & \\
\hline & & & C11 & 2 & \\
\hline & & & G8 & 2 & \\
\hline \multirow[t]{5}{*}{ Brain } & Test F1B & D.15.b1 & D11 & 6 & \\
\hline & & & $\mathrm{F} 7$ & -2 & \\
\hline & & & F3 & 6 & \\
\hline & & & $\mathrm{F} 1$ & 5 & \\
\hline & & & F1 & 6 & \\
\hline \multirow[t]{4}{*}{ brain } & Test F1B & D.15.b2 & F9 & -3 & \\
\hline & & & E10 & 6 & \\
\hline & & & G5 & 2 & \\
\hline & & & $\mathrm{E} 4$ & 2 & \\
\hline
\end{tabular}




\begin{tabular}{|c|c|c|c|c|c|}
\hline Tissue & Group & Animal & Mutant & $\begin{array}{c}\text { Difference } \\
(\mathrm{mm})\end{array}$ & Comment \\
\hline \multirow[t]{5}{*}{ Brain } & ConF1C & S.16.b1 & $\mathrm{C4}$ & 4 & \\
\hline & & & G5 & -4 & \\
\hline & & & B4 & 3 & \\
\hline & & & E4 & 2 & \\
\hline & & & C6 & 3 & \\
\hline \multirow[t]{8}{*}{ Brain } & ConF1C & S.17.a1 & $\mathrm{H} 8$ & -2 & \\
\hline & & & E2 & 2 & \\
\hline & & & F11 & 8 & \\
\hline & & & D10 & -5 & \\
\hline & & & G10 & -3 & \\
\hline & & & E9 & 2 & \\
\hline & & & A8 & 7 & \\
\hline & & & E7 & -4 & \\
\hline \multirow[t]{4}{*}{ Brain } & ConF1C & S.18.a1 & C6 & 3 & \\
\hline & & & $\mathrm{H} 10$ & 10 & \\
\hline & & & G6 & -3 & \\
\hline & & & E3 & -2 & \\
\hline \multirow[t]{2}{*}{ Brain } & ConF1C & S.19.a1 & F11 & -3 & \\
\hline & & & G2 & 4 & \\
\hline
\end{tabular}




\begin{tabular}{|c|c|c|c|c|c|}
\hline Tissue & Group & Animal & Mutant & $\begin{array}{c}\text { Difference } \\
(\mathrm{mm})\end{array}$ & Comment \\
\hline \multirow[t]{5}{*}{ Brain } & Test F1C & D.17.b1 & D1 & 4 & \\
\hline & & & $\mathrm{A} 2$ & 2 & \\
\hline & & & $\mathrm{H} 7$ & -2 & \\
\hline & & & C9 & 2 & \\
\hline & & & E4 & 3 & \\
\hline \multirow[t]{2}{*}{ Brain } & Test F1C & D.17.b2 & A1 & 4 & \\
\hline & & & F5 & 4 & \\
\hline \multirow[t]{5}{*}{ Brain } & Test F1C & D.18.a1 & $\mathrm{H} 10$ & 2 & \\
\hline & & & F2 & -2 & \\
\hline & & & D12 & 2 & \\
\hline & & & B7 & -2 & \\
\hline & & & F3 & 2 & \\
\hline \multirow[t]{4}{*}{ Brain } & Test F1C & D.18.a2 & A9 & -4 & \\
\hline & & & C9 & -3 & \\
\hline & & & F3 & -2 & \\
\hline & & & B1 & 2 & \\
\hline
\end{tabular}




\subsection{Summary of Pig-A data}

5.3.1. All ENU, Control and Treated $F_{0}$ data

\begin{tabular}{|l|c|c|c|}
\hline $\begin{array}{l}\text { GROUPS } \\
\text { All Fo }\end{array}$ & DATE & NAME & $\begin{array}{c}\text { No } \\
\text { events }\end{array}$ \\
\hline
\end{tabular}

\begin{tabular}{|l|l|r|r|r|}
\hline ENU & Gontrol & $14 / 06 / 2010$ & CON1 & 17 \\
\hline & 8 weeks & $14 / 06 / 2010$ & CON2 & 32 \\
\hline & & $14 / 06 / 2010$ & CON3 & 77 \\
\hline & ENU Group & $14 / 06 / 2010$ & ENU1 & 828 \\
\hline & 8 weeks & $14 / 06 / 2010$ & ENU2 & 229 \\
\hline & & $14 / 06 / 2010$ & ENU3 & 213 \\
\hline
\end{tabular}

\begin{tabular}{|l|l|r|r|r|}
\hline Group A & Group & $27 / 05 / 2010$ & SUF6 & 1 \\
\hline & 12 weeks & $27 / 05 / 2010$ & SUF7 & 1 \\
\hline & & $27 / 05 / 2010$ & SUF8 & 4 \\
\hline & Deficient & & & 5 \\
\hline & Group & $27 / 05 / 2010$ & DEF6 & 9 \\
\hline & 12 weeks & $27 / 05 / 2010$ & DEF7 & 11 \\
\hline
\end{tabular}

\begin{tabular}{|l|l|l|l|r|}
\hline Group B & Control & $06 / 07 / 2010$ & SUF11 & 3 \\
\hline
\end{tabular}




\begin{tabular}{|l|l|r|r|r|}
\hline & Group & & & \\
\hline & 14 weeks & $06 / 07 / 2010$ & SUF12 & 16 \\
\hline & & $07 / 07 / 2010$ & SUF13 & 17 \\
\hline & & $07 / 07 / 2010$ & SUF14 & 156 \\
\hline & & $07 / 07 / 2010$ & SUF15 & 15 \\
\hline & & & & \\
\hline & Geficient & & & \\
\hline & Group & $06 / 07 / 2010$ & DEF11 & 25 \\
\hline & 14 weeks & $06 / 07 / 2010$ & DEF12 & 32 \\
\hline & & $06 / 07 / 2010$ & DEF13 & 15 \\
\hline & & $07 / 07 / 2010$ & DEF14 & 5 \\
\hline & & $07 / 07 / 2010$ & DEF15 & 13 \\
\hline
\end{tabular}

\begin{tabular}{|l|l|r|r|r|}
\hline Group C & Group & $17 / 08 / 2010$ & SUF16 & 9 \\
\hline & 20 weeks & $17 / 08 / 2010$ & SUF17 & 15 \\
\hline & & $17 / 08 / 2010$ & SUF18 & 5 \\
\hline & & $18 / 08 / 2010$ & SUF19 & 19 \\
\hline & & $18 / 08 / 2010$ & SUF20 & 6 \\
\hline Group C & & $17 / 08 / 2010$ & DEF16 & 15 \\
\hline & & $17 / 08 / 2010$ & DEF17 & 1,229 \\
\hline & & $18 / 08 / 2010$ & DEF18 & 7 \\
\hline & & $18 / 08 / 2010$ & DEF19 & 29 \\
\hline & & $18 / 08 / 2010$ & DEF20 & 96 \\
\hline
\end{tabular}




\subsubsection{All Control and Treated $F_{1}$ data}

\begin{tabular}{|l|c|c|c|}
\hline GROUPS & DATE & NAME & No \\
All F1 & & & events \\
\hline
\end{tabular}

\begin{tabular}{|c|c|c|c|c|}
\hline $\begin{array}{l}\text { Group } \\
\text { B.F1 }\end{array}$ & $\begin{array}{l}\text { Control } \\
\text { Group }\end{array}$ & $24 / 08 / 2010$ & SUF11.a.1 & 35 \\
\hline & 8 weeks & $24 / 08 / 2010$ & SUF12.b.1 & 18 \\
\hline & & $24 / 08 / 2010$ & SUF13.b.1 & 27 \\
\hline & & $25 / 08 / 2010$ & SUF14.b.1 & 55 \\
\hline & & $25 / 08 / 2010$ & SUF15.a.1 & 1,773 \\
\hline & & $25 / 08 / 2010$ & SUF15.a.3 & 43 \\
\hline \multirow[t]{6}{*}{$\begin{array}{l}\text { Group } \\
\text { B.F1 }\end{array}$} & $\begin{array}{l}\text { Deficient } \\
\text { Group }\end{array}$ & $24 / 08 / 2010$ & DEF12.a.1 & 42 \\
\hline & 8 weeks & $24 / 08 / 2010$ & DEF13.b.1 & 22 \\
\hline & & $24 / 08 / 2010$ & DEF14.a.1 & 54 \\
\hline & & $25 / 08 / 2010$ & DEF14.b.1 & 30 \\
\hline & & $25 / 08 / 2010$ & DEF14.b.2 & 12 \\
\hline & & $25 / 08 / 2010$ & DEF15.b.2 & 146 \\
\hline
\end{tabular}

\begin{tabular}{|c|c|c|c|c|}
\hline \multirow[t]{4}{*}{$\begin{array}{l}\text { Group } \\
\text { C.F1 }\end{array}$} & $\begin{array}{l}\text { Control } \\
\text { Group }\end{array}$ & 28/09/2010 & SUF16.b.1 & 42 \\
\hline & 8 weeks & $28 / 09 / 2010$ & SUF17.a.1 & 12 \\
\hline & & $28 / 09 / 2010$ & SUF17.b.1 & 22 \\
\hline & & $29 / 09 / 2010$ & SUF18.a.1 & 21 \\
\hline
\end{tabular}




\begin{tabular}{|l|l|r|r|r|}
\hline & & $29 / 09 / 2010$ & SUF19.a.1 & 14 \\
\hline & & $29 / 09 / 2010$ & SUF19.b.1 & 18 \\
\hline $\begin{array}{l}\text { Group } \\
\text { C.F1 }\end{array}$ & $\begin{array}{l}\text { Deficient } \\
\text { Group }\end{array}$ & $28 / 09 / 2010$ & DEF17.b.1 & 4 \\
\hline & 8 weeks & $28 / 09 / 2010$ & DEF18.a.1 & 22 \\
\hline & & $28 / 09 / 2010$ & DEF18.a.2 & 3 \\
\hline & & $29 / 09 / 2010$ & DEF20.a.1 & 118 \\
\hline & & $29 / 09 / 2010$ & DEF20.a.2 & 3,313 \\
\hline & & $29 / 09 / 2010$ & DEF20.a.3 & 5 \\
\hline
\end{tabular}

5.3.3. All $F_{0}$ and $F_{1}$ combined data

\begin{tabular}{|l|c|c|c|}
\hline $\begin{array}{l}\text { GROUPS } \\
\text { All Controls }\end{array}$ & AGE & NAME & $\begin{array}{c}\text { No } \\
\text { events }\end{array}$ \\
\hline
\end{tabular}

\begin{tabular}{|l|r|r|r|r|}
\hline F0 & & 8 weeks & CON1 & 17 \\
\hline & & 8 weeks & CON2 & 32 \\
\hline & & 8 weeks & CON3 & 77 \\
\hline
\end{tabular}

\begin{tabular}{|l|r|r|r|r|}
\hline Group A & & 12 weeks & SUF6 & 1 \\
\hline & & 12 weeks & SUF7 & 1 \\
\hline & & 12 weeks & SUF8 & 4 \\
\hline
\end{tabular}

\begin{tabular}{|l|r|r|r|r|}
\hline Group B & 14 weeks & SUF11 & 3 \\
\hline & & 14 weeks & SUF12 & 16 \\
\hline
\end{tabular}




\begin{tabular}{|r|r|r|r|r|}
\hline & 14 weeks & SUF13 & 17 \\
\hline & & 14 weeks & SUF14 & 156 \\
\hline & & 14 weeks & SUF15 & 15 \\
\hline
\end{tabular}

\begin{tabular}{|l|r|r|r|r|}
\hline Group C & 20 weeks & SUF16 & 9 \\
\hline & & 20 weeks & SUF17 & 15 \\
\hline & & 20 weeks & SUF18 & 5 \\
\hline & & 20 weeks & SUF19 & 19 \\
\hline & & 20 weeks & SUF20 & 6 \\
\hline
\end{tabular}

\begin{tabular}{|c|c|c|c|}
\hline \multirow[t]{6}{*}{ Group B.F1 } & 8 weeks & SUF11.a.1 & 35 \\
\hline & 8 weeks & SUF12.b.1 & 18 \\
\hline & 8 weeks & SUF13.b.1 & 27 \\
\hline & 8 weeks & SUF14.b.1 & 55 \\
\hline & 8 weeks & SUF15.a.1 & 1,773 \\
\hline & 8 weeks & SUF15.a.3 & 43 \\
\hline
\end{tabular}

\begin{tabular}{|l|r|r|r|r|}
\hline Group C.F1 & & 8 weeks & SUF16.b.1 & 42 \\
\hline & & 8 weeks & SUF17.a.1 & 12 \\
\hline & & 8 weeks & SUF17.b.1 & 22 \\
\hline & & 8 weeks & SUF18.a.1 & 21 \\
\hline & & 8 weeks & SUF19.a.1 & 14 \\
\hline & & 8 weeks & SUF19.b.1 & 18 \\
\hline
\end{tabular}




\section{References:}

Abouzeid Ali H.E., Barber R.C., Dubrova Y.E. (2012). The effects of maternal irradiation during adulthood on mutation induction and transgenerational instability in mice. Mutation Research, 732, 21-25.

Alberg A.J., Selhub J., Shah K.V., Viscidi R.P., Comstock G.W., Helzlsouer K.J. (2000). The risk of cervical cancer in relation to serum concentrations of folate, vitamin $\mathrm{B}_{12}$, and homocysteine. Cancer Epidemiology Biomarkers \& Prevention, 9 (7), 761-764.

Amaral C.L., Bueno B., Burim R.V., Queiroz R.H., Bianchi L., Antunes L.M. (2011). The effects of dietary supplementation of methionine on genomic stability and p53 gene promoter methylation in rats. Mutation Research, Genetic Toxicology and Environmental Mutagenesis, 722 (1), 78-83.

Ames B.N. (2001). DNA damage from micronutrient deficiencies is likely to be a major cause of cancer. Mutation Research, 475, 7-20.

Ames B.N., Wakimoto P. (2002). Are vitamin and mineral deficiencies a major cancer risk? Nature Reviews Cancer, 2, 694-704.

An Q., Robins P., Lindahl T., Barnes D.E. (2005). C->T mutagenesis and gamma-radiation sensitivity due to deficiency in the Smug1 and Ung DNA glycosylases. EMBO Journal, 24, 2205-2213.

Anderson O.S., Sant K.E., Dolinoy D.C. (2012). Nutrition and epigenetics: An interplay of dietary methyl donors, one-carbon metabolism and DNA methylation. Journal of Nutritional Biochemistry, 23 (8), 853-859.

Anway M.D., Cupp A.S., Uzumcu M., Skinner M.K. (2005). Epigenetic transgenera-tional actions of endocrine disruptors and male fertility. Science, 308, 1466-1469.

Araten D.J., Nafa K., Pakdeesuwan K., Luzzatto L. (1999). Clonal populations of hematopoietic cells with paroxysmal nocturnal hemoglobinuria genotype and phenotype are present in normal individuals. Proceedings of the National Academy of Science U.S.A., 96, 5209-5214.

Araten D.J., Climentc J.A.M., Perled M.A., Holma E., Zamecheka L., Tataa K.D., Sanders K.J. (2010). A quantitative analysis of genomic instability in lymphoid and plasma cell neoplasms based on the PIG-A gene. Mutation Research, 686, 1-8.

Auerbach P., Bennett R.A., Bailey E.A., Krokan H.E., Demple B. (2005). Mutagenic specificity of endogenously generated abasic sites in saccharomyces cerevisiae chromosomal DNA. Proceedings of the National Academy of Science USA, 102, 17711-17716. 
Ba Y., Yu H., Liu F., Geng X., Zhu C., Zhu Q., Zheng T., Ma S., Wang G., Li Z., Zhang Y. (2011). Relationship of folate, vitamin $B_{12}$ and methylation of insulin-like growth factor-II in maternal and cord blood. European Journal of Clinical Nutrition, 65 (4), 480-485.

Bagnyukova T.V., Powel C.L., Pavliv O., Tryndyak V.P., Pogribny I.P. (2008). Induction of oxidative stress and DNA damage in rat brain by a folate/methyl deficient diet. Brain Research, 1237, 44-51.

Bailey L.B., Gregory J.F. (1999). Folate metabolism and requirements. Journal of Nutrition, 129, 779-782.

Bailey S.W., Ayling J.E. (2009). The extremely slow and variable activity of dihydrofolate reductase in human lover and its implication for high folic acid intake. Proceedings of the National Academy of Sciences USA, 106 (36), 15424-15429.

Balaghi M., Wagner C. (1993). DNA methylation in folate deficiency: use of CpG methylase. Biochemical and Biophysical Research Communications, 193, 1184-1190.

Balhorn R., Gledhill B.L., Wyrobek A. (1977). Mouse sperm chromatin proteins: quantitative isolation and partial characterization. Biochemistry, 16, 4074-4080.

Bandera E.V., Freudenheim J.L., Marshall J.R., Zielezny M., Priore R.L., Brasure J., Baptiste M., Graham S. (1997). Diet and alcohol consumption and lung cancer risk in the New York state cohort. Cancer Causes and Control, 8 (6), 828-840.

Banerjee A., Quirke P. (1998). Experimental models of colorectal cancer. Diseases of the Colon and Rectum, 41 (4), 490-505.

Barber R.C., Plumb M., Smith A.G., Cesar C.E., Boulton E., Jeffreys A.J., Dubrova Y.E. (2000). No correlation between germline mutation at repeat DNA and meiotic crossover in male mice exposed to $\mathrm{X}$-rays or cisplatin. Mutation Research/ Fundamental and Molecular Mechanisms of Mutagenesis, 457, 79-91.

Barber R.C., Plump M.A., Boulton E., Roux I., Dubrova Y.E. (2002). Elevated mutation rates in the germline of first- and second-generation offspring of irradiated male mice. Proceedings of the National Academy of Sciences USA, 99, 6877-6882.

Barber R.C., Dubrova Y.E. (2006a). The offspring of irradiated parents, are they stable? Mutation Research, 598, 50-60. 
Barber R.C., Hickenbotham P., Hatch T., Kelly D., Topchiy N., Almeida G., Jones G.G.D., Johnson G.E., Parry J.M., Rothkamm K., Dubrova Y.E. (2006b). Radiation-induced transgenerational alterations in genome stability and DNA damage. Oncogene, 25, 7336-7342.

Barber R.C., Hardwick R.J., Shanks M.E., Glen C.D., Mughal S.K., Voutounou M. (2009). The effects of in utero irradiation on mutation induction and transgenerational instability in mice. Mutation Research, 664, 6-12.

Bashir O., FitzGerald A., Goodlad R. (2004). Both suboptimal and elevated vitamin intake increase intestinal neoplasia and alter crypt fission in the $\mathrm{Apc}^{\mathrm{Min} /+}$ mouse. Carcinogenesis, 25, 1507-1515.

Basten G.P., Duthie S.J., Pirie L., Vaughan N., Hill M.H., Powers H.J. (2006). Sensitivity of markers of DNA stability and DNA repair activity to folate supplementation in healthy volunteers. British Journal of Cancer, 94, 19421947.

Becker F.F., Holton P., Ruchirawat M., Lapeyre J.N. (1985). Perturbation of maintenance and de novo DNA methylation in vitro by UVB (280-340 nm)induced pyrimidine photodimers. Proceedings of the National Academy of Sciences USA, 82, 6055-6059.

Beetstra S., Thomas P., Salisbury C., Turner J., Fenech M. (2005). Folic acid deficiency increases chromosomal instability, chromosome 21 aneuploidy and sensitivity to radiation-induced micronuclei. Mutation Research, 578, 317-326.

Biermann K., Steger K. (2007). Epigenetics in male germ cells. Journal of Andrology, 28 (4), 466-480.

Bistulfi G., VanDette E., Matsui S.I., Smiraglia D.J. (2010). Mild folate deficiency induces genetic and epigenetic instability and phenotype changes in prostate cancer cells. BioMed Central Biology, 8, 6.

Blewitt M. E., Vickaryous N. K., Paldi A., Koseki H., Whitelaw E. (2006). Dynamic reprogramming of DNA methylation at an epigenetically sensitive allele in mice. Plos Genetics, 2, 49.

Block G., Patterson B., Subar A. (1992). Fruit, vegetables and cancer prevention: a review of the epidemiologic evidence. Nutritional Cancer, 18, 129.

Blount B.C., Mack M.M., Wehr C.M., MacGregor J.T., Hiatt R.A., Wang G. (1997). Folate deficiency causes uracil misincorporation into human DNA and chromosome break- age: implications for cancer and neuronal damage. Proceedings of the National Academy of Science USA, 94, 3290-3295.

Blusztajn J. K. (1998). Choline, a vital amine. Science, 281, 794-795. 
Bois P., Willian J., Brown J., Dubrova Y.E., Jeffreys A.J. (1998). A novel unstable mouse VNTR family expanded from SINE B1 element. Genomics, 49, 122-128.

Boland M.J., Christman J.K. (2008). Characterization of Dnmt3b:thymineDNA glycosylase interaction and stimulation of thymine glycosylase-mediated repair by DNA methyltransferase(s) and RNA. Journal of Molecular Biology, 379, 492-504.

Bonassi S., Hagmar L., Strömberg U., Montagud A.H., Tinnerberg H., Forni A., Heikkilä P., Wanders S., Wilhardt P., Hansteen I.L., Knudsen L.E., Norppa H. (2000). Chromosomal aberrations in lymphocytes predict human cancer independently of exposure to carcinogens. European study group on cytogenetic biomarkers and health. Cancer Research, 60, 1619-1625.

Boushey C.J., Beresford S.A.A., Omenn G.S., Motulsky A.G. (1995). A quantitative assessment of plasma homocysteine as a risk factor for vascular disease: probable benefits of increasing folic acid intakes. Journal of American Medical Association, 274, 1049-1057.

Boxmeer J.C., Smit M., Utomo E., Romijn J.C., Eijkemans M.J., Lindemans J. Laven J.S., Macklon N.S., Steegers E.A., Steegers-Theunissen R.P. (2009). Low folate in seminal plasma is associated with increased sperm DNA damage. Fertility and Sterility, 92, 548-556.

Brandt C.J., Rutherford D. (2007). Vitamins, minerals and supplements. NetDoctor.co.uk. http://www.netdoctor.co.uk/health_advice/facts/vitamins.htm (assessed 17-06-12).

Bryce S.M., Bemis J.C., Dertinger S.D. (2008). In vivo mutation assay based on the endogenous Pig-a locus. Environmental and Molecular Mutagenesis, 49(4), 256-264.

Bull C.F., Mayrhofer G., Zeegers D., Mun G.L.K., Hande M.P., Fenech M.F. (2012). Folate deficiency is associated with the formation of complex nuclear anomalies in the cytokinesis-block micronucleus cytome assay. Environmental and Molecular Mutagenesis, 53, 311-323.

Burdge G.C., Lillycrop K.A., Phillips E.S., Slater-Jefferies J.L., Jackson A.A., Hanson M.A. (2009). Folic acid supplementation during the juvenile-pubertal period in rats modifies the phenotype and epigenotype induced by prenatal nutrition. Journal of Nutrition, 139 (6), 1054-1060.

Burgoon J.M., Selhub J., Nadeau M., Sadlter T.W. (2002). Investigation of the effects of folate deficiency on embryonic development through the establishment of a folate deficient mouse model. Teratology, 65, 219-227.

Burr K.L.A., Smith A.G., Dubrova Y.E. (2005). P53 deficiency does not affect mutation rate in the mouse germline. Oncogene, 24, 4315-4318. 
Carroll N., Hughes L., McEntee G., Parle-McDermott A. (2012). Investigation of the molecular response to folate metabolism inhibition. Journal of Nutritional Biochemistry, 1, 1-6.

Caudill M.A., Wang J.C., Melnyk S., Pogribny I.P., Jernigan S., Collins M.D., Guzman J.S., Swendseid M.E., Cogger E.A., James S.J. (2001). Sadenosylhomocysteine concentrations predict global DNA hypomethylation in tissues of methyl-deficient cystathionine $\beta$-synthase heterozygous mice. Journal of Nutrition, 131, 2811-2818.

Champier J., Claustrat F., Nazaret N., Montange F.M., Claustrat B. (2012). Folate depletion changes gene expression of fatty acid metabolism, DNA synthesis, and circadian cycle in male mice. Nutrition Research, 32 (2), 124132.

Chan D., Cushnie D.W., Neaga O.R., Lawrance A.K., Rozen R., Trasler J.M. (2010). Strain-specific defects in testicular development and sperm epigenetic patterns in 5,10-methylenetetrahydrofolate reductase-deficient mice. Endocrinology, 151, 3363-3373.

Chang H.S., Anway M.D., Rekow S.S., Skinner M.K. (2008). Transgenerational epigenetic imprinting of the male germline by endocrine disruptor exposure during gonad sex determination. Endocrinology, 147 (12), 5524-5541.

Chatenoud L., Tavani A., La Vecchia C., Jacobs D.R., Negri E., Levi F., Franceschi S. (1998). Whole grain food intake and cancer risk. International Journal for Cancer, 77, 24-28.

Chiang P. K., Gordon R. K., Tal J., Zeng G. C., Doctor B. P., Pardhasaradhi K., McCann P.P. (1996). S-adenosylmethionine and methylation. Federation of American Societies for Experimental Biology, 10, 471-480.

Choi S.W., Kim Y.I., Weitzel J.N., Mason J.B. (1998). Folate depletion impairs DNA excision repair in the colon of rat. International Journal of Gastroenterology and Hepatology, 43, 93-99.

Choi S.W., Friso S., Dolnikowski G.G., Bagley P.J., Edmondson A.N., Smith D.E., Mason J.B. (2003). Biochemical and molecular aberrations on the rat colon due to folate depletion are age-specific. Journal of Nutrition, 133, 12061212.

Chen A.T.L., Reidy J.A., Annest J.L., Welty T.K., Zhou H. (1989). Increased chromosome fragility as a consequence of blood folate levels, smoking status and coffee consumption, Environmental and Molecular Mutagenesis, 13, 319-324. 
Christensen B.C., Kelsey K.T., Zheng S., Houseman E.A., Marsit C.J., Wrensch M.R., Wiemels J.L., Nelson H.H., Karagas M.R., Kushi L.H., Kwan M.L., Wiencke J.K. (2010). Breast cancer DNA methylation profiles are associated with tumor size and alcohol and folate intake. Public Library of Science Genetics, 6 (7), 1-10.

Christensen K.E., Mason J.B., Rozen R. (2011). In: Vitamins in the prevention of human diseases. Herrmann W., Obeid R. (eds). De Gruyter W., Berlin, pp. 91-186.

Christman J.K. (2003). In: Molecular nutrition. Diet, DNA methylation and cancer. Zempleni J., Daniel H. (eds). CABI, Oxon, pp. 237-265.

Christman J.K. (1995). Lipotrope deficiency and persistent changes in DNA methylation. Advances in Experimental Medicine and Biology, 375, 97-106.

Ciappio E.D., Mason J.B. (2010). In: Folate in health and disease. Folate and carcinogenesis: basic mechanisms. Bailey L.B. (ed). Taylor-Francis, New York, pp. 235-62.

Cole B.F., Baron J.A., Sandler R.S., Haile R.W., Ahnen D.J., Bresalier R.S., McKeown-Eyssen G., Summers R.W., Rothstein R.I., Burke C.A., Snover D.C., Church T.R., Allen J.I., Robertson D.J., Beck G.J., Bond J.H. (2007). Folic acid for the prevention of colorectal adenomas: a randomized clinical trial. Journal of American Medical Association, 297, 2351-2359.

Creusot F., Acs G., Christman J.K. (1982). Inhibition of DNA methyltransferase and induction of friend erythroleukaemia cell differentiation by 5-azacytidine and 5-aza-2-deoxycytidine. Journal of Biology and Chemistry, 257, 2041-2048.

Cropley J.E., Suter C.M., Beckman K.B., Martin D.I.K. (2006). Germ-line epigenetic modification of the murine $A^{v y}$ allele by nutritional supplementation. Proceedings of the National Academy of Science USA, 103, 17308-17312.

Crott J.W., Mashiyama S.T., Ames B.N., Fenech M.F. (2001). Methylenetetrahydrofolate reductase C677T polymorphism does not alter folic acid deficiency-induced uracil incorporation into primary human lymphocyte DNA in vitro. Carcinogenesis, 22, 1019-1025.

Crott J.W., Liu Z., Choi S.W., Mason J.B. (2007). Folate depletion in human lymphocytes up-regulates p53 expression despite marked induction of strand breaks in exons 508 of the gene. Mutation Research, 626, 171-179.

Czeizel A.E., Dudas I. (1992). Prevention of the first occurrence of neural tube defects by periconceptional vitamin supplementation. New England Journal of Medicine, 327, 1832-1835. 
Daly S., Mills J.L., Molloy A.M., Lee Y.J., Kirke P.N., Weir D.G., Scott J.M. (1997). Minimum effective dose of folic acid for food fortification to prevent neural-tube defects. Lancet, 350, 1666-1669.

Daly L.E., Kirke P.N., Molloy A., Weir D.G., Scott J.M. (1995). Folate levels and neural tube defects implications for prevention. Journal of American Medical Association, 274, 1698-1702.

Daura-Oller E., Cabre M., Montero M.A., Paternain J.L., Romeu A. (2009). Specific gene hypomethylation and cancer: New insights into coding region feature trends. Biomedical Informatics Publishing Group, 3 (8), 340-343.

Davis C.D., Uthus E.O. (2004). DNA methylation, cancer susceptibility and nutrient interactions. Experimental Biology and Medicine, 229, 988-995.

Davis T.L., Yang G.J., McCarrey J.R., Bartolomei M.S. (2000). The H19 methylation imprint is erased and re-established differentially on the parental alleles during male germ cell development. Human Molecular Genetics, 9, 2885-2894.

Davison J.M., Mellott T.J., Kovacheva V.P., Blusztajn J.K. (2008). Gestational choline supply regulates methylation of histone $\mathrm{H} 3$, expression of histone methyltransferases G9A (KMT1C) and SUV39H1 (KMT1A) and DNA methylation of their genes in rat foetal liver and brain. Journal of Biology and Chemistry, 1, 1-11.

Daxinger L., Whitelaw E. (2012). Understanding transgenerational epigenetic inheritance via the gametes in mammals. Nature Reviews, 13, 153-162.

De Rooij D.G., Russell L.D. (2000). All you wanted to know about spermatogonia but were afraid to ask. Journal of Andrology, 21, 776-798.

De Vos L., Chanson A., Liu Z., Ciappio E.D., Pamell L.D., Mason J.B., Tucker K.L., Crott J.W. (2008). Associations between single nucleotide polymorphisms in folate uptake and metabolizing genes with blood folate, homocystein, and DNA uracil concentrations. American Journal of Clinical Nutrition, 88, 1149-1158.

De Wals P., Tairou F., Van Allen M.I., Uh S.H., Lowry R.B., Sibbald B., Evans J.A., Van den Hof M.C., Zimmer P., Crowley M., Fernandez B., Lee N.S., Niyonsenga T. (2007). Reduction in neural tube defects after folic acid fortification in Canada. The New England Journal of Medicine, 357, 135-142.

Dertinger S.D., Phonethepswath S., Franklin D., Weller P., Torous D.K., Bryce S.M., Avlasevich S., Bemis J.C., Hyrien O., Palis J.C., MacGregor J.T. (2010). Integration of Mutation and Chromosomal Damage Endpoints into 28Day Repeat Dose Toxicology Studies. Toxicological Sciences, 115, 401-411. 
Dianov L.M., Timchenko T.V., Sinitsina O.I., Kuzminov A.V., Medvedev O.A., Salganik R.I. (1991). Repair of uracil residues closely spaced on the opposite strands of plasmid DNA results in double-strand break and deletion formation. Molecular Genetics and Genomics, 225, 448-452.

Discombe G. (1948). L'origine des corps de Howell-Jolly et des anneaux de cabot. Sangre, 29, 262-270.

Dobrovolsky V.N., Boctor S.Y., Twaddle N.C., Doerge D.R., Bishop M., Manjanatha M.G., Kimoto T., Miura D., Heflich R.H., Ferguson S.A. (2009). Flow cytometric detection of Pig-A mutant red blood cells using an erythroidspecific antibody: Application of the method for evaluating the in vivo genotoxicity of methylphenidate in adolescent rats. Environmental and Molecular Mutagenesis, 1,1-8.

Dobrovolsky V.N., Boctor S.Y.,.Twaddle N.C., Doerge D.R., Bishop M.E., Manjanatha M.G., Kimoto T., Miura D., Heflich R.H., Ferguson S.A. (2010a). Flow Cytometric Detection of Pig-A Mutant Red Blood Cells Using an Erythroid-Specific Antibody: Application of the Method for Evaluating the In Vivo Genotoxicity of Methylphenidate in Adolescent Rats. Environmental and Molecular Mutagenesis, 51, 138-145.

Dobrovolsky V.N., Miura D., Heflich R., Dertinger S.D. (2010b). The In Vivo Pig-a Gene Mutation Assay, A Potential Tool For Regulatory Safety Assessment. Environmental and Molecular Mutagenesis, 1, 1-11.

Dodge J.E., Ramsahoye B.H., Wo Z.G., Okano M., Li E. (2002). De novo methylation of MMLV provirus in embryonic stem cells: CpG versus non-CpG methylation. Gene, 289 (2), 41-48.

Du Y.P., Peng J.S., Sun A., Tang Z.H., Ling W.H., Zhu H.L. (2009). Assessment of the effect of betaine on $p 16$ and c-myc DNA methylation and mRNA expression in a chemical induced rat liver cancer model. Journal of Negative Results in Biomedicine, 9 (1), 261.

Dubrova Y.E., Jeffreys A.J., Malashenko A.M. (1993). Mouse minisatellite mutations induced by ionizing radiation. Nature Genetics, 5, 92-94.

Dubrova Y.E., Plumb M., Brown J., Fennelly J., Bois P., Goodhead D., Jeffreys A.J. (1998). Stage specificity, dose response, and doubling dose for mouse minisatellite germ-line mutation induced by acute radiation. Proceedings of the National Academy of Science USA, 95, 6251-6255.

Dubrova Y.E., Plumb M., Brown J., Boulton J., Goodhead D., Jeffreys A.J. (2000a). Induction of minisatellite mutations in the mouse germline by lowdose chronic exposure to $\gamma$-radiation and fission neutrons. Mutation Research, 453, 17-24. 
Dubrova, Y.E., Plumb, M., Gutierrez, B., Boulton, E. and Jeffreys, A.J. (2000b). Transgenerational mutation by radiation. Nature, 405, 37.

Dubrova Y.E. (2003). Radiation-induced transgenerational instability. Oncogene, 22, 7087-7093.

Dubrova Y.E. (2005a). Mutation induction in mice: reduction in practice. http://www.nc3rs.org.uk/news.asp?id=118 (accessed 06-22-2012).

Dubrova Y.E. (2005b). Radiation-induced mutation at tandem repeat DNA loci in the mouse germline: Spectra and doubling doses. Radiation Research, 163, 200-207.

Dubrova Y.E., Hickenbotham P., Glen C.D., Monger K., Wong H.P., Barber R.C. (2008). Paternal exposure to ethylnitrosourea results in transgenerational genomic instability in mice. Environmental Molecular Mutagenesis, 49, 308-311.

Dunn B.K. (2003). Hypomethylation: one side of a larger picture. Annals of the New York Academy of Sciences, 983, 28-42.

Duthie S.J., Hawdon A. (1998). DNA instability (strand breakage, uracil misincorporation, and defective repair) is increased by folic acid depletion in human lymphocytes in vitro. FASEB Journal, 12, 1491-1497.

Duthie S.J. (1999). Folic acid deficiency and cancer: mechanisms of DNA instability. British Medical Bulletin, 55 (3), 578-592.

Duthie S.J., Grant G., Narayanan S. (2000a). Increased uracil misincorporation in lymphocytes from folate-deficient rats. British Journal of Cancer, 83, 1532-1537.

Duthie S.J., Narayanan S., Blum S., Pirie L., Brand G.M. (2000b). Folate deficiency in vitro induces uracil misincorporation and DNA hypomethylation and inhibits DNA excision repair in immortalized normal human colon epithelial cells. Nutrition and Cancer, 37 (2), 245-251.

Duthie S.J., Narayanan S., Brand G.M., Grant G. (2000c). DNA stability and genomic methylation status in colonocytes isolated from methyl- donordeficient rats. European Journal for Nutrition, 39, 106-111.

Duthie S.J., Narayanan S., Brand G.M., Pirie L., Grant G. (2002). Impact of folate deficiency on DNA stability. The American Society for Nutritional Sciences, 1, 2444-2449.

Duthie S.J., Narayanan S., Sharp L., Little J., Basten G., Powers H. (2004). Folate, DNA stability and colorectal neoplasia. Proceedings of the Nutrition Society, 63, 571-578.

Duthie S.J., Grant G., Pirie L.P., Watson A.J., Margison G.P. (2010). Folate deficiency alters hepatic and colon MGMT and OGG-1 DNA repair protein 
expression in rats but has no effect on genome-wide DNA methylation. Cancer Prevention Research, 3 (1), 92-100.

Eden A., Gaudet F., Waghmare A., Jaenisch R. (2003). Chromosomal instability and tumours promoted by DNA hypomethylation. Science, 300, 455.

Ellis C., Macleod A.L. (1922). In: Vital Factors Of Foods: Vitamins And Nutrition. Van Nostrand D. Company, New York, pp. 66-88.

Ellis M. (2008) Amino acids, methionine: An essential sulphur-containing amino acid. Health News, http://www.healthnews.com/natural-health/aminoacids.

Eichholzer M., Luthy J., Moser U., Fowler B. (2001). Folate and the risk of colorectal, breast and cervix cancer: the epidemiological evidence. Swiss Medical Weekly, 131, 539-549.

Ericson U., Sonestedt E., Gullberg B., Olsson H., Wirfalt E. (2007). High folate intake is associated with lower breast cancer incidence in postmenopausal women in the Malmo diet and cancer cohort. American Journal of Clinical Nutrition, 86, 434-443.

Esteller M. (2007). Cancer epigenomics: DNA methylomes and histonemodification maps. Nature Reviews Genetics, 8, 286-297.

Eto I., Krumdieck C.L, Poirier L.A., Newberne P.M., Pariza M.W. (1986). Role of vitamin $B_{12}$ and folate deficiencies in carcinogenesis. Essential nutrients in carcinogenesis. Plenum Press, 1, 313-331.

Everson R.B., Wehr C.M., Erexson G.L., MacGregor J.T. (1988). Association of marginal folate depletion with increased human chromosomal damage in vivo: demonstration by analysis of micronucleated erythrocytes. Journal of National Cancer Institute, 80, 525-529.

Falnes P.O., Johansen R.F., Seeberg E. (2002). AlkB-mediated oxidative demethylation reverses DNA damage in Escherichia coli. Nature, 419, 17882.

Fan T., Schmidtmann A., Xi S., Briones V., Zhu H., Suh H.C., Gooya J., Keller J.R., Xu H., Roayaei J., Anver M., Ruscetti S., Muegge K. (2008). DNA hypomethylation caused by LSH deletion promotes erythroleukemia development. Epigenetics, 3, 134-142.

Farlex, Inc. (2010). The free dictionary: Definition of nutrition in the Medical dictionary. http://medical-dictionary.thefreedictionary.com/nutrition (assessed 16-04-212).

Favor J., Neuhäuser-Klaus A. (1994). Genetic mosaicism in the house mouse. Annual Review of Genetics, 28, 27-47. 
Feinberg A.F. (2007). Phenotypic plasticity and the epigenetics of human disease. Nature, 447, 433-440.

Feinberg A. P., Vogelstein B. (1983). A technique for radiolabeling DNA restriction endonuclease fragments to high specific activity. Analytical Biochemistry, 132, 6-13.

Feinberg A. P., Vogelstein B. (1984). A technique for radiolabeling DNA restriction endonuclease fragments to high specific activity. Addendum. Analytical Biochemistry, 137, 266-267.

Feinberg A.P., Ohlsson R., Henikoff S. (2006). The epigenetic promoter origin of human cancer. Nature Reviews Genetics, 7, 21-33.

Fenech M.F., Dreosti I.E., Rinaldi J.R. (1997). Folate, Vitamin B B $_{12}$ homocysteine status and chromosome damage rate in lymphocytes of older men. Carcinogenesis, 18, 1329-1336.

Fenech M., Aitken C., Rinaldi J. (1998). Folate, vitamin $B_{12}$, homocystein status and DNA damage in young Australian adults. Carcinogenesis, 19, 116-171.

Fenech M. (2001). The role of folic acid and vitamin $B_{12}$ in genomic stability of human cells. Mutation Research, 475, 57-67.

Fenech M. (2012). Folate (vitamin $B_{9}$ ) and vitamin $B_{12}$ and their function in the maintenance of nuclear and mitochondrial genome integrity. Mutation Research, 733, 21-33.

Fenech M., Kirsch-Volders M., Natarajan A.T., Surralles J., Crott J.W., Parry J., Norppa H., Eastmond D.A., Tucker J.D., Thomas P. (2011). Molecular mechanisms of micronucleus, nucleoplasmic bridge and nuclear bud formation in mammalian and human cells. Mutagenesis, 26, 125-32.

Figueiredo J.C., Grau M.V., Wallace K., Levine A.J., Shen L., Hamdan R., Chen X., Bresalier R.S., McKeown-Eyssen G., Hail R.W., Baron J.A., Issa J.P.J. (2009). Global DNA hypomethylation (LINE-1) in the normal colon and lifestyle characteristics, dietary and genetic factors. Cancer Epidemiology Biomarkers and Prevention, 18 (4), 1041-1049.

Finkelstein J. D., Martin J. J., Harris B. J., Kyle, W. E. (1983). Regulation of hepatic betaine-homocysteine methyltransferase by dietary betaine. Journal of Nutrition, 113, 519-521.

Flanagan J. M., Popendikyte V., Pozdniakovaite N., Sobolev M., Assadzadeh A., Schumacher A., Zangeneh M., Lau L., Virtanen C., Wang S.C., Petronis A. (2006). Intra- and inter-individual epigenetic variation in human germ cells. American Journal of Human Genetics, 79, 67-84. 
Fodde R., Smits R., Clevers H. (2001). Apc, signal transduction and genetic instability in colorectal cancer. National Reviews of Cancer, 1 (1), 55-67.

Fodde R. (2002). The Apc gene in colorectal cancer. European Journal of Cancer, 38 (7), 867-871.

Fu S.S., Sakanashi T.M., Rogers J.M., Hong K.H., Keen C.L. (1996). Influence of dietary folic acid on the developmental toxicity of methanol and frequency of chromosomal breakage in the CD-1 mouse. Reproductive Toxicology, 10, 455-463.

Fukuda, H., Katahira M., Tsuchiya N., Enokizono Y., Sugimura T., Nagao M., Nakagama H. (2002). Unfolding of quadruplex structure in the G-rich strand of the minisatellite repeat by the binding protein UP1. Proceedings of the National Academy of Sciences USA, 99, 12685-12690.

Fulka H., Mrazek M., Tepla O., Fulka J. (2004). DNA methylation pattern in human zygotes and developing embryos. Reproduction, 128, 703-708.

Gatewood J.M., Cook G.R., Balhorn R., Bradbury E.M., Schmid C.W. (1987). Sequence-specific packaging of DNA in human sperm chromatin. Science, 236, 962-964.

Gatewood J.M., Cook G.R., Balhorn R., Schmid C.W., Bradbury E.M. (1990). Isolation of 4 core histones from human sperm chromatin representing a minor subset of somatic histones. Journal of Biological Chemistry, 265, 20662-20666.

Gibbs M., Collick A., Kelly R. G., Jeffreys A. J. (1993). A tetranucleotide repeat mouse minisatellite displaying substantial somatic instability during early preimplantation development. Genomics, 17, 121-128.

Giovannucci E., Rimm E.B., Ascherio A., Stampfer M.J., Colditz G.A., Willett W.C. (1995). Alcohol, methyl-deficient diets and risk of colon cancer in men. Journal of the National Cancer Institute, 87, 265-273.

Giovannucci E., Stampfer M.J., Colditz G.A., et al. (1998). Multivitamin use, folate, and colon cancer in women in the Nurses' Health Study. Annals of Internal Medicine, 129, 517-524.

Glen C.D., Smith A.G., Dubrova Y.E. (2008). Single-molecule PCR analysis of germ line mutation induction by anticancer drugs in mice. Cancer Research, 68, 3630-3636.

Glen C.D., Dubrova Y.E. (2012). Exposure to anticancer drugs can result in transgenerational genomic instability in mice, Proceedings of the National Academy of Science, U.S.A., 109, 1-8. 
Glenn M. J., Gibson E. M., Kirby E. D., Mellott T. J., Blusztajn J. K., Williams, C. L. (2007). Prenatal choline availability modulates hippocampal neurogenesis and neurogenic responses to enriching experiences in adult female rats. European Journal of Neuroscience, 25, 2473-2482.

Glenn M. J., Kirby E. D., Gibson E. M., Wong-Goodrich S., Mellott T. J., Blusztajn J. K., Williams C. L. (2008). Age related declines in exploratory behavior and markers of hippocampal plasticity are attenuated by prenatal choline supplementation in rats. Brain Research, 1237, 110-123.

Goll M.G., Kirpekar F., Maggert K.A., Yoder J.A., Hsieh C.L., Zhang X., Golic K.G., Jacobsen S.E., Bestor T.H. (2006). Methylation of tRNAAsp by the DNA methyltransferase homolog Dnmt2. Science, 311(5759), 395-398.

Goodman, M. T., McDuffie K., Hernandez B., Wilkens L.R., Bertram C.C., Killeen J., Marchand L.L., Selhub J., Murphy S., Dolon T.A. (2001). Association of methylene-tetrahydrofolate reductase polymorphism C677T and dietary folate with the risk of cervical dysplasia. Cancer Epidemiology Biomarkers and Prevention, 12, 1275-1280.

Gorbunova V., Seluanov A., Mao Z., Hine C. (2007). Changes in DNA repair during aging. Nucleic Acid Research, 35, 7466-7474.

Goulian M., Bleile B., Tseng B.Y. (1980). Methotrexate-induced misincorporation of uracil into DNA. Proceedings of the National Academy of Sciences USA, 77 (4), 1956-1960.

Groden J., Thliveris A., Samowitz W., Carlson M., Gelbert L., Albertsen H., Joslyn G., Stevens J., Spirio L., Robertson M. (1991). Identification and characterization of the familial adenomatous polyposis coli gene. Cell, 66, 589-600.

Haines T.R., Rodenhiser D.I., Ainsworth P.J. (2001). Allele-specific non-CpG methylation of the Nf1 gene during early mouse development. Developmental Biology, 240 (2), 585-598.

Hales B.F., Grenier L., Lalancette C., Robaire B. (2011). Epigenetic programming: from gametes to blastocyst. Birth Defects Research: Clinical and Molecular Teratology, 91 (8), 652-665.

Hardwick R.J., Tretyakov M.V., Dubrova Y.E. (2009). Age-related accumulation of mutations supports a replication-dependent mechanism of spontaneous mutation at tandem repeat DNA loci in mice. Molecular Biology and Evolution, 26, 2647-2654.

Harris H.R., Bergkvist L., Wolk A. (2012). Folate intake and breast cancer mortality in a cohort of Swedish women. Breast Cancer Research and Treatment, 132 (1), 243-250. 
Hatch T., Derijck A.A.H.A., Black P.D., Van der Heijden G.W., De Boer Y., Dubrova Y.E. (2007). Maternal effects of the scid mutation on radiationinduced transgenerational instability in mice. Oncogene, 26, 4720-4724.

Hazra A., Wu K., Kraft P., Fuchs C.S., Giovannucci E.L., Hunter D.J. (2007). Twenty-four non-synonymous polymorphisms in the one-carbon metabolic pathway and risk of colorectal adenoma in the nurses' health study. Carcinogenesis, PubMed, 8 (7), 1510-1519.

Hazra A., Selhub J., Chao W.H., Ueland P.M., Hunter D.J., Baron J.A. (2010). Uracil misincorporation into DNA and folic acid supplementation. American Journal of Clinical Nutrition, 91, 160-165.

Herman J.G., Graff J.R., Myohanen S., Nelkin B.D., Baylin S.B. (1996). Methylation- specific PCR: A novel PCR assay for methylation status of CpG islands. Proceedings of National Academy of Science, 87, 7160-4.

Hervouet E., Debien E., Campion L., Charbord J., Menanteau J., Vallette F.M., Cartron P.F. (2009). Folate supplementation limits the aggressiveness of glioma via the remethylation of DNA repeats element and genes governing apoptosis and proliferation. Clinical Cancer Research, 15 (10), 3519-3529.

Hoyo C., Murtha A.P., Schildkraut J.M., Jirtle R., Demark-Wahnefried W., Forman M.R., Iversen E.S., Kurtzberg J., Overcash F., Zhiqing H., Murphy S.K. (2011). Methylation variation at IGF2 differentially methylated regions and maternal folic acid use before and during pregnancy. Epigenetics, 6 (7), 928-936.

Howard G., Eiges R., Gaudet F., Jaenisch R., Eden A. (2008). Activation and transposition of endogenous retroviral elements in hypomethylation induced tumours in mice. Oncogene, 27 (3), 404-408.

Jackson K., Yu M.C., Arakawa K., Fiala E., Youn B., Fiegl H., Muller-Holzner E., Widschwendter M., Ehrlich M. (2004). DNA hypomethylation is prevalent in low-grade breast cancers. Cancer Biological Therapy, 3, 1225-1231.

Jacobs D.R., Marquart L., Slavin J., Kushi L.H. (1998). Whole-grain intake and cancer: an expanded review and meta-analysis. Nutritional Cancer, 30, 85-96.

Jacobs E.J., Connell C.J., Chao A., McCullough M.L., Rodriguez C., Thun M.J., Calle E.E. (2003). Multivitamin use and colorectal cancer incidence in a US cohort: does timing matter? American Journal of Epidemiology, 158 (7), 621-628.

Jamaluddin M.D., Yang X., Wang H. (2007). Hyperhomocysteinemia, DNA methylation and vascular disease. Clinical Chemistry and Laboratory Medicine, 45, 1660-1666. 
James S.J., Basnakian A.G., Miller B.J. (1994). In vitro folate deficiency induces deoxynucleotide pool imbalance, apoptosis, and mutagenesis in Chinese hamster ovary cells. Cancer research, 54, 5057-5080.

James S.J., Pogribny I.P., Pogribna M., Miller B.J., Jernigan S., Melnyk S. (2003). Mechanisms of DNA damage, DNA hypomethylation, and tumour progression in the folate/methyl-deficient rat model of hepatocarcinogenesis. Journal of Nutrition, 133, 3740-3747.

Jasperson K.W., Samowitz W.S., Burth R.W. (2010). Constitutional mismatch repair-deficiency syndrome presenting as colonic adenomatous polyposis: clues from the skin. Clinical Genetics, 80 (4), 394-397.

Jeffreys A.J., MacLeod A., Tamaki K., Neil D.L., Monckton D.G. (1991). Minisatellite repeat coding as a digital approach to DNA typing. Nature, 354, 204-209.

Jones J.P., Meck H.W., Williams C.L., Wilson W.A., Swartzwelder H.S. (1999). Choline availability to the developing rat fetus alters adult hippocampal long-term potentiation. Developmental Brain Research, 118, 159-167.

Jones P.A. (2002). DNA methylation and cancer. Oncogene, 21, 5358-5360.

Jost J.P. (1993). Nuclear extracts of chicken embryos promote an active demethylation of DNA by excision repair of 5-methyldeoxycytidine. Proceedings of the National Academy of Science U.S.A., 90, 4684-4688.

Jost J.P., Siegmann M., Sun L., Leung R. (1995). Mechanisms of DNA demethylation in chicken embryos. Purification and properties of a 5methylcytosine-DNA glycosylase. Journal of Biology and Chemistry, 270, 9734-9739.

Kaminsky Z.A., Tang T., Wang S.C., Ptak C., Oh G.H., Wong A.H., Feldcamp L.A., Virtanen C., Halfvarson J., Tysk C., McRae A.F., Visscher P.M., Montgomery G.W., Gottesman I.I., Martin N.G., Petronis A. (2009). DNA methylation profiles in monozygotic and dizygotic twins. Nature Genetics, 41, 240-245.

Kapiszewska M., Kalemba M., Wojciech U., Milewicz T. (2005). Uracil misincorporation into DNA of leukocytes of young women with positive folate balace depends on plasma vitamin $\mathrm{B}_{12}$ concentrations and methyltetrahydropholate reductase polymorphisms. A pilot study. Journal of Nutritional Biochemistry, 16, 467-478.

Kazazian H.H. (1999). An estimated frequency of endogenous insertional mutations in humans. Nature Genetics, 22, 130. 
Kelly R., Bulfield G., Collick A., Gibbs M., Jeffreys A.J. (1989). Characterization of a highly unstable mouse minisatellite locus: evidence for somatic mutation during early development. Genomics, 5, 844-856.

Kelly R., Gibbs M., Collick A., Jeffreys A.J. (1991). Spontaneous mutation at the hypervariable mouse minisatellite locus Ms6-hm - Flanking DNAsequence and analysis of germline and early somatic mutation events. Proceedings of the Royal Society of London Series Biological Sciences, 245, 235-245.

Kendler K.S., Baker J.H. (2007). Genetic influences on measures of the environment a systematic review. Psychology and Medicine, 37, 615-626.

Keszei A.P., Verhage B.A.J., Heinen M.M. (2009). Dietary folate and folate vitamers and the risk of pancreatic cancer in the Netherlands cohort study. Cancer Epidemiology Biomarkers and Prevention, 18 (6), 1785-1791.

Kim J., Cho Y.A., Kim D.H., Lee B.H., Hwang D.Y., Jeong J., Lee H.J., Ly Matsuo K., Tajima K., Ahn Y.O. (2012). Dietary intake of folate and alcohol, MTHFR C677T polymorphism, and colorectal cancer risk in Korea. American Journal of Clinical Nutrition, 95 (2), 405-412.

Kim Y.I., Christman J.K., Fleet J.C., Cravo M.L., Salomon R.N., Smith D., Ordovas J., Selhub J., Mason J.B. (1995). Moderate folate deficiency does not cause global hypomethylation of hepatic and colonic DNA or c-mycspecific hypomethylation of colonic DNA in rats. American Journal of Clinical Nutrition, 61, 1083-1090.

Kim Y.I., Pogribny I.P., Salomon R.N., Choi S.W., Smith D.E., James S.J., Mason J.B. (1996a). Exon-specific DNA hypomethylation of the p53 gene of rat colon induced by dimethylhydrazine. Modulation by dietary folate. American Journal of Pathology, 149, 1129-1137.

Kim Y.I., Salomon R.N., Graeme-Cook F., Choi S.W., Smith D.E., Dallal G.E., Mason J.B. (1996b). Dietary folate protects against the development of macroscopic colonic neoplasia in a dose responsive manner in rats. International Journal of Gastroenterology and Hepatology, 39, 732-740.

Kim Y.I., Pogribny I.P., Basnakian A.G., Miller J.W., James S.J., Mason J.B. (1997). Folate deficiency in rats induces DNA strand breaks and hypomethylation within the p53 tumor suppressor gene. American Journal of Clinical Nutrition, 65 (1), 46-52.

Kim Y.I. (1999a). Folate and cancer prevention: a new medical application of folate, beyond hyperhomocysteinemia and neural tube defects. Nutrition Reviews, 57, 314-321.

Kim Y.I. (1999b). Folate and carcinogenesis: evidence, mechanisms, and implications. Journal of Nutrition and Biochemistry, 10 (2), 66-88. 
Kim Y.I., Shirwadkar S., Choi S.W., Puchyr M., Wang Y., Mason J.B. (2000). Effects of dietary folate on DNA strand breaks within mutation-prone exons of the $p 53$ gene in rat colon. Gastroenterology, 119, 151-161.

Kim Y.I., Baik H.W., Fawaz K., Knox T., Lee Y.M., Norton R., Libby E., Mason J.B. (2001). Effects of folate supplementation on two provisional molecular markers of colon cancer: a prospective, randomized trial. American Journal of Gastroenterology, 96, 184-195.

Kim Y.I. (2003). Role of folate in colon cancer development and progression. Journal of Nutrition, 133, 3731-3739.

Kim Y.I. (2004). Folate, Colorectal Carcinogenesis, and DNA Methylation: Lessons From Animal Studies. Environmental and Molecular Mutagenesis, 44, 10-25.

Kimoto T., Suzuki K., Kobayashi X.M., Dobrovolsky V.N., Heflich R.H., Miura D., Kasahara Y. (2011b). Manifestation of mutant bone marrow erythroids and peripheral blood erythrocytes in mice treated with $\mathrm{N}$-ethyl-N-nitrosourea: Direct sequencing of Pig-a cDNA from bone marrow cells negative for GPIanchored protein expression. Mutation Research, 723, 36-42.

Kinzler K.W., Vogelstein B. (1996). Lessons from hereditary colorectal cancer. Cell, 18 (2), 159-170.

Knock E., Deng L., Wu Q., Lecrerc D., Wang X.L., Rosen R. (2006). Low dietary folate initiates intestilan tumors in mice, with alteres expression of G2$\mathrm{M}$ checkpoint regulators polo-like kinase 1 and cell division cycle 25c. Cancer research, 66, 10349-10356.

Knock E., Deng L., Wu Q., Lawrance A.K., Wang X.L., Rosen R. (2008). Strain differences in mice highlight the role of DNA damage in neoplasia induced by low dietary folate. Journal of Nutrition, 138, 653-658.

Kovacheva V.P., Mellott T.J., Davison J.M., Wagner N., Lopez-Coviella I., Schnitzler A.C., Blusztajn J.K. (2007). Gestational choline deficiency causes global and lgf2 gene DNA hypermethylation by up-regulation of Dnmt1 expression. Journal of Biology and Chemistry, 282 (43), 31777-31788.

Koyama S. (1960). Studies on Howell-Jolly body. Acta of Haematogy Japan, 23, 20-25.

Kraunz K.S., Hsiung D., McClean M.D., Liu M., Osanyingbemi J., Nelson H.H., Kelsey K.T. (2006). Dietary folate is associated with p16INK4A methylation in head and neck squamous cell carcinoma. International Journal of Cancer, 119, 1553-1557. 
Krebs-Smith S.M., Cook A., Subar A.F., Cleveland L., Friday J. (1995). US adults fruit and vegetable intakes, 1989-1991: a revised baseline for the healthy people 2000 objective. American Journal of Public Health, 85, 16231629.

Krebs-Smith S.M., Cook A., Subar A.F., Cleveland L., Friday J., Kahle L.L. (1996). Fruit and vegetable intakes of children and adolescents in the United States, Archives of Pediatric and Adolescent Medicine, 150, 81-86.

Krokan H.E., Drablos F., Slupphaug G. (2002). Uracil in DNA-occurrence, consequences and repair. Oncogene, 21, 8935-8948.

Krumdieck C.L. (1991). In: Vitamins and Cancer Prevention. Localised folate deficiency and cancer. Laidlaw S.A., Swendseid M.E. (eds). Wiley, New York, pp. 39-49.

Kuo K.C., McCune R.A., Gehrke C.W. (1980). Quantitative reversed-phase high performance liquid chromatographic determination of major and modified deoxyrobonucleosides in DNA. Nucleic Acids Research, 8, 4763-76.

Larsson S.C., Giovannucci E., Wolk A. (2006). Folate intake, MTHFR polymorphisms, and risk of esophageal, gastric, and pancreatic cancer: a mata-analysis. Gastroenterology, 131 (4), 1271-1283.

Lawrance A.K., Deng L., Brody L.C., Finnell R.H., Shane B., Rozen R. (2007). Genetic and nutritional deficiencies in folate metabolism influence tumorigenicity in $\mathrm{Apc}^{\mathrm{min} /+}$ mice. Journal of Nutritional Biochemistry, 18, 305312.

Lawrance A.K., Deng L., Rozen R. (2009). Methylenetetrahydrofolate reductase deficiency and low dietary folate reduce tumorigenesis in $A p c^{\mathrm{min} /+}$ mice. International Journal of Gastroenterology and Hepatology, 58, 805-811.

Le Leu R.K., Young G.P., McIntosh G.H. (2000). Folate deficiency diminishes the occurrence of aberrant crypt foci in the rat colon but does not alter global DNA methylation status. International Journal of Gastroenterology and Hepatology, 15, 1158-1164.

Le Marchand L., Yoshizawa C.N., Kolonel L.N., Hankin J.H., Goodman M.T. (1989). Vegetable consumption and lung cancer risk: a population based case-control study in Hawaii. Journal of National Cancer Institute, 81 (15), 1158-1164.

Lee J.E., Wei E.K., Fuchs C.S., Hunter D.J., Lee I.M., Selhub J., Stampfer M.J., Willett W.C., Ma J., Giovannucci E. (2012). Plasma folate, methylene tetrahydropholate reductase (MTHFR), and colorectal cancer risk in three large nested case-control studies. Cancer Causes and Control, 4, 537-545. 
Lessin L.S., Bessis M. (1972). In: Hematology. Morphology of the erythron. Williams W.J., Beutler E., Erslev A.J., Rundles R.W. (eds). McGraw-Hill, New York, pp. 62-93.

Li E., Bestor T.H., Jaenisch R. (1992). Targeted mutation of the DNA methyltransferase gene results in embryonic lethality. Cell, 69, 915-926.

Li J.Y., Lees-Murdock D.J., Xu G.L., Walsh C.P. (2004). Timing of establishment of paternal methylation imprints in the mouse. Genomics, 84, 952-960.

Li Y., Lalancette C., Miller D., Krawetz S.A. (2008). Characterization of nucleohistone and nucleoprotamine components in the mature human sperm nucleus. Asian Journal of Andrology, 10, 535-541.

Li Y.Q., Zhou P.Z., Zheng X.D., Walsh C.P., Xu G.L. (2007). Association of Dnmt3a and thymine DNA glycosylase links DNA methylation with baseexcision repair. Nucleic Acids Research, 35, 390-400.

Li Z., Yang J., Huang H. (2006). Oxidative stress induces H2AX phosphorylation in human spermatozoa. FEBS Letters, 580, 6161-6168

Linhart H.G., Troen A., Bell G.W., Cantu E., Chao W.H., Moran E., Steine E., He T., Jaenisch R. (2009). Folate deficiency induces genomic uracil misincorporation and hypomethylation but does not increase DNA point mutations. Gastroenterology, 136, 227-235.

Lister R., Pelizzola M., Dowen R.H., Hawking R.D., Hon G., Tonti-Filippini J., Nery J.R., Lee L., Ye Z., Ngo Q.M., Edsall L., Antosiewicz-Bourget J., Stewart R., Ruotti V., Millar A.H., Thomson J.A., Ren B., Ecker J.R. (2009). Human DNA methylomes at base resolution show widespread epigenomic differences. Nature, 19 (462), 315-322.

Liu H.L., Zhou Y., Boggs S.E., Belinsky S.A., Liu J. (2007). Cigarette smoke induces demethylation of prometastatic oncogene synuclein-gamma in lung cancer cells by downregulation of DNMT3B. Oncogene, 26, 5900-5910.

Loeb L.A., Loeb K.R., Anderson J.P. (2003). Multiple mutations and cancer. Proceedings of the National Academy of Science USA, 100, 776-871.

Logan R.F., Grainge M.J., Shepherd V.C., Armitage N.C., Muir K.R., ukCAP trial Group. (2008). Aspirin and folic acid for the prevention of recurrent colorectal adenomas. Gastroenterology, 134 (1), 29-38.

Long C., He J., Liu X., Chen X., Gao R., Wang Y., Ding Y. (2012) Dietary folate deficiency in pseudopregnant mice has no effect on homebox A10 promoter methylation or expression. Reproductive Sciences, 1, 1-8.

Luongo C., Moser A.R., Gledhill S., Dove W.F. (1994). Loss of Apc in intestinal adenomas from Min mice. Cancer Research, 54, 5947-5952. 
Ly A., Lee H., Chen J., Sie K.K.Y., Renlund R., Medline A., Sohn K.J., Croxford R., Thompson L.U., Kim Y.I. (2011). Effect of maternal and post weaning folic acid supplementation on mammary tumor risk in the offspring. Cancer Research, 71 (3), 988-997.

McCann J.C., Hudes M., Ames B.N. (2006). An overview of evidence for a causal relationship between dietary availability of choline during development and cognitive function in offspring. Neuroscience and Biobehavioral Reviews, 30 (5), 696-712.

MacGregor J.T., Wehr C.M., Hiatt R.A., Peteres B., Tucker J.D., Langlois R.G., Jacob R.A., Jensen R.H., Yager J.W., Shigenaga M.K., Frei B., Eynon B.P., Ames B.N. (1997). Spontaneous genetic damage in man: Evaluation of inter-individual variability, relationship among markers of damage, and influence of nutritional status. Mutation Research, 377, 125-135.

Mayne S.T., Risch H.A., Dubrow R., Chow W.H., Gammon M.D., Vaughan T.L., Farrow D.C., Schoenberg J.B., Stanford J.L., Ahsan H., West B., Rotterdam H., Blot W.J., Fraumeni J.F. (2001). Nutrient intake and risk of subtypes of esophageal and gastric cancer. Cancer Epidemiology Biomarkers and Prevention, 10, 1055-1062.

McKay J.A., Williams E.A., Mathers J.C. (2008). Sex-specific modulation of tumorigenesis by folic acid supply in the $A p c$ mouse during early neonatal life. British Journal for Nutrition, 99, 550-558.

McKay J.A., Waltham K.J., Williams E.A., Mathers J.C. (2011a). Folate depletion during pregnancy and lactation reduces genomic DNA methylation in murine adult offspring. Genes and Nutrition, 6, 189-196.

McKay J.A., Williams E.A., Mathers J.C. (2011b). Effect of maternal and post weaning folate supply on gene-specific DNA methylation in the small intestine of weaning and adult $A p c^{+/ M i n}$ and wild type mice. Frontiers in Genetics, 2, 23.

McKay J.A., Xie L., Harris S., Wong Y.K., Ford D., Mathers J.C. (2011c). Blood as a surrogate marker for tissue-specific DNA methylation and changes due to folate depletion in post-partum female mice. Molecular Nutrition and Food Research, 55, 1026-1035.

Meck W.H., Williams C.L. (2003). Metabolic imprinting of choline by its availability during gestation: Implications for memory and attentional processing across the lifespan. Neuroscience and Biobehavioral Reviews, 27 (4), 385-399.

Meck W.H., Williams C.L., Cermak J.M., Blusztajn J.K. (2008). Developmental periods of choline sensitivity provide an ontogenetic mechanism for regulating memory capacity and age-related dementia. Frontiers of Integrative Neuroscience, 1, 1-11. 
Mehedint M.G., Craciunescu C.N., Zeisel S.H. (2010). Maternal dietary choline deficiency alters angiogenesis in fetal mouse hippocampus. Proceedings of the National Academy of Science USA, 107 (29), 1283412839.

Mellott T.J., Williams C.L., Meck W.H., Blusztajn J.K. (2007). Prenatal choline deficiency increases choline transporter expression in the septum and hippocampus during postnatal development and in adulthood in rats. Federation of American Societies of Experimental Biology, 18, 1-11.

Metivier R., Gallais R., Tiffoche C., Le Péron C., Jurkowska R.Z. (2008). Cyclical DNA methylation of a transcriptionally active promoter. Nature, $\mathbf{4 5 2}$, $45-50$.

Meuth M. (1998). The molecular basis of mutation induced deoxyribonucleoside triphosphate pool imbalances in mammalian cells. Experimental Cell Research, 181, 305-316.

Miller A.L. (2003). The methionine-homocysteine cycle and its effects on cognitive diseases - homocysteine and cognitive. Alternative Medicine Reviews, 8, 7-19.

Miller C., Sweatt J. (2007). Covalent modification of DNA regulates memory formation. Neuron, 53 (6), 857-869.

Miller D., Brinkworth M., lles D. (2010). Paternal DNA packaging in spermatozoa: more than the sum of its parts? DNA, histones, protamines and epigenetics. Reproduction, 139, 287-301.

Mills P.K., Beeson W.L., Phillips R.L., Fraser G.E. (1994). Cancer incidence among California seventh-day adventists. American Journal of Clinical Nutrition, 59, 1136-1142.

Miura D., Dobrovolsky V.N, Kasahara Y., Katsuura Y., Heflich R.H. (2008). Development of an In Vivo Gene Mutation Assay Using the Endogenous PigA Gene: I. Flow Cytometric Detection of CD59-Negative Peripheral Red Blood Cells and CD48-Negative Spleen T-Cells From the Rat. Environmental and Molecular Mutagenesis, 49, 614-621.

Morgan H.D., Dean W., Coker H.A., Reik W., Petersen-Mahrt S.K. (2004). Activation-induced cytidine deaminase deaminates 5-methylcytosine in DNA and is expressed in pluripotent tissues: implications for epigenetic reprogramming. Journal of Biology and Chemistry, 279, 52353-52360.

Morgan H.D., Santos F., Green K., Dean W., Reik W. (2005). Epigenetic reprogramming in mammals. Human Molecular Genetics, 14, 47-58. 
Myant K., Sansom O.J. (2011). Wnt/Myc interactions in intestinal cancer: Partners in crime. Experimental Cell Research, 317, 2725-2731.

Nakagawa T., Kanai Y., Ushijima S., Kitamura T., Kakizoe T., Hirohashi S. (2005). DNA hypomethylation on pericentromeric satellite regions significantly correlates with loss of hetero- zygosity on chromosome 9 in urothelial carcinomas. Journal of Urology, 173, 243-246.

Nestle M. (2002). Food Politics. Berkeley: University of California. ISBN 9790520224659.

Newmark H.L., Yang K., Lipkin M., Kopelovich L., Liu Y., Fan K., Shinozaki $\mathrm{H}$. (2001). Awestern-style diet induces benign and malignant neoplasms in the colon of normal C57BI/6 mice. Carcinogenesis, 22, 1871-1875.

Newmark H.L., Yang K., Kurihara N., Fan K., Augenlicht L.H., Lipkin M. (2009). Western-style diet-induced colonic tumors and their modulation by calcium and vitamin D in C57BI/6 mice: a preclinical model for human sporadic colon cancer. Carcinogenesis, 30, 88-92.

Niculescu M.D., Zeisel S.H. (2002). Diet, methyl donors and DNA methylation: Interactions between dietary folate, methionine and choline. Journal of Nutrition, 132, 2333-2335.

Niculescu M.D., Craciunescu C.N., Zeisel S.H. (2006). Dietary choline deficiency alters global and gene-specific DNA methylation in the developing hippocampus of mouse fetal brains. Federation of American Societies of Experimental Biology, 20 (1) 43-49.

Nilsen H., Rosewell I., Robins P., Skjelbred C.F., Andersen S., Slupphaug G., Daly G., Krokan H.E., Lindahl T., Barnes D.E. (2000). Uracil-DNA glycosylase (UNG)-deficient mice reveal a primary role of the enzyme during DNA replication. Molecular Cell, 5, 1059-1065.

Nilsson E.E., Anway M.D., Stanfield J., Skinner M.K. (2008). Transgenerational epigenetic effects of the endocrine disruptor vinclozolin on pregnancies and female adult onset disease. Reproduction, 135, 713-721.

Noonan K.E., Beck C., Holzmayer T.A., Chin J.E., Wunder J.S., Andrulis I.L., Gazdar A.F., Willman C.L., Griffith B., Von Hoff D.D. (1990). Quantitative analysis of MDR1 (multidrug resistance) gene expression in human tumors by polymerase chain reaction. Proceedings of the National Academy of Science, 87, 7160-7164.

Obata Y., Kono T. (2002). Maternal primary imprinting is established at a specific time for each gene throughout oocyte growth. Journal of Biological Chemistry, 277, 5285-5289. 
Ogino S., Nosho K., Kirkner G.J., Kawasaki T., Chan A.T., Schernhammer E.S., Giovannucci E.L., Fuchs C.S. (2008). A cohort study of tumoral LINE-1 hypomethylation and prognosis in colon cancer. Journal of National Cancer Institute, 100, 1734-1738.

Ohtani S., Unno A., Ushiyama A., Kimoto T., Miura D., Kunugita N. (2012). The In Vivo Pig-a Gene Mutation Assay is Useful for Evaluating the Genotoxicity of lonizing Radiation in Mice. Environmental and Molecular Mutagenesis, 53, 579-588.

Okuwaki M., Verreault A. (2004). Maintenance DNA Methylation of nucleosome core particles. Journal of Biological Chemistry, 279 (4), 29042912.

Oommen A.M., Griffin J.B., Sarath G., Zempleni J. (2005). Roles of nutrients in epigenetic events. Journal of Nutritional Biochemistry, 16, 74-77.

Padmanabhan S., Tripathi D.N., Vikram A., Ramarao P., Jena G.B. (2008). Cytotoxic and genotoxic effects of methotrexate in germ cells of male Swiss mice. Mutation Research, 655, 59-67.

Padmanabhan S., Tripathi D.N., Vikram A., Ramarao P., Jena G.B. (2009). Methotrexate-induced cytotoxicity and genotoxicity in germ cells of mice: Intervention of folic and folinic acid. Mutation Research, 673, 43-52.

Polakis P. (2000). Wnt signaling and cancer. Genes and Development, 14, 1837-1851.

Pelucchi C., Galeone C., Talamini R., Negri E., Parpinel M., Franceschi S., Montella M., La Vecchia C. (2005). Dietary folate and risk of prostate cancer in Italy. Cancer Epidemiology Biomarkers and Prevention, 14, 944-948.

Petronis A. (2010). Epigenetics as a unifying principle in the aetiology of complex traits and diseases. Nature, 465 (10), 721-727.

Phillips T. (2008). The role of methylation in gene expression. Nature Education, 1, 1.

Phonethepswath S., Bryce S.M., Bemis J.C., Dertinger S.D. (2008). Erythrocyte-based Pig-a gene mutation assay: Demonstration of crossspecies potential. Mutation Research, 657,122-126.

Pittoggi C., Renzi L., Zaccagnini G., Cimini D., Degrassi F., Giordano R., Magnano A.R., Lorenzini R., Lavia P., Spadafora C. (1999). A fraction of mouse sperm chromatin is organized in nucleosomal hypersensitive domains enriched in retroposon DNA. Journal of Cell Science, 112, 3537-3548. 
Piyathilake C.J., Macaluso M., Hine R.J., Vinter D.W., Richards E.W., Krumdieck C.L. (1995). Cigarette smoking, intracellular vitamin deficiency and occurrence of micronuclei in epithelial cells of the buccal mucosa. Cancer Epidemiology Biomarkers \& Prevention, 4, 751-758.

Piyathilake C.J., Macaluso M., Celedonio J.E., Badiga S., Bell W.C., Grizzle W.E. (2010). Mandatory fortification with folic acid in the United States appears to have 968 adverse effects on histone methylation in women with pre-cancer but not in women free of pre-cancer. International Journal for Women Health, 1, 131-137.

Pogribny I.P., Basnakian A.G., Miller B.J., Lopatina N.G., Poirier L.A., James S.J. (1995). Breaks in genomic DNA and within the p53 gene are associated with hypomethylation in livers of folate/lethyl-deficient rats. Journal of Cancer Research, 55, 1931-1940.

Pogribny I.P., Muskhelishvili L., Miller J.B., James S.J. (1997). Presence and consequence of uracil in pre-neoplastic DNA from folate/methyl-deficient rats. Carcinogenesis, 18, 2071-2076.

Pogribny I.P., Ross S.A., Wise C., Progribna M., Jones E.A., Tryndyak V.P., James S.J., Dragan Y.P., Poirer L.A (2006). Irreversible global DNA hypomethylation as a key step in hepatocarcinogenesis induced by dietary methyl deficiency. Mutation Research, 593, 80-87.

Pogribny I.P., Rusyn I., Beland F.A. (2008). Epigenetic aspects of genotoxic and non-genotoxic hepatocarcinogenesis; studies in rodents. Environmental Molecular Mutagenesis, 49, 9-15.

Pogribny I.P., Beland F.A. (2009). DNA hypomethylation in the origin and pathogenesis of human diseases. Cellular and Molecular Life Sciences, 9, 13.

Pogribny I.P., Shpylevaa S.I., Muskhelishvilic B.L., Bagnyukovaa T.V., James S.J., Beland F.A. (2009a). Role of DNA damage and alterations in cytosine DNA methylation in rat liver carcinogenesis induced by a methyl-deficient diet. Mutation Research, 669, 56-62.

Pogribny I.P., Tryndyak V.P., Bagnyukova T.V., Melnyk S., Montgomery B., Ross S.A., Latendresse J.R., Rusyn I., Beland F.A. (2009b). Hepatic epigenetic phenotype predetermines individual susceptibility to hepatic steatosis in mice fed a lipogenic methyl-deficient diet. Journal of Hepatology, 1, 1-11.

Pogribny I.P., James S.J., Beland F.A. (2012). Molecular alterations in hepatocarcinogenesis induced by dietary methyl deficiency. Molecular Nutrition and Food Research, 55, 116-125. 
Pufulete M., Ghnaniem R.A.I., Khushal A., Appleby P., Harris N., Gout S., Emery P.W., Sanders T.A. (2005a). Effect of folic acid supplementation on genomic DNA methylation in patients with colorectal adenoma. International Journal of Gastroenterology and Hepatology, 54 (5), 648-653.

Pufulete M., Ghnaniem R.A.I., Rennie J.A., Appleby P., Harris N., Gout S., Emery P.W., Sanders T.A. (2005b). Influence of folate status on genomic DNA methylation in colonic mucosa of subjects without colorectal adenoma or cancer. British Journal of Cancer, 92 (5), 838-842.

Pyapali G.K., Turner D.A., Williams C.L., Meck W.H., Swartzwelder H.S. (1998). Prenatal dietary choline supplementation decreases the threshold for induction of long-term potentiation in young adult rats. Journal of Neurophysiology, 79, 1790-1796.

Quinlivan E.P., Gregory J.F. (2003). Effect of food fortification on folic acid intake in the United States. American Journal of Clinical Nutrition, 77, 221225.

Rampersaud G.C., Kauwell G.P.A., Hutson A.D., Cerda J.J., Bailey L.B. (2000). Genomic DNA methylation decreases in response to moderate folate depletion in elderly women. American Journal of Clinical Nutrition, 72 (4), 998-1003.

Rauch T.A., Zhong X., Wu X., Wang M., Kernstine K.H., Wang Z., Riggs A.D., Pfeifer G.P. (2008). High-resolution mapping of DNA hypermethylation and hypomethylation in lung cancer. Proceedings of the National Academy of Science USA, 105, 252-257.

Reik W. (2007). Stability and flexibility of epigenetic gene regulation in mammalian development. Nature, 447, 425-432.

Reik W., Dean W. (2002). Epigenetic reprogramming: Back to the beginning. Nature, 420, 127.

Rideout, W.M., Eggan K., Jaenisch R. (2001). Nuclear cloning and epigenetic reprogramming of the genome. Nature, 403, 501-502.

Roach J.C., Glusman G., Smit A.F., Huff C.D., Hubley R., Shannon P.T., Rowen L., Pant K.P., Goodman N., Bamshad M., Shendure J., Drmanac R., Jorde L.B., Hood L., Galas D.J. (2010). Analysis of genetic inheritance in a family quartet by whole-genome sequencing. Science, 328, 636-639.

Robert M.F., Morin S., Beaulieu N., Gauthier F., Chute I.C., Barsalou A., MacLeod A.R. (2002). DNMT1 is required to maintain CpG methylation and aberrant gene silencing in human cancer cells. Nature Genetics, 33, 61-65.

Robertson K.D (2005). DNA methylation and human disease. Nature Reviews Genetics, 6, 597-610. 
Robertson K.D., Wolffe A.P. (2000). DNA methylation in health and disease. National Reviews Genetics, 1 (1), 11-19.

Rollins R.A., Haghighi F., Edwards J.R., Das R., Zhang M.Q., Ju J., Bestor T.H. (2006). Large-scale structure of genomic methylation patterns. Genome Research, 16, 157-163.

Saiki R.K., Gelfand D.H., Stoffel S., Scharf S.J., Higuchi R., Horn G.T. Mullis K.B., Erlich H.A. (1988). Primer-directed enzymatic amplification of DNA with thermostable DNA polymerase. Science, 239, 487-91.

Sambrook J., Russell D. W. (2001). In: Molecular cloning: A laboratory manual. Cold Spring Harbour Laboratory, New York, pp. 818-830.

Sandeman F. (2000). Minerals: Benefits and food sources of dietary minerals. http://www.recipes4us.co.uk/Minerals.htm (assessed 17-04-12).

Sandstrom N.J., Loy R., Williams C.L. (2002). Prenatal choline supplementation increases NGF levels in the hippocampus and frontal cortex of young and adult rats. Brain Research, 947, 9-16.

Santos F., Hendrich B., Reik W., Dean W. (2002). Dynamic reprogramming of DNA methylation in the early mouse embryo. Developmental Biology, 214, $172-182$.

Santos F., Dean W. (2004). Epigenetic reprogramming during early development in mammals. Journal of Reproduction and Fertility, 127, 14701626.

Scholl T.O., Johnson W.G. (2000). Folic acid: Influence on the outcome of pregnancy. American Journal of Clinical Nutrition, 71, 129-303.

Searle A.G. (1974). Mutation induction in mice. Advances in Radiation Biology, 4, 131-207.

Seitz H.K., Stickel F. (2007). Molecular mechanisms of alcohol-mediated carcinogenesis. Nature Reviews Cancer, 7, 599-612.

Shanks M., Riou L., Fouchet P., Dubrova Y.E. (2008). Stage-specificity of spontaneous mutation at a tandem repeat DNA locus in the mouse germline. Mutation Research, 641, 58-60.

Shanmugham J.R., Zavras A.I., Rosner B., Giovannucci E. (2010). Alcoholfolate interactions in women's oral cancer risk: A prospective cohort study. Cancer Epidimiological Biomarkers and Prevention, 19 (10), 2516-2524.

Shaw G.M., Carmichael S.L., Yang W., Selvin S., Schaffer D.M. (2004). Periconceptional dietary intake of choline and betaine and neural tube defects in offspring. American Journal of Epidemiology, 160, 102-109. 
Shelnutt K.P., Kauwell G.P.A., Gregory J.F., Maneval D.R., Quinlivan E.P., Theriaque D.W., Henderson G.N., Bailey L.B. (2004). Methylenetetrahydrofolate reductase $677 \mathrm{C} \rightarrow T$ polymorphism affects DNA methylation in response to controlled folate intake in young women. Journal of Nutritional Biochemistry, 15 (9), 554-560.

Shi Y., Lan F., Matson C., Mulligan P., Whetstine J.R. (2004). Histone demethylation mediated by the nuclear amine oxidase homolog LSD1. Cell, 119, 941-953.

Sibani S., Melnyk S., Pogribny I.P., Wang W., Tim F.H., Deng L., Trasler J., James S.J., Rozen R. (2002). Studies of methionine cycle intermediates (SAM, SAH), DNA methylation and the impact of folate deficiency on tumour numbers in Min mice. Carcinogenesis, 23 (1), 61-65.

Sie K.K.Y., Medline A., van Weel J., Sohn K.J., Choi S.W., Croxford R., Kim Y.I. (2011). Effect of maternal and post-weaning folic acid supplementation on colorectal cancer risk in the offspring. International Journal of Gastroenterology and Hepatology, 60, 1687-1694.

Sieber O.M., Lipton L., Crabtree M., Heinimann K., Fidalgo P., Phillips R.K.S., Bisgaard M.L., Orntoft T.F., Aaltonen L.A., Hodgson S.V., Thomas H.J.W., Tomlinson I.P.M. (2003). Multiple colorectal adenomas, classic adenomatous polyposis, and germ-line mutations in $\mathrm{MYH}$. New England Journal of Medicine, 348, 791-799.

Singer T.M., Yauk C.L. (2010). Germ cell mutagens: Risk assessment challenges in the $21^{\text {st }}$ century. Environmental and Molecular Mutagenesis, $51,991-928$.

Skibola C.F., Smith M.T., Kane E., Roman E., Rollinson S., Cartwright R.A., Morgan G. (1999). Polymorphisms in the methylenetetrahydrofolate reductase gene are associated with susceptibility to acute leukemia in adults. Proceedings of the National Academy of Science USA, 96, 12810-12815.

Skinner H.G., Michaud D.S., Giovannucci E.L., Rimm E.B., Stampfer M.J., Willett W.C., Colditz G.A., Fuchs C.S. (2004). A prospective study of folate intake and the risk of pancreatic cancer in men and women. American Journal of Epidemiology, 160 (3), 248-158.

Smith D.F., MacGregor J.T., Hiatt R.A., Hooper N.K., Wehr C.M., Peters B., Goldman L.R., Yuan L.A., Smith P.A., Becker C.E. (1990). Micronucleated erythrocytes as an index of cytogenetic damage in humans: Demographic and dietary factors associated with micronucleated erythrocytes in splenectomised subjects. Cancer Research, 50, 5049-5054.

Sohn K.J., Stempak J.M., Reid S., Shirwadkar S., Mason J.B., Kim Y.I. (2003). The effect of dietary folate on genomic and p53-specific DNA methylation in rat colon. Carcinogenesis, 24 (1), 81-90. 
Solov'eva L., Svetlova M., Bodinski D., Zalensky A.O. (2004). Nature of telomere dimers and chromosome looping in human spermatozoa. Chromosome Research, 12, 817-823.

Somers C.M., Yauk C.L., White P.A., Parfett C.L.J., Quinn, J.S. (2002). Air pollution induces heritable DNA mutations. Proceedings of the National Academy of Sciences USA, 99, 15904-15907.

Somers C.M. (2006). Expanded simple tandem repeat (ESTR) mutation induction in the male germline: lessons learned from lab mice. Mutation Research, 598 (1-2), 35-49.

Song J., Medline A., Mason J.B., Gallinger S., Kim Y.I. (2000a). Effects of dietary folate on intestinal tumorigenesis in the $A p c^{M i n}$ mouse. Cancer Research, 60, 5434-5440.

Song J., Sohn K.J., Medline A., Ash C., Gallinger S., Kim Y.I. (2000b). Chemopreventive effects of dietary folate on intestinal polyps in $A p c^{+/ M s h 2}$ mice. Cancer Research, 60, 3191-3199.

Soon L.L., Ausio J., Breed W.G., Power J.H., Muller S. (1997). Isolation of histones and related chromatin structures from spermatozoa nuclei of a dasyurid marsupial, Sminthopsis crassicaudata. Journal of Experimental Zoology, 278, 322-332.

Starlard-Davenport A., Tryndyak V., Kosyk O., Ross S.R., Rusyn I., Beland F.A., Pogribny I.P. (2011). Dietary methyl deficiency, microRNA expression and susceptibility to liver carcinogenesis. Journal of Nutrigenetics and Nutrigenomics, 3, 259-266.

Steinmetz K.A., Potter J.D. (1996). Vegetables, fruit and cancer prevention: a review. Journal of American Diet Association, 96, 1027-1039.

Stidley C.A., Picchi M.A., Leng S., Willink R., Crowell R.E., Flores K.G., Kang H., Byers T., Gilliland F.D., Belinsky S.A. (2010). Multivitamins, folate, and green vegetables protect against gene promoter methylation in the aerodigestive tract of smokers. Cancer Research, 70 (2), 568-574.

Stolzenberg-Solomon, R.Z., Albanes D., Nieto F.J., Hartman T.J., Tangrea J.A., Rautalahti M., Sehlub J., Virtamo J., Taylor P.R. (1999). Pancreatic cancer risk and nutrition-related methyl-group availability indicators in male smokers. Journal of National Cancer Institute, 91, 535-541.

Stolzenberg-Solomon, R., Pietinen P., Barrett M.J., Taylor P.R., Virtamo J., Albanes D. (2001). Dietary and other methyl group availability factors and pancreatic cancer risk in a cohort of male smokers. American Journal of Epidemiology, 153, 680-687. 
Stolzenberg-Solomon R., Chang S.C., Leitzmann M.F., Johnson C., Buys S.S., Hoover R.N., Ziegler R.G. (2006). Folate intake, alcohol use, and postmenopausal breast cancer risk in the prostate, lung, colorectal and ovarian cancer screening trial. American Journal of Clinical Nutrition, 83 (4), 895-904.

Strachan T., Read A.P. (1999). In: Human molecular genetics: Instability of the human genome: mutation and DNA repair. Wiley-Liss, New York, pp. 560.

Streffer C., Shore R., Konermann G., Meadows A., Uma Devi P., Preston Withers J., Holm L.E., Stather J., Mabuchi K. (2003). Biological effects after prenatal irradiation (embryo and fetus). A report of the International Commission on Radiological Protection.

Annals of the ICRP, 33 (1-2), 5-206.

Su L.J. (2012). In: Cancer Epigenetics: Methods of Molecular Biology. Diet, epigenetics and cancer. Dumitrescu R.G., Verma M. (eds). Springer-Verlang, New York, pp. 377-393.

Suzuki M.M., Bird A. (2008). DNA methylation landscapes: Provocative insights from epigenomics. Nature Reviews Genetics, 9, 465-476.

Swayne B.G., Behan N.A., Williams A.,Stover P.J., Yauk C.L, MacFarlane A.J. (2012a). Supplemental dietary folic acid has no effect on chromosome damage in erythrocyte progenitor cells of mice. Journal of Nutrition, 142, 813817.

Swayne B.G., Kawata A., Behan N.A., Williams A., Wade M.G., MacFarlane A.J., Yauk C.L. (2012b). Investigating the effects of dietary folic acid on sperm count, DNA damage and mutation in Balb/c mice, Mutation Research, Fundamental and Molecular Mechanisms of Mutagenesis, 737, 1-7.

Tawn E.J., Whitehouse C.A., Winther J.F., Curwen G.B., Rees G.S., Stovall M., Olsen J.H., Guldberg P., Rechnitzer C., Schrøder H., Boice J.D. (2005). Chromosome analysis in childhood cancer survivors and their offspring--no evidence for radiotherapy-induced persistent genomic instability. Mutation Research, 583, 198-206.

Teh A.H., Symonds E., Bull C., Clifton P., Fenech M. (2012). The influence of folate and methionine on intestinal tumor development in the $A p c^{\mathrm{Min} /+}$ mouse model. Mutation Research, 75 (1), 64-75.

Tegelenbosch R.A.J., De Rooij D.G. (1993). A quantitative study of spermatogonial multiplication and stem cell renewal in the $\mathrm{C} 3 \mathrm{H} / 101 \mathrm{~F}_{1}$ hybrid mice. Mutation Research, 290, 193-200.

The International Chernobyl Project. (1991). Technical Report. IAEA, Vienna. 
Thompson J.R., Gerald P.F., Willoughby M.L.N., Armstrong B.K. (2001). Maternal folate supplementation in pregnancy and protection against acute lymphoblastic leukaemia in childhood: A case-control study. The Lancet, 358 (9297), 1935-1940.

Trasler J., Deng L., Melnyk S., Pogribny I., Hiou-Tim F., Sibani S., Oakes C., Li E., James S.J., Rozen R. (2003). Impact of Dnmt1 deficiency, with and without low folate diets, on tumor numbers and DNA methylation in Min mice. Carcinogenesis, 24, 39-45.

Trewick S.C., Henshaw T.F., Hausinger R.P., Lindahl T., Sedgwick B. (2002). Oxidative demethylation by Escherichia coli AlkB directly reverts DNA base damage. Nature, 419, 174-178.

Tryndyak V.P., Ross S.A., Beland F.A., Pogribny I.P. (2009). Downregulation of the microRNAs miR-34a, miR-127, and miR-200b in rat liver during hepatocarcinogenesis induced by a methyl-deficient diet. Molecular Carcinogenesis, 48, 479-487.

Tsukada Y., Fang J., Erdjument-Bromage H., Warren M.E., Borchers C.H. (2006). Histone demethylation by a family of JmjC domain-containing proteins. Nature, 439, 811-816.

Tucker K.L. (2001). Methylated cytosine and the brain: A new base for neuroscience. Neuron, 30 (3), 649-652.

Tucker J.M., Davis C., Kitchens M.E., Bunni M.A., Priest D.G., Spencer H.T., Berger F.G. (2002). Response to 5-fluorouracil chemotherapy is modified by dietary folicacid deficiency in $\mathrm{Apc}^{\mathrm{Min} /+}$ mice. Cancer Letters, 187, 53-162.

Ulrey C.L., Liu L., Andrews L.G., Tollefsbol T.O. (2005). The impact of metabolism on DNA methylation. Human Molecular Genetics, 14, 139-147.

Ulrich C.M., Kampman E., Bigler J., Schwartz S.M., Chen C., Bostick R., Fosdick L., Beresford S.A.A., Yasui Y., Potter J.D. (2000). Lack of association between the C677T MTHFR polymorphism and colorectal hyperplasic polyps. Cancer Epidemiology Biomarkers and Prevention, 9, 427-433.

Valinluck V., Tsai H.H., Rogstad D.K., Burdzy A., Bird A., Sowers L.C. (2004). Oxidative damage to methyl-CpG sequences inhibits the binding of the methyl-CpG binding domain (MBD) of methyl-CpG binding protein 2 (MeCP2). Nucleic Acids Research, 32, 4100-4108. 
Van den Donk M., Pellis L., Crott J.W., Van Engeland M., Friederich P., Nagengast F.M., Van Bergeijk J.D., Boer S.Y., Mason J.B., Kok F.J., Keijer J., Kampman E. (2007a). Folic acid and vitamin $B_{12}$ supplementation does not favorably influence uracil incorporation and promoter methylation in rectal mucosa DNA of subjects with previous colorectal adenomas. American Society for Nutrition, 137, 2114-2120.

Van den Donk M., Van Engeland M., Pellis L., Witteman B.J., Kok F.J., Kampman E. (2007b). Dietary folate intake in combination with MTHFR C677T genotype and promoter methylation of tumor suppressor and DNA repair genes in sporadic colorectal adenomas. Cancer Epidemiology Biomarkers and Prevention, 16 (2), 327-333.

Van der Heijden G.W., Ramos L., Baart E.B., Van den Berg I.M., Derijck A.A., Van der Vlag J., Martini E., De Boer P. (2008). Sperm-derived histones contribute to zygotic chromatin in humans. BMC Developmental Biology, 8, 34.

Van Guelpen B., Hultdin J., Johansson I., Hallmans G., Stenling R., Riboli E., Winkvist A., Palmgvist R. (2006). Folate levels may protect against colorectal cancer. International Journal of Gastroenterology and Hepatology, 55 (10), 1461-1466.

Van Engeland M., Weijenberg M.P., Roemen G.M., Brink M., Bruine A.P., Goldbohm R.A., Brandt P.A., Baylin S.B., Goeij A.F.P.M., Herman J.G. (2003). Effects of dietary folate and alcohol intake on promoter methylation in sporadic colorectal cancer: The Netherlands cohort study on diet and cancer. Cancer Research, 63 (12), 3133-3137.

Van Rooij I., Ocke M.C., Straatman H., Zielhuis G.A., Merkus H., SteegersTheunissen R. (2004). Periconceptional folate intake by supplement and food reduces the risk of nonsyndromic cleft lip with or without cleft palate. Preventive Medicine, 39 (4), 689-694.

Vilarino-Guell C., Smith A.G., Dubrova Y.E. (2003). Germline mutation induction at mouse repeat DNA loci by chemical mutagens, Mutation Research, 526, 63-73.

Vineis P., Chuang S.C., Vaissiere T., Cuenin C., Ricceri F., Johansson M., Ueland Per., Brennan P., Herceg Z. (2011). DNA methylation changes associated with cancer risk factors and blood levels of vitamin metabolites in a prospective study. Epigenetics, 6 (2), 195-201.

Voorrips L.E., Goldbohm R.A., Brants H.A.M., Poppel G.A.F.C., Sturmans F., Hermus R.J.J., Brandt P.A. (2000). A prospective cohort study on antioxidant and folate intake and male lung cancer risk. Cancer Epidemiology Biomarkers and Prevention, 9, 357-365. 
Voutounou M., Glen C., Dubrova Y.E. (2012). The effects of methyl-donor deficiency on mutation induction and transgenerational instability in mice. Mutation Research, 734, 1-4.

Wagner C. (1995). Biochemical role of folate in cellular metabolism, in: L.B. Bailey (Ed.), Folate in Health and Disease. Marcel Dekker, 23-42.

Wainfan E., Poirier L.A. (1992). Methyl groups in carcinogenesis: effects on DNA methylation and gene expression. Cancer Research, 52, 2071-2077.

Wallock, L.M., Tamura T., Mayr C.A., Johnston K.E., Ames B.N., Jacob R.A. (2001). Low seminal plasma folate concentrations are associated with low sperm density and count in male smokers and nonsmokers. Fertility and Sterility, 75, 252-259.

Wakefield L., Boukouvala S., Sim E. (2010). Characterisation of CpG methylation in the upstream control region of mouse Nat2: Evidence for a gene-environment interaction in the polymorphic gene implicated in folate metabolism. Gene, 452 (1), 16-21.

Waterland R.A. (2006). Epigenetic mechanisms and gastrointestinal development. The Journal of Paediatrics, 149, 137-142.

Watts G.S., Futscher B.W., Holtan N., DeGeest K., Domann F.E., Rose S.L. (2008). DNA methylation changes in ovarian cancer are cumulative with disease progression and identify tumour stage. BMC Medical Genomics, 1, 47.

Weber M., Davies J.J., Wittig D., Oakeley E.J., Haase M., Lam W.L., Schubeler D. (2005). Chromosome-wide and promoter-specific analyses identify sites of differential DNA methylation in normal and transformed human cells. Nature Genetics, 37, 853-862.

Weber M., Hellmann I., Stadler M.B., Ramos L., Paabo S., Rebhan M., Schübeler D. (2007). Distribution, silencing potential and evolutionary impact of promoter DNA methylation in the human genome. Nature Genetics, 39, 457-466.

Weitzman S.A., Turk P.W., Milkowski D.H., Kozlowski K. (1994). Free radical adducts induce alterations in DNA cytosine methylation. Proceedings of the National Academy of Sciences U.S.A., 91, 1261-1264.

Weitzmann M.N., Woodford K.J., Usdini K. (1998). The mouse Ms6-hm hypervariable minisatellite forms a hairpin and two unusual tetraplexes. Journal of Biology and Chemistry, 273, 30742-30749.

Widschwendter M., Jiang G., Woods C., Muller H.M., Fiegl H., Goebel G., Marth C., Muller-Holzner E., Zeimet A.G., Laird P.W., Ehrlich M. (2004). DNA hypomethylation and ovarian cancer biology. Cancer Research, 64, 44724480. 
Wiemels J.L., Smith R.N., Taylor G.M., Eden O.B., Alexander F.E., Greaves M.F. (2001). Methylenetetrahydrofolate reductase (MTHFR) polymorphisms and risk of molecularly defined subtypes of childhood acute leukemia. Proceedings of the National Academy of Science USA, 98, 4004-4009.

Wilkinson L.S., Davies W., Isles A.R. (2007). Genomic imprinting effects on brain development and function. Nature, 8, 832-843.

Willett W.C., Trichopoulos D. (1996). Nutrition and cancer: A summary of the evidence. Cancer Causes and Control, 7, 178-180.

Williams C.L., Meck W.H., Heyer D., Loy R. (1998). Hypertrophy of basal forebrain neurons and enhanced visuospatial memory in perinatally choinesupplemented rats. Brain Research, 794, 225-238.

Williams R.W. (2000). In: Mapping genes that modulate brain development: a quantitative genetic approach. In: Mouse brain development. Goffinet A.F., Rakic P. (eds). Springer -Verlag, New York, pp 21-49.

Wilson J.W., Enns C.W., Goldman J.D., Tippett K.S., Mickle S.J., Cleveland L.E., Chahil P.S. (1997). Data tables: Combined results from USDA's 1994 and 1995 continuing survey of food intakes by individuals and 1994 and 1995 diet and health knowledge survey. U.S. Department of Agriculture, 1, 1-108.

Wilson A.S., Power B.E., Molloy P.L. (2007). DNA hypomethylation and human diseases. Biochemical and Biophysical Acta: Reviews on Cancer, 1775 (1), 138-162.

Wolffe A.P., Jones P.L., Wade P.A. (1999). DNA demethylation. Proceedings of the National Academy of Science U.S.A., 96, 5894-5896.

Wright, W.E., Shay, J.W. (2000). Telomere dynamics in cancer progression and prevention: Fundamental differences in human and mouse telomere biology. Nature Medicine, 6, 849-851.

Wu M.Y. Kuo C.S., Lin C.Y., Lu C.L., Syu-Huang R.F. (2009). Lymphocytic mitochondrial DNA deletions, biochemical folate status and hepatocellular carcinoma susceptibility in a case-control study. British Journal of Nutrition, 102 (5), 715-721.

Xing J., Kang L., Jiang Y. (2011). Effect of dietary betaine supplementation on lipogenesis gene expression and CpG methylation of lipoprotein lipase gene in broilers. Molecular Biology Reports, 38 (3), 1975-1981.

Yamada Y., Jackson-Grusby L., Linhart H., Meissner A., Eden A., Lin H., Jaenisch R. (2005). Opposing effects of DNA hypomethylation on intestinal and liver carcinogenesis. Proceedings of the National Academy of Sciences USA, 102, 13580-13585. 
Yang K., Kurihara N., Fan K., Newmark H., Rigas B., Bancroft L., Corner G., Livote E., Lesser M., Edelmann W., Velcich A., Lipkin M., Augenlicht L. (2008). Dietary induction of colonic tumors in a mouse model of sporadic colon cancer. Cancer Research, 68, 7803-7810.

Yamane K., Toumazou C., Tsukada Y., Erdjument-Bromage H., Tempst P. (2006). JHDM2A, a JmjC-containing H3K9 demethylase, facilitates transcription activation by androgen receptor. Cell, 125, 483-495.

Yauk CL., Dubrova Y.E., Grant G.R., Jeffreys A.J. (2002). A novel single molecule analysis of spontaneous and radiation-induced mutation at a mouse tandem repeat locus. Mutation Research, 500, 147-156.

Yauk C.L. (2004). Advanves in the application of germline tandem repeat instability for in-situ monitoring. Mutation Research, 556 (2), 169-82.

Yauk C.L. Berndt M.L., Williams A., Douglas G.R. (2004). Comprehensive comparison of six microarray technologies. Nucleic Acids Research, 32 (15), 1-7.

Yauk, C.L., Polyzos A., Rowan-Carroll A., Kortubash I., Williams A., Kovalchuk O. (2008). Tandem repeat mutation, global DNA methylation, and regulation of DNA methyltransferases in cultured mouse embryonic fibroblast cells chronically exposed to chemicals with different modes of action. Environmental Molecular Mutagenesis, 49, 26-35.

Yeh A., Wei M., Golub S.B., Yamashiro D.J., Murty V.V., Tycko B. (2003). Chromosome arm 16q in Wilms tumours: Unbalanced chromosomal translocations, loss of heterozygosity, and assessment of the CTCF gene. Genes Chromosomes and Cancer, 35, 156-163.

Yoder J.A., Walsh C.P., Bestor T.H. (1997). Cytosine methylation and the ecology of intragenomic parasites. Trends Genetics, 13, 335-340.

Young S.S., Eskenazi B., Marchetti F.M., Block G., Wyrobek A.J. (2008). The association of folate, zinc and antioxidant intake with sperm aneuploidy in healthy non-smoking men. Human Reproduction, 23,1014-1022.

Zalenskaya I.A., Bradbury E.M., Zalensky A.O. (2000). Chromatin structure of telomere domain in human sperm. Biochemical and Biophysical Research Communications, 279, 213-218.

Zeisel S.H. (2006). Choline: Critical role during fetal development and dietary requirements in adults. Annual Review of Nutrition, 26, 229-250.

Zhang S.M., Willett W.C., Selhub J., Hunter D.J., Giovannucci E.L., Holmes M.D., Colditz G.A., Hankinson S.E. (2003). Plasma folate, vitamin $B_{6}$, vitamin $\mathrm{B}_{12}$, homocysteine, and risk of breast cancer. Journal of the National Cancer Institute, 95 (5), 373-380. 
Zhao C.Q., Young M.R., Diwan B.A., Coogan T.P., Waalkes M.P. (1997). Association of arsenic-induced malignant transformation with DNA hypomethylation and aberrant gene expression. Proceedings of the National Academy of Sciences U.S.A., 94, 10907-10912.

Zheng N., Monckton D. G., Wilson G., Hagemeister F., Chakraborty R., Connor T. H., Siciliano M. J., Meistrich M. L. (2000). Frequency of minisatellite repeat number changes at the MS205 locus in human sperm before and after cancer chemotherapy. Environmental and Molecular Mutagenesis, 36, 134-145.

Zhu H., Geiman T.M., Xi S., Jiang Q., Schmidtmann A., Chen T., Li E., Muegge K. (2006). Lsh is involved in de novo methylation of DNA. EMBO Journal, 25, 335-345.

Zhu J.K. (2009). Active DNA demethylation mediated by DNA glycosylases. Annual Reviews Genetics, 43, 143-166. 